PNNL-17639

\title{
Deposition Velocities of Newtonian and Non-Newtonian Slurries in Pipelines
}

$\begin{array}{ll}\text { AP Poloski } & \text { F Nigl } \\ \text { HE Adkins } & \text { MJ Minette } \\ \text { J Abrefah } & \text { JJ Toth } \\ \text { AM Casella } & \text { JM Tingey } \\ \text { RE Hohimer } & \text { ST Yokuda }\end{array}$

March 2009 


\title{
DISCLAIMER
}

This report was prepared as an account of work sponsored by an agency of the United States Government. Neither the United States Government nor any agency thereof, nor Battelle Memorial Institute, nor any of their employees, makes any warranty, express or implied, or assumes any legal liability or responsibility for the accuracy, completeness, or usefulness of any information, apparatus, product, or process disclosed, or represents that its use would not infringe privately owned rights. Reference herein to any specific commercial product, process, or service by trade name, trademark, manufacturer, or otherwise does not necessarily constitute or imply its endorsement, recommendation, or favoring by the United States Government or any agency thereof, or Battelle Memorial Institute. The views and opinions of authors expressed herein do not necessarily state or reflect those of the United States Government or any agency thereof.

\author{
PACIFIC NORTHWEST NATIONAL LABORATORY \\ operated by \\ BATTELLE \\ for the \\ UNITED STATES DEPARTMENT OF ENERGY \\ under Contract DE-AC05-76RL01830 \\ Printed in the United States of America \\ Available to DOE and DOE contractors from the \\ Office of Scientific and Technical Information, \\ P.O. Box 62, Oak Ridge, TN 37831-0062; \\ ph: (865) 576-8401 \\ fax: (865) 576-5728 \\ email: reports@adonis.osti.gov
}

Available to the public from the National Technical Information Service, U.S. Department of Commerce, 5285 Port Royal Rd., Springfield, VA 22161 ph: (800) 553-6847

fax: (703) 605-6900

email: orders $a$ ntis.fedworld.gov

online ordering: http://www.ntis.gov/ordering.htm

This document printed on recycled paper. 
PNNL-17639

WTP-RPT-175 Rev. 0

\section{Deposition Velocities of Newtonian and Non-Newtonian Slurries in Pipelines}

$\begin{array}{ll}\text { AP Poloski } & \text { F Nigl } \\ \text { HE Adkins } & \text { MJ Minette } \\ \text { J Abrefah } & \text { JJ Toth } \\ \text { AM Casella } & \text { JM Tingey } \\ \text { RE Hohimer } & \text { ST Yokuda }\end{array}$

Test Scoping Statement(s): $\quad$ SCN 023

Test Specification:

24590-WTP-TSP-RT-07-005, Rev. 0

Test Plan:

TP-RPP-WTP-493, Rev. 0

Test Exception(s): None

March 2009

Prepared for

the U.S. Department of Energy

under Contract DE-AC05-76RL01830

Pacific Northwest National Laboratory

Richland, Washington 99352 


\section{Completeness of Testing}

This report describes the results of work and testing specified by Test Specification 24590 WTP TSP-RT-07-005, Rev. 0, and Test Plan TP-RPP-WTP-493, Rev. 0. The work and any associated testing followed the quality assurance requirements outlined in the Test Specification, and Test Plan. The descriptions provided in this test report are an accurate account of both the conduct of the work and the data collected. Test plan results are reported. Also reported are any unusual or anomalous occurrences that are different from expected results. The test results and this report have been reviewed and verified.

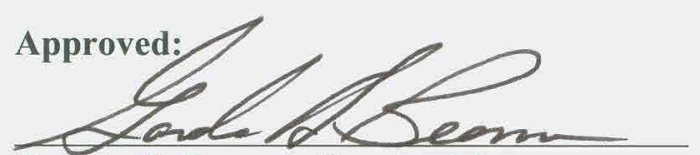

Gordon H. Beeman, Program Manager RPP-WTP Support Program

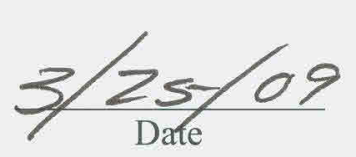




\section{Executive Summary}

The External Flowsheet Review Team (EFRT) expressed concern about the potential for Waste Treatment and Immobilization Plant (WTP) pipe plugging. Per the review's executive summary, "Piping that transports slurries will plug unless it is properly designed to minimize this risk. This design approach has not been followed consistently, which will lead to frequent shutdowns due to line plugging." To evaluate the potential for plugging, critical-velocity tests were performed on several physical simulants to determine if the design approach is conservative. Critical velocity is defined as the point where particles begin to deposit to form a moving bed of particles on the bottom of a straight horizontal pipe during slurry transport operations. The critical velocity depends on the physical properties of the particles, fluid, and system geometry.

This report gives the results from critical-velocity testing and provides an indication of slurry stability as a function of fluid rheological properties and transport conditions that are typical of what the plant will see. The experimental results are compared to the WTP design guide on slurry-transport velocity in an effort to confirm minimum waste-velocity and flushing-velocity requirements as established by calculations and critical-velocity correlations in the design guide. The major findings of this testing are as follows:

Experimental results indicate that for Newtonian fluids, the design guide is conservative. The design guide is based on the Oroskar and Turian (1980) correlation, a traditional industry-derived equation that focuses on particles larger than $100 \mu \mathrm{m}$ in size. Slurry viscosity has a greater effect on particles with a larger surface area to mass ratio, i.e. smaller particles. The increased viscous forces on small particles result in a smaller critical velocities. Since the Hanford slurry particles generally have large surface area to mass ratios, the reliance on such equations in the 24590-WTP-GPG-M-0058, Rev 0 design guide (Hall 2006) is conservative. Additionally, the use of the $95 \%$ percentile particle size as an input to this equation is conservative. The design guide specifies the use of the $d_{95}$ density, this term is ambiguous and needs clarification in the design guide. Nonetheless, this value is interpreted to mean the density of the $d_{95}$ particle. This density value is irrelevant for critical velocity calculations. Often this value is unknown, and Equation 1 of the 24590-WTP-GPG-M-0058, Rev 0 design guide (Hall 2006) will be used for design purposes. This equation calculates an average or composite density of all solids in the slurry. However, test results indicate that the use of an average particle density as an input to the equation is not conservative. Particle density has a large influence on the overall critical-velocity result returned by the correlation. The viscosity correlation used in the WTP design guide has been shown to be inaccurate for Hanford waste feed materials. Additionally, the recommendation of a 30\% minimum margin on predictions accounts for the majority of data scatter in the equations. However, to account for engineering margin, an additional increase in the design margin is recommended for application to the WTP. Lastly, the original Oroskar and Turian (1980) equation has been modified with a parameter that accounts for the homogeneous and heterogeneous fractions of the slurry. As explained below, this modification leads to a non-conservative result in some instances. In other instances, the conservatism of the unmodified Oroskar and Turian (1980) correlation for small particles is usually large enough to overcome these non-conservative inputs.

The use of the Thomas (1979) correlation in the design guide if applied to non-Newtonian fluids or for very small \& dense particles is not conservative. The design guide returns a non-conservative value from the Thomas (1979) correlation rather than from the conservative Oroskar and Turian (1980) correlation in cases where $100 \%$ of the particles are smaller than 74 $\mu \mathrm{m}$ or in the presence of yield stress forces. The measured data in this report show that the Thomas (1979) correlation predictions often fall below measured experimental values. The measured deposition velocity values in this report are often greater than the limiting critical velocity predictions. The limiting critical velocity predictions also often fall in unstable flow regions on so-called "stability maps." For this reason, the limiting critical velocity maynot be conservative.

A non-Newtonian deposition-velocity design guide is recommended to be developed for the WTP. Since the WTP expects to process large quantities of non-Newtonian slurries, the existing design guide is recommended to be modified to address such non-Newtonian slurries. A central finding of this testing is that particle deposition in non-Newtonian slurries usually occurs near the flow velocity required to reach turbulent flow. This velocity increases with slurry rheological properties (yield stress and infinite shear viscosity) because of viscous forces that dampen the formation of turbulent eddies. Since the turbulent eddies necessary for particle transport are not present, the particles will settle when the velocity falls below this threshold, called the transitional deposition boundary. This deposition mechanism should be reflected through new correlations in a recommended design guide for non-Newtonian fluids. 



\section{Acknowledgments}

The authors would like to acknowledge the assistance of the following staff members in supporting entire project including construction of the flow loop, conducting research, and obtaining the needed data.

$\begin{array}{lll}\text { JM Alzheimer } & \text { PJ MacFarlan } & \text { CC Duncan } \\ \text { BE Butcher } & \text { WR Park } & \text { CE Guzman-Leong } \\ \text { WH Combs } & \text { JH Sachs } & \text { NK Karri } \\ \text { RC Daniel } & \text { GL Smith } & \text { M Luna } \\ \text { AD Guzman } & \text { L Zhong } & \text { KL Peterson } \\ \text { CC Kovalchick } & \text { SK Bapanapalli } & \text { DS Sklarew }\end{array}$





\section{Testing Summary}

The U.S. Department of Energy (DOE) Office of River Protection's Waste Treatment and Immobilization Plant (WTP) will process and treat radioactive waste in the underground storage tanks at the Hanford Site. Piping, pumps, and mixing vessels will transport, store, and mix the high-level waste (HLW) slurries in the WTP.

The WTP pipe plugging issue, as stated by the External Flowsheet Review Team (EFRT) Executive Summary, is as follows: "Piping that transports slurries will plug unless it is properly designed to minimize this risk. This design approach has not been followed consistently, which will lead to frequent shutdowns due to line plugging." Additional details relating to the EFRT summary are provided in a supplemental background document. ${ }^{2}$ The WTP Project is implementing a strategy to address the above EFRT issue identified as "Issue M1—Plugging in Process Piping."

The tests described herein address the need to perform critical-velocity tests on physical simulants. Critical velocity is defined as the point where a moving bed of particles begins to deposit on the bottom of a straight horizontal pipe during slurry-transport operations. Deposition testing of slurries containing particles with well-characterized size and density suspended in fluids with known rheological properties was performed. The test results provide information to evaluate the conditions that can lead to plugging in pipeline transport operations.

The simulant test particles ranged in density from 2.5 to $8 \mathrm{~g} / \mathrm{cc}$ while the nominal particle size ranged from 10 to $100 \mu \mathrm{m}$. Seventeen tests were conducted with these test particles suspended in three carrier fluids with target Bingham plastic yield-stress values of 0,3 , and $6 \mathrm{~Pa}$.

An experimental flow loop was constructed of 3 -inch schedule 40 stainless steel piping with a mixing tank, slurry pump, and instrumentation for determining flow rate and pressure gradient. At the beginning of a test, the slurry flow velocity was nominally set to $8 \mathrm{ft} / \mathrm{sec}$. The flow was then incrementally decreased and a steady-state pressure gradient was obtained at each flow condition. A rise in pressure gradient as the flow rate drops indicates that the pipe cross-sectional area is filled with moving sediment. This point is referred to as the "critical velocity." The laminar-to-turbulent transition velocity is referred to as the "transition velocity."

To clear the sediment bed from the system, a flush system modeled after the WTP design (as of August 2007) was installed and tested. This system consists of a pressure vessel where an initial charge of water is placed. The pressure is then increased to a target value, nominally 100 to $110 \mathrm{psig}$. A valve is opened, and the high-pressure water flush removes deposited slurry particles from the pipe loop.

Results from this testing provide an indication of slurry stability as a function of fluid rheological properties and transport conditions. Moreover, the experimental results are compared to the WTP design

\footnotetext{
${ }^{1}$ WTP Project Doc. No. CCN 132846 "Comprehensive Review of the Hanford Waste Treatment Plant Flowsheet and Throughput-Assessment Conducted by an Independent Team of External Experts," March 2006. Available online at: http://www.hanford.gov/orp/uploadfiles/Final_ERT\%20Review\%20of\%20EAC.pdf. Accessed 06/13/08.

${ }^{2}$ WTP Project Doc. No. CCN 132847 "Background Information and Interim Reports for the Comprehensive Review of the Hanford Waste Treatment Plant Flowsheet and Throughput-Assessment Conducted by an Independent Team of External Experts,” March 2006.
} 
guide $^{3}$ in an effort to confirm minimum waste-velocity and flushing-velocity requirements as established by calculations and critical line-velocity correlations in the design guide. The design guide is said to be "conservative" if it predicts critical line velocities higher than those observed experimentally at conditions relevant to WTP operation.

The 24590-WTP-GPG-M-0058, Rev 0 design guide (Hall 2006) consists of nine steps. Table S.1 summarizes the results of testing by describing each of the nine steps and then commenting on whether the guide's predictions were conservative compared to the experimental results. Additional observations are also included.

Table S.1. Nine Steps of the 24590-WTP-GPG-M-0058, Rev 0 design guide (Hall 2006)

\begin{tabular}{|c|c|}
\hline $\begin{array}{l}\text { Design-Guide } \\
\text { Step Number }\end{array}$ & $\begin{array}{l}\text { 24590-WTP-GPG-M-0058, Rev } 0 \text { Design Guide Description (top) } \\
\text { Experimental Results and Observations(bottom) }\end{array}$ \\
\hline \multirow[t]{2}{*}{1} & $\begin{array}{l}\text { The design guide specifies the use of the } d_{95} \text { particle diameter for line transfers and the } d_{99} \text { particle } \\
\text { diameter for line flushes. }\end{array}$ \\
\hline & Experimental results indicate that this specification is conservative. \\
\hline \multirow[t]{2}{*}{2} & $\begin{array}{l}\text { The design guide specifies the use of the } d_{95} \text { density, this term is ambiguous and needs clarification } \\
\text { in the design guide. Nonetheless, this value is interpreted to mean the density of the } d_{95} \text { particle. } \\
\text { This density value is irrelevant for critical velocity calculations. Often this value is unknown, and } \\
\text { Equation } 1 \text { of the design guide (Hall 2006) will be used for design purposes. This equation } \\
\text { calculates an average or composite density of all solids in the slurry. }\end{array}$ \\
\hline & $\begin{array}{l}\text { Experimental results indicate that the WTP design-guide protocol of using average solids density } \\
\text { in the calculations is not conservative. Discrete particles with densities much greater than the } \\
\text { average solids density can settle and cause pipeline plugging under WTP process conditions. }\end{array}$ \\
\hline \multirow[t]{4}{*}{3} & $\begin{array}{l}\text { For Newtonian slurries, the design guide specifies the determination of the fraction of particles that } \\
\text { are above } 74 \mu \mathrm{m} \text {. These particles are considered to be the heterogeneous or settling fraction of the } \\
\text { slurry. For non-Newtonian fluid with a yield stress, Darby (2000) suggests that yield stress can } \\
\text { support the particles as they are being transported. }\end{array}$ \\
\hline & $\begin{array}{l}\text { Experimental results indicate that small, dense particles settle as superficial velocity decreases } \\
\text { toward the transition velocity. }\end{array}$ \\
\hline & $\begin{array}{l}\text { The WTP design-guide protocol of using a 200-mesh }(74-\mu m) \text { cut-off for homogeneous carrier } \\
\text { fluid in calculations is not conservative. Particles below } 74 \mu \mathrm{m} \text { settle and cause pipeline } \\
\text { plugging under WTP process conditions. }\end{array}$ \\
\hline & $\begin{array}{l}\text { The WTP design-guide protocol of using a Bingham yield-stress cut-off for homogeneous } \\
\text { carrier fluid in calculations is not conservative. Particles normally suspended by yield stress } \\
\text { in a non-moving system settle and cause pipeline plugging under flowing conditions. }\end{array}$ \\
\hline
\end{tabular}

\footnotetext{
${ }^{3}$ WTP Project Doc. No. 24590-WTP-GPG-M-0058, Rev 0, Minimum Flow Velocity for Slurry Lines, November 27, 2006.
} 
Table S.1. (contd)

\begin{tabular}{|c|c|}
\hline $\begin{array}{l}\text { Design-Guide } \\
\text { Step Number }\end{array}$ & $\begin{array}{l}\text { 24590-WTP-GPG-M-0058, Rev } 0 \text { Design Guide Description (top) } \\
\text { Experimental Results and Observations(bottom) }\end{array}$ \\
\hline \multirow[t]{2}{*}{4} & $\begin{array}{l}\text { The design guide specifies calculation of the density of the slurry resulting from the combination of } \\
\text { interstitial liquid and the homogeneous particles. }\end{array}$ \\
\hline & $\begin{array}{l}\text { One should note that a mathematical error exists in the equation to calculate the homogenous } \\
\text { carrier fluid density in Step } 4 \text { of the } 24590-W T P-G P G-M-0058, \text { Rev } 0 \text { design guide (Hall 2006). } \\
\text { The homogeneous portion solids volume fraction should be used in the denominator of Equation } \\
\text { (8) of the } 24590-W T P-G P G-M-0058 \text {, Rev } 0 \text { design guide (Hall 2006) rather than the } \\
\text { heterogeneous portion solids-volume fraction. This substitution is needed for conservation of mass } \\
\text { in the slurry system. For the systems considered in this report, this correction only results in about } \\
\text { a } 5 \% \text { increase in the calculated critical velocities. }\end{array}$ \\
\hline \multirow[t]{2}{*}{5} & $\begin{array}{l}\text { The WTP design guide protocol of using the RPP- } 9805 \text { correlation (Jewett et al. 2002) that uses } \\
\text { solids loading to calculate apparent viscosity of the Hanford tank farm feed is not accurate. }\end{array}$ \\
\hline & $\begin{array}{l}\text { The WTP design-guide protocol of using the RPP-9805 correlation (Jewett et al. 2002) relating } \\
\text { solids loading to apparent viscosity of the Hanford tank farm feed is not accurate because: } \\
\text { o Many physiochemical variables affect slurry rheology, including pH, dissolved salt content, } \\
\text { particle size, solid-phase morphology, and system surface chemistry. } \\
\text { o The slurry physical and rheological properties of each process stream need to be considered } \\
\text { rather than primarily the feed stream. }\end{array}$ \\
\hline \multirow[t]{2}{*}{6} & $\begin{array}{l}\text { The design guide specifies that the hindered settling factor is typically } 0.95 \text { for Hanford tank sludge } \\
\text { applications. }\end{array}$ \\
\hline & Experimental results indicate that this specification is satisfactory. \\
\hline \multirow[t]{2}{*}{7} & $\begin{array}{l}\text { The variables defined in Steps } 1 \text { to } 6 \text { are used as inputs to the Oroskar and Turian (1980) critical- } \\
\text { velocity correlation. }\end{array}$ \\
\hline & $\begin{array}{l}\text { Experimental results indicate that for Newtonian fluids, the design guide (based on the Oroskar } \\
\text { and Turian (1980) correlation) is conservative. This traditional industry-derived correlation is } \\
\text { based on behavior for particles larger than } 100 \text { pm in size, and increased viscous forces on smaller } \\
\text { particles with a larger surface area-to-mass ratio results in decreased actual critical velocities. } \\
\text { However, the Oroskar and Turian (1980) equation has been modified from the original form with a } \\
\text { parameter that accounts for the homogeneous and heterogeneous fractions of the slurry. As } \\
\text { explained in the following step, this modification leads to a non-conservative result in some } \\
\text { instances. }\end{array}$ \\
\hline \multirow[t]{2}{*}{8} & $\begin{array}{l}\text { The variables defined in Steps } 1 \text { to } 6 \text { are used as inputs to the Thomas (1979) critical-velocity } \\
\text { correlation. In the } 24590 \text {-WTP-GPG-M-0058, Rev } 0 \text { design guide (Hall 2006), this is referred to as } \\
\text { the "limiting critical velocity." }\end{array}$ \\
\hline & $\begin{array}{l}\text { In cases where } 100 \% \text { of the particles are smaller than } 74 \mu \text { m or for non-Newtonian fluids, where } \\
\text { particles are considered to be homogeneous because of yield stress forces suspending the particles } \\
\text { (Darby 2000), the design guide sets the homogeneous fraction of the slurry to } 100 \% \text {. In such } \\
\text { cases, the predicted critical velocity based on step } 7 \text { is reduced to zero and the "limiting critical } \\
\text { velocity" becomes the bounding equation. The measured deposition velocity values in this report } \\
\text { are often greater than the limiting critical velocity predictions. The limiting critical velocity } \\
\text { predictions also often fall in unstable flow regions on so-called "stability maps." For this reason, } \\
\text { the limiting critical velocity may not be conservative. }\end{array}$ \\
\hline
\end{tabular}


Table S.1. (contd)

\begin{tabular}{|c|c|}
\hline $\begin{array}{l}\text { Design-Guide } \\
\text { Step Number }\end{array}$ & $\begin{array}{l}\text { 24590-WTP-GPG-M-0058, Rev } 0 \text { Design Guide Description (top) } \\
\text { Experimental Results and Observations(bottom) }\end{array}$ \\
\hline \multirow[t]{2}{*}{9} & $\begin{array}{l}\text { A minimum design margin of } 30 \% \text { will be applied to the largest critical velocity between } V_{O T} \text { and } \\
V_{T} \text { determined in Steps } 7 \text { and } 8 \text {. The resulting value is the recommended design pipeline superficial } \\
\text { velocity. }\end{array}$ \\
\hline & $\begin{array}{l}\text { Turian et al. (1987) present statistical data on several alternative empirical forms of the Oroskar } \\
\text { and Turian (1980) equation and report that } 8 \% \text { of the literature data have a deviation above } 50 \% \text {, } \\
\text { and } 38 \% \text { of the data have a deviation above } 20 \% \text {. These empirical equations have a maximum } \\
\text { deviation on the order of } 120 \% \text {. In this context, the } 24590 \text {-WTP-GPG-M-0058, Rev } 0 \text { design guide } \\
\text { (Hall 2006) recommendation of a } 30 \% \text { minimum margin on predictions would account for the } \\
\text { majority of data scatter in the equations. However, to account for engineering margin, an } \\
\text { additional increase in the design margin is recommended for application to the WTP. }\end{array}$ \\
\hline
\end{tabular}

Since the 24590-WTP-GPG-M-0058, Rev 0 design guide (Hall 2006) is limited to Newtonian fluids and the WTP expects to process large quantities of non-Newtonian slurries, the existing design guide should be modified to address deposition velocity calculations for non-Newtonian slurries. Specifically, a design guide for critical velocity of both Newtonian and non-Newtonian fluids is recommended be developed and consist of at least three criteria: 1) the critical deposition boundary, 2) the transitional deposition boundary, and 3) the laminar deposition boundary. These boundaries define the transitions between several slurry-transport flow regimes. Four of the major regimes are shown schematically in Figure S.1.

The three boundaries between these four regimes are strong functions of slurry rheological properties. By plotting yield stress on the abscissa and flow velocity on the ordinate, a "stability map" showing these stability regimes can be obtained. An example stability map is shown in Figure S.2. Point W on the abscissa (i.e., rheological properties equivalent to water) represents the Newtonian critical deposition velocity for turbulent flow. This point can be predicted by the Oroskar and Turian (1980) and Shook et al. (2002) equations. As the non-Newtonian character of the slurry increases, adding yield stress, the increased apparent viscosity of the slurry adds drag on the particles and reduces the flow velocity needed to suspend the particles in turbulent flow. The boundary between the stable turbulent and unstable turbulent flow regimes is referred to as the critical deposition boundary and is depicted by path WX (shown in blue).

However, the flow velocity required to reach turbulent flow increases with slurry rheological properties because viscous forces dampen the formation of turbulent eddies. At point X, the flow becomes dominated by viscous forces rather than turbulent eddies. Since the turbulent eddies necessary for particle transport are not present, the particles will settle when flow velocity crosses this boundary, called the transitional-deposition boundary. Along path XY, the stable turbulent and unstable laminar flow regimes are defined.

As the yield stress continues to increase the required pressure gradient for flow increases. At point $Y$, the required pressure gradient is adequate to push the particles through the pipeline even along the pipeline wall. Often yield stress forces are large enough to suspend the particles in the stagnant core region of a non-Newtonian flow. Along path YZ, the rheological properties continue to increase, which lowers the flow velocity required to achieve the pressure gradient required to push the particles through the pipeline. 
Hence, for particles in slurries to remain suspended, pipelines must be operated in Regime A or Regime D. At the WTP design-basis velocity of 4 to $6 \mathrm{ft} / \mathrm{sec}$, however, under some rheological conditions expected to be present in the WTP process streams, flow falls within Regimes B \& C, and particle deposition is likely to occur. This report presents the equations that define where these boundaries fall with respect to particle and rheological properties. This stability-map concept might be furthermore considered as a basis for a revision to the WTP design guide for non-Newtonian fluids.

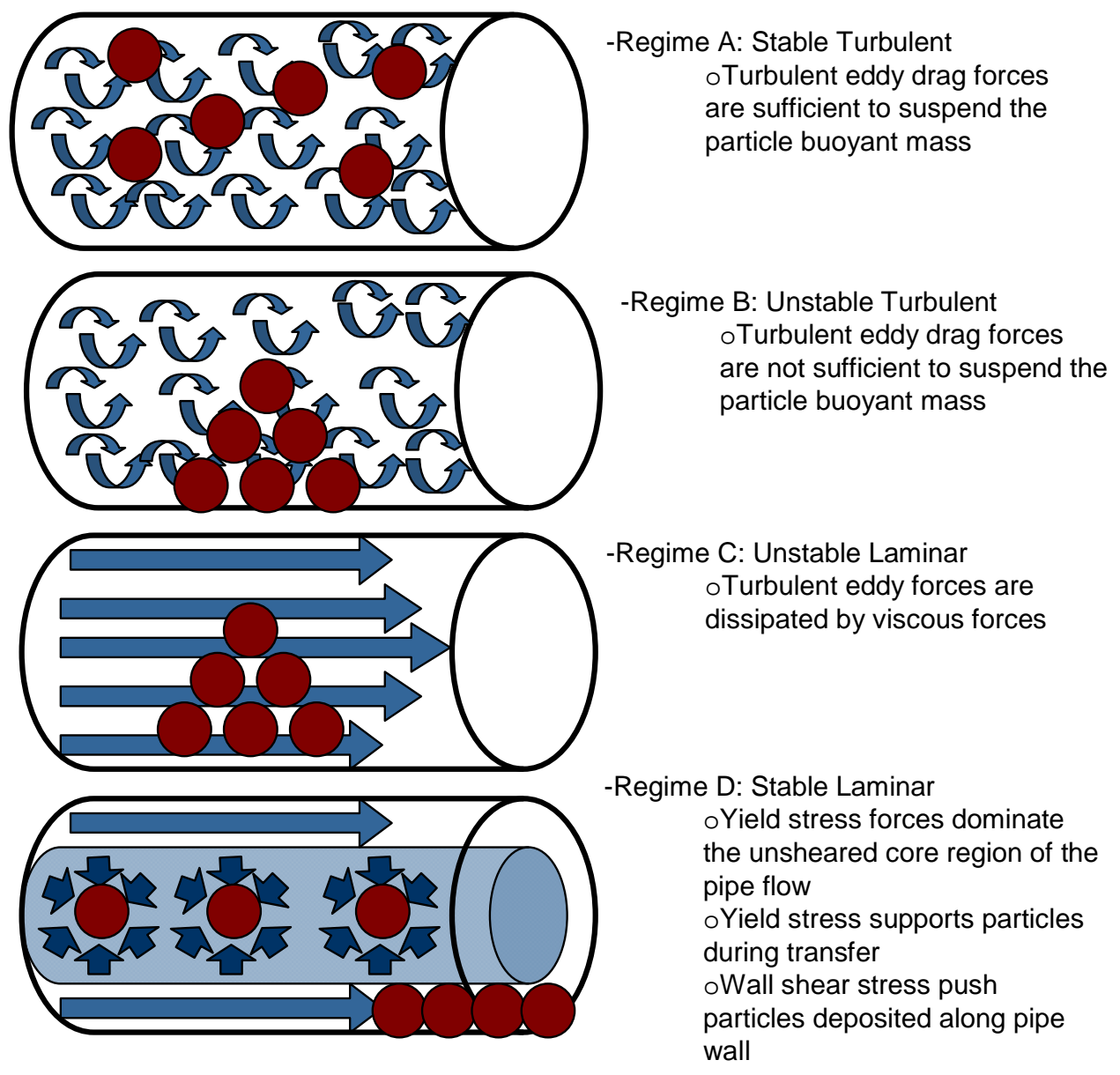

Figure S.1. Four Slurry-Transport Flow Regimes 


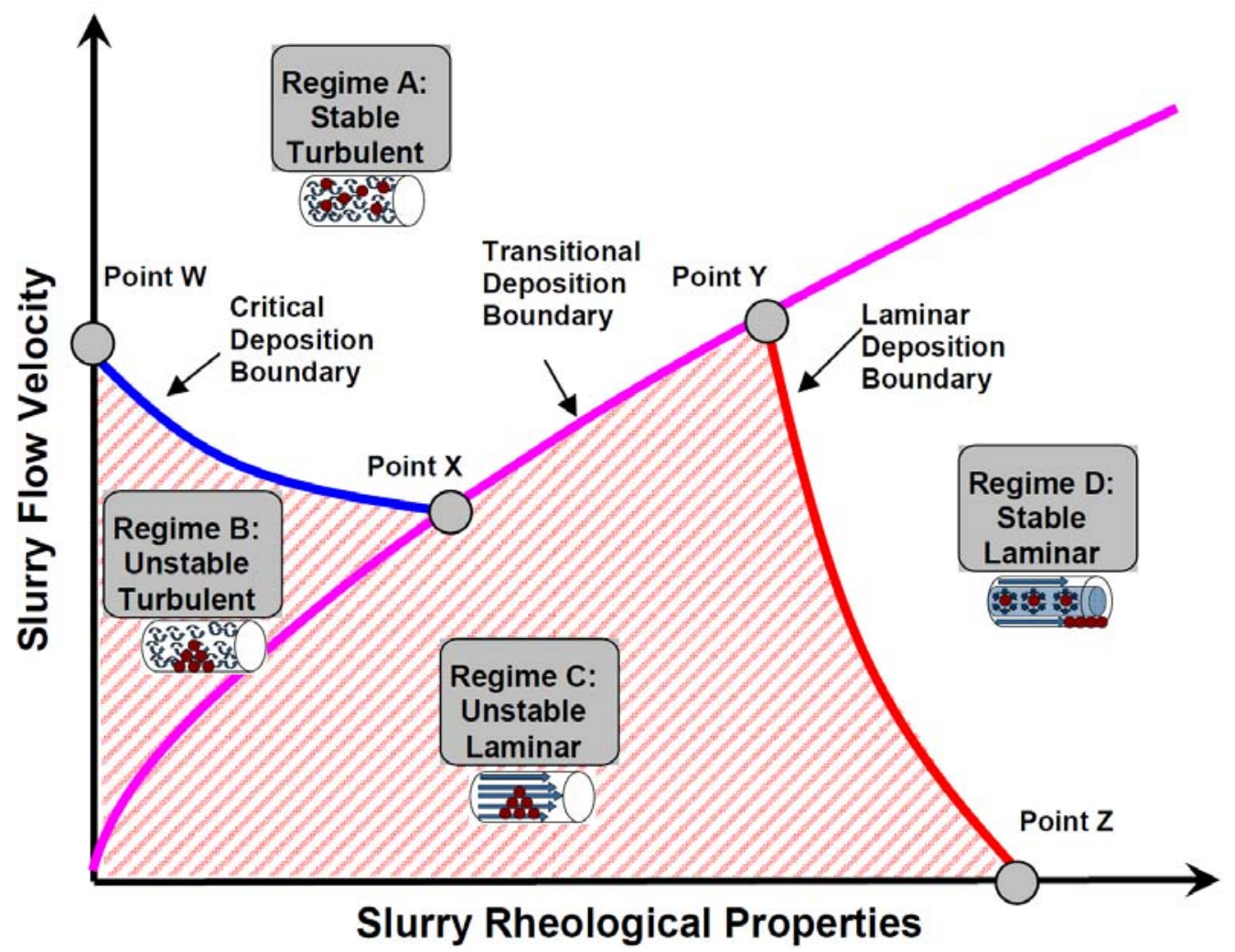

Figure S.2. Example of a Stability Map

Lastly, the flush system effectively removed sediment beds from the piping with the following caveats:

- The pneumatic flush system must be opened slowly to erode the sediment bed from the top down. If the pneumatic flush system is opened quickly, the sediment bed is simply pushed to the nearest corner, and a granular plug develops and completely fills the pipeline cross-sectional area. During testing a granular plug was slowly forced through the system by cycling pressure to the line on and off. In the transparent section, the velocity of this granular plug was observed to be on the order of inches per second. It took several minutes to remove this granular bed. Cycling the valves open and closed during these situations appeared to accelerate recovery operations. Operation in this mode resulted in water hammer as the flow stopped and started. Interestingly, the most difficulties occurred with the low-rheology tests. Higher rheology likely offers a higher apparent viscosity on the particles in the sediment bed with an associated increase in shear stress that promotes plug removal.

- A minimum flush-to-line volume ratio of 1.5 to 1.7 appears to be slightly low. Even though the density of the flush solution exiting the flow loop approaches the flush water density in this volume ratio range, a small amount of particles was still observed in the transparent section. A minimum flush-to-line volume ratio of 2 would likely produce only a trace amount of particles in the piping.

- The 24590-WTP-GPG-M-0058, Rev 0 design guide (Hall 2006) requirements on flush volume and flush velocity were difficult to achieve manually. Achieving these requirements would likely involve a closed-loop feedback system consisting of a flow meter, a level indicator, and an automatic control valve. 


\section{S.1 Test Objectives}

The objectives are specified in Test Specification 24590-WTP-TSP-RT-07-005, Rev. 0 (BNI 2007) and test results are discussed in Table S.2.

Table S.2. Test Objectives and Results

\begin{tabular}{lll}
\hline \multicolumn{1}{c}{ Test Objective } & $\begin{array}{c}\text { Objective Met? } \\
\text { (Yes/No) }\end{array}$ & \multicolumn{1}{c}{ Results } \\
\hline $\begin{array}{l}\text { Confirm minimum waste-velocity and } \\
\text { flushing-velocity requirements as } \\
\text { established by calculations and } \\
\text { critical line-velocity correlations in } \\
\text { the design guide. }\end{array}$ & No & $\begin{array}{l}\text { Testing was completed but did not confirm the } \\
\text { hypothesis as stated in the test objective. Calculations } \\
\text { and critical line velocity correlations in the design } \\
\text { guide are conservative for Newtonian systems but } \\
\text { may not be conservative if applied to non-Newtonian } \\
\text { systems. }\end{array}$ \\
\hline
\end{tabular}

(a) WTP Project Doc. No. 24590-WTP-GPG-M-0058, Rev 0, Minimum Flow Velocity for Slurry Lines, November 27, 2006

\section{S.2 Test Exceptions}

No test exception was applied to this investigation.

\section{S.3 Results and Performance Against Success Criteria}

The success criteria are specified in test specification 24590-WTP-TSP-RT-07-005, Rev. 0 (BNI 2007), and test results are discussed in Table S.3.

Table S.3. Success Criteria and Results

\begin{tabular}{cl}
\hline Success Criteria & \multicolumn{1}{c}{ Results } \\
\hline & $\begin{array}{l}\text { For Newtonian slurries this success criterion was met. Critical velocity for } \\
\text { several slurry systems was measured experimentally. For the Newtonian cases, } \\
\text { the design-guide predictions were made with a margin that exceeded the } \\
\text { measured values. }\end{array}$ \\
$\begin{array}{c}\text { Verify that solids do not settle at } \\
\text { the design basis velocity. }\end{array}$ & $\begin{array}{l}\text { For non-Newtonian slurries this success criterion was not met. For the non- } \\
\text { Newtonian cases, no design guide for critical velocity exists. However, the } \\
\text { design guide for a Bingham plastic pressure drop was consulted, and a } \\
\text { homogeneity criterion from this guide was used to perform an assessment. The } \\
\text { results indicate that despite passing this homogeneity criterion, slurries settle } \\
\text { and act heterogeneously. }\end{array}$ \\
$\begin{array}{c}\text { Determine the velocity at which } \\
\text { solids settle to document the } \\
\text { design margin. }\end{array}$ & $\begin{array}{l}\text { Success criterion was met. In the case of small particles (less than 74 } \mu \text { m) in } \\
\text { Newtonian fluids, the actual design-guide margin was as low as 7\%. For all of } \\
\text { the coarse particles tested, the design guide margin was as high as 42\%. }\end{array}$ \\
$\begin{array}{c}\text { Demonstrate the adequacy of the } \\
\text { design basis to avoid plugging } \\
\text { due to particle settling in piping. }\end{array}$ & $\begin{array}{l}\text { Success criterion was not met. Test results indicate that settling is expected to } \\
\text { occur for non-Newtonian fluids under the current design-guide implementation. } \\
\text { However, the flushing system appears to be adequate to remove the sediment } \\
\text { beds tested. }\end{array}$ \\
\hline
\end{tabular}




\section{S.4 Quality Requirements}

The Quality Assurance Program at Pacific Northwest National Laboratory (PNNL) is based on requirements defined in U.S. Department of Energy (DOE) Order 414.1C, Quality Assurance, and 10 CFR 830, Energy/Nuclear Safety Management, Subpart A-Quality Assurance Requirements (a.k.a. the Quality Rule). PNNL has chosen to implement the requirements of DOE Order 414.1C and 10 CFR 830, Subpart A by integrating them into the Laboratory's management systems and daily operating processes. The procedures necessary to implement the requirements are documented through PNNL's StandardsBased Management System.

PNNL implements the RPP-WTP quality requirements by performing work in accordance with the River Protection Project-Waste Treatment Plant Support Program (RPP-WTP) Quality Assurance Plan (RPP-WTP-QA-001, QAP). Work was performed to the quality requirements of NQA-1-1989 Part I, Basic and Supplementary Requirements, NQA-2a-1990, Part 2.7, and DOE/RW-0333P, Rev 13, Quality Assurance Requirements and Descriptions $(Q A R D)$. These quality requirements are implemented through the River Protection Project-Waste Treatment Plant Support Program (RPP-WTP) Quality Assurance Manual (RPP-WTP-QA-003, QAM).

\section{S.5 Test Conditions}

Test conditions were controlled with administrative hold points. Several hold points are indentified in the Test Condition section of Test Specification 24590-WTP-TSP-RT-07-005, Rev. 0 (BNI 2007). These hold points were translated into PNNL Test Plan TP-RPP-WTP-493, Rev. 0. The status of each of the hold points is summarized in Table S.4:

Table S.4. Status of Project Hold Points

\begin{tabular}{llll}
\hline \multicolumn{1}{c}{ Test Plan } & \multicolumn{1}{c}{ Hold Point } & Approved On & \multicolumn{1}{c}{$\begin{array}{c}\text { WTP Project } \\
\text { Document Number }\end{array}$} \\
\hline $\begin{array}{l}\text { TP-RPP-WTP-493, } \\
\text { Rev. 0 }\end{array}$ & $\begin{array}{l}\text { \#1 a. BNI approve Test Plan. } \\
\text { b. BNI approve test simulants. }\end{array}$ & $\begin{array}{l}\text { a. } 6 / 19 / 07 \\
\text { b. } 11 / 9 / 07\end{array}$ & $\begin{array}{l}\text { a.(Signed Test Plan) } \\
\text { b. CCN 163048 }\end{array}$ \\
& $\begin{array}{l}\text { \#2 a. BNI approve part number identification } \\
\text { (P\&ID)/drawing before fabrication. } \\
\quad \text { b. BNI approve test matrix. }\end{array}$ & a. 6/21/07 & a. CCN 153217 \\
& $\begin{array}{l}\text { \#3 BNI define flush tank operating pressures } \\
\text { and an acceptable solids residue after flushing } \\
\text { tests. }\end{array}$ & b. 12/17/07 & b. CCN 163054 \\
\end{tabular}

\section{S.6 Simulant Use}

Physical simulants were used in this testing. As discussed in the Test Conditions section, hold point \#1 allowed BNI to review and accept the test simulants before testing. A detailed description of simulant development goals and properties are presented in this report. 


\section{S.7 Discrepancies and Follow-on Tests}

- Follow-on Test Recommendation \#1-To further increase the confidence in the results presented in this report, a series of complex simulant tests are recommended to be performed. The complex simulant should be designed to mimic the complex mixture of particle sizes and densities expected to be processed in the WTP. This can be accomplished though the use of a simulant that possesses matching chemical, physical, and rheological properties of Hanford tank waste.

- Follow-on Test Recommendation \#2-The boundary for operating slurry-transport systems in stable laminar flow is not well defined in the literature. Additional tests with physical simulants at increased rheological properties would allow for the development of a laminar flow stability criterion. This would allow the operation of the WTP under laminar flow conditions with moderate-to-high rheology (15 to $30 \mathrm{~Pa}$ Bingham yield stress) at the current design velocities of 4 to $6 \mathrm{ft} / \mathrm{sec}$. 



\section{Acronyms and Abbreviations}

BNI

CFR

DLVO

DOE

DOE-EM

EFRT

ERT

GFC

$\mathrm{HHH}$

HHL

HHM

HHX

HLH

HLL

HLM

HLW

HLX

HTWOS

IEP

ITS

LAW

LHH

LHL

LHM

LHX
Bechtel National, Inc.

Code of Federal Regulations

Derjaquin, Landau, Verwey, and Overbeek (theory)

U.S. Department of Energy

DOE's Office of Environmental Management

External Flowsheet Review Team

electrical resistance tomography

glass-former chemical

high density, high particle size, high rheological property simulant; i.e. $100 \mu \mathrm{m}$ nominal stainless steel in $6 \mathrm{~Pa}$ yield stress kaolin/water slurry

high density, high particle size, low rheological property simulant; i.e. $100 \mu \mathrm{m}$ nominal stainless steel in water

high density, high particle size, medium rheological property simulant; i.e. $100 \mu \mathrm{m}$ nominal stainless steel in $3 \mathrm{~Pa}$ yield stress kaolin/water slurry

high density, high particle size simulant; i.e. $100 \mu \mathrm{m}$ nominal stainless steel in all carrier fluids

high density, low particle size, high rheological property simulant; i.e. $10 \mu \mathrm{m}$ nominal stainless steel in $6 \mathrm{~Pa}$ yield stress kaolin/water slurry

high density, low particle size, low rheological property simulant; i.e. $10 \mu \mathrm{m}$ nominal stainless steel in water

high density, low particle size, medium rheological property simulant; i.e. $10 \mu \mathrm{m}$

nominal stainless steel in $3 \mathrm{~Pa}$ yield stress kaolin/water slurry

high level waste

high density, low particle size simulant; i.e. $10 \mu \mathrm{m}$ nominal stainless steel in all carrier fluids

Hanford Tank Waste Operation Simulator

iso-electric point

Industrial Tomography Systems

low-activity waste

low density, high particle size, high rheological property simulant; i.e. $100 \mu \mathrm{m}$ nominal glass beads in $6 \mathrm{~Pa}$ yield stress kaolin/water slurry

low density, high particle size, low rheological property simulant; i.e. $100 \mu \mathrm{m}$ nominal glass beads in water

low density, high particle size, medium rheological property simulant; i.e. $100 \mu \mathrm{m}$ nominal glass beads in $3 \mathrm{~Pa}$ yield stress kaolin/water slurry

low density, high particle size simulant; i.e. $100 \mu \mathrm{m}$ nominal glass beads in all carrier fluids 
LLH low density, low particle size, high rheological property simulant; i.e. $10 \mu \mathrm{m}$ nominal glass beads in $6 \mathrm{~Pa}$ yield stress kaolin/water slurry

LLL low density, low particle size, low rheological property simulant; i.e. $10 \mu \mathrm{m}$ nominal glass beads in water

LLM low density, low particle size, medium rheological property simulant; i.e. $10 \mu \mathrm{m}$ nominal glass beads in $3 \mathrm{~Pa}$ yield stress kaolin/water slurry

LLX low density, low particle size simulant; i.e. $10 \mu \mathrm{m}$ nominal glass beads in all carrier fluids

$\mathrm{MMH}$

medium density, medium particle size, high rheological property simulant; i.e. $50 \mu \mathrm{m}$ nominal alumina in $6 \mathrm{~Pa}$ yield stress kaolin/water slurry

MML medium density, medium particle size, low rheological property simulant; i.e. $50 \mu \mathrm{m}$ nominal alumina in water

MMM

medium density, medium particle size, medium rheological property simulant; i.e. 50 $\mu \mathrm{m}$ nominal alumina in $3 \mathrm{~Pa}$ yield stress kaolin/water slurry

MMX

medium density, medium particle size simulant; i.e. $50 \mu \mathrm{m}$ nominal alumina in all carrier fluids

NIST

National Institute of Standards and Technology

P\&ID

Piping and Instrumentation Diagram

PDD

PNNL

particle-density distribution

PSD

Pacific Northwest National Laboratory

PVC

particle-size distribution

QA

polyvinyl chloride

QAM

quality assurance

QAP

Quality Assurance Manual

QARD

Quality Assurance Plan

RPP

Quality Assurance Requirements and Descriptions

WTP

River Protection Project

WTPSP

Hanford Waste Treatment and Immobilization Plant

WTP Support Project 


\section{Nomenclature}

\begin{tabular}{|c|c|}
\hline$A_{H}$ & the Hamaker constant \\
\hline$A r$ & $\begin{array}{l}\text { the Archimedes Number can be defined as the ratio of gravitational forces on } \\
\text { the particle corrected for buoyancy to viscous forces on the particle }\end{array}$ \\
\hline$A r_{\infty}$ & $\begin{array}{l}\text { the Archimedes Number defined for non-Newtonian fluids using an infinite } \\
\text { shear viscosity }\end{array}$ \\
\hline $\mathrm{b}$ & Hershel-Bulkley power law exponent \\
\hline $\mathrm{Ca}$ & the Casson Number \\
\hline$C_{c}$ & the coarse particle volume fraction (i.e., particles exceeding $74 \mu \mathrm{m}$ ) \\
\hline$C_{D}$ & the drag coefficient \\
\hline$C_{f}$ & fine fraction of solids is determined by $C_{f}=\eta_{h o m o} \cdot C_{s}$ \\
\hline$d$ & particle diameter \\
\hline$D$ & pipe inner diameter \\
\hline$D_{0}$ & equilibrium-state separation distance between the particles \\
\hline$d_{50}$ & $50 \mathrm{vol} \%$ of particles are smaller than this particle diameter \\
\hline$d_{95}$ & $95 \mathrm{vol} \%$ of particles are smaller than this particle diameter \\
\hline$d_{99}$ & 99 vol $\%$ of particles are smaller than this particle diameter \\
\hline$D_{y}$ & separation distance between the particles at the point of yielding \\
\hline$f$ & Fanning friction factor \\
\hline$F$ & force \\
\hline $\mathrm{Fr}$ & $\begin{array}{l}\text { the Froude Number can be defined as the ratio of inertial forces in the fluid } \\
\text { flow to the gravitational forces the particle must overcome to stay suspended } \\
\text { in the flow }\end{array}$ \\
\hline$g$ & gravitational acceleration \\
\hline$h$ & the Planck constant \\
\hline $\mathrm{He}$ & the Hedstrom number \\
\hline$k$ & Boltzmann's constant \\
\hline $\mathrm{k}$ & Herschel-Bulkley consistency coefficient \\
\hline$l_{E}$ & eddy length scale \\
\hline $\mathrm{L}$ & length of pipe \\
\hline$m$ & the power law consistency coefficient \\
\hline$n$ & the power law exponent \\
\hline$n$ & the refractive index \\
\hline
\end{tabular}




\begin{tabular}{|c|c|}
\hline$r$ & particle radius \\
\hline $\operatorname{Re}$ & Reynolds number \\
\hline$R e_{t}$ & Reynolds number at the laminar-to-turbulent flow transition velocity \\
\hline$S$ & ratio of the coarse solid density to carrier fluid density, $\rho_{S} / \rho_{f}$ \\
\hline$s_{\text {turb }}$ & a constant used in Equation (A.37) \\
\hline$T$ & temperature \\
\hline$u^{*}$ & the friction velocity, $\sqrt{\frac{\tau_{w}}{\rho_{f}}}$ \\
\hline$V$ & fluid bulk velocity \\
\hline$v^{\prime}$ & eddy-velocity fluctuations \\
\hline$V$ & velocity \\
\hline$V_{1}$ & $\begin{array}{l}\text { velocity at or above which the bed in the lower half of the pipe is stationary. } \\
\text { In the upper half of the pipe, some solids may move by saltation or } \\
\text { suspension. }\end{array}$ \\
\hline$V_{2}$ & $\begin{array}{l}\text { velocity at or above which the mixture flows as an asymmetric mixture with } \\
\text { the coarser particles forming a moving bed. }\end{array}$ \\
\hline$V_{3,}, V_{c}$ & $\begin{array}{l}\text { velocity at or above which all particles move as an asymmetric suspension } \\
\text { and below which the solids start to settle and form a moving bed }\end{array}$ \\
\hline$V_{4}$ & velocity at or above which all solids move as a symmetric suspension \\
\hline$v_{e}$ & the frequency of electron cloud oscillations \\
\hline$V_{N}$ & the equivalent Newtonian fluid velocity \\
\hline$V_{O T}$ & the Oroskar and Turian (1980) critical velocity \\
\hline$V_{t}$ & the laminar-to-turbulent flow transition velocity \\
\hline$V_{T}$ & the Thomas (1979) critical velocity \\
\hline$Y$ & $\begin{array}{l}\text { the yield-stress stability parameter; If greater than } 0.2 \text {, the particle is } \\
\text { supported by yield stress and considered homogeneous or non-settling. }\end{array}$ \\
\hline$\alpha$ & $\begin{array}{l}\text { the ratio of the average surficial particle shear stress, } \tau_{p} \text {, to the wall shear } \\
\text { stress, } \tau_{w}\end{array}$ \\
\hline$\dot{\gamma}$ & shear rate \\
\hline$\Delta \mathrm{P}$ & Pressure drop \\
\hline$\varepsilon$ & dielectric constant of the medium \\
\hline$\varepsilon_{0}$ & permittivity of a vacuum \\
\hline$\varepsilon_{r r}$ & roughness of the pipe \\
\hline$\zeta$ & the zeta potential \\
\hline
\end{tabular}




\begin{tabular}{|c|c|}
\hline$\eta$ & the Newtonian viscosity \\
\hline$\eta_{\text {homo }}$ & $\begin{array}{l}\text { fraction of particles less than } 74 \mu \mathrm{m} \text { that are considered to be homogeneous or } \\
\text { non-settling }\end{array}$ \\
\hline$\kappa$ & inverse Debye length. \\
\hline$\mu_{\text {eff }}$ & the fluid effective viscosity \\
\hline$\mu_{B}$ & the Bingham plastic infinite shear viscosity \\
\hline$\mu_{C}$ & the Casson infinite shear viscosity \\
\hline$\mu_{f}$ & carrier fluid dynamic viscosity \\
\hline$\mu_{W}$ & the carrier fluid dynamic viscosity (assumed to be water) \\
\hline$\xi$ & $\begin{array}{l}\text { the dimensionless ratio of fluid yield stress to shear stress at the pipe wall, } \\
\tau_{y} / \tau_{w}\end{array}$ \\
\hline$\rho_{f}$ & fluid or slurry density \\
\hline$\rho_{m}$ & the slurry mixture density \\
\hline$\tau$ & shear stress \\
\hline$\tau_{B}$ & the Bingham yield stress \\
\hline$\tau_{C}$ & the yield-stress fit to the Casson fluid model \\
\hline$\tau_{H}$ & the Herschel-Bulkley yield stress \\
\hline$\tau_{p}$ & average surficial particle shear stress \\
\hline$\tau_{\mathrm{w}}$ & the wall shear stress \\
\hline$\tau_{y}$ & the yield stress of the fluid \\
\hline$\Phi_{\varepsilon}$ & electrostatic interaction potential \\
\hline$\Phi$ & interaction potential \\
\hline$\phi$ & the solids volume fraction \\
\hline$\Phi_{v d W}$ & van der Waals interaction potential \\
\hline$\chi$ & hindered settling factor. \\
\hline$\psi_{S}$ & surface charge potential \\
\hline
\end{tabular}





\section{Contents}

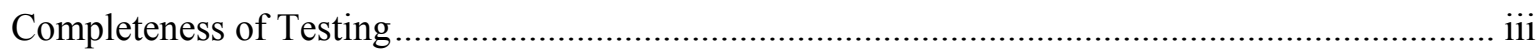

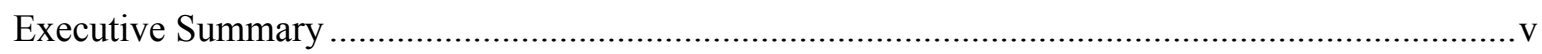

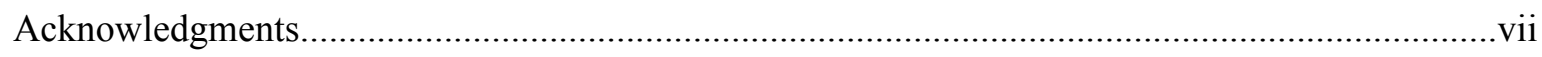

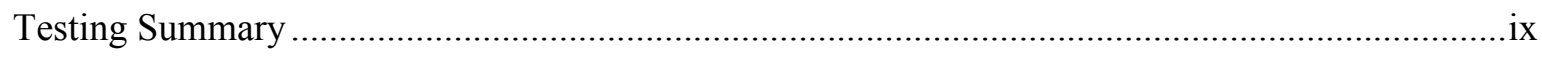

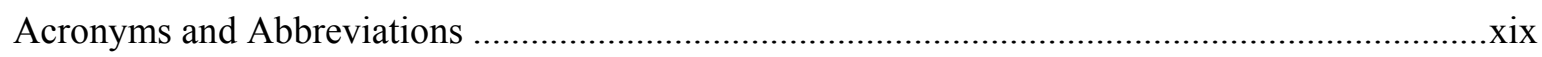

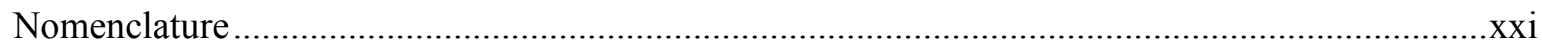

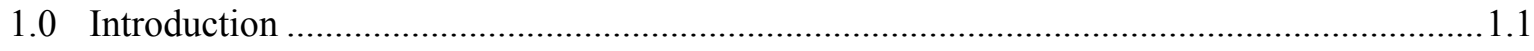

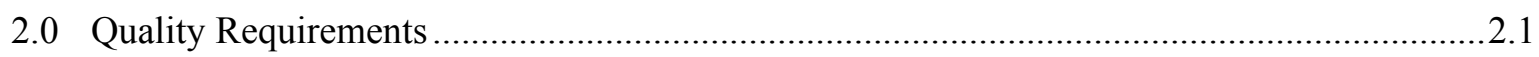

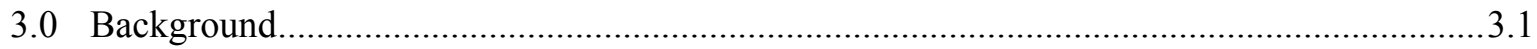

3.1 WTP Design Guide for Critical Velocity …............................................................... 3.3

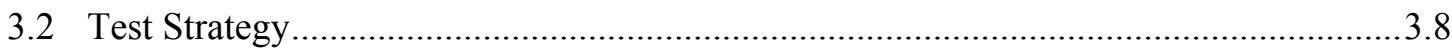

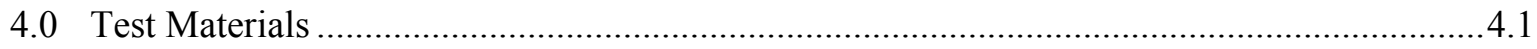

4.1 Expected Range of Hanford Particle Density ............................................................ 4.1

4.2 Expected Range of Hanford Particle Size ............................................................................ 4.2

4.3 Expected Range of Hanford Sludge Rheology ............................................................... 4.3

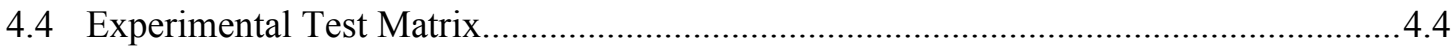

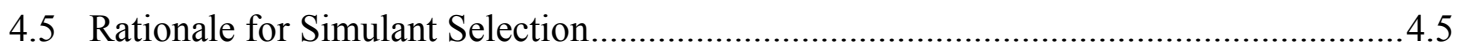

4.6 Simulant Composition and Physical Properties ............................................................. 4.6

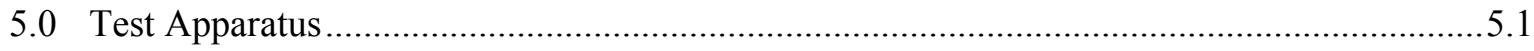

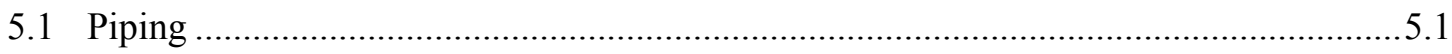

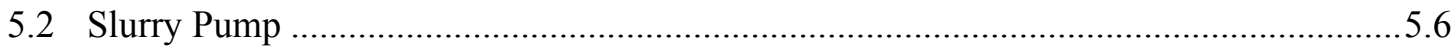

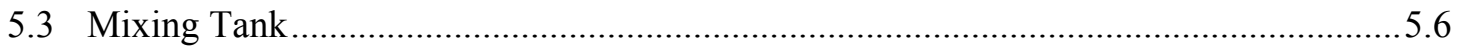

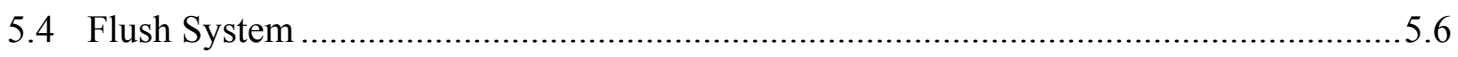

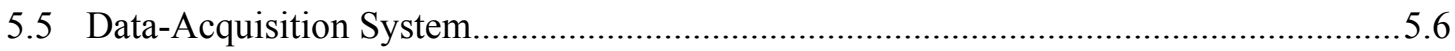

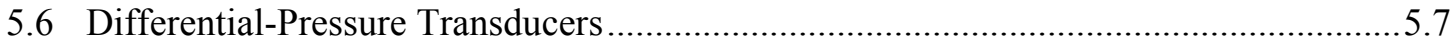

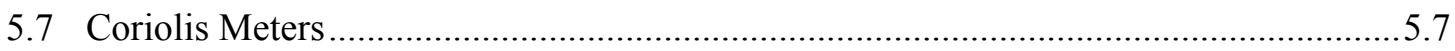

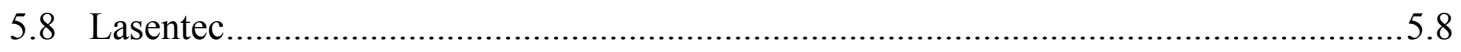

5.9 Electrical Resistance Tomography ........................................................................ 5.10

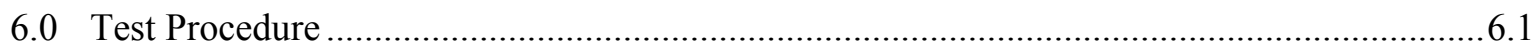

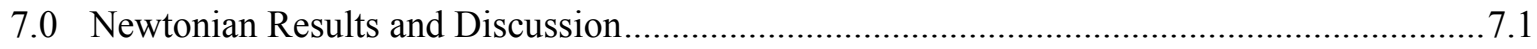

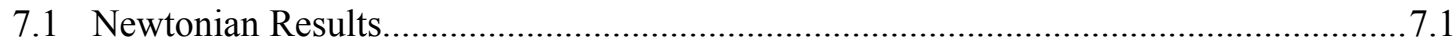

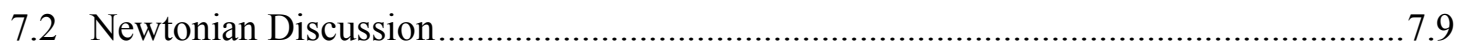

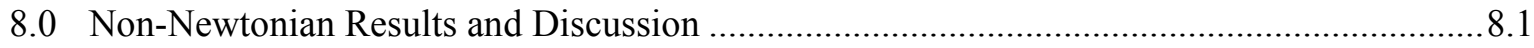

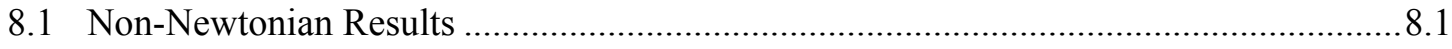

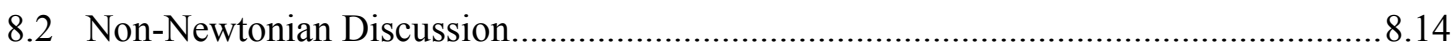


9.0 Recommendations for a non-Newtonian Design Guide ..................................................9.1

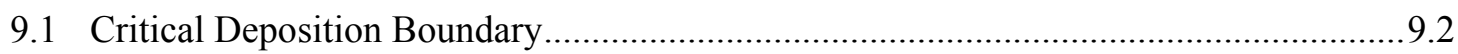

9.1.1 Extension to Non-Newtonian Systems ……...................................................... 9.4

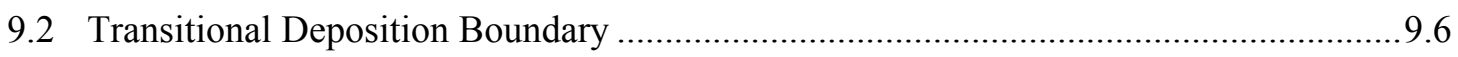

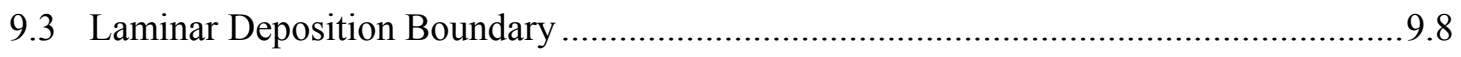

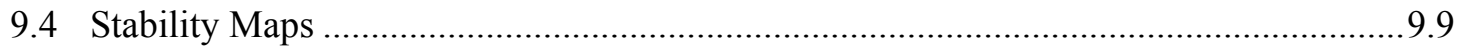

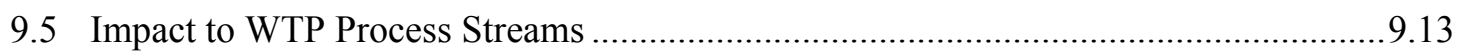

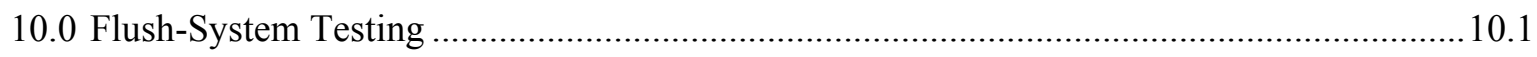

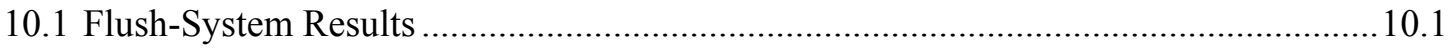

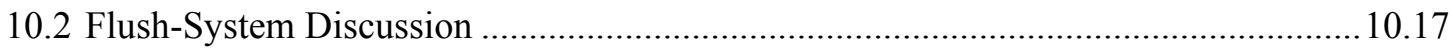

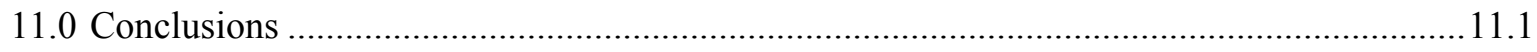

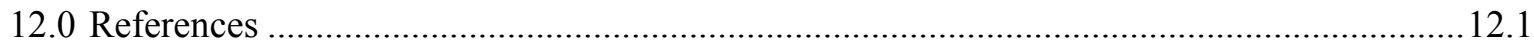

Appendix A Background Information and Correlations for Slurry Transport Calculations ............ A.1

Appendix B Lasentec Results .............................................................................................. B. 1

Appendix C Pressure Drop Versus Velocity for Water ............................................................. C.1 


\section{Figures}

Figure 3.1. Effects of Particle Concentration and Interparticle Attraction on Fluid Rheology (adapted from Brown and Heywood (1991)

Figure 3.2. Photograph of Flow Loop System with Major Equipment Identified; Arrows and Numbers Indicate Normal Operation Flow Direction.

Figure 4.1. Top: Timeline Showing the Ratio of Volume of Slurry Particulate Compounds to the Volume of Aluminum Oxide as Gibbsite Prior to Leaching. Bottom: Timeline showing the ratio of volume of slurry particulate compounds to the volume of Aluminum Oxide as Gibbsite After Leaching. Note that this plot is unreviewed and is provided "for information only."

Figure 4.2. PSD of the Composite Hanford Tank Farm Slurries Reported in WTP-RPT-153, Rev. 0 (Wells et al. 2007); Distributions for minimal disturbance ("all md") and sonicated ("all sonic") waste states are given. Highlighted Regions Show the Binning Strategy Used for Simulant Design.

Figure 4.3. Maximum Measured Bingham Yield Stress for Various Hanford Tanks and Waste Types

Figure 4.4. Slurry Property Inputs for Significant Pipeline Transport Calculations.....

Figure 4.5. Micrograph of LL Coarse Particles (10- $\mu \mathrm{m}$ glass beads; Potters Industry Spheriglass $\circledR^{\circledR}$ A-glass 5000)

Figure 4.6. Micrograph of HL Coarse Particles (10- $\mu$ m stainless steel; AMETEK P316L 10 M)4.13

Figure 4.7. Micrograph of MM Coarse Particles (50- $\mu \mathrm{m}$ Alumina; Washington Mills Duralum® 220 grit).

Figure 4.8. Micrograph of HH Coarse Particles (100- $\mu \mathrm{m}$ stainless steel; AMETEK P316L $100+170)$

Figure 4.9. Micrograph of LH Coarse Particles (100- $\mu \mathrm{m}$ glass beads; Potters Industry

Spheriglass ${ }^{\circledR}$ A-glass 2227)

Figure 5.1. Schematic of Flow Loop System.....

Figure 5.2. Schematic of Flow Loop Piping System with Major Dimensions.

Figure 5.3. Photograph of Georgia Iron Works 2X3LCC Slurry Pump (source: www.giwindustries.com)

Figure 5.4. Schematic of Mixing Tank Internal Components.......................................................5.7

Figure 5.5. Schematic of Mixing Tank Internal Components.......................................................

Figure 5.6. Schematic of Lasentec ${ }^{\mathrm{TM}}$ D600S Probe (source: Lasentec ${ }^{\mathrm{TM}}$ Operation Manual) ........5.9

Figure 5.7. Schematic of Lasentec ${ }^{\mathrm{TM}}$ Measurement Concept (source: Lasentec ${ }^{\mathrm{TM}}$ Operation Manual)

Figure 5.8. Right - Schematic of ERT Pipeline Sensor Electrode Layout. (source: P2000 Operation Manual) Left - Installed PVC Transparent Pipe Section, ERT Pipeline Probe, and ERT Electronic Data-Acquisition System

Figure 6.1. Pressure Drop as a Function of Pipeline Superficial Velocity.....

Figure 7.1. Pressure Drop and Electrical Resistivity Tomograms for LLL Simulant (10- $\mu \mathrm{m}$ glass beads in water) as a Function of Pipeline Velocity. Note that ERT tomograms are not calibrated to NIST standards and are considered "for information only."..... 
Figure 7.2. Pressure Drop and Electrical Resistivity Tomograms for HLL Simulant (10- $\mu \mathrm{m}$ stainless steel in water) as a Function of Pipeline Velocity. Note that ERT tomograms are not calibrated to NIST standards and are considered "for information only.".

Figure 7.3. Pressure Drop and Electrical Resistivity Tomograms for MML Simulant (50- $\mu \mathrm{m}$ alumina in water) as a Function of Pipeline Velocity. Note that ERT tomograms are not calibrated to NIST standards and are considered "for information only."....

Figure 7.4. Pressure Drop and Electrical Resistivity Tomograms for LHL Simulant $(100-\mu \mathrm{m}$ glass beads in water) as a Function of Pipeline Velocity. Note that ERT tomograms are not calibrated to NIST standards and are considered "for information only.".....

Figure 7.5. Pressure Drop and Electrical Resistivity Tomograms for HHL Simulant (100- $\mu \mathrm{m}$ stainless steel in water) as a Function of Pipeline Velocity. Note that ERT tomograms are not calibrated to NIST standards and are considered "for information only.".

Figure 7.6. Plot that Accounts for Decreasing Fluid Density to Identify the Critical Velocity for the HHL Simulant (100- $\mu \mathrm{m}$ stainless steel in water).

Figure 7.7. Experimentally Measured and Corresponding 24590-WTP-GPG-M-0058, Rev 0 Design Guide (Hall 2006) Calculated Values for Critical Velocity of Newtonian systems .. 7.10

Figure 7.8. Experimentally Measured and Corresponding 24590-WTP-GPG-M-0058, Rev 0 Design Guide (Hall 2006) Calculated Values for Critical Velocity of Newtonian Systems .7.12

Figure 8.1. Pressure Drop and Electrical Resistivity Tomograms for LLM Simulant (10- $\mu \mathrm{m}$ glass beads in thin clay) as a Function of Pipeline Velocity. Note that ERT tomograms are not calibrated to NIST standards and are considered "for information only.".

Figure 8.2. Pressure Drop and Electrical Resistivity Tomograms for HLM Simulant (10- $\mu \mathrm{m}$ stainless steel in thin clay) as a Function of Pipeline Velocity. Note that ERT tomograms are not calibrated to NIST standards and are considered "for information only.".

Figure 8.3. Pressure Drop and Electrical Resistivity Tomograms for MMM Simulant, Run \#1 (50- $\mu \mathrm{m}$ alumina in thin clay) as a Function of Pipeline Velocity. Note that ERT tomograms are not calibrated to NIST standards and are considered "for information only."

Figure 8.4. Pressure Drop and Electrical Resistivity Tomograms for LHM Simulant (100- $\mu \mathrm{m}$ glass beads in thin clay) as a Function of Pipeline Velocity. Note that ERT tomograms are not calibrated to NIST standards and are considered "for information only.".

Figure 8.5. Pressure Drop and Electrical Resistivity Tomograms for HHM Simulant (100- $\mu \mathrm{m}$ stainless steel in thin clay) as a Function of Pipeline Velocity. Note that ERT tomograms are not calibrated to NIST standards and are considered "for information only.".

Figure 8.6. Pressure Drop and Electrical Resistivity Tomograms for LLH Simulant (10- $\mu$ m glass beads in thick clay) as a Function of Pipeline Velocity. Note that ERT tomograms are not calibrated to NIST standards and are considered "for information only."....

Figure 8.7. Pressure Drop and Electrical Resistivity Tomograms for HLH Simulant (10- $\mu \mathrm{m}$ stainless steel in thick clay) as a Function of Pipeline Velocity. Note that ERT tomograms are not calibrated to NIST standards and are considered "for information only.".....

Figure 8.8. Pressure Drop and Electrical Resistivity Tomograms for MMH Simulant (50- $\mu \mathrm{m}$ alumina in thick clay) as a Function of Pipeline Velocity. Note that ERT tomograms are not calibrated to NIST standards and are considered "for information only.".

Figure 8.9. Pressure Drop and Electrical Resistivity Tomograms for LHH Simulant (100- $\mu \mathrm{m}$ glass beads in thick clay) as a Function of Pipeline Velocity. Note that ERT tomograms are not calibrated to NIST standards and are considered "for information only.". 
Figure 8.10. Pressure Drop and Electrical Resistivity Tomograms for HHH Simulant $(100-\mu \mathrm{m}$ stainless steel in thick clay) as a Function of Pipeline Velocity. Note that ERT tomograms are not calibrated to NIST standards and are considered "for information only."

Figure 8.11. Yield-Stress Stability Calculations for non-Newtonian "Medium" and "High" Rheology Systems Using $d_{95}$ Particle Size and Solid Density

Figure 8.12. Experimentally Measured Values for Critical Velocity of non-Newtonian "Medium" and "High" Rheology Systems

Figure 8.13. Experimental and Corresponding 24590-WTP-GPG-M-0058, Rev 0 Design Guide (Hall 2006) Calculated Values of Particle Density for non-Newtonian "Medium" and "High" Rheology Systems.

Figure 8.14. Effective and Corresponding 24590-WTP-GPG-M-0058, Rev 0 Design Guide (Hall 2006) Calculated Values of Fluid Viscosity for non-Newtonian "Medium" and "High" Rheology Systems.

Figure 9.1. Graphical Interpretation of Four Major Slurry-Transport Flow Regimes

Figure 9.2. Graphical Illustration of a "Stability Map".

Figure 9.3. Dimensionless Deposition Froude Number Correlation for Archimedes Number Less than 80

Figure 9.4. Archimedes Number and Deposition Froude Number for both Newtonian and nonNewtonian Data

Figure 9.5. Transition and Deposition Froude Number for non-Newtonian Data

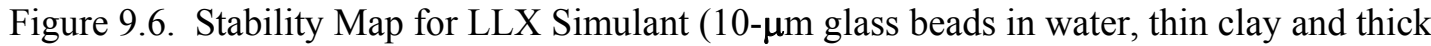
clay) as a Function of Pipeline Velocity and Yield Stress

Figure 9.7. Stability Map for HLX Simulant (10 $\mu \mathrm{m}$ stainless steel in water, thin clay and thick clay) as a Function of Pipeline Velocity and Yield Stress

Figure 9.8. Stability Map for MMX Simulant (50- $\mu \mathrm{m}$ alumina in water, thin clay and thick clay) as a Function of Pipeline Velocity and Yield Stress

Figure 9.9. Stability Map for LHX Simulant (100- $\mu \mathrm{m}$ glass beads in water, thin clay and thick clay) as a Function of Pipeline Velocity and Yield Stress

Figure 9.10. Stability Map for HHX Simulant (100- $\mu$ m stainless steel in water, thin clay and thick clay) as a Function of Pipeline Velocity and Yield Stress

Figure 9.11. Stability Map for $10-\mu \mathrm{m} \mathrm{PuO}_{2}$ Similar to the Inset Image in a Fluid with Rheological Parameters Resembling AZ-101 HLW Melter Feed. $\mathrm{PuO}_{2}$ image taken from SY-102 sample (Wells et al. 2007).

Figure 9.12. Stability Map for $150 \mu \mathrm{m}$ Olivine Similar to the Inset Image of LAW GFC Powder in a Fluid with Rheological Parameters Resembling AZ-102 LAW Melter Feed

Figure 9.13. Stability Map for $25 \mu \mathrm{m} \mathrm{Fe}_{2} \mathrm{O}_{3}$ Similar to the Inset Image of LAW GFC Powder in a Fluid with Rheological Parameters Resembling AZ-102 LAW Melter Feed

Figure 10.1. Flush Data for LLL Simulant (10- $\mu \mathrm{m}$ glass beads in water) as a Flush-to-Pipe Loop Volume Ratio Showing Three Complete Flushes...

Figure 10.2. Flush Data for HLL Simulant (10- $\mu \mathrm{m}$ stainless steel in water) as a Flush-to-Pipe Loop Volume Ratio Showing Three Complete Flushes

Figure 10.3. Flush Data for MML Simulant (50- $\mu \mathrm{m}$ alumina in water) as a Flush-to-Pipe Loop Volume Ratio Showing Two Complete Flushes. 
Figure 10.4. Flush Data for LHL Simulant (100- $\mu \mathrm{m}$ glass beads in water) as a Flush-to-Pipe Loop Volume Ratio Showing Three Complete Flushes

Figure 10.5. Flush Data for HHL Simulant (100- $\mu$ m stainless steel in water) as a Flush-to-Pipe Loop Volume Ratio Showing Four Complete Flushes

Figure 10.6. Flush Data for LLM Simulant (10- $\mu \mathrm{m}$ glass beads in thin clay) as a Flush-to-Pipe Loop Volume Ratio Showing Three Complete Flushes

Figure 10.7. Flush Data for HLM Simulant (10- $\mu \mathrm{m}$ stainless steel in thin clay) as a Flush-to-Pipe Loop Volume Ratio Showing Three Complete Flushes

Figure 10.8. Flush Data for MMM Simulant (50- $\mu \mathrm{m}$ alumina in thin clay) as a Flush-to-Pipe Loop Volume Ratio Showing Four Complete Flushes

Figure 10.9. Flush Data for LHM Simulant (100- $\mu$ m glass beads in thin clay) as a Flush-to-Pipe Loop Volume Ratio Showing Three Complete Flushes 10.10

Figure 10.10. Flush Data for HHM Simulant (100- $\mu \mathrm{m}$ stainless steel in thin clay) as a Flush-toPipe Loop Volume Ratio Showing Three Complete Flushes

Figure 10.11. Flush Data for LLH Simulant (10- $\mu \mathrm{m}$ glass beads in thick clay) as a Flush-to-Pipe Loop Volume Ratio Showing Three Complete Flushes

Figure 10.12. Flush Data for HLH Simulant (10- $\mu \mathrm{m}$ stainless steel in thick clay) as a Flush-toPipe Loop Volume Ratio Showing Three Complete Flushes. Note that the pressure port valves were closed during flush.

Figure 10.13. Flush Data for MMH Simulant (50- $\mu \mathrm{m}$ alumina in thick clay) as a Flush-to-Pipe Loop Volume Ratio Showing Three Complete Flushes. Note that the pressure port valves were closed during flush.

Figure 10.14. Flush Data for LHH Simulant (100- $\mu$ m glass beads in thick clay) as a Flush-toPipe Loop Volume Ratio Showing Three Complete Flushes 10.15

Figure 10.15. Flush Data for HHH Simulant (100 $\mu \mathrm{m}$-stainless steel in thick clay) as a Flush-toPipe Loop Volume Ratio Showing Four Complete Flushes 


\section{Tables}

Table 3.1. Lower Limit on Particle Size for Various Critical-Velocity Correlations ......................3.1

Table 3.2. Critical-Velocity Correlation Rationale for Down Select............................................... 3.1

Table 3.3. Criticisms of Recommended Critical-Velocity Correlations .......................................... 3.2

Table 3.4. Steps Outlined in the 24590-WTP-GPG-M-0058, Rev 0 design guide (Hall 2006)......3.4

Table 4.1. Particle Size and Density Groupings or Bins that Are Significant to Hanford Waste

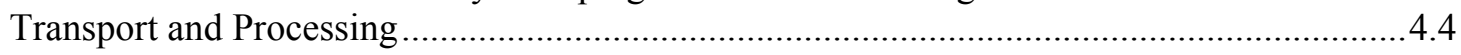

Table 4.2. Test Matrix Combinations ....................................................................................... 4.5

Table 4.3. Test Matrix and Slurry Materials Selected for Critical-Velocity Testing .....................4.7

Table 4.4. Properties of "Low" Rheology Simulant Test Matrix..................................................4.10

Table 4.5. Properties of "Medium" Rheology Simulant Test Matrix ..........................................4.11

Table 4.6. Properties of "High" Rheology Simulant Test Matrix ................................................4.12

Table 7.1. 24590-WTP-GPG-M-0058, Rev 0 design guide (Hall 2006) Critical-Velocity

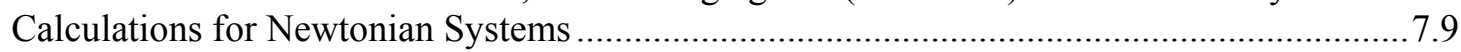





\subsection{Introduction}

The U.S. Department of Energy (DOE) Office of River Protection's Waste Treatment and Immobilization Plant (WTP) will process and treat radioactive waste that is stored in tanks at the Hanford Site. Piping, pumps, and mixing vessels will transport, store, and mix the high-level waste slurries in the WTP.

The WTP pipe-plugging issue, as stated by the External Flowsheet Review Team (EFRT) Executive Summary, is as follows: "Piping that transports slurries will plug unless it is properly designed to minimize this risk. This design approach has not been followed consistently, which will lead to frequent shutdowns because of line plugging" (WTP/CCN 132846). ${ }^{1}$ Additional details relating to the EFRT summary are provided in a supplemental background document (CCN 132847). ${ }^{2}$ The WTP Project is implementing a strategy to address the above EFRT issue identified as "Issue M1-Plugging in Process Piping." Test objectives specified in test specification 24590-WTP-TSP-RT-07-005, Rev. 0 (BNI 2007) state that testing should "Confirm minimum waste velocity and flushing velocity requirements as established by calculations and critical line velocity correlations in the design guide (Hall 2006)."

The tests described herein address the need to perform critical-velocity tests on physical simulants. Critical velocity is defined as the point where a moving bed of particles begins to deposit on the bottom of a straight horizontal pipe during slurry-transport operations. Deposition testing of slurries containing particles with well-characterized size and density suspended in fluids with known rheological properties was performed. The test results provide information to evaluate the conditions that can lead to plugging in pipeline transport operations.

The simulant test particles ranged in density from 2.5 to $8 \mathrm{~g} / \mathrm{cc}$ while the nominal particle size ranged from 10 to $100 \mu \mathrm{m}$. Seventeen tests were conducted with these test particles suspended in three carrier fluids with target Bingham plastic yield-stress values of 0,3 , and $6 \mathrm{~Pa}$.

An experimental flow loop was constructed of 3-inch schedule 40 stainless steel piping with a mixing tank, slurry pump, and instrumentation for determining flow rate and pressure gradient. At the beginning of a test, the slurry flow velocity was nominally set to $8 \mathrm{ft} / \mathrm{sec}$. The flow was then incrementally decreased, and a steady-state pressure gradient was obtained at each flow condition. A rise in pressure gradient as the flow rate drops indicates that the pipe cross-sectional area is filling with moving sediment. This point is referred to as the "critical velocity." This should not be confused with the laminar-toturbulent transition velocity, which is referred to as the "transition velocity."

\footnotetext{
${ }^{1}$ WTP Project Doc. No. CCN 132846 "Comprehensive Review of the Hanford Waste Treatment Plant Flowsheet and Throughput-Assessment Conducted by an Independent Team of External Experts," March 2006. Available online at: http://www.hanford.gov/orp/uploadfiles/Final_ERT\%20Review\%20of\%20EAC.pdf. Accessed 06/13/08.

${ }^{2}$ WTP Project Doc. No. CCN 132847 "Background Information and Interim Reports for the Comprehensive Review of the Hanford Waste Treatment Plant Flowsheet and Throughput-Assessment Conducted by an Independent Team of External Experts," March 2006.
} 
To clear the sediment bed from the system, a flush system modeled after the WTP design (as of August 2007) was installed and tested. This system consists of a pressure vessel where an initial charge of water was placed. The pressure was then increased to a target value, nominally 100 to $110 \mathrm{psig}$. A valve was opened, and the high-pressure water flush removed deposited slurry particles from the pipe loop.

Results from this testing provide an indication of slurry stability as a function of fluid rheological properties and transport conditions. These experimental results are compared to the WTP design guide in an effort to confirm minimum waste-velocity and flushing-velocity requirements as established by calculations and critical line-velocity correlations in the design guide. 


\subsection{Quality Requirements}

PNNL's Quality Assurance Program is based on requirements defined in U.S. Department of Energy (DOE) Order 414.1C, Quality Assurance, and Title 10 of the Code of Federal Regulations (CFR)

Part 830, Energy/Nuclear Safety Management, Subpart A-Quality Assurance Requirements (a.k.a. the Quality Rule). PNNL has chosen to implement the requirements of DOE Order 414.1C and 10 CFR 830, Subpart A by integrating them into PNNL's management systems and daily operating processes. The procedures necessary to implement the requirements are documented through PNNL's Standards-Based Management System.

PNNL implements the RPP-WTP quality requirements by performing work in accordance with the River Protection Project-Waste Treatment Plant Support Program (RPP-WTP) Quality Assurance Plan (RPP-WTP-QA-001, QAP). Work was performed to the quality requirements of NQA-1-1989 Part I, Basic and Supplementary Requirements, NQA-2a-1990, Part 2.7 and DOE/RW-0333P, Rev 13, Quality Assurance Requirements and Descriptions (QARD). These quality requirements are implemented through the River Protection Project-Waste Treatment Plant Support Program (RPP-WTP) Quality Assurance Manual (RPP-WTP-QA-003, QAM). 



\subsection{Background}

Liddell and Burnett (2000) provided a review of critical transport-velocity correlations and models for Hanford tank slurries. They state that each model should be used with systems comparable to the baseline data set for which the model was created. Six primary models were reviewed, and the lower limit of particle size for each model is shown Table 3.1.

Table 3.1. Lower Limit on Particle Size for Various Critical-Velocity Correlations

\begin{tabular}{lc}
\hline \multicolumn{1}{c}{ Model } & Lower Limit of Particle Size \\
\hline Göğ̈̈ş and Kökpınar (1993; 1999, pp. 567-586) & $230 \mu \mathrm{m}$ \\
Gillies and Shook (1991) & $150 \mu \mathrm{m}$ \\
Bae et al. (1991) & $230 \mu \mathrm{m}$ \\
Kim et al. (Stokes Case 1986) & $90 \mu \mathrm{m}$ \\
Oroskar and Turian (1980) & $100 \mu \mathrm{m}$ \\
Toda et al. (1979) & $180 \mu \mathrm{m}$ \\
\hline
\end{tabular}

These correlations were developed for slurries with narrow particle-size distributions (PSDs). Hanford tank wastes consist of a broad distribution of particles with a significant fraction below $100 \mu \mathrm{m}$. Since none of these correlations consider particles significantly smaller than $100 \mu \mathrm{m}$, Liddell and Burnett (2000) conclude that "... there is no published empirical critical-velocity equation that is directly applicable to Hanford tank waste slurries."

For the reasons stated in Table 3.2, Liddell and Burnett (2000) discount the following models from further use for Hanford applications:

Table 3.2. Critical-Velocity Correlation Rationale for Down Select

\begin{tabular}{ll}
\hline \multicolumn{1}{c}{ Model } & Reasons for Not Being Recommended for DOE-EM Applications \\
\hline Göğüş and Kökpınar (1993, 1999) & - Minimum particle size factor of two too large \\
& - Similar to Oroskar and Turian (1980) and not extensively tested \\
Bae et al. (1991) & - Minimum particle size factor of two too large \\
& - No pipe size dependence \\
Kim et al. (Stokes Case, 1986) & - Assume Stokes or intermediate-range hindered settling \\
& - Need to adapt to settling under turbulent flow conditions \\
Toda et al. (1979) & - Several typographical errors in mathematical notation \\
\hline
\end{tabular}

With these limitations, Lidell and Burnett (2000) state that the Oroskar and Turian (1980) and Gillies and Shook (1991) models are the most likely candidates for slurry-transport design at the Hanford site. However, there are issues with using these equations for transport of Hanford HLW slurries. These issues are stated in Table 3.3 . 
Table 3.3. Criticisms of Recommended Critical-Velocity Correlations

\begin{tabular}{ll}
\hline Model & Notes \\
\hline Oroskar and Turian (1980) & - Applied to size distributions much different than those from \\
& which it was derived \\
& - Monosized distributions \\
& - Lower limit only $100 \mu \mathrm{m}$ \\
\hline Gillies and Shook (1991) & Developed for distributions consisting of fine-particle carrier \\
& - If dense particles are present in carrier fluid, they could settle \\
& - $74-\mu$ m cut point is arbitrary. \\
\hline
\end{tabular}

Liddell and Burnett (2000) conclude the following:

The equations of Oroskar and Turian (1980) and Gillies and Shook (1991) are the likeliest candidates for use in transfer pipeline design. The Gillies and Shook expression was developed for broadly graded slurries consisting of a mixture of particles at least $150 \mu \mathrm{m}$ in diameter and much smaller particles in a pseudohomogenous vehicle. As such it is probably capable of better predicting the behavior of tank solids than the equation of Oroskar and Turian, which is the result of a multilinear regression of a large amount of literature data, much of it for monosized particles.

However, neither expression has been validated on a broadly graded slurry with a size distribution that is continuous and similar to Hanford tank waste. Experimental test work should be performed to evaluate how accurately these two equations represent the critical transport velocity of tank solids simulants.

Often the rheology of the fine-particle transport "vehicle" will be non-Newtonian in nature. This scenario is commonplace in Hanford tank waste applications. Yield-stress forces can dampen the evolution of eddies in pipeline flow and hamper the onset of turbulent flow. Turbulent flow is a precursor requirement for the application of all of the equations discussed above. Shook et al. (2002) state that "There have been many experimental investigations of non-Newtonian slurries in laminar and turbulent flow and deposition is often observed as the laminar flow condition is approached." They go on to state, "The exceptions to this behavior, i.e., where deposition does not occur in laminar flow, seem to be associated with flows of very high pressure gradients.... It should be noted however that particles may settle very slowly in a laminar slurry flow so that long test pipelines may be required to detect deposition." A rule of thumb is that a pressure gradient of about $2 \mathrm{kPa} / \mathrm{m}$ is required to remove a stationary deposit in laminar flow (Shook et al. 2002).

Physically, this means that if a bed of solids is deposited in the pipe, a pressure drop of about $9 \mathrm{psi} /$ $100 \mathrm{ft}$ of pipe is required to move any appreciable amount of solids through the pipe system. Below this pressure-gradient threshold, friction dominates, and the bed remains stationary, although flow can occur over the top of the particle bed. 
Based on this information, there are two primary questions regarding critical-velocity calculations for WTP application:

1. How do the correlations perform with size and density combinations anticipated to be present in the Hanford tank waste sludge (i.e., particles $100 \mu \mathrm{m}$ and lower with densities ranging from 2 to $10 \mathrm{~g} / \mathrm{cc})$ ?

2. How does non-Newtonian rheology affect these design correlation predictions?

\subsection{WTP Design Guide for Critical Velocity}

The WTP design authority wrote a "design guide" discussing the critical velocity of Newtonian fluids in straight, horizontal piping (24590-WTP-GPG-M-0058, Rev 0; Hall 2006). It recommends how critical deposition-velocity calculations should be made for the WTP with the Oroskar and Turian (1980) correlation.

$$
V_{\text {OT }}=\sqrt{g d(S-1)}\left[1.85 C_{c}^{0.1536}\left(1-C_{c}\right)^{0.3564}\left(\frac{D}{d}\right)^{0.378}\left(\frac{\rho_{f} D \sqrt{g d(S-1)}}{\mu_{f}}\right)^{0.09} \chi^{0.3}\right]
$$

where $\quad V_{O T}=$ the Oroskar and Turian $(1980)$ critical velocity $(\mathrm{m} / \mathrm{s})$

$g=$ gravitational acceleration $\left(\mathrm{m} / \mathrm{s}^{2}\right)$

$D=$ pipe inner diameter $(\mathrm{m})$

$d=$ particle diameter $(\mathrm{m})$

$S=$ ratio of the coarse solid density to carrier fluid density, $\rho_{S} / \rho_{f}$

$C_{c}=$ the coarse particle volume fraction (i.e., particles exceeding $74 \mu \mathrm{m}$ )

$\rho_{f}=$ carrier fluid, including fines density $\left(\mathrm{kg} / \mathrm{m}^{3}\right)$

$\mu_{f}=$ carrier fluid dynamic viscosity $(\mathrm{Pa} \cdot \mathrm{s})$

$\chi=$ hindered settling factor.

The design guide specifies a homogeneity criterion to determine the fraction of coarse particles that will settle in a slurry system. The volume fraction of the coarse settling particles is then used as the concentration term in Equation 3.1. For fine particle slurries, the fraction of coarse particles will often be at or near zero, resulting in an Oroskar and Turian equation prediction of nearly zero velocity. In these situations, the so-called "limiting critical velocity," which is based on the Thomas (1979) equation, becomes the dominate design-guide output.

The Thomas (1979) equation is also used in the design guide to overcome the 100 - $\mu \mathrm{m}$ lower limit on particle size for the Oroskar and Turian (1980) correlation. The Thomas (1979) equation was correlated on data from smaller particles. The basis for the correlation is that these particles settle to the bottom of the pipe and are conveyed in a sliding bed regime via the laminar sub-layer. Although the literature has limited information on particles with these size and density characteristics, Thomas (1979) formed his correlation on two silica/water systems with particle sizes of 17 and $26 \mu \mathrm{m}$ and compared his predictions over a wide 
range of particle densities and achieved reasonable results. Particles discussed in this study include talc, barium sulfate, iron powder, and tungsten. In the design guide, the Thomas (1979) equation is used as:

$$
V_{T}=9.0\left(\frac{g \mu_{W}(S-1)}{\rho_{f}}\right)^{0.37}\left(\frac{D \rho_{f}}{\mu_{W}}\right)^{0.11}
$$

where $V_{T}$ is the Thomas (1979) critical velocity $(\mathrm{m} / \mathrm{s})$, and $\mu_{W}$ is the carrier fluid dynamic viscosity (assumed to be water; Pa.s). Since the viscosity of water is used in Equation 3.2, the predicted velocities will be lower than those predicted using the viscosity of the actual carrier fluid. For this reason, the design guide considers this to be a lower "limiting critical velocity" of the slurry stream.

The design guide is written in steps indicated in Table 3.4.

Table 3.4. Steps Outlined in the 24590-WTP-GPG-M-0058, Rev 0 design guide (Hall 2006)

\begin{tabular}{clc}
\hline Step Number & \multicolumn{1}{c}{ Description } & Variable Defined \\
\hline 1 & Determine PSD & $d$ \\
2 & Determine the upper limit (UL) particle-density distribution (PDD) & $\rho_{S}$ \\
3 & Determine the solids volume fraction & $C_{c}$ \\
4 & Determine the carrier liquid density & $\rho_{f}$ \\
5 & Determine the carrier liquid viscosity & $\mu_{f}$ \\
6 & Determine the hindered settling factor & $\chi$ \\
7 & Calculate the critical velocity of the fluid stream & $V_{O T}$ \\
8 & Calculate the limiting critical velocity of the fluid stream & $V_{T}$ \\
9 & Apply a design margin & $M$ \\
\hline
\end{tabular}

The details for determining each of these values are summarized for each step in the design guide as follows:

1. The design guide specifies the use of the $d_{95}$ particle diameter for line transfers and the $d_{99}$ particle diameter for line flushes.

2. The design guide specifies the use of the $d_{95}$ density; this term is ambiguous and needs clarification in the design guide. Nonetheless, this value is interpreted to mean the density of the $d_{95}$ particle. This density value is irrelevant for critical velocity calculations. Often this value is unknown, and Equation 1 of the 24590-WTP-GPG-M-0058, Rev 0 design guide (Hall 2006) will be used for design purposes. This equation calculates an average or composite density of all solids in the slurry.

3. For Newtonian slurries, the design guide specifies to determine the fraction of particles larger than $74 \mu \mathrm{m}$. These particles are considered to be heterogeneous or settling fraction of the slurry. Particles smaller than $74 \mu \mathrm{m}$ are considered to be homogeneous or non-settling. The homogeneous fraction of the slurry is denoted as $\eta_{\text {homo }}$. The coarse volume fraction of solids is determined as $C_{c}=\left(1-\eta_{\text {homo }}\right) \cdot C_{s}$ 
and the fine fraction of solids is determined by $C_{f}=\eta_{\text {homo }} \cdot C_{s}$. In both of these cases, $C_{s}$ is the total undissolved solids in the slurry. For non-Newtonian fluid with a yield stress, Darby (2000) suggests that yield stress can support the particles as they are being transported. In this case, a stability criterion parameter, $Y$, is calculated using the equation shown below.

$$
Y=\frac{\tau_{y}}{g d\left(\rho_{s}-\rho_{m}\right)}
$$

where $Y$ is the yield-stress stability parameter. If greater than 0.2 , the particle is supported by yield stress and considered homogeneous or non-settling, $\tau_{y}$ is the yield stress of the fluid (Pa), and $\rho_{m}$ is the slurry mixture density $\left(\mathrm{kg} / \mathrm{m}^{3}\right)$.

4. The design guide specifies the calculation of the density of the slurry resulting from the combination of interstitial liquid and the homogeneous particles.

5. The design guide specifies several equations to estimate the viscosity of the carrier slurry. Among these equations are several that describe a relationship between the homogeneous solids fraction and the slurry viscosity.

$$
\mu_{f}=2.0\left\{1+2.5 C_{f}+10.05 C_{f}^{2}+1.3\left[\exp \left(17 C_{f}\right)-1\right]\right\}(0.735)
$$

where $\mu_{f}$ is the carrier fluid dynamic viscosity $(\mathrm{Pa} \cdot \mathrm{s})$, and $C_{f}$ is the fine or homogeneous particle volume fraction (i.e., particles below $74 \mu \mathrm{m}$ ).

6. The design guide specifies that the hindered settling factor is typically 0.95 for Hanford tank sludge.

7. The variables defined in steps 1 to 6 are used as inputs to the Oroskar and Turian (1980) criticalvelocity correlation.

8. The variables defined in steps 1 to 6 are used as inputs to the Thomas (1979) critical-velocity correlation.

9. A minimum design margin of $30 \%$ will be applied to the largest critical velocity between $V_{O T}$ and $V_{T}$ determined in steps 7 and 8 . The resulting value is the recommended design pipeline superficial velocity.

The design guide raises several technical questions that were addressed through the testing described in this report. These issues are described as follows:

1. Role of Density in Calculations-The $d_{95}$ particle size and the average bulk density of the slurry particles are typical inputs for the design correlations. Turian et al. (1987) state the following:

For slurries comprised of large non-colloidal particles, the critical velocity is virtually independent of particle size. The analytical result due to Oroskar and Turian (1980) indicates that $v_{c}$ is independent of $d$, while analysis of the experimental data suggests that $v_{c}$ is a very weak function of $d$.

Consequently, the design guide directs WTP engineers to choose a conservative value, $d_{95}$ or $d_{99}$, for particle size in the empirical version of the Oroskar and Turian equation (1980), which has lesser influence on critical velocity relative to other variables (in particular, particle density). Conversely, the design guide directs WTP design engineers to choose a low value on particle density (i.e., the 
average particle density of the slurry or the density of the $d_{95}$ particle), which has a much greater impact on critical velocity.

The design guide specifies the use of the $d_{95}$ density; this term is ambiguous and needs clarification in the design guide. Nonetheless, this value is interpreted to mean the density of the $d_{95}$ particle. This density value is irrelevant for critical velocity calculations. Often this value is unknown, and Equation 1 of the 24590-WTP-GPG-M-0058, Rev 0 design guide (Hall 2006) will be used for design purposes. This equation calculates an average or composite density of all solids in the slurry. Hanford tank waste has a broad distribution of expected densities (2 to $10 \mathrm{~g} / \mathrm{cc})$. Therefore, the combination of a small particle size and high density may require higher transport velocities than that calculated with the $d_{95}$ particle size and $d_{95}$ particle density properties.

For example, in a given waste slurry, the $d_{50}$ was $5 \mu \mathrm{m}$, and the $d_{95}$ was $100 \mu \mathrm{m}$. Based on the overall particle diameter exponent of 0.167 in Equation 3.1, use of the $d_{95}$ instead of $d_{50}$ increases the design critical velocity by a factor of 1.65 . Since this is a significant increase in design velocity, this is a conservative assumption. While the exponent on particle size may be low, this protocol does have a significant conservative effect when the PSDs are broad, such as with tank waste slurries.

Now consider a waste with an average particle density of $3.25 \mathrm{~g} / \mathrm{cm}^{3}$ with $10 \%$ of the volume of the slurry particles being dense $10 \mathrm{~g} / \mathrm{cm}^{3}$ particles and the remaining $90 \%$ having a density of $2.5 \mathrm{~g} / \mathrm{cm}^{3}$. Neglecting differences in particle size, the actual velocity to prevent deposition would be a factor of 1.9 times higher than predicted by the average particle density. Both of these examples illustrate that the design guide should emphasize a conservatively high diameter and particle density.

2. Homogeneous Fraction Concept-The concept of a homogeneous and heterogeneous fraction in the slurry being conveyed is presented in the model by Gillies and Shook (1991). In the design guide, this concept is then applied to the Oroskar and Turian (1980) correlation. Liddell and Burnett (2000) point out that the 74- $\mu \mathrm{m}$ cut-point (i.e., the opening for a 200 -mesh sieve) to distinguish between heterogeneous and homogeneous solids is arbitrary. They point out that if particles less than $74 \mu \mathrm{m}$ are dense enough they are expected to settle.

3. Use of RPP-9805 Viscosity Prediction-When presenting his viscosity correlation for Hanford tank waste Jewett et al. (2002) make the following statement:

The viscosity correlations presented above have some weaknesses. One weakness is the relatively small number of tanks for which viscosities have been obtained for various defined solids loadings. Data for additional tanks would improve the quality of the final correlation. Furthermore, most viscosities used in this correlation were "apparent" and are equal to or greater than the true viscosity of the fluid.

The basis for the Jewett viscosity correlation is a correlation for Newtonian, non-interacting, spherical, dilute, uniform particles developed by DG Thomas (1965). The Hanford tank waste consists of non-Newtonian, interacting, non-spherical, non-uniform particles. The particle shape and uniformity attributes of the Hanford tank sludges are discussed in detail by Wells et al. (2007). A sister report by Poloski et al. (2007) discusses the non-Newtonian fluid properties of the Hanford tank sludge.

These differences in basic assumptions between ideal systems (considered to be non-interacting, spherical, dilute, uniform particles) and real systems (with complex particle shapes, particle size distributions, and interparticle forces) result in non-Newtonian Hanford tank sludge behavior. NonNewtonian fluids are defined as fluids whose viscosity varies with the applied shear rate. The nonNewtonian term "apparent viscosity" is defined as the viscosity under a specific shear-rate condition and is used for pseudo-plastic or visco-plastic fluids. Jewett recognized that the Hanford tank sludge is non-Newtonian and applied the DG Thomas correlation (1965) that was augmented with a term that 
accounts for the average shear rate in a piping system. The technical basis for such an approach is not strongly supported by the literature. The literature contains several empirical relations between particle concentration, interparticle attraction, and non-Newtonian fluid properties. Further detail on these concepts can be found in a textbook edited by Brown and Heywood (1991). A diagram illustrating various classes of fluid rheology and how they relate to the Jewett et al. (2002) correlation and experimental rheological measurements of Hanford waste was adapted from Brown and Heywood (1991) and is shown in Figure 3.1.

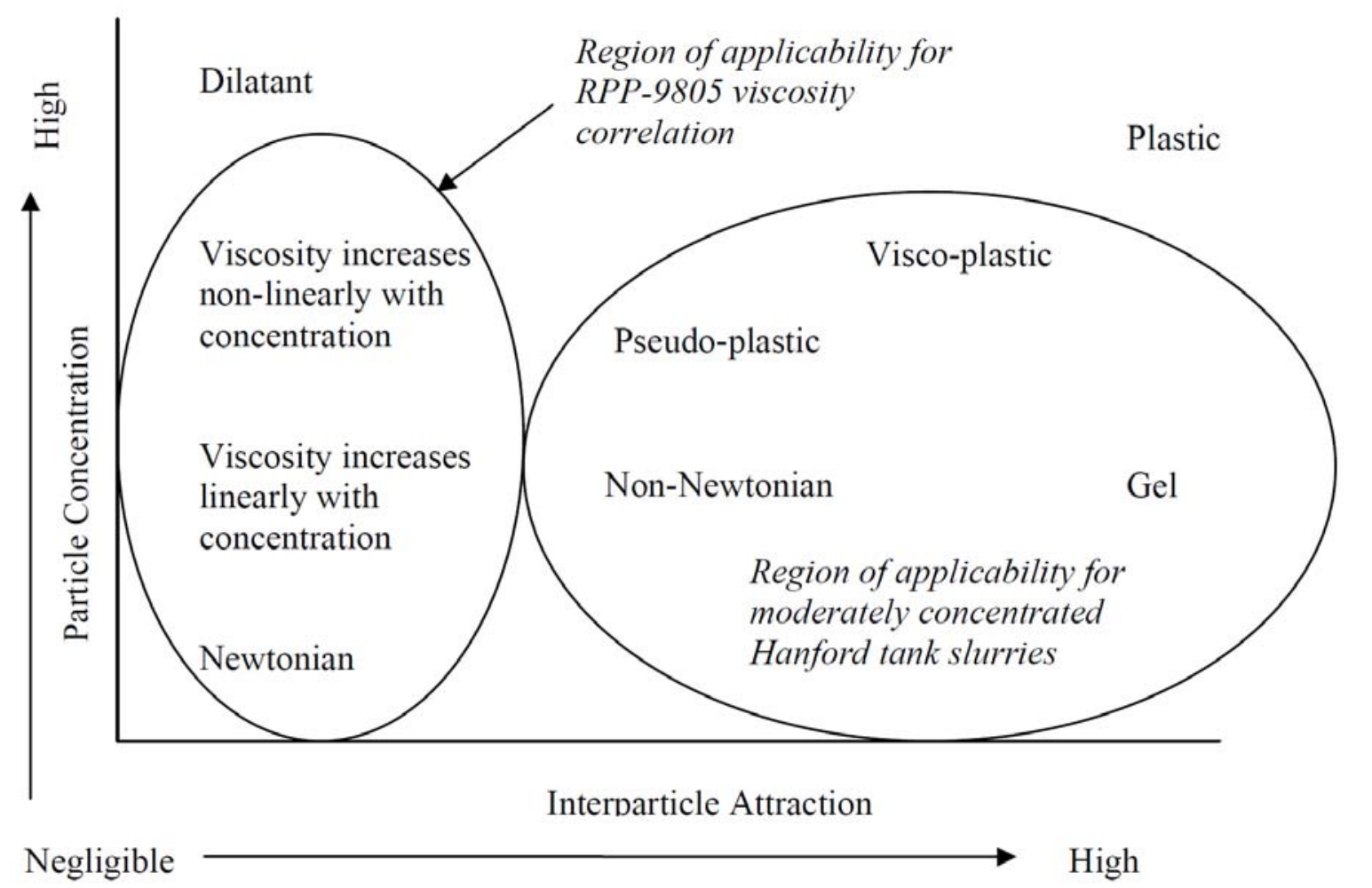

Figure 3.1. Effects of Particle Concentration and Interparticle Attraction on Fluid Rheology (adapted from Brown and Heywood (1991)

4. Design for non-Newtonian Flows-As discussed above, Hanford tank slurries are non-Newtonian at moderate particle concentrations. The critical-velocity design guide (24590-WTP-GPG-M-0058, Rev 0 ; Hall 2006) is limited to considering only Newtonian fluids. However, the threshold particle concentration where non-Newtonian behavior begins depends on the interparticle forces present in the slurry. For some waste slurry conditions, non-Newtonian behavior is present at low particle concentrations while under different conditions, non-Newtonian behavior only exists under high particle concentrations. The factors that influence interparticle forces are complex and include several chemical factors, including $\mathrm{pH}$, surface chemistry, ionic strength, particle size, particle shape, etc. Undoubtedly, the WTP will process large quantities of non-Newtonian fluids (Poloski et al. 2003, 2006, 2007), and understanding how critical velocity is influenced by non-Newtonian properties is important to the success of the Waste Treatment and Immobilization Plant mission. 


\subsection{Test Strategy}

An experimental program was implemented to answer the four technical questions raised in Section 3.1 and to address the test objective specified in test specification 24590-WTP-TSP-RT-07-005, Rev. 0 (BNI 2007) stating that testing should "Confirm minimum waste velocity and flushing velocity requirements as established by calculations and critical line velocity correlations in the design guide."

A suite of simulants was developed from actual waste particle size and density information (Wells et al. 2007) and actual waste rheological property data (Poloski et al. 2007). These simulants were designed to test the expected range of particle size and density while being in turbulent flow at the design-basis velocities of 4 to $6 \mathrm{ft} / \mathrm{sec}$. This requirement restricts the range of experimental rheological properties from the upper bounds approaching 30-Pa Bingham yield stress found in Poloski et al. (2003, 2006). Fifteen simulants were developed with test particles ranging in density from 2.5 to $8 \mathrm{~g} / \mathrm{cc}$ while the nominal particle size ranged from 10 to $100 \mu \mathrm{m}$. Seventeen tests were conducted with these test particles suspended in three carrier fluids with target Bingham plastic yield-stress values of 0, 3, and $6 \mathrm{~Pa}$. Details of simulant selection rationale and physical properties can be found in Section 4.0.

An experimental flow loop was constructed of approximately 100 feet of 3 -inch schedule 40 piping taken from the WTP excess yard (see Figure 3.2). The flow loop components included a four-baffled, 400 -gallon mixing tank with a 25 -inch pitched-blade impeller. A Georgia Iron Works slurry pump, a 150-psig, 400-gallon pneumatic flush system, and a 1,000 gallon capture tank complete the flow loop system. The flow loop also has instrumentation for determining flow rate with inlet and outlet Coriolis flow meters. The pressure gradient was measured with a series of three differential pressure transducers. The Particle size chord-length distribution was measured with a Lasentec ${ }^{\mathrm{TM}}$ focused beam reflectance measurement (FBRM) sensor. Lastly, a cross-sectional pipe tomogram of slurry conductivity was measured using electrical resistance tomography. Details of test apparatus can be found in Section 5.0. 


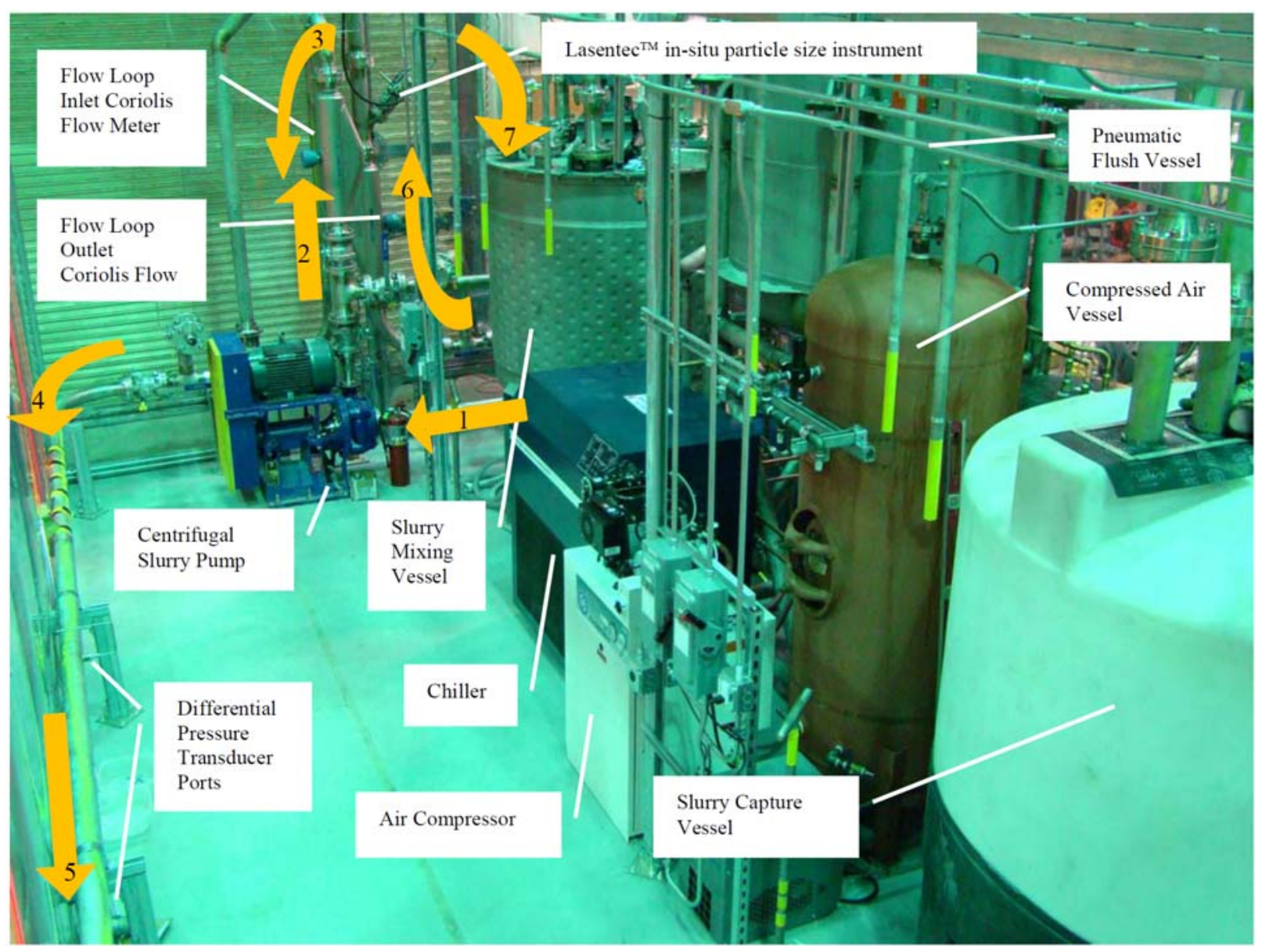

Figure 3.2. Photograph of Flow Loop System with Major Equipment Identified; Arrows and Numbers Indicate Normal Operation Flow Direction

At the beginning of a test, the slurry flow velocity was nominally set to $8 \mathrm{ft} / \mathrm{sec}$. The flow was then incrementally decreased, and a steady-state pressure gradient was obtained at each flow condition. A rise in pressure gradient as the flow rate drops indicates that the pipe cross-sectional area was filled with sediment. This point is referred to as the "critical velocity." The critical velocity was defined as the point where a moving bed of particles begins to deposit on the bottom of a straight horizontal pipe during slurry-transport operations. This should not be confused with the laminar-to-turbulent transition velocity, which was referred to as the "transition velocity." Details of the test procedure can be found in Section 6.0.

Critical-velocity tests were then performed on the fifteen physical simulants. Results were grouped according to Newtonian, which accounted for 5 simulants, and non-Newtonian fluid rheology, which accounted for 10 simulants. The midpoint particle-size, particle-density, and rheology simulant was replicated twice to determine the degree of repeatability of the system. Newtonian and non-Newtonian experimental critical velocities and a comparison to the 24590-WTP-GPG-M-0058, Rev 0 design guide (Hall 2006) minimum velocity are presented in Sections 7.0 and 8.0, respectively. 
The deposition of particles in these systems was shown to be a strong function of slurry rheological properties. Since this topic was not considered in the design guide, an analysis of the Newtonian and non-Newtonian data was performed. The results of this analysis form the basis for a series of recommended WTP design-guide equations. The critical-velocity data analysis and recommendations can be found in Section 9.0.

To clear the sediment bed from the system, a flush system modeled after the WTP design (as of August 2007) was installed and tested. This system consists of a pressure vessel where an initial charge of water was placed. The pressure was then increased to a target value, nominally 100 to $110 \mathrm{psig}$. A valve was opened, and the high-pressure water flush removed deposited slurry particles from the pipe loop. The 24590-WTP-GPG-M-0058, Rev 0 design guide (Hall 2006) specified a flush volume of 1.5 to 1.7 times the piping volume for removing the sediment bed. This criterion is analyzed in Section 10.0. Finally, the testing conclusions are summarized in Section 11.0. 


\subsection{Test Materials}

The technical approach to answer the questions posed in Section 3.1 involved building a full-scale pipeline system and measuring the critical velocities on test materials. These slurries were designed to possess particle-size, density, and non-Newtonian rheological property values similar to those expected under WTP operating conditions. The basis for the particle-size, density, and rheological property values tested is explained in detail in this section. A description of the slurry physical and rheological properties of these test materials is also provided.

\subsection{Expected Range of Hanford Particle Density}

A WTP feed vector from the Hanford Tank Waste Operation Simulator (HTWOS) case "RebaselineRun_04-26-06" named "batches-delivered-to-wtp" with a run date of 6/30/2006 under fully water-washed conditions was used to assess the feed variability of the slurries going to WTP. These data provide the fraction of a source Hanford tank that comprises a batch of material to be fed to the WTP. Low-activity waste (LAW) batches were not considered in this analysis. The elemental species in the solid phase of this feed vector were assigned chemical compounds consistent with WTP-RPT-153 Rev 0 (Wells et al. 2007). For example, aluminum present in the solids was assumed to be $\mathrm{Al}(\mathrm{OH})_{3}$ or gibbsite; iron, zirconium, bismuth, and uranium were assigned the chemical compounds $\mathrm{Fe}_{2} \mathrm{O}_{3}, \mathrm{ZrO}_{2}, \mathrm{Bi}_{2} \mathrm{O}_{3}$, and $\mathrm{Na}_{2} \mathrm{U}_{2} \mathrm{O}_{7}$. While compounds outside of the set discussed in the WTP-RPT-153, Rev 0 (Wells et al. 2007) report are present, the solid-phase species selected are the dominate compounds present in the tank waste.

Since aluminum species are the dominant material in the tank waste solids, these volumes were normalized to the volume of gibbsite in each batch. The volume ratio of the slurry particle volume to gibbsite volume entering the WTP according is shown in Figure 4.1 (top). The post-leaching case for HLW pretreated sludge (after leach factors are applied, and the sludge is renormalized to the new quantity of aluminum species) is provided in the bottom plot of Figure 4.1 .

These data show that the majority of the particles being fed to the WTP will consist of particles in the 2 to $4 \mathrm{~g} / \mathrm{cm}^{3}$ range as aluminum species. Some batches will periodically consist of a relatively high fraction of particles in the 4 to $6 \mathrm{~g} / \mathrm{cm}^{3}$ density range. This can be seen in the first 5 years of the WTP campaign. On a volume basis, only a small portion of the waste feed is predicted to have solid densities exceeding $8 \mathrm{~g} / \mathrm{cm}^{3}$; however, on a mass basis, approximately $2 \%$ of the waste feed is predicted to have solid densities exceeding $8 \mathrm{~g} / \mathrm{cm}^{3}$ (Wells et al. 2007).

The majority of the particles are still in the 2 to $4 \mathrm{~g} / \mathrm{cm}^{3}$ range for the post caustic-leaching case. However, some batches are expected to consist predominately of particles in the 4 to $6 \mathrm{~g} / \mathrm{cm}^{3}$ range. This can be seen in batches received the first 5 years and the final 5 years of WTP operations. In the years during 2019, 2022, 2029, 2031, and 2034, HLW pretreated sludge batches with a significant volume of particles above $8 \mathrm{~g} / \mathrm{cm}^{3}$ are expected to be processed.

In summary, three dominant density bins of 2 to 4,4 to 6 , and $>8 \mathrm{~g} / \mathrm{cm}^{3}$ of particle density will be processed in the WTP. Consequently, this test program was designed around the simulant particles with density groupings labeled "Low," "Medium," and "High" for target particle densities in the 2 to 4, 4 to 6, and 8 to $10 \mathrm{~g} / \mathrm{cm}^{3}$, range, respectively. 

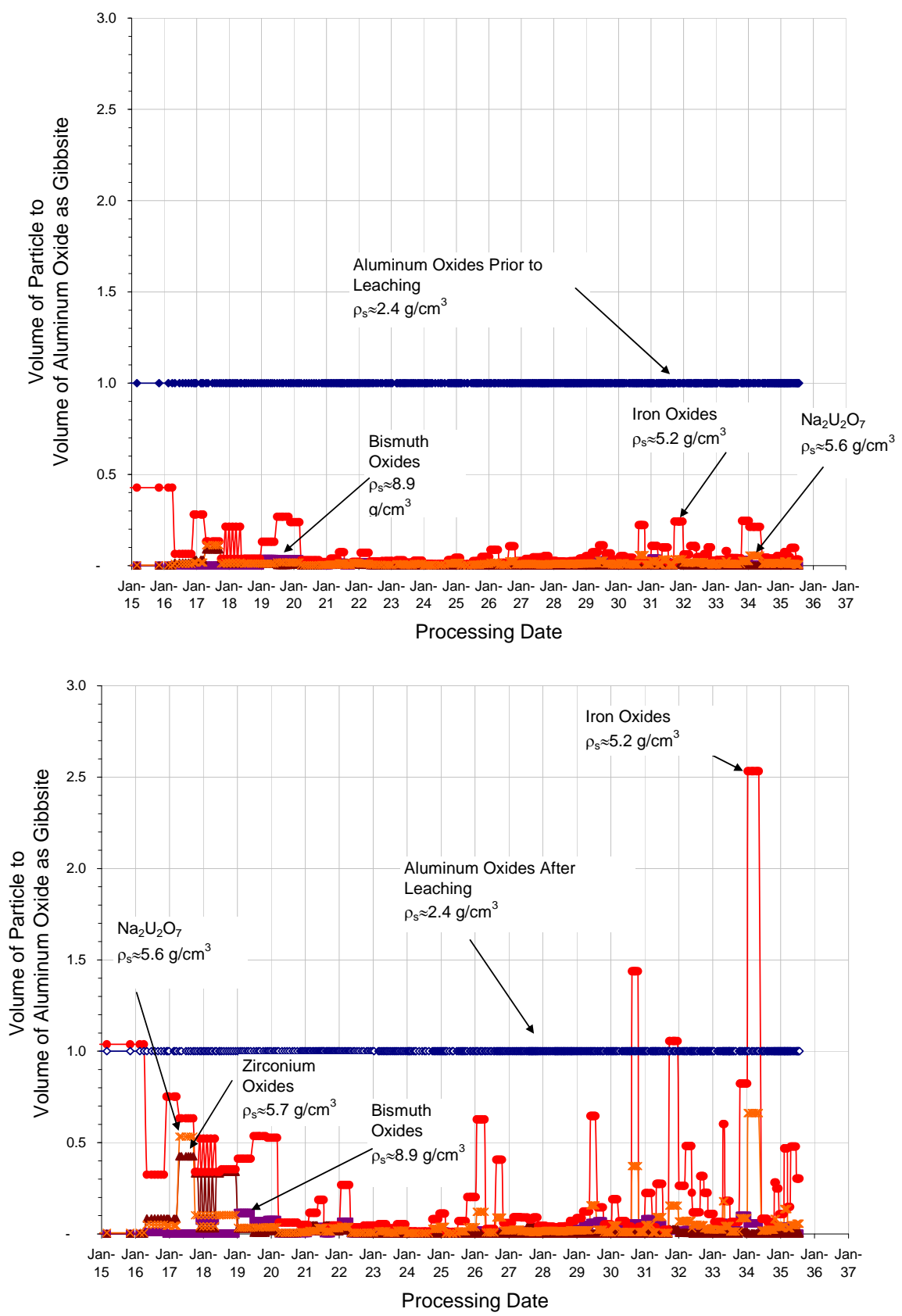

Figure 4.1. Top: Timeline Showing the Ratio of Volume of Slurry Particulate Compounds to the Volume of Aluminum Oxide as Gibbsite Prior to Leaching.

Bottom: Timeline showing the ratio of volume of slurry particulate compounds to the volume of Aluminum Oxide as Gibbsite After Leaching.

Note that this plot is unreviewed and is provided "for information only."

\subsection{Expected Range of Hanford Particle Size}

Report WTP-RPT-153 Rev. 0 (Wells et al. 2007) provides a representative, composite PSD for the "as-retrieved" insoluble solids comprising sludge layers at the Hanford Tank Farms. Two composite PSDs are shown in Figure 4.2;1) a "sonicated" composite PSD ("all sonic") and 2) a minimal 
disturbance, un-sonicated composite PSD ("all md"). Both of these curves are similar; however, the sonicated PSD is shifted to lower particle diameters, likely as a result of particle flocs that are broken down and disrupted by sonication.

Particles less than 3- $\mu \mathrm{m}$ can be considered fines. The range of sizes spanning 3 to 10,10 to 50 , and 50 to $100 \mu \mathrm{m}$ can be considered "Low," "Medium," and "High" particle sizes, respectively. From the cumulative size distribution, the fines are about $30 \%$ of the particles, the "Low" size particles are about $40 \%$ of the particles, "Medium" size particles are about $25 \%$ of the particles, and "High" size particles are about $5 \%$ of the particles by volume.

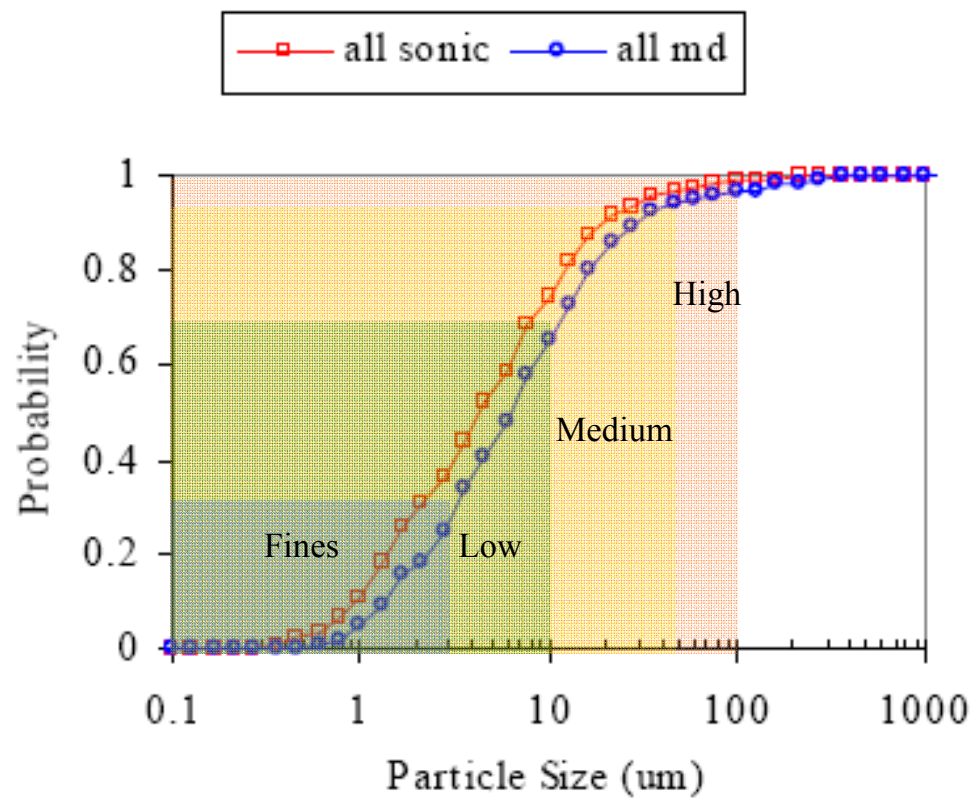

Figure 4.2. PSD of the Composite Hanford Tank Farm Slurries Reported in WTP-RPT-153, Rev. 0 (Wells et al. 2007); Distributions for minimal disturbance ("all md") and sonicated ("all sonic") waste states are given. Highlighted Regions Show the Binning Strategy Used for Simulant Design.

\subsection{Expected Range of Hanford Sludge Rheology}

Report WTP-RPT-154, Rev. 0 (Poloski et al. 2007) provides an expected range of Hanford slurry rheology. The Hanford sludges were shown to behave as Bingham plastic material. The degree of the non-Newtonian behavior in this model is characterized by the Bingham yield-stress parameter. The maximum measured values of Bingham yield stress for Hanford sludge samples are shown in Figure 4.3. Since the critical-velocity equations are derived for Newtonian, turbulent flow conditions, only moderate values of yield stress were selected for the experimental matrix. A yield stress of zero, or a Newtonian fluid, was selected as the "Low" rheology condition. "Medium" and "High" rheology conditions were selected at $3 \mathrm{~Pa}$ and $6 \mathrm{~Pa}$ yield stress, respectively. Several tank samples had Bingham yield-stress values that exceed $6 \mathrm{~Pa}$ and even approach $40 \mathrm{~Pa}$. However, the EFRT has stated "for upper-bound nonNewtonian fluids, the project has addressed potential plugging issues in an acceptable manner". The flow of these fluids in the 4- $\mathrm{ft} / \mathrm{sec}$ velocity range is expected to be laminar. A rheology primer is attached as Appendix A. 


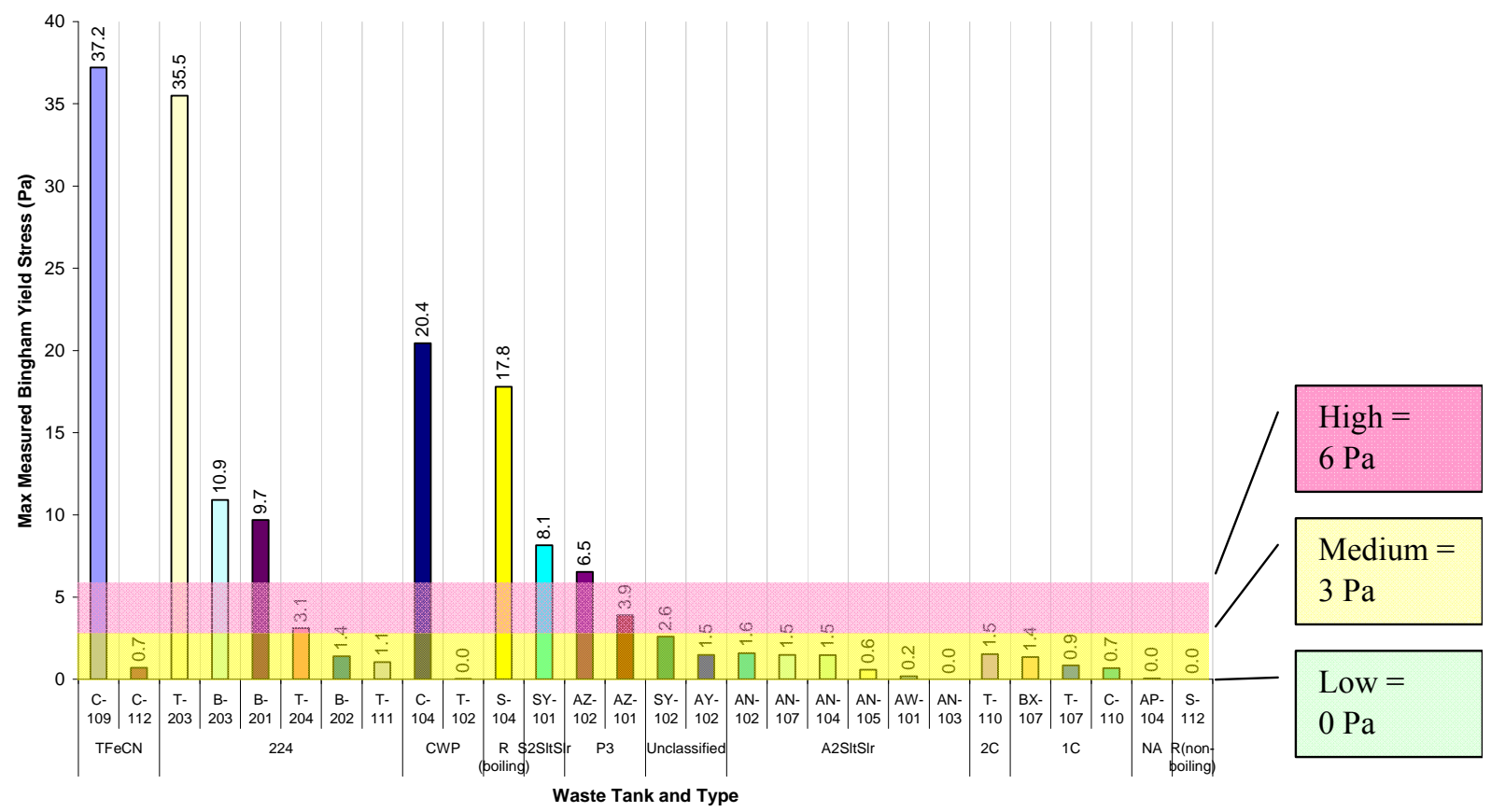

Figure 4.3. Maximum Measured Bingham Yield Stress for Various Hanford Tanks and Waste Types

\subsection{Experimental Test Matrix}

The expected Hanford slurry density and particle-size bins can be combined to form an experimental test space of size and density. This test space is shown in Table 4.1. To verify that a size ratio of 1:10 is maintained for the combination of fine and low particle size, targets of $1 \mu \mathrm{m}$ and $10 \mu \mathrm{m}$ are chosen, respectively. A fines density is chosen in the range of 2 to $3 \mathrm{~g} / \mathrm{cm}^{3}$ for the purpose of mimicking the density of the aluminum compounds, such as gibbsite and boehmite, which dominate the tank waste solids composition. The volume ratio of the fines to component particles, overall slurry solids concentration, the fines composition, and interstitial liquid chemistry were adjusted to achieve rheological targets discussed later in this document. Table 4.2 shows potential test combinations for singlecomponent particle size, density, and fluid rheology. The test conditions are discussed in Section 4.6 of this document.

Table 4.1. Particle Size and Density Groupings or Bins that Are Significant to Hanford Waste Transport and Processing

\begin{tabular}{|c|c|c|c|c|}
\hline Particle Size $\rightarrow$ & Low & Medium & High & Fines \\
\hline Density $\downarrow$ & Nominal: $10 \mu \mathrm{m}$ & Nominal: $50 \mu \mathrm{m}$ & Nominal: $100 \mu \mathrm{m}$ & Nominal: $1 \mu \mathrm{m} \quad 2-3 \mathrm{~g} / \mathrm{cm}^{3}$ \\
\hline $\begin{array}{l}\text { Low } \\
2 \text { to } 4 \mathrm{~g} / \mathrm{cm}^{3}\end{array}$ & {$[\mathrm{~L}, \mathrm{~L}]$} & {$[\mathrm{L}, \mathrm{M}]$} & {$[\mathrm{L}, \mathrm{H}]$} & \multirow{3}{*}{$\begin{array}{c}\text { Purpose is to adjust } \\
\text { rheological properties } \\
\{\mathrm{L}, \mathrm{M}, \mathrm{H}\}\end{array}$} \\
\hline $\begin{array}{l}\text { Medium } \\
4 \text { to } 6 \mathrm{~g} / \mathrm{cm}^{3}\end{array}$ & {$[\mathrm{M}, \mathrm{L}]$} & {$[\mathrm{M}, \mathrm{M}]$} & {$[\mathrm{M}, \mathrm{H}]$} & \\
\hline $\begin{array}{l}\text { High } \\
8 \text { to } 10 \mathrm{~g} / \mathrm{cm}^{3}\end{array}$ & {$[\mathrm{H}, \mathrm{L}]$} & {$[\mathrm{H}, \mathrm{M}]$} & {$[\mathrm{H}, \mathrm{H}]$} & \\
\hline $\begin{array}{l}\mathrm{L}-\text { Low, } \mathrm{M}-\mathrm{M} \\
\text { Shaded cells are }\end{array}$ & $\begin{array}{l}\mathrm{m}, \mathrm{H}-\text { High } \\
\text { ded in reduced }\end{array}$ & al design. & & \\
\hline
\end{tabular}


Table 4.2. Test Matrix Combinations

\begin{tabular}{ccccc}
\hline Test Configuration & Acronym & Particle Density & Particle Size & Fluid Rheology \\
\hline 1 & LLL & Low & Low & Low \\
2 & HLL & High & Low & Low \\
3 & MML & Medium & Medium & Low \\
4 & LHL & Low & High & Low \\
5 & HHL & High & High & Low \\
6 & LLM & Low & Low & Medium \\
7 & HLM & High & Low & Medium \\
8 & MMM & Medium & Medium & Medium \\
9 & LHM & Low & High & Medium \\
10 & HHM & High & High & Medium \\
11 & LLH & Low & Low & High \\
12 & HLH & High & Low & High \\
13 & MMH & Medium & Medium & High \\
14 & LHH & Low & High & High \\
15 & HHH & High & High & High \\
\hline
\end{tabular}

\subsection{Rationale for Simulant Selection}

Two primary approaches for simulant development have been used historically at the Hanford site. Chemical simulants are designed to match the chemical speciation of a tank sludge with a lesser emphasis on matching the physical properties of the material. Physical simulants do not match the waste chemical speciation, but they possess similar physical properties (e.g., rheology or PSD) to the actual waste.

Two subclasses of chemical simulants exist. The first is called a "mixed oxide" simulant where particles matching the target chemistry are purchased from commercial vendors and mixed to the desired chemical composition. The second type is called "precipitated oxide" simulant where chemicals are dissolved in an aqueous solution and precipitated to form a slurry in a manner similar to the original waste processes.

Mixed-oxide simulants are less expensive and easier to produce than precipitated oxide simulants. However, both suffer from some serious drawbacks. The chemical composition is the key control variable, and particle morphology and PSD often vary from batch to batch. These subtle differences result in significant interfacial chemistry differences from the actual waste.

Consequently, differences exist between the colloid variables that control slurry rheology, such as zeta potential, inverse Debye length, and Hamaker constant. Often, the resulting fluid rheology from these simulant slurries does not match the actual waste measurements. Rheological modifiers have been used to overcome these differences and help match the chemical simulant rheology to the actual waste rheology. Due to these complexities, matching both physical and chemical properties of simulants is a difficult and time-consuming process. 
For determining critical velocity, chemical effects are of secondary importance. As shown in Figure 4.4, the inputs for determining critical velocity include particle size, particle density, fluid density, solids concentration, and slurry rheology. The interfacial chemistry affects slurry viscosity, which is an input to the critical-velocity design equations. In this manner, slurry chemistry only affects critical-velocity calculations indirectly.

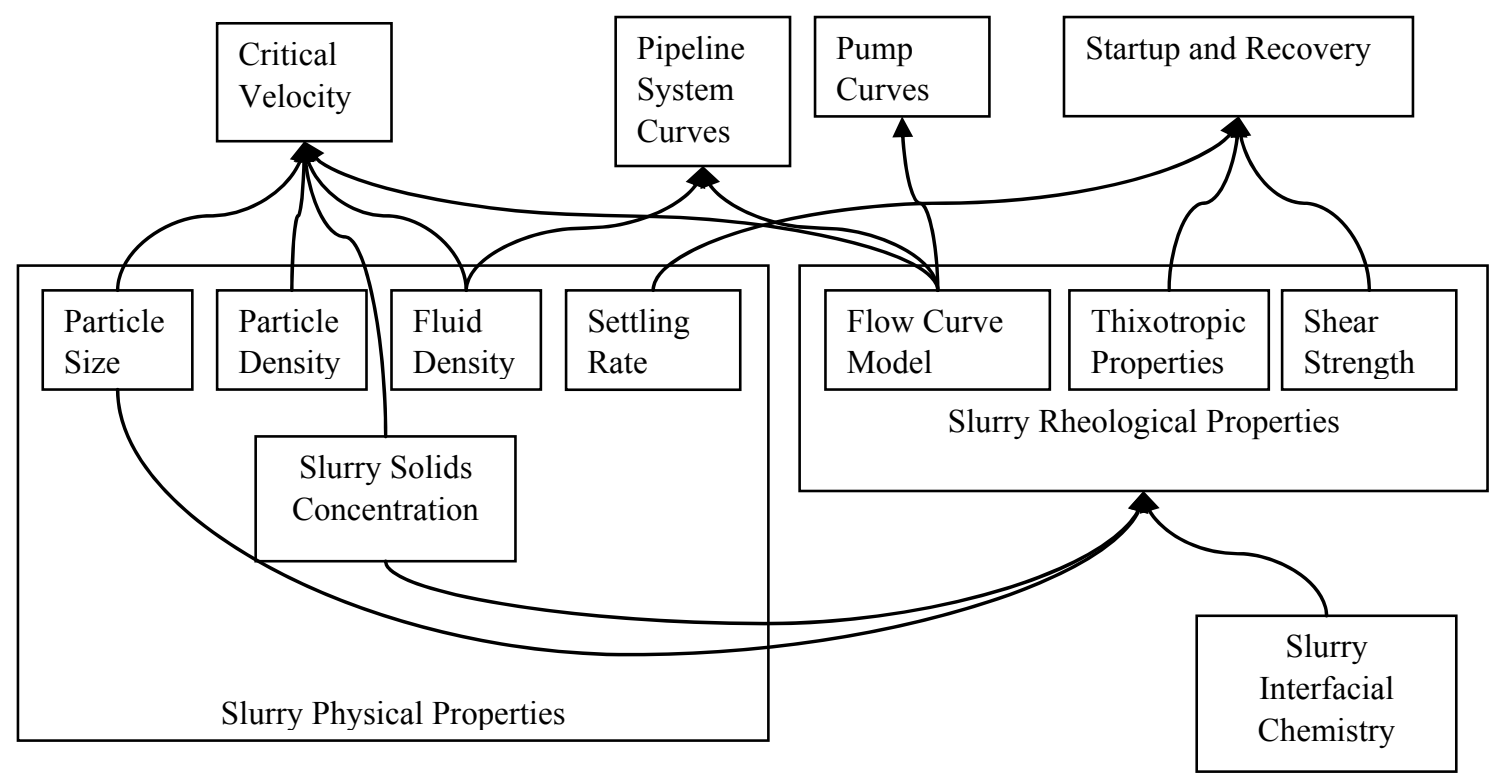

Figure 4.4. Slurry Property Inputs for Significant Pipeline Transport Calculations

One may argue that interfacial chemistry can also influence flocculation and agglomeration, which influence the particle size and density inputs to critical velocity. Thomas (1979) studied flocculated systems and developed the following conclusion:

When flocculation tendencies are present [18; the Thomas (1979) equation] will over-predict the deposit velocity because of the lower density of the flocs compared to the discrete particles. Thomas (1962) has previously considered such slurries and used the floc size and density measured in static settling tests to develop a deposition criterion. However, it was shown that by using a floc size and density closer to the values for a discrete particle his results are consistent with the present theory. This suggests that the floc size obtained under quiescent conditions is much larger than that present in turbulent pipe flow, and should not be used to characterize deposition from such flows.

Accordingly, for this work, discrete particles rather than flocs are used as the basis for simulant design and data analysis. Additionally, a simple physical simulant approach is used that targets a set criterion of discrete particle density, discrete particle size, and slurry rheological properties.

\subsection{Simulant Composition and Physical Properties}

Glass, alumina, and 316 stainless steel were selected as the coarse particle fraction in the experimental matrix. These materials have a nominal density of $2.5 \mathrm{~g} / \mathrm{cc}, 4.0 \mathrm{~g} / \mathrm{cc}$, and $8.0 \mathrm{~g} / \mathrm{cc}$ and satisfy the "Low," "Medium," and "High" particle density selections, respectively, that were proposed in 
Section 4.1. For stainless steel and glass, $10-\mu \mathrm{m}$ and $100-\mu \mathrm{m}$ particle powders were purchased to satisfy the "Low" and "High" size conditions outlined in Section 4.2. A 50- $\mu \mathrm{m}$ alumina powder was purchased to satisfy the "Middle" size selection. A suspension of these materials in water was used as the baseline (i.e., "low") rheological condition. A water and kaolin clay (Feldspar EPK) slurry was used to adjust the test rheology to the "Medium" and "High" conditions. The kaolin clay formed the fine particle fraction of the test mixture. Manufacturer and product information for each of the components is shown in Table 4.3. Throughout this report, references to $10-\mu \mathrm{m}, 50-\mu \mathrm{m}$, and $100-\mu \mathrm{m}$ sized materials refer to the nominal target values specified in Table 4.3. The measured particle size distributions for these materials are presented later in this section.

Table 4.3. Test Matrix and Slurry Materials Selected for Critical-Velocity Testing

\begin{tabular}{|c|c|c|c|c|}
\hline Particle Size $\rightarrow$ & Low & Medium & High & Fines \\
\hline Density $\downarrow$ & Nominal: $10 \mu \mathrm{m}$ & Nominal: $50 \mu \mathrm{m}$ & Nominal: $100 \mu \mathrm{m}$ & Nominal: $1 \mu \mathrm{m} \quad 2-3 \mathrm{~g} / \mathrm{cm}^{3}$ \\
\hline \multirow[t]{8}{*}{$\begin{array}{l}\text { Low } \\
2 \text { to } 4 \mathrm{~g} / \mathrm{cm}^{3}\end{array}$} & $\begin{array}{l}\text { Material: Soda } \\
\text { Glass }\end{array}$ & & Material: Soda Glass & \multirow{9}{*}{$\begin{array}{l}\text { Low: } \\
\text { Tap Water }\end{array}$} \\
\hline & & & Manufacturer: & \\
\hline & Manufacturer: & & Potters Industry & \\
\hline & Potters Industry & & & \\
\hline & & & Product: & \\
\hline & Product: & & Spheriglass $₫$ & \\
\hline & Spheriglass ${ }^{\circledR}$ & & A-glass 2227 & \\
\hline & A-glass 5000 & & & \\
\hline \multirow{6}{*}{$\begin{array}{l}\text { Medium } \\
4 \text { to } 6 \mathrm{~g} / \mathrm{cm}^{3}\end{array}$} & & Material: & & \\
\hline & & Aluminum Oxide & & \\
\hline & & $\begin{array}{l}\text { Manufacturer: } \\
\text { Washington Mills }\end{array}$ & & $\begin{array}{l}\text { Medium/High: } \\
\text { Slurries of Kaolin Clay } \\
\text { in Water }\end{array}$ \\
\hline & & Product: & & \multirow{2}{*}{$\begin{array}{l}\text { Manufacturer: } \\
\text { Feldspar Corp. } \\
\text { Product: } \\
\text { EPK Kaolin }\end{array}$} \\
\hline & & Duralum $\AA$ & & \\
\hline & & 220 grit & & \multirow{8}{*}{$\begin{array}{l}\text { Product: } \\
\text { EPK Kaolin }\end{array}$} \\
\hline \multirow{7}{*}{$\begin{array}{l}\text { High } \\
8 \text { to } 10 \mathrm{~g} / \mathrm{cm}^{3}\end{array}$} & Material: & & Material: & \\
\hline & 316 Stainless & & 316 Stainless Steel & \\
\hline & & & Manufacturer: & \\
\hline & Manufacturer: & & AMETEK & \\
\hline & & & Product: & \\
\hline & Product: & & P316L & \\
\hline & $\begin{array}{l}\text { P316L } \\
(10 \mathrm{M})\end{array}$ & & $(-100+170)$ & \\
\hline
\end{tabular}

Shaded cells are included in reduced factorial design.

The physical properties of the resulting test slurries are documented in Table 4.4 to Table 4.6 for the low, medium, and high rheology cases, respectively. For each test, the bulk density of the slurry was measured in situ with Coriolis flow meters while the flow loop and agitation system were operating. The mass of coarse particles was measured while preparing the test simulant. Additionally, the total volume in the system was measured via level probes in the mixing vessel. These data allow one to calculate the 
theoretical volume fraction of coarse particles in the flow loop system. Coupled with the Coriolis meter bulk density, the density of the interstitial fluid (i.e., water or kaolin/water slurry) can be calculated. From the interstitial fluid density, the fraction of fines (i.e., kaolin clay) and water can be calculated. With this information the density and compositional data for the coarse, fine, and water fractions can be calculated. Particle densities were taken from data provided by the manufacturer. These values were substantiated by separate laboratory measurements on test samples.

In the case of the 100- $\mu$ m stainless steel systems (i.e., HHM and HHH), the particles settle quickly enough that the flow loop density was about $30 \%$ below the theoretical value calculated by the simulant mass and volume calculations. For these samples, the interstitial fluid density (i.e., kaolin and water slurry) was determined to be approximately $1.10 \mathrm{~g} / \mathrm{mL}$ from separate water-content measurements on test samples.

The PSD of each of the simulants was measured using laser diffraction technology. A Microtrac S3000 Particle Size Analyzer was used for these measurements. Software for the particle size analyzer calculates the PSD from the light-scattering patterns using Mie scattering theory. Particles with diameters between 0.02 and $1400 \mu \mathrm{m}$ were analyzed to determine the PSD of the simulants. A particle-size standard traceable to the National Institute of Standards and Technology (NIST) was measured before measuring the distribution of these simulants.

Small aliquots of the simulant samples $(<1 \mathrm{~mL})$ were diluted in water in a variable speed recirculator (Microtrac VSR) before making the particle-size measurements. The total volume of the recirculator is $300 \mathrm{~mL}$. Appropriate dilutions were determined by the amount of light passing through the diluted material (obscuration), which was measured by the particle-size analyzer. Measurements were made with circulation rates of $80 \%$ and $45 \%$ of the maximum circulation rate of $90 \mathrm{~mL} / \mathrm{sec}$. Higher circulation rates ( $80 \%$ of the maximum) provided the most accurate results; therefore, all of the data reported are at circulation rates of $80 \%$.

All of the simulant samples were shaken before taking aliquots for PSD measurements. Measurements were made on samples with no further treatment before dilution and on samples that were sonicated before dilution. Sonication was performed with a Microson ultrasonic cell disruptor with a microtip probe. Twenty short pulses $(<10$ seconds) were used to de-agglomerate and/or disperse the particles in the aliquot. To improve sonication the samples were first diluted by approximately a factor of 10. A polymer deflocculant (Darvan $821 \mathrm{~A}$ ) was used with the simulants containing alumina to improve dispersion of the alumina particles during PSD measurements. Particle size data reported for simulants containing stainless steel and glass beads as the coarse particles are for the sonicated aliquots. Particlesize data reported for simulants containing alumina were obtained from sonicated aliquots with deflocculant added.

Using the protocol described in this paragraph, the same batch of coarse particles was used for each rheological test condition. The progression of testing was to perform the "low" rheology tests first. After testing the supernatant liquid was decanted from the coarse particles, and kaolin clay/water slurry was added. After kaolin slurry was added, a sample was drawn, and a flow curve was measured. If the Bingham model fit yield stress was below the "high" rheology target of 6-Pa Bingham yield stress, the yield stress was increased by either removing water via settle/decant or by adding $\mathrm{MgSO}_{4}$. On the order of 10 parts per million of $\mathrm{MgSO}_{4}$ were required to significantly raise the yield stress of the slurry. For 
this reason, the rheological properties sometimes do not follow the same trend as solids concentration. If the Bingham yield stress was too high, tap water was added to lower the yield stress of the test slurry. This process was then repeated for the "high" rheology case.

The flow curve was obtained on a rheometer configured with concentric-cylinder geometry. The shear rate was ramped from 0 to $1000 \mathrm{~s}^{-1}$ over a 5 -minute period (Smith and Prindiville 2002). The shear rate was held at $1000 \mathrm{~s}^{-1}$ for 1 minute and then ramped down from 1000 to $0 \mathrm{~s}^{-1}$ over another 5 -minute period. Bingham plastic and Casson curve fits were obtained for the up and down ramp portions of the curve over a typical range of 0 to $600 \mathrm{~s}^{-1}$ for the "Medium" and "High" rheology samples. A $100-\mathrm{s}^{-1}$ upper limit was used for the "Low" rheology samples. These upper limits were established because of Taylor vortex formation at higher rotational rates. Note that the distance between the bottom of the rheometer cup and bob was adjusted to allow for sedimentation of coarse particles to occur. Otherwise, the rheometer bob would have increased drag from being partially submerged in a sediment bed. The increased drag results in inaccurate flow curve measurements. For this reason, the viscosity of the carrier fluid, water and kaolin clay slurry, could only be measured. 
Table 4.4. Properties of "Low" Rheology Simulant Test Matrix

\begin{tabular}{|c|c|c|c|c|c|c|}
\hline Test Configuration & & 1 & 2 & 3 & 4 & 5 \\
\hline Acronym & & LLL & HLL & MML & LHL & HHL \\
\hline \multicolumn{7}{|c|}{ Mass per Unit Volume (g/L) } \\
\hline Coarse Particles & & 245 & 741 & 327 & 185 & 236 \\
\hline Fine Particles & & 0 & 0 & 0 & 0 & 0 \\
\hline Water & & 901 & 906 & 912 & 925 & 969 \\
\hline \multicolumn{7}{|c|}{ Volume Fraction (vol\%) } \\
\hline Coarse Particles & & $9.8 \%$ & $9.3 \%$ & $8.7 \%$ & $7.4 \%$ & $3.0 \%$ \\
\hline Fine Particles & & $0.0 \%$ & $0.0 \%$ & $0.0 \%$ & $0.0 \%$ & $0.0 \%$ \\
\hline Water & & $90.2 \%$ & $90.7 \%$ & $91.3 \%$ & $92.6 \%$ & $97.0 \%$ \\
\hline \multicolumn{7}{|c|}{ Mass Fraction (mass\%) } \\
\hline Coarse Particles & & $21.4 \%$ & $45.0 \%$ & $26.4 \%$ & $16.6 \%$ & $19.6 \%$ \\
\hline Fine Particles & & $0.0 \%$ & $0.0 \%$ & $0.0 \%$ & $0.0 \%$ & $0.0 \%$ \\
\hline Water & & $78.6 \%$ & $55.0 \%$ & $73.6 \%$ & $83.4 \%$ & $80.4 \%$ \\
\hline \multicolumn{7}{|c|}{ Component Density (kg/L) } \\
\hline Coarse Particles & & 2.50 & 7.95 & 3.77 & 2.50 & 7.95 \\
\hline Fine Particles & & 2.50 & 2.50 & 2.50 & 2.50 & 2.50 \\
\hline Water & & 1.00 & 1.00 & 1.00 & 1.00 & 1.00 \\
\hline Bulk & & 1.15 & 1.65 & 1.24 & 1.11 & 1.21 \\
\hline \multicolumn{7}{|c|}{ Particle Size Distribution $(\mu \mathrm{m})$} \\
\hline $\mathrm{d}_{5}$ & & 1.6 & 7.2 & 30.9 & 91.0 & 55.2 \\
\hline$d_{10}$ & & 2.8 & 8.4 & 38.2 & 97.4 & 68.1 \\
\hline$d_{20}$ & & 3.8 & 9.8 & 49.7 & 106.4 & 90.8 \\
\hline$d_{30}$ & & 4.6 & 11.2 & 56.7 & 113.1 & 112.0 \\
\hline $\mathrm{d}_{40}$ & & 5.4 & 13.1 & 62.4 & 119.4 & 127.9 \\
\hline $\mathrm{d}_{50}$ & & 6.2 & 15.1 & 67.6 & 125.9 & 140.3 \\
\hline $\mathrm{d}_{60}$ & & 7.2 & 17.3 & 73.2 & 132.8 & 151.3 \\
\hline $\mathrm{d}_{70}$ & & 8.9 & 20.4 & 79.9 & 140.6 & 161.9 \\
\hline $\mathrm{d}_{80}$ & & 10.7 & 24.7 & 89.0 & 150.7 & 173.8 \\
\hline $\mathrm{d}_{90}$ & & 12.5 & 31.4 & 107.3 & 167.0 & 190.6 \\
\hline $\mathrm{d}_{95}$ & & 14.4 & 37.7 & 129.5 & 182.3 & 203.9 \\
\hline \multicolumn{7}{|c|}{ Flow Curve $\left(0-100 \mathrm{~s}^{-1}\right)$} \\
\hline Newtonian Viscosity ${ }^{(a)}$ & $\mathrm{cP}$ & 1.0 & 1.0 & 1.0 & 1.0 & 1.0 \\
\hline Standard Error & & 25.5 & 26.8 & 19.1 & 33.6 & $0.9776^{(\mathrm{b})}$ \\
\hline
\end{tabular}

(a) Note that due to sedimentation in the rheometer it was possible to only measure the viscosity of the carrier fluid, water.

(b) Error reported for these model fits is $r^{2}$ value. 
Table 4.5. Properties of "Medium" Rheology Simulant Test Matrix

\begin{tabular}{|c|c|c|c|c|c|c|c|c|}
\hline \multicolumn{2}{|c|}{ Test Configuration } & 6 & 7 & $\begin{array}{c}8 \\
\text { (run 1) }\end{array}$ & $\begin{array}{c}8 \\
\text { (run 2) }\end{array}$ & $\begin{array}{c}8 \\
\text { (run 3) }\end{array}$ & 9 & 10 \\
\hline \multicolumn{2}{|l|}{ Acronym } & LLM & HLM & MMM & MMM & MMM & LHM & HHM \\
\hline \multicolumn{9}{|c|}{ Mass per Unit Volume (g/L) } \\
\hline \multicolumn{2}{|l|}{ Coarse Particles } & 243 & 755 & 357 & 358 & 357 & 210 & 313 \\
\hline \multicolumn{2}{|l|}{ Fine Particles } & 307 & 175 & 223 & 223 & 223 & 192 & 162 \\
\hline \multicolumn{2}{|l|}{ Water } & 779 & 834 & 815 & 815 & 815 & 838 & 895 \\
\hline \multicolumn{9}{|c|}{ Volume Fraction (vol\%) } \\
\hline \multicolumn{2}{|l|}{ Coarse Particles } & $9.7 \%$ & $9.5 \%$ & $9.5 \%$ & $9.5 \%$ & $9.5 \%$ & $8.4 \%$ & $3.9 \%$ \\
\hline \multicolumn{2}{|l|}{ Fine Particles } & $12.3 \%$ & $7.0 \%$ & $8.9 \%$ & $8.9 \%$ & $8.9 \%$ & $7.7 \%$ & $6.5 \%$ \\
\hline \multicolumn{2}{|l|}{ Water } & $78.0 \%$ & $83.5 \%$ & $81.6 \%$ & $81.6 \%$ & $81.6 \%$ & $83.9 \%$ & $89.6 \%$ \\
\hline \multicolumn{9}{|c|}{ Mass Fraction (mass\%) } \\
\hline \multicolumn{2}{|l|}{ Coarse Particles } & $18.3 \%$ & $42.8 \%$ & $25.6 \%$ & $25.6 \%$ & $25.6 \%$ & $16.9 \%$ & $22.9 \%$ \\
\hline \multicolumn{2}{|l|}{ Fine Particles } & $23.1 \%$ & $9.9 \%$ & $16.0 \%$ & $16.0 \%$ & $16.0 \%$ & $15.5 \%$ & $11.8 \%$ \\
\hline \multicolumn{2}{|l|}{ Water } & $58.6 \%$ & $47.3 \%$ & $58.4 \%$ & $58.4 \%$ & $58.4 \%$ & $67.6 \%$ & $65.3 \%$ \\
\hline \multicolumn{9}{|c|}{ Component Density $(\mathrm{kg} / \mathrm{L})$} \\
\hline \multicolumn{2}{|l|}{ Coarse Particles } & 2.50 & 7.95 & 3.77 & 3.77 & 3.77 & 2.50 & 7.95 \\
\hline \multicolumn{2}{|l|}{ Fine Particles } & 2.50 & 2.50 & 2.50 & 2.50 & 2.50 & 2.50 & 2.50 \\
\hline \multicolumn{2}{|l|}{ Water } & 1.00 & 1.00 & 1.00 & 1.00 & 1.00 & 1.00 & 1.00 \\
\hline \multicolumn{2}{|l|}{ Bulk } & 1.33 & 1.76 & 1.40 & 1.40 & 1.40 & 1.24 & 1.37 \\
\hline \multicolumn{9}{|c|}{ Particle Size Distribution $(\mu \mathrm{m})$} \\
\hline \multicolumn{2}{|l|}{$\mathrm{d}_{5}$} & 0.7 & 0.7 & 3.5 & 3.3 & 11.2 & 0.7 & 2.3 \\
\hline \multicolumn{2}{|l|}{$d_{10}$} & 1.2 & 2.9 & 7.1 & 6.7 & 24.5 & 0.9 & 5.2 \\
\hline \multicolumn{2}{|l|}{$d_{20}$} & 3.2 & 4.6 & 23.3 & 24.7 & 44.4 & 4.1 & 28.2 \\
\hline \multicolumn{2}{|l|}{$d_{30}$} & 4.2 & 6.1 & 43.7 & 42.4 & 54.6 & 6.8 & 98.6 \\
\hline \multicolumn{2}{|l|}{$d_{40}$} & 5.2 & 8.1 & 53.7 & 51.6 & 62.5 & 11.3 & 117.8 \\
\hline $\mathrm{d}_{50}$ & & 6.2 & 10.5 & 62.2 & 62.4 & 69.5 & 18.5 & 130.4 \\
\hline$d_{60}$ & & 8.1 & 12.4 & 70.4 & 73.8 & 76.3 & 87.6 & 141.2 \\
\hline $\mathrm{d}_{70}$ & & 10.9 & 15.1 & 79.0 & 81.9 & 83.3 & 106.0 & 152.6 \\
\hline $\mathrm{d}_{80}$ & & 13.9 & 19.3 & 88.7 & 89.3 & 91.4 & 116.4 & 165.5 \\
\hline $\mathrm{d}_{90}$ & & 23.4 & 26.7 & 101.0 & 96.9 & 102.3 & 129.3 & 184.1 \\
\hline $\mathrm{d}_{95}$ & & 45.1 & 36.8 & 110.9 & 101.9 & 111.3 & 140.9 & 201.6 \\
\hline & & Bingha & Flow $\mathrm{Cu}$ & e $(0-600 \mathrm{~s}$ & & & & \\
\hline Bingham Yield Stress & $\mathrm{Pa}$ & 2.8 & 3.0 & 3.5 & 3.5 & 3.5 & 3.4 & 3.2 \\
\hline Bingham Consistency & $\mathrm{cP}$ & 6.8 & 7.3 & 6.9 & 6.9 & 6.9 & 5.5 & 4.3 \\
\hline Standard Error & & 16.0 & 16.0 & 22.1 & 22.1 & 22.1 & 24.6 & 34.7 \\
\hline & & Casso & Flow Cur & $\left(0-600 \mathrm{~s}^{-}\right.$ & & & & \\
\hline Casson Yield Stress & $\mathrm{Pa}$ & 1.7 & 2.0 & 2.4 & 2.4 & 2.4 & 2.4 & 2.2 \\
\hline Infinite Shear Viscosity & $\mathrm{cP}$ & 2.7 & 2.5 & 2.3 & 2.3 & 2.3 & 1.8 & 1.4 \\
\hline Standard Error & & 9.2 & 8.2 & 9.2 & 9.2 & 9.2 & 8.3 & 14.6 \\
\hline
\end{tabular}

(a) Note that due to sedimentation in the rheometer it was possible to only measure the viscosity of the carrier fluid, kaolin clay slurry. 
Table 4.6. Properties of "High" Rheology Simulant Test Matrix

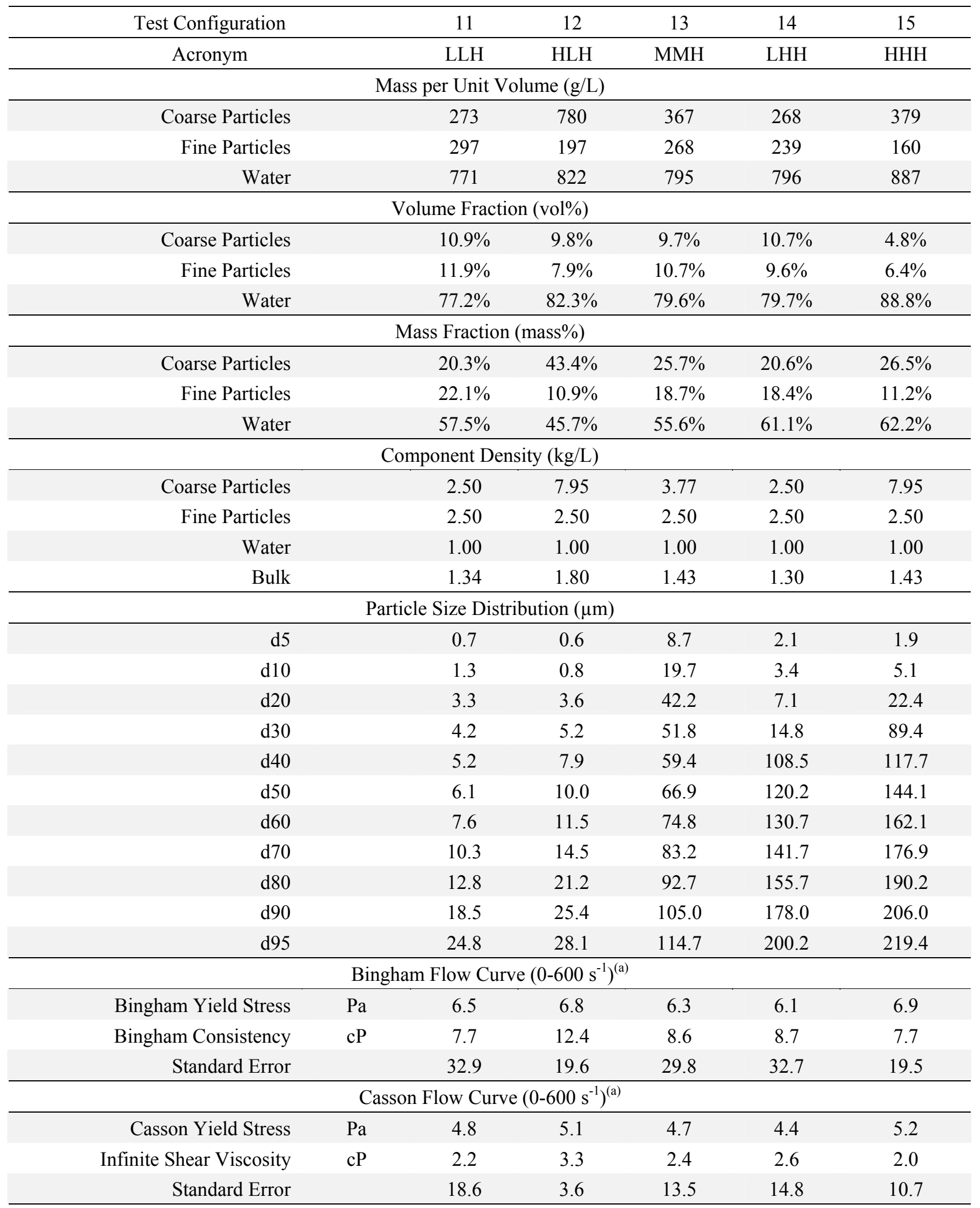

(a) Note that due to sedimentation in the rheometer it was possible to only measure the viscosity of the carrier fluid, kaolin clay slurry. 
Scanning electron microscope images of the coarse particle components are shown in Figure 4.5 to Figure 4.9. In terms of particle shape, the glass-bead media are spherical, the alumina media is fractured and angular, and the stainless steel media are irregular spheroids.

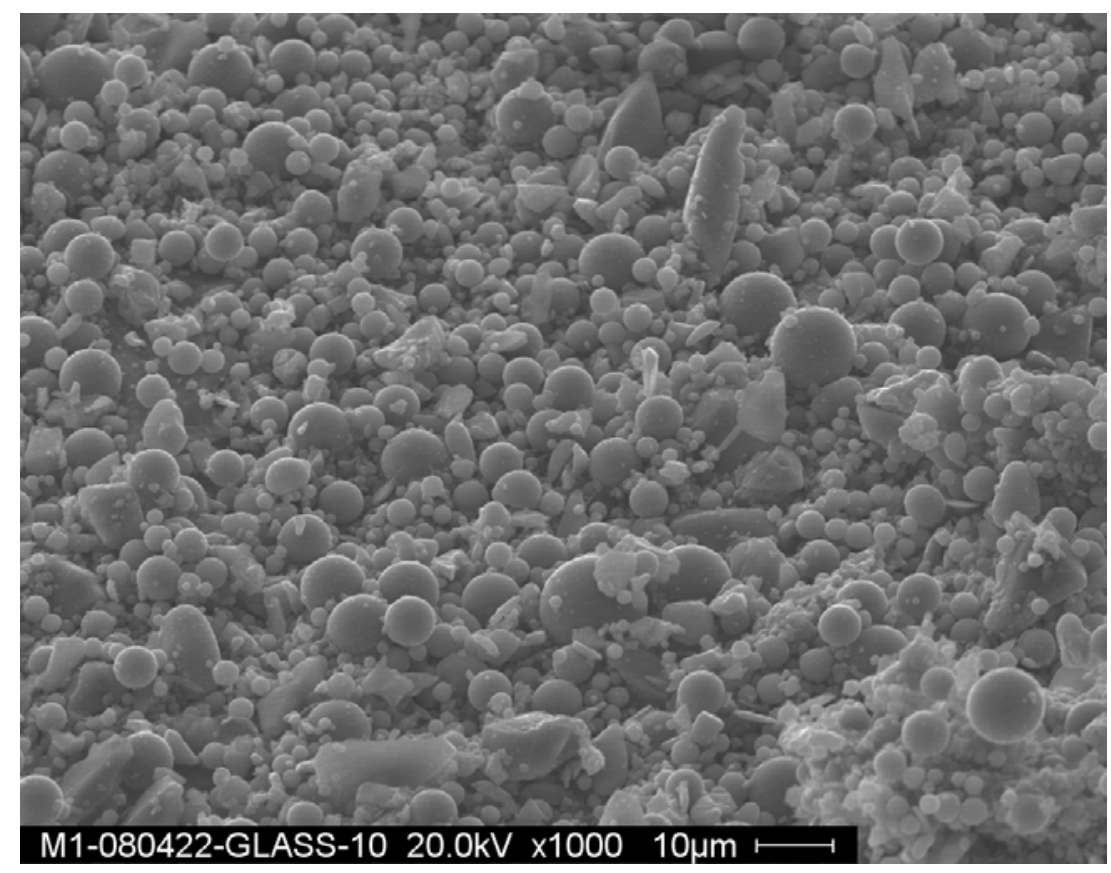

Figure 4.5. Micrograph of LL Coarse Particles (10- $\mu$ m glass beads; Potters Industry Spheriglass ${ }^{\circledR}$ A-glass 5000)

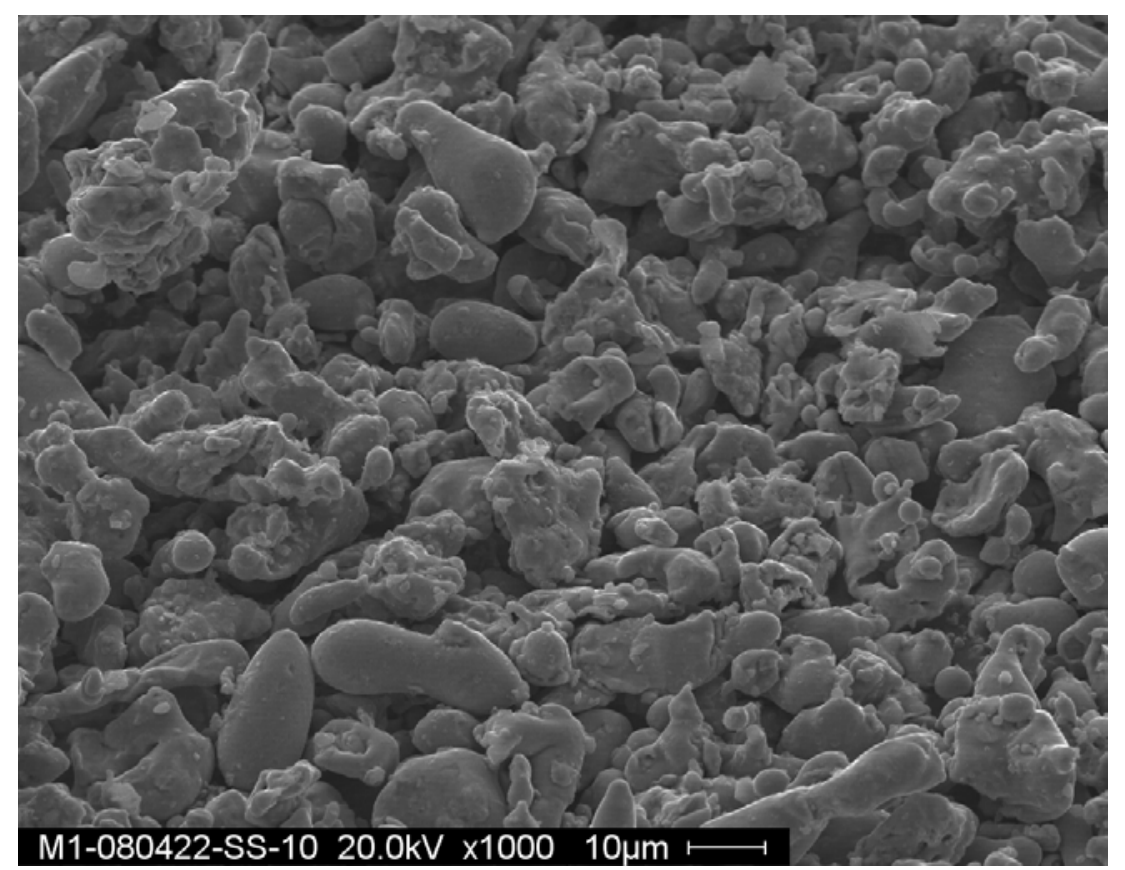

Figure 4.6. Micrograph of HL Coarse Particles (10- $\mu$ m stainless steel; AMETEK P316L $10 \mathrm{M})$ 


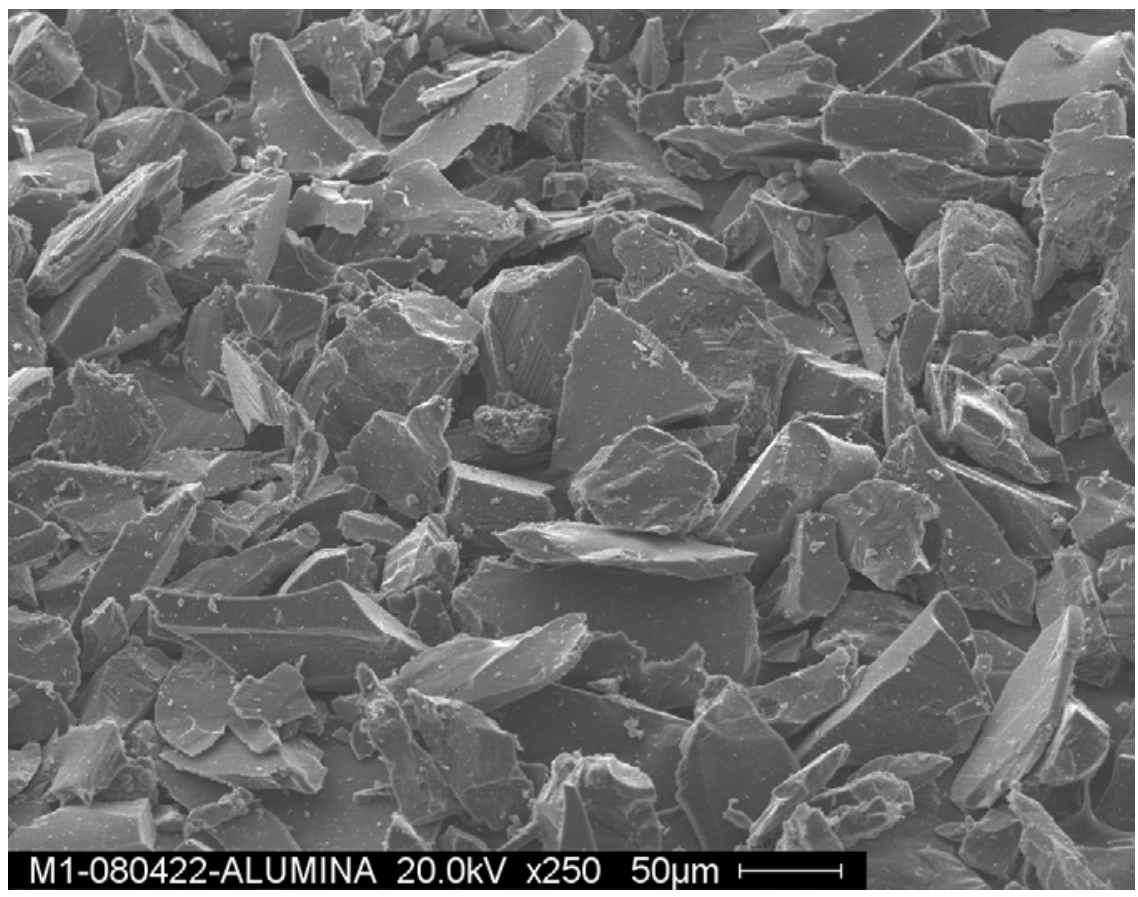

Figure 4.7. Micrograph of MM Coarse Particles (50- $\mu \mathrm{m}$ Alumina; Washington Mills Duralum ${ }^{\circledR}$ 220 grit)

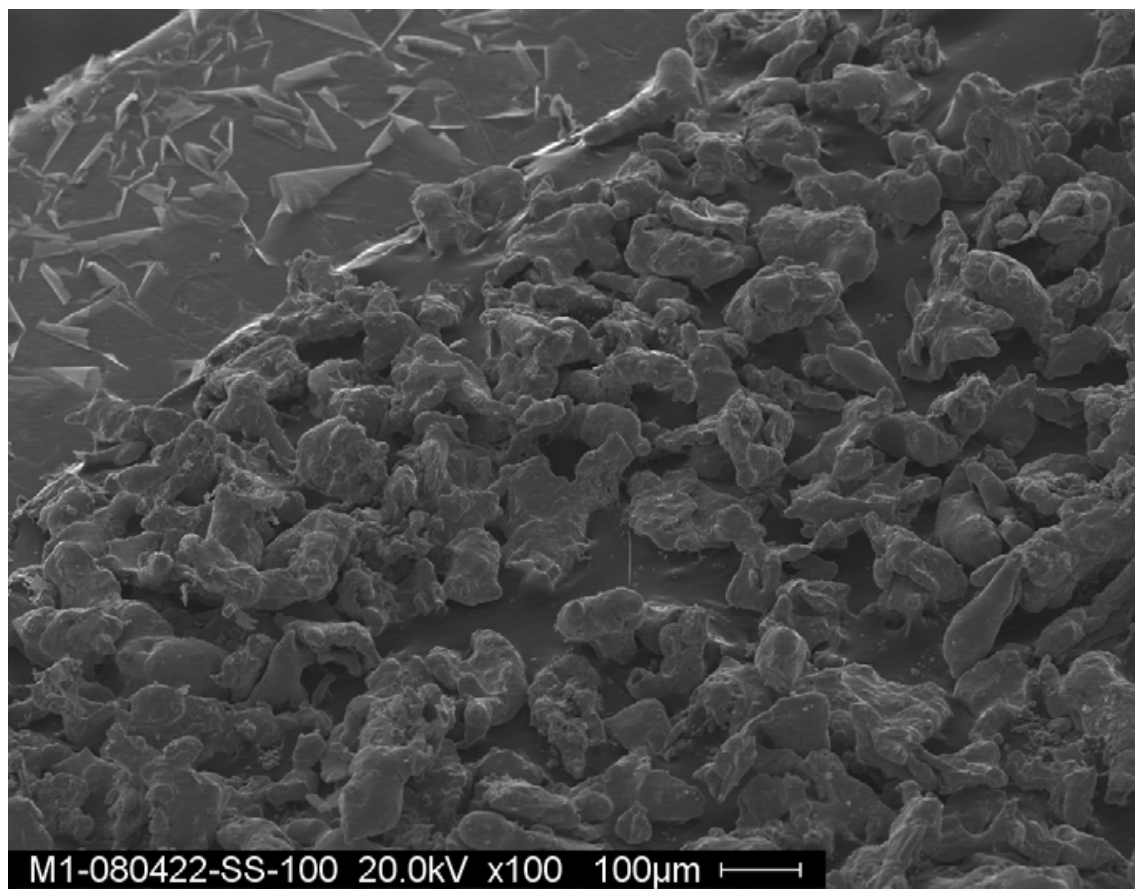

Figure 4.8. Micrograph of HH Coarse Particles (100- $\mu \mathrm{m}$ stainless steel; AMETEK P316L -100+170) 


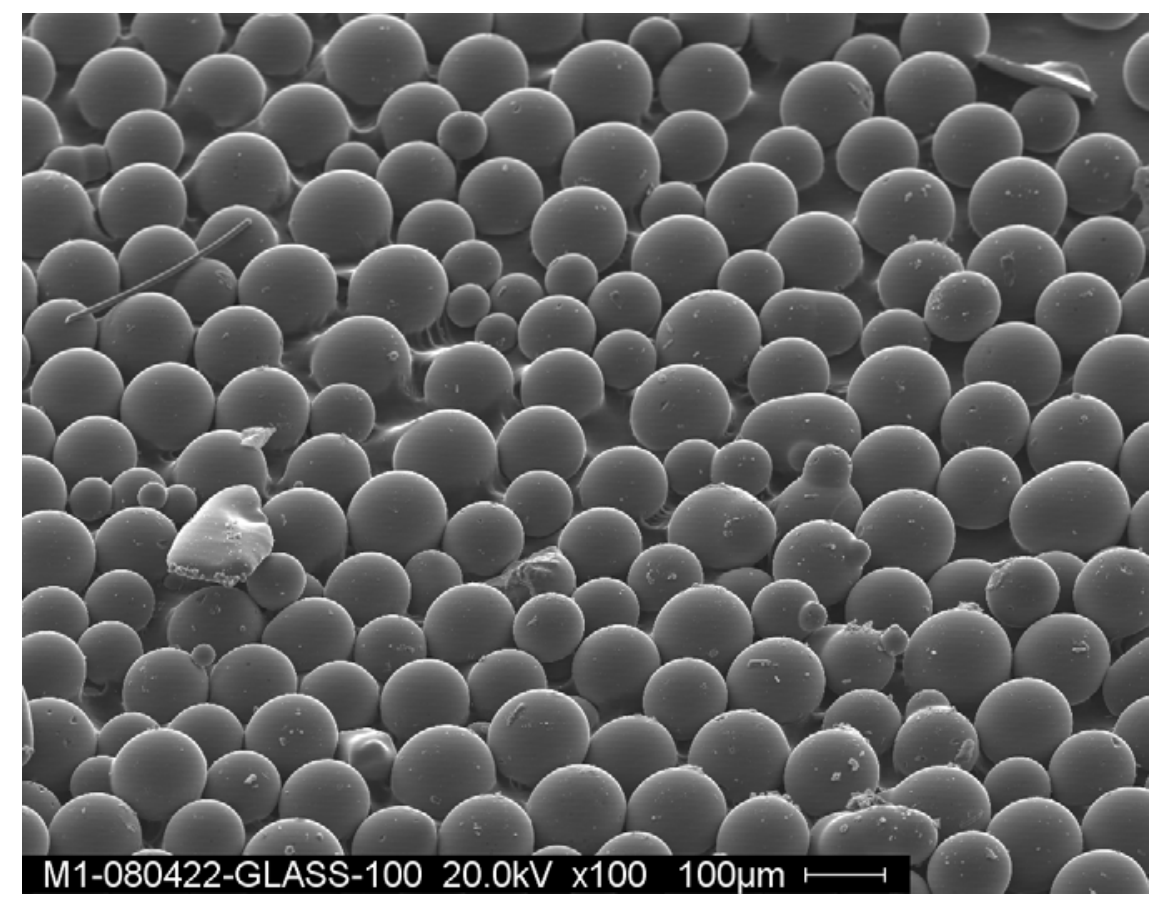

Figure 4.9. Micrograph of LH Coarse Particles (100- $\mu$ m glass beads; Potters Industry Spheriglass ${ }^{\circledR}$ A-glass 2227) 



\subsection{Test Apparatus}

This section describes the flow-loop components and instrumentation. A schematic of the flow-loop system is shown in Figure 5.1. Before a test, a batch of simulant is placed in a slurry mixing tank. During operation, the slurry is transported around the flow loop through the slurry pump (P-1). The slurry then enters an inlet Coriolis flow meter (CM-1) and into the main leg of the flow loop. The pressure gradient of the flow in a straight horizontal pipe section is measured with a differential pressure transducer (DP Port \#1). The slurry then enters an electrical resistance tomography (ERT) probe where a cross-sectional image of slurry conductivity is obtained in real time. Next, a clear polyvinyl chloride (PVC) section of pipe is installed to visually inspect the state of the flow. A second differential pressure transducer (DP Port \#2) is located on the return leg of the flow loop for a redundant pressure-drop measurement. An outlet Coriolis flow meter (CM-2) again is used for redundant flow measurements. The distribution of the slurry particle chord length is then measured in real time with a Lasentec probe. Lastly, the total loop pressure is measured with a third differential pressure transducer (DP Port \#3). The slurry then returns back to the mixing tank, and the process is repeated. A chiller is connected to the mixing-tank water jacket to maintain constant temperature throughout the test.

At the conclusion of the test, the system is flushed by a pneumatically driven flush tank. A charge of flush water is loaded into the flush tank. The air compressor then charges the air receiver tank to the target flush pressure. Valves are then aligned to isolate the slurry mixing tank and allow a path from the flush tank, through the flow loop, and to a simulant capture tank. A description of these system components follows.

\subsection{Piping}

The piping used for the flow loop consists primarily of 3-inch schedule-40, 304-stainless steel (with some recycled 316-stainless steel pieces). The piping in the differential pressure port \#1 and \#2 sections comes from the WTP excess yard and meets the "SC-11" project specifications. A schematic of the major flow loop piping system with dimensions is shown in Figure 5.2. The distance between the differential pressure port legs is $18 \mathrm{ft} 8$ 3/4 in (224.75 in.) for both DP Port \#1 and DP Port \#2. The pressure transducer system is discussed in greater detail in Section 5.6. 



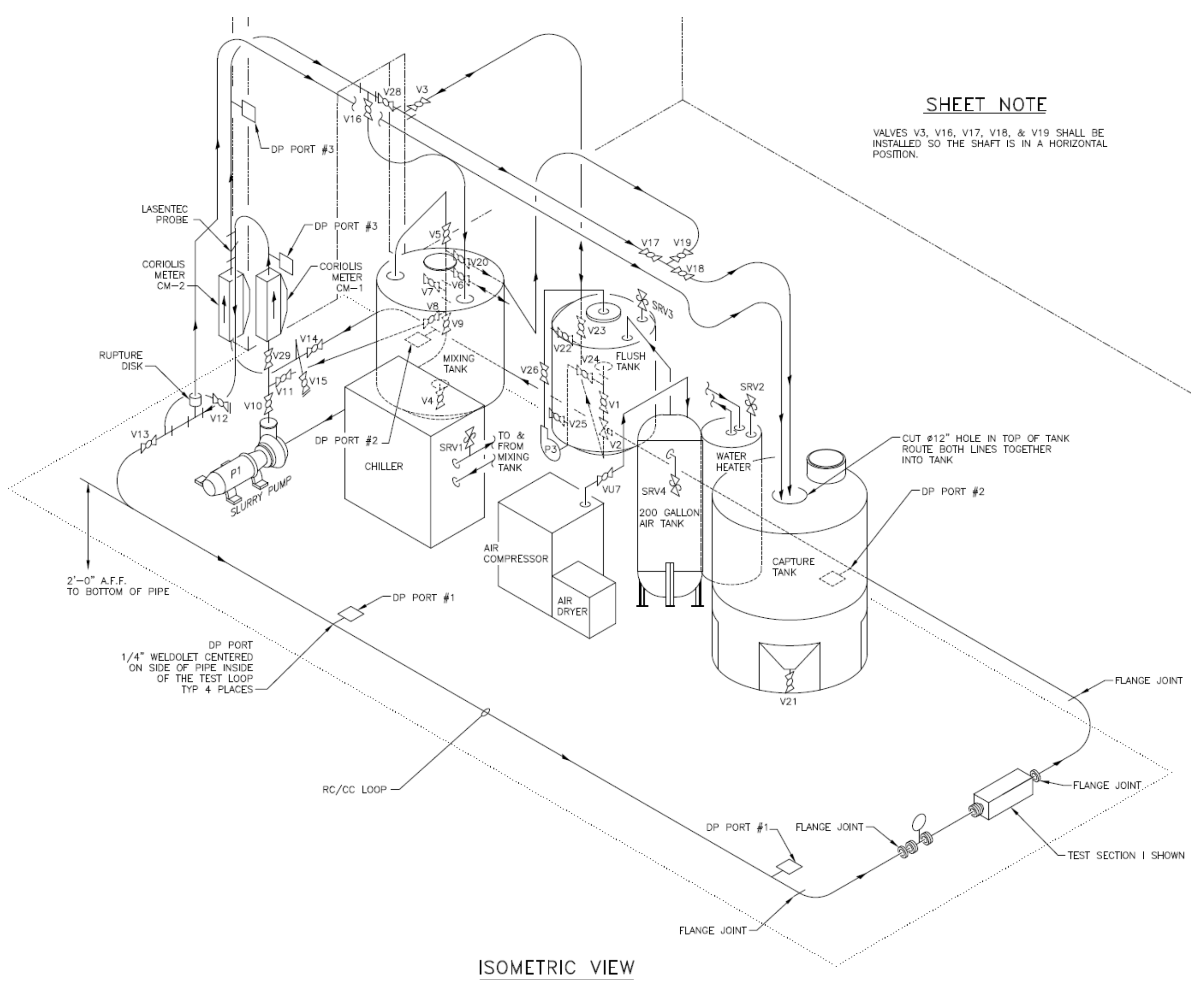

Figure 5.1. Schematic of Flow Loop System 



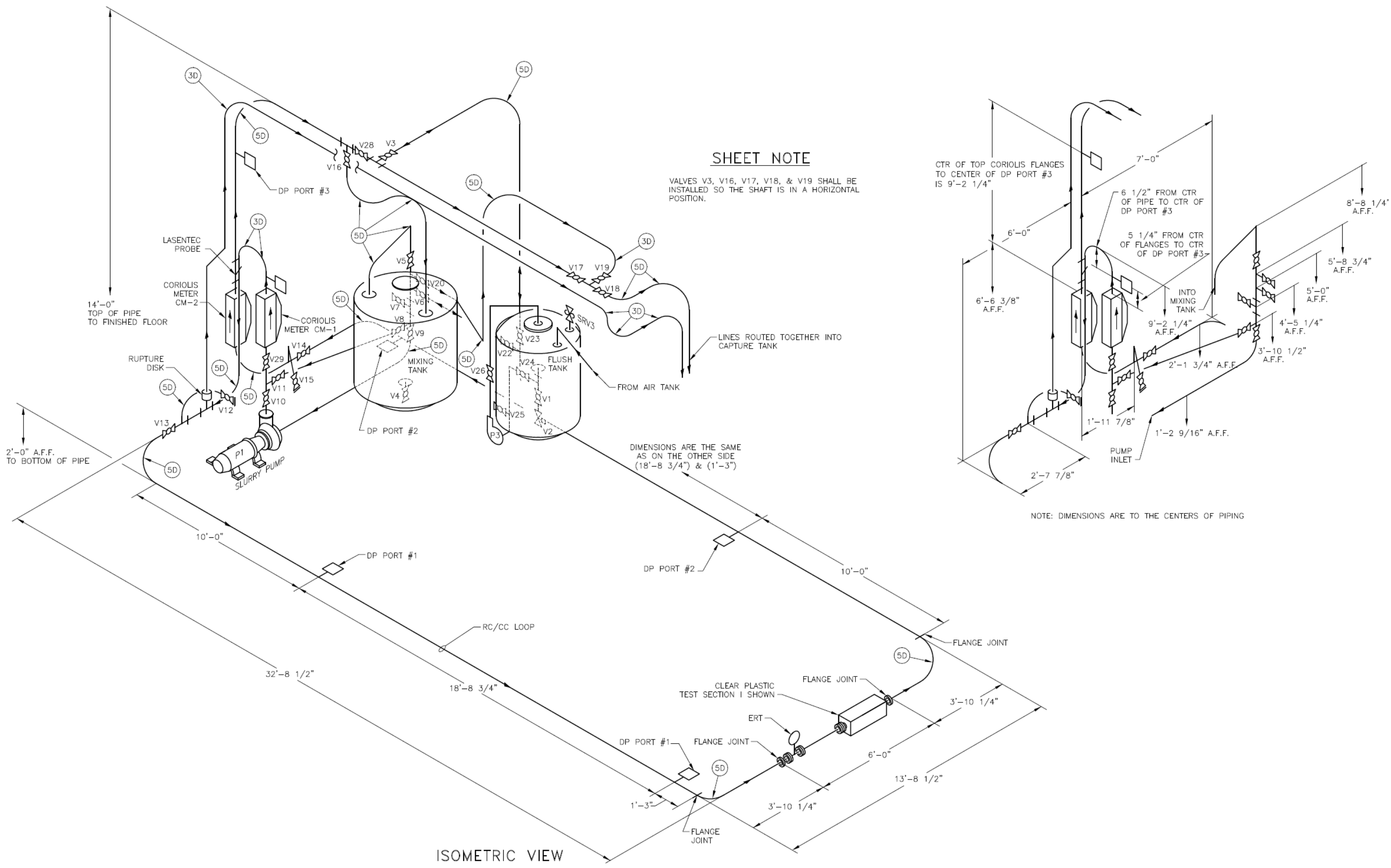

Figure 5.2. Schematic of Flow Loop Piping System with Major Dimensions 


\subsection{Slurry Pump}

The slurry pump was a Georgia Iron Works GIW 2X3LCC-M9 (LCC-M 50-230.2K M1). A picture of a similar pump is shown in Figure 5.3. The pump is driven by a 15-hp, 1800-rpm, totally enclosed fancooled 460-V electric motor produced by Reliance XEX as model P25G3316. This motor is connected to the pump by a belt drive. A Flowserve SL-C single cartridge flushless mechanical seal is used in this pump.

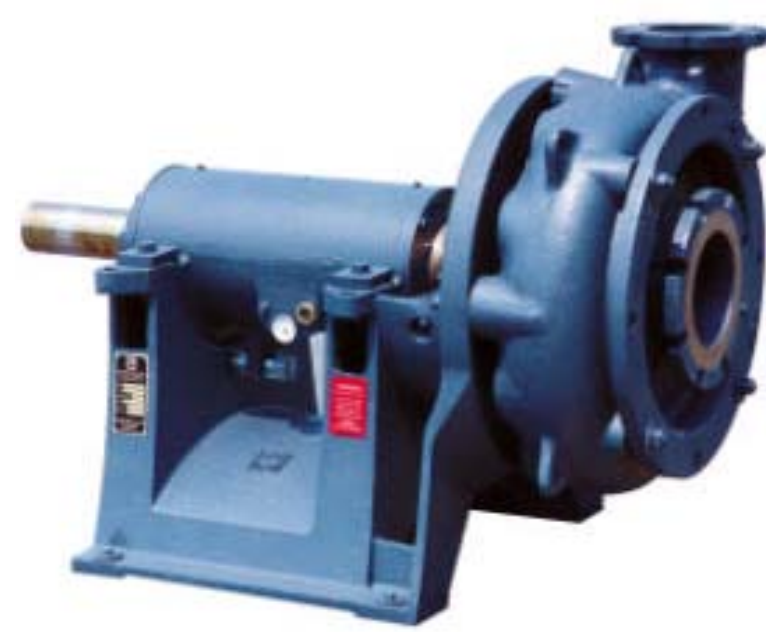

Figure 5.3. Photograph of Georgia Iron Works 2X3LCC Slurry Pump (source: www.giwindustries.com)

\subsection{Mixing Tank}

The mixing tank was a standard four-baffle mixing tank with a 54-inch internal diameter. The maximum capacity of this tank was approximately 400 gallons. The tank is agitated by a 25 inch diameter, 4-bladed pitched-blade impeller (4PBT45) driven by a 10-hp overhead electric motor manufactured by Philadelphia mixer. At full speed, the system rotates at $155 \mathrm{rpm}$. A dip-tube system is used to feed and return the slurry from the test loop. A drawing of the mixing tank internal components is shown in Figure 5.4.

\subsection{Flush System}

The flush tank was a "U" stamped pressure vessel (National Board number 18,365) rated to a maximum working pressure of $150 \mathrm{psig}$. The tank diameter is $4 \mathrm{ft}$ with a capacity of 400 gallons. The tank is jacketed to allow for temperature control. The flush tank was augmented with a jet mixer system. A drawing of the mixing tank's internal components is shown in Figure 5.5.

\subsection{Data-Acquisition System}

The data-acquisition system took temperature, flow, level, and pressure data from the flow loop and stored it in data files. The system was running National Instruments LabView ${ }^{\mathrm{TM}}$ software with a nominal sampling rate of $300 \mathrm{~ms}$. 


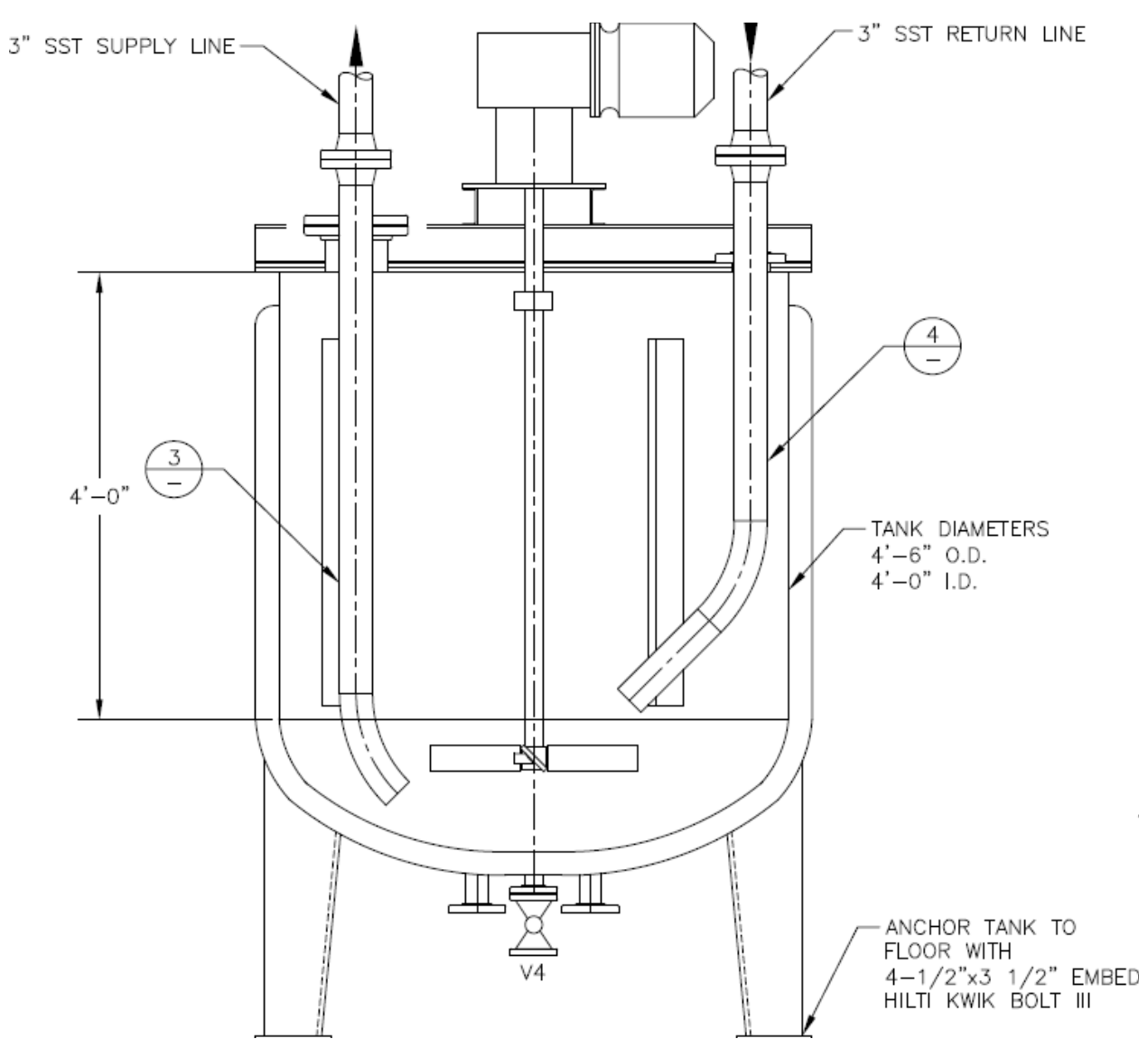

Figure 5.4. Schematic of Mixing Tank Internal Components

\subsection{Differential-Pressure Transducers}

Rosemount 1151 differential-pressure transducers were used to measure flow-loop pressure gradients. The pressure transducers were connected to the flow loop through open horizontal weldolet connectors. The open design of the ports was selected over diaphragm systems to allow for greater pressure sensitivity. Since sediment was expected to fill these ports during operation, a differential-pressure port purge system was also installed. This system allowed the differential-pressure transducers to be isolated from the flow loop. The differential-pressure ports were then cleaned by briefly flushing lines with highpressure water. This purge process was implemented as needed throughout testing. Two transducers with differing pressure measurement ranges were connected to a pair of weldolet ports. This allowed for a broad range of pressure measurements to be conducted.

\subsection{Coriolis Meters}

The Coriolis meters were Micro-Motion F-Series sensors designed for 3-inch schedule 40 pipe and made of 316L stainless steel. 


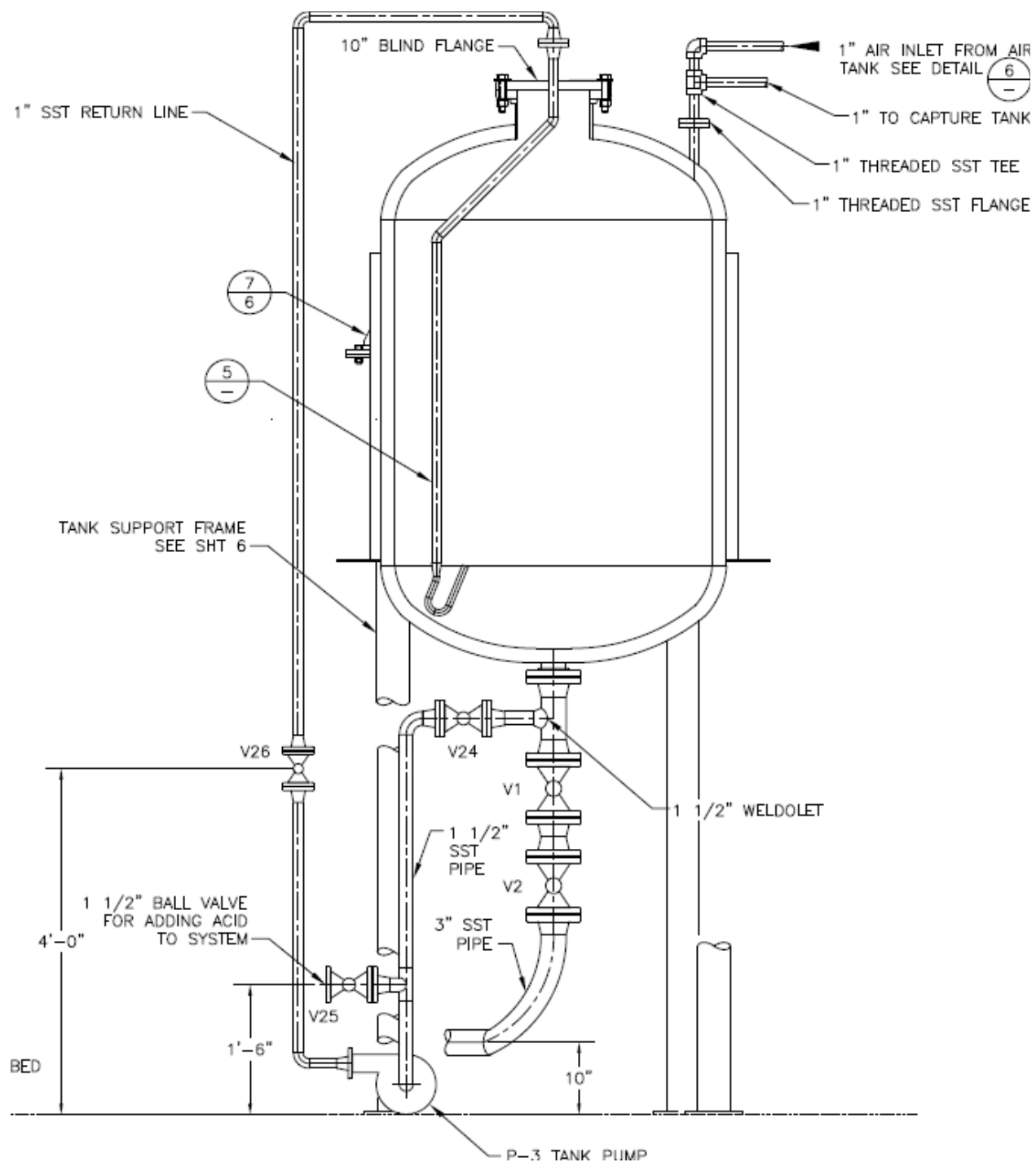

Figure 5.5. Schematic of Mixing Tank Internal Components

\subsection{Lasentec}

The Mettler Toledo Lasentec ${ }^{\mathrm{TM}}$ focused beam reflectance measurement (FBRM) system consists of a D600PST electronics package with a D600S probe (see Figure 5.6). The probe is mounted through a fitting at a $45^{\circ}$ angle into a vertical up-flow pipe leg. The tip of the probe contains a sapphire window with a rotating laser. As particles pass in front of the probe window, the laser beam is reflected back to the probe tip. Since the speed of the rotating laser is known, the size of the particle can be calculated by the duration of the reflected laser signal (see Figure 5.7). By counting the number of reflected laser signals, a histogram of particle size and counts can be obtained. The system has the capability of updating this histogram in 2-second intervals. 


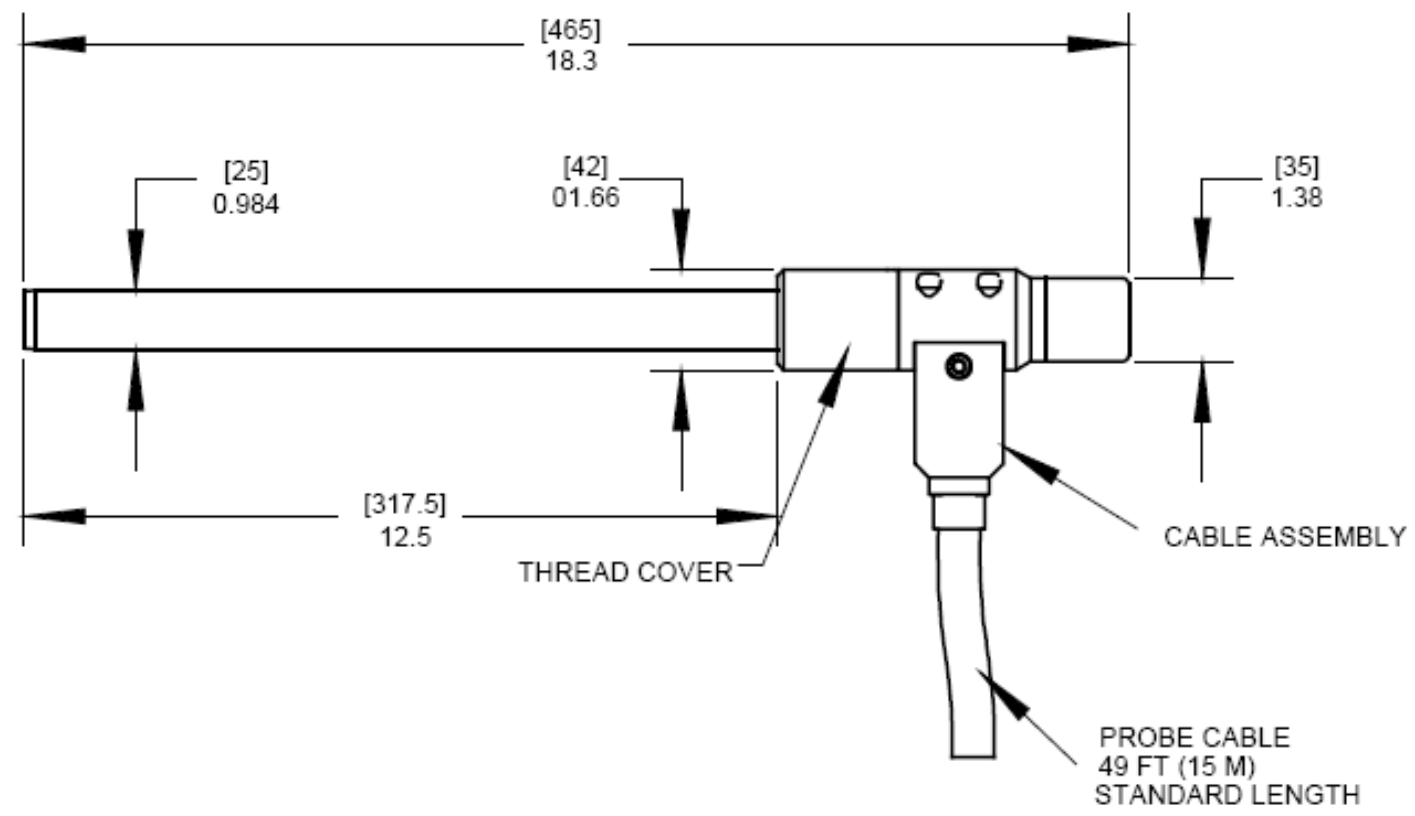

Figure 5.6. Schematic of Lasentec ${ }^{\mathrm{TM}}$ D600S Probe (source: Lasentec ${ }^{\mathrm{TM}}$ Operation Manual)

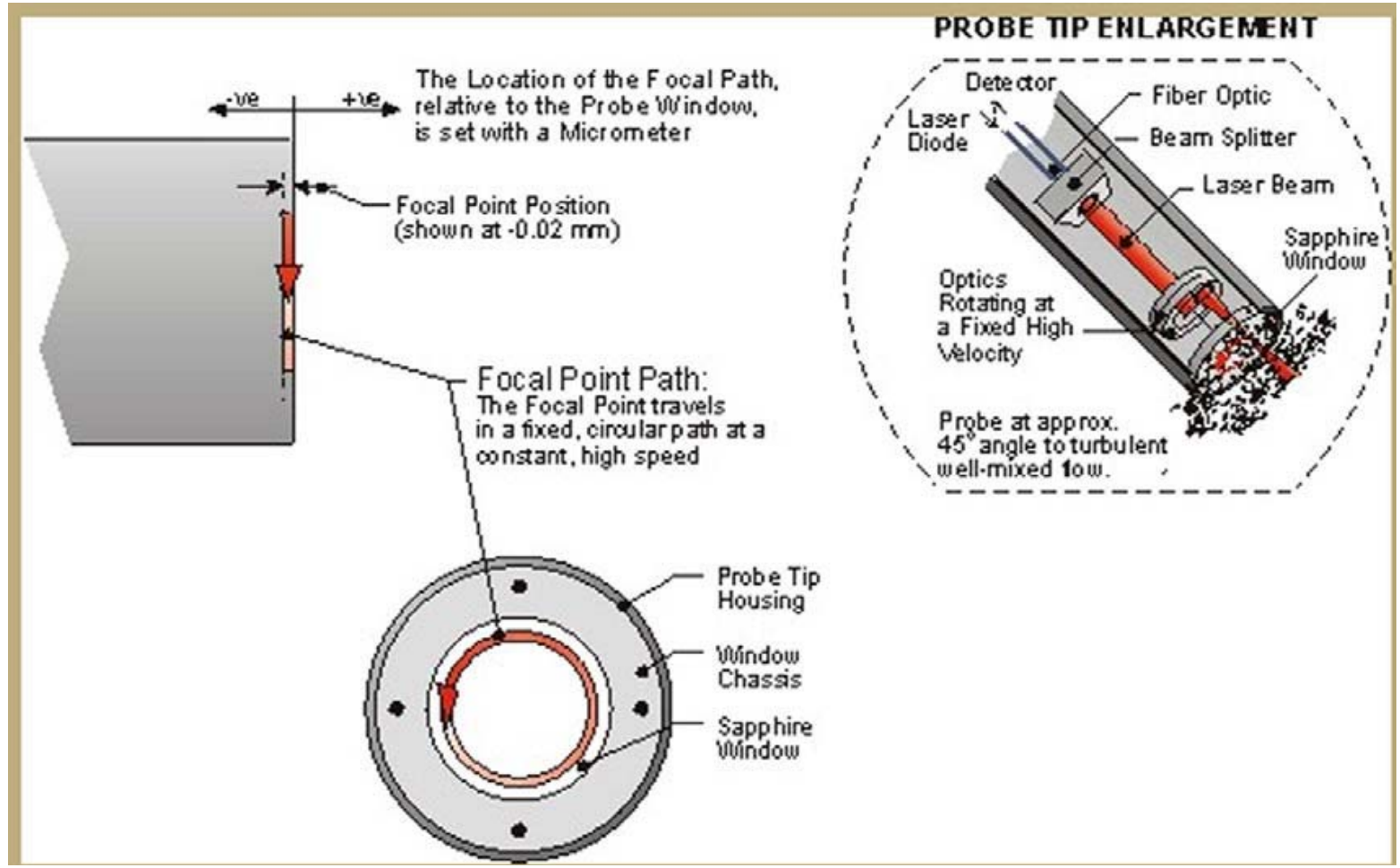

Figure 5.7. Schematic of Lasentec ${ }^{\mathrm{TM}}$ Measurement Concept (source: Lasentec ${ }^{\mathrm{TM}}$ Operation Manual)

Since the laser is randomly scanning across particles, the maximum length of the particle has a lower probability of being measured. For this reason, the system measures the chord-length distribution of the slurry particles rather than the PSD. Consequently, this system is very good for determining when 
process conditions have changed significantly. For the flow-loop experiments, the Lasentec ${ }^{\mathrm{TM}}$ system was used to assess whether significant particle attrition was occurring.

For the Lasentec values to be reportable, the system must be performance checked to NIST-traceable standards. Since no chord-length distribution NIST standards currently exist, the Lasentec data was used to determine when changes in the system occur. Consequently, the Lasentec data are categorized "for information only." Data for each test are shown in Appendix B.

\subsection{Electrical Resistance Tomography}

The electrical resistance tomography (ERT) system, model P2000, was manufactured by Industrial Tomography Systems (ITS). The system consists of three major components, a computer running the ITS software package, an electronic data-acquisition system, and the ERT sensor. The sensor used in the flow loop was configured in a pipe geometry that allows for the conductivity of the slurry to be mapped over the cross-sectional area of the pipe (see Figure 5.8). Since conductivity is a function of solids concentration, this map can be converted mathematically to vol\% solids. For these values to be reportable, the conductivity of the system must not vary over time, and the system must be performance checked to NIST-traceable standards. Due to difficulties in both of these areas, the ERT data are left as the original conductivity map form and was used for visualization purposes only. For this reason, the ERT data are categorized "for information only."
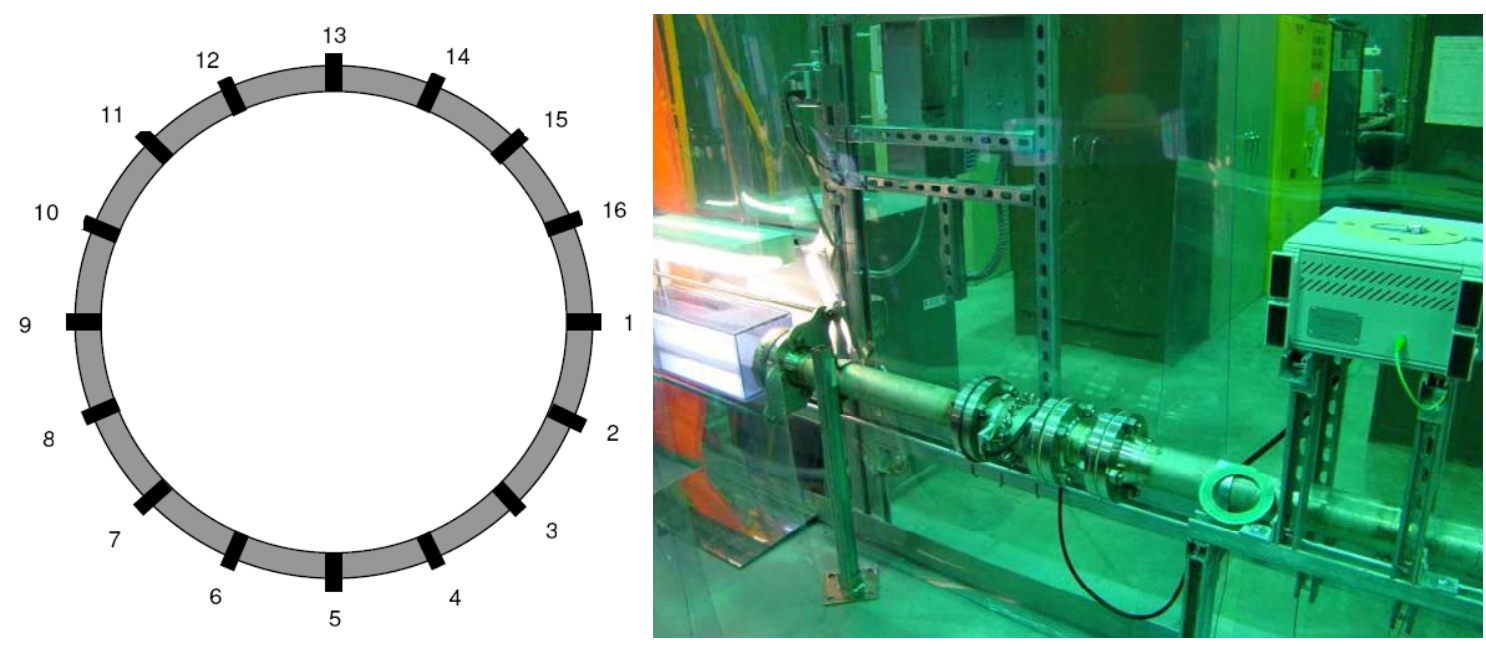

Figure 5.8. Right - Schematic of ERT Pipeline Sensor Electrode Layout. (source: P2000 Operation Manual)

Left - Installed PVC Transparent Pipe Section, ERT Pipeline Probe, and ERT Electronic Data-Acquisition System 


\subsection{Test Procedure}

Both critical velocity and flushing capability were assessed in a single test. This was accomplished by operating the system according to the sequence of steps outlined below:

1. Load Simulant for Testing

The simulant recipes were created with the information in Section 4.0. A simulant with the appropriate water-to-solids concentration was loaded into a rinsed and drained mixing vessel and flow loop. Tap water was used as the flush solution and was added to the flush vessel.

2. Homogenize Simulant

The mechanical agitator on the mixing vessel was started for solids homogenization. The mixingvessel temperature control system was set to the appropriate value, nominally $25^{\circ} \mathrm{C}$.

3. Confirm Homogenization

The mixing vessel flow loop was operated to determine slurry density in the vessel via Coriolis flow meters. Agitator rotational rates were adjusted such that the solid-volume fractions were within $20 \%$ of the theoretical calculated values for complete homogenization as well as to minimize the entrainment of air in the pipe loop.

4. Take Samples and Measure Rheological Properties

A grab sample was taken from the mixing vessel, and a flow curve (see Appendix A) was measured.

5. Start Pipe Flow

The flow loop pumps were started with a target flow velocity of $8 \mathrm{ft} / \mathrm{sec}$. Coriolis meters on the flow loop were used to verify this reading.

6. Flow/Instrumentation Measurements

The state of the flow was assessed with multiple techniques. A list of these techniques and a description of the significance of the measurement data are shown in the steps below.

a. Superficial Flow Velocity $(\mathrm{V})$ versus pipeline pressure drop $(\Delta \mathrm{P})$-A pressure drop over a known length of pipeline as a function of pipeline velocity is the primary measurement technique. These measurements were coupled with flow-rate data to ultimately provide a plot of flow rate versus pressure drop. An example of a typical slurry-transport plot is shown in Figure 6.1. 


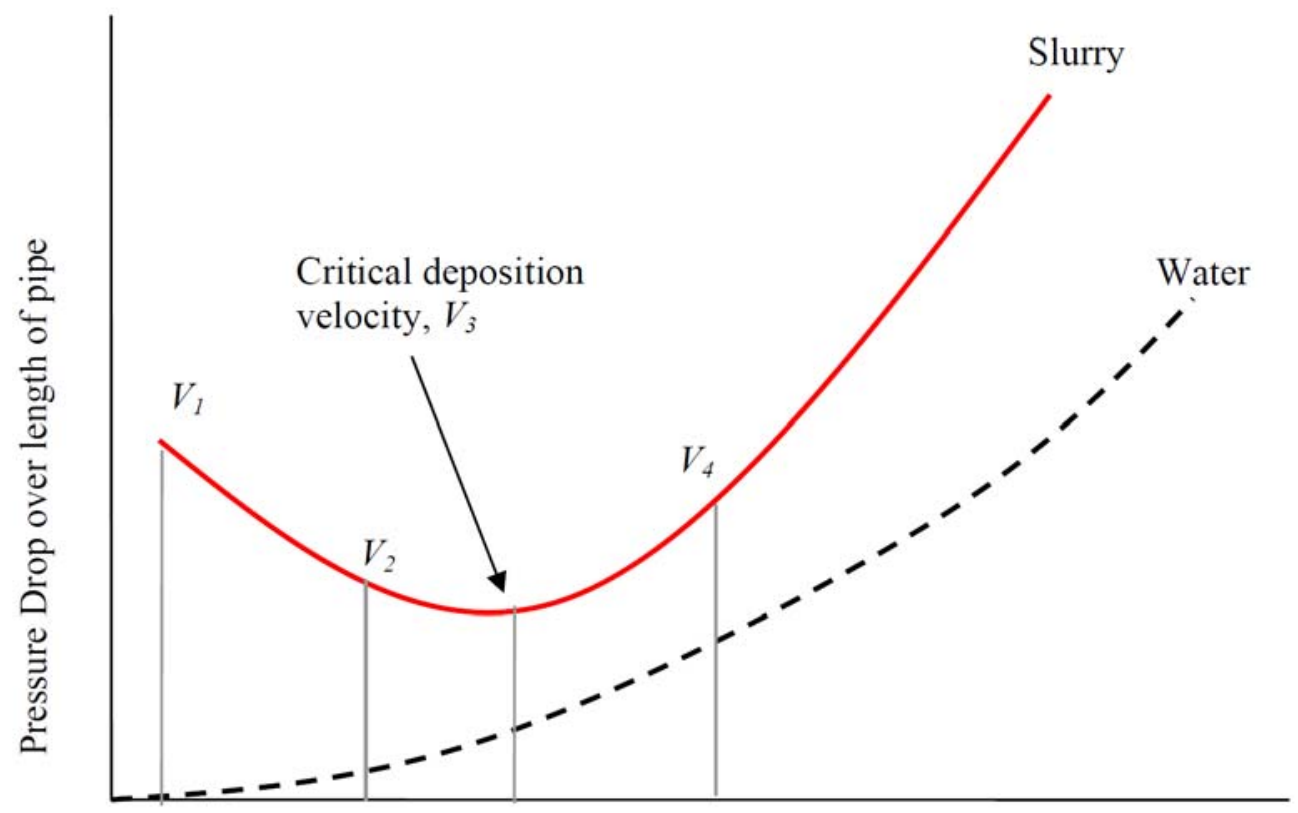

\section{Superficial Velocity}

Figure 6.1. Pressure Drop as a Function of Pipeline Superficial Velocity

A description of the velocity boundaries observed in such plots is discussed by Abulnaga (2002) as follows:

- $V_{1}$ : velocity at or above which the bed in the lower half of the pipe is stationary. In the upper half of the pipe, some solids may move by saltation or suspension.

- $V_{2}$ : velocity at or above which the mixture flows as an asymmetric mixture with the coarser particles forming a moving bed.

- $V_{3}$ : velocity at or above which all particles move as an asymmetric suspension and below which the solids start to settle and form a moving bed. This velocity corresponds to the critical velocity correlations of Oroskar and Turian (1980), Thomas (1979), and Shook et al. (2002) (see Equations (3.1), (3.2), and (A.23), respectively).

○ $V_{4}$ : velocity at or above which all solids move as a symmetric suspension.

b. Visual data - a video of the transparent test section was taken to assess the flow condition in the pipe at a particular flow rate.

c. Flow tomographs (spatial conductivity maps within the pipe cross-sectional area) were measured using the ERT system. As discussed previously, this system was used for visualization purposes only.

7. Determine Steady-State Condition

A flow rate was held constant for a minimum of 30 minutes before a steady-state condition was assumed. If the transient pressure signal indicated a trend after this 30-minute period, the flow was held constant until a steady state was declared by the cognizant scientist.

The temperature of the slurry was held constant during this testing via automatic temperature control on the jacketed mixing vessel. The temperature was maintained within $\pm 10^{\circ} \mathrm{C}$ of target temperature. 
8. Decrease Flow rate

The flow rate was decreased in $1 \mathrm{ft} / \mathrm{sec}$ or smaller increments, and steps 6 and 7 were repeated until the stationary bed/plug-velocity condition was reached. This point corresponds to velocity $V_{l}$ described in step 6 .

9. Stop Flow

The pump was then stopped with the line partially filled with sediment.

10. Switch to Flush Mode

Valving was reconfigured to operate in flush mode. This mode allowed for flow from the flush tank to go to the spent simulant vessel. The flush tank was filled with water and then driven through the system at the specified operating pressure.

\section{Measure Sediment Concentration Transported From Pipeline}

Flush density measurements-A Coriolis meter was used to measure the transient flow rate of the flush solution to the test loop. A second Coriolis meter was used to measure the density of the simulant into the spent simulant vessel. Since the density of the flush solution is known, an assessment of the effectiveness of the flush can be determined. 



\subsection{Newtonian Results and Discussion}

The waste simulants described in Section 4.0 were used in the test apparatus described in Section 5.0. Using the test procedure discussed in Section 6.0, critical-velocity measurements were obtained for each condition. These results have been segregated into Newtonian and non-Newtonian cases. In this section, results for Newtonian slurry transport are presented and discussed. Note that a test with water was conducted to baseline the system; results of this test are given in Appendix C. From this test the pipe relative roughness was determined to be approximately $1.2 \times 10^{-4}$. This value is in the midpoint range between commercial steel and drawn tubing. This roughness value was used for both Newtonian and non-Newtonian pressure-drop calculations.

\subsection{Newtonian Results}

The pressure drop over a 224.75-inch section of straight, horizontal, schedule 40, 3-inch pipe was measured at multiple pipeline velocities for the Newtonian slurries discussed in Table 4.4. These data as well as corresponding electrical resistivity tomograms are shown in Figure 7.1 to Figure 7.5. Note that transitions from one shaded area to another indicate a change in electrical conductivity in the pipe cross section. Conductivity is proportional to solids concentration or the presence of bubbles. Consequently, solid (dark) shading in the bottom of the pipe indicates the formation of a solid bed. Conversely, shading on the top of the pipe likely indicates the presence of bubbles in the pipe. Also note that the ERT images only show conductivity gradients in the pipe which are proportional to solids concentration. The ERT probes do not measure the solids velocity. Hence, a gradient will be observed on the ERT tomograms in regions of asymmetric or sliding bed flow. This region corresponds to the areas $V_{l}$ to $V_{4}$ in Figure 6.1.

Pressure-drop calculations based upon rheological measurements are also shown on these figures. The calculations were based upon the techniques described in Appendix A and are in excellent agreement with the experimental results. Based on the rheological properties determined for each simulant, the laminar-to-turbulent transition velocity was calculated with the equations outlined in Appendix A. For the "Low" rheology slurries, this transition velocity falls far below the measured critical deposition velocity.

Data for the LLL simulant system show that the critical velocity occurs at approximately $1.25 \mathrm{ft} / \mathrm{sec}$, where a minimum in the pressure drop versus flow rate occurs. ERT tomograms for this system clearly show the formation of a sediment bed in the bottom half of the pipe in this velocity range, and help to corroborate the interpretation of the pressure transducer data.

Data for the HLL simulant system show that the critical velocity occurs at approximately $2.5 \mathrm{ft} / \mathrm{sec}$. ERT tomograms for this system clearly show the formation of a sediment bed in the bottom half of the pipe in this velocity range. A gradient is observed developing in the top half of the tomograms. This could be due to the presence of bubbles either in the initial $8-\mathrm{ft} / \mathrm{sec}$ flow where the ERT system is "referenced" to a specified conductivity profile or from bubbles being drawn into the system at the lower flow rates. Regardless, the images of the bottom half of the pipe corroborate the interpretation of the pressure transducer data. 
The MML simulant system shows that the critical velocity occurs at approximately $3.0 \mathrm{ft} / \mathrm{sec}$. The LHL simulant system shows that the critical velocity occurs at approximately $4.0 \mathrm{ft} / \mathrm{sec}$. Again, these ERT tomograms show the progression of sediment-bed formation in the bottom half of the pipe in these velocity ranges.

The HHL simulant system shown in Figure 7.5 is unusual compared to the previous low rheology simulant results in that it exhibits a nearly constant pressure drop over much of the test-velocity range. Extreme deposition was observed at relatively high superficial velocities. As the velocity of the system drops further, the bulk density of the simulant system also drops due to deposition. The lower density reduces the amount of energy required to transport the slurry through the pipe. This mechanism offsets the increased pressure drop required because of the reduced cross-sectional area for flow. Because of the difficulties in identifying the critical deposition velocity via the $\Delta \mathrm{P}$ minimum in Figure 7.5 as a result of density decrease, an alternative analysis is employed to identify the deposition velocity. The friction factor, $f$, for a system will be the slope of the line formed by plotting $D \Delta P / 2 L \rho$ versus $V^{2}$. This can be seen by the definition of friction factor shown in Equation (A.43). If the data from Figure 7.5 are replotted in this format, the minimum value or critical velocity becomes apparent. From Figure 7.6, the critical velocity was determined to be approximately $8 \mathrm{ft} / \mathrm{sec}$. 


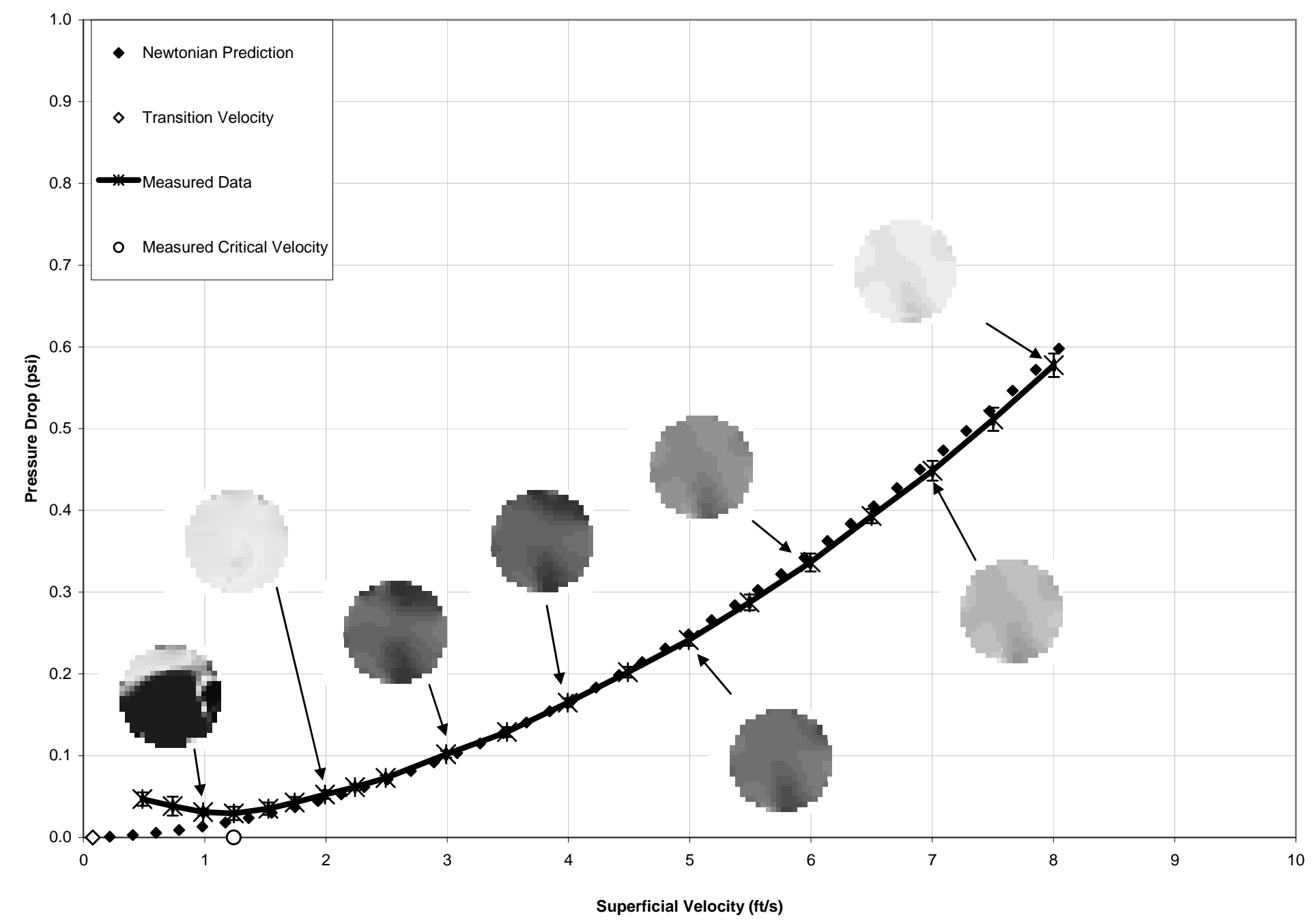

Figure 7.1. Pressure Drop and Electrical Resistivity Tomograms for LLL Simulant (10- $\mu \mathrm{m}$ glass beads in water) as a Function of Pipeline Velocity. Note that ERT tomograms are not calibrated to NIST standards and are considered "for information only." 


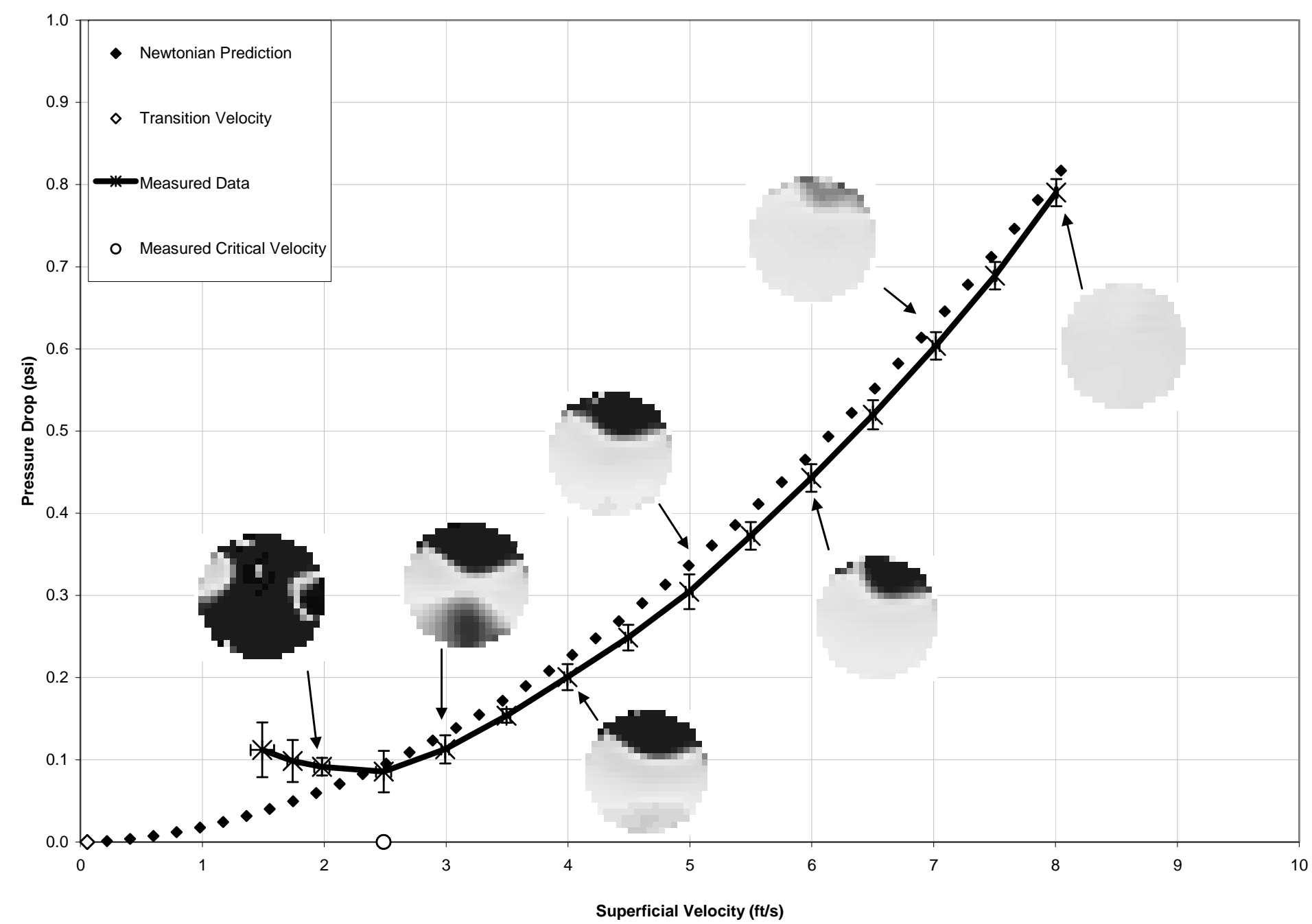

Figure 7.2. Pressure Drop and Electrical Resistivity Tomograms for HLL Simulant (10- $\mu \mathrm{m}$ stainless steel in water) as a Function of Pipeline Velocity. Note that ERT tomograms are not calibrated to NIST standards and are considered "for information only." 


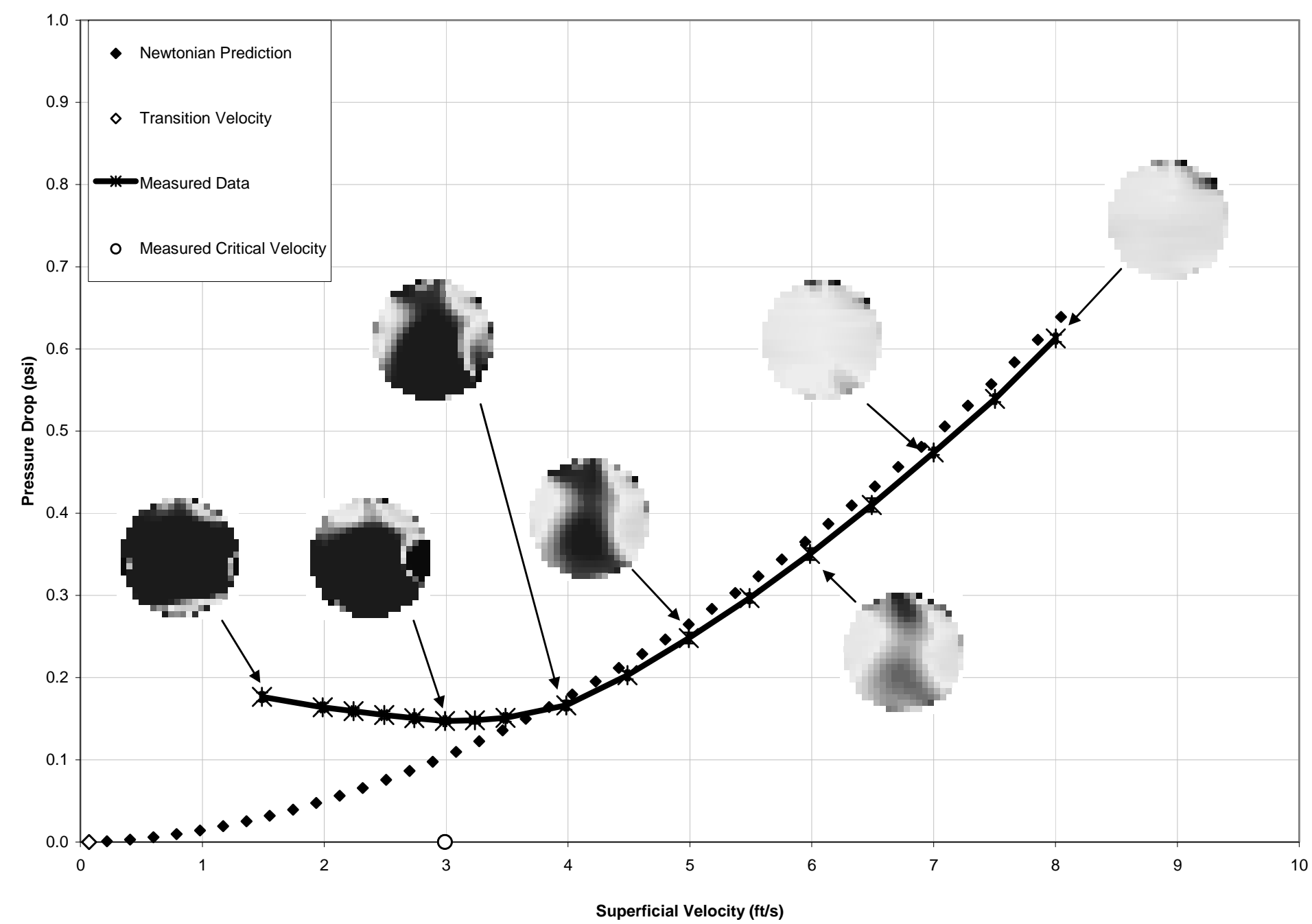

Figure 7.3. Pressure Drop and Electrical Resistivity Tomograms for MML Simulant (50- $\mu \mathrm{m}$ alumina in water) as a Function of Pipeline Velocity. Note that ERT tomograms are not calibrated to NIST standards and are considered "for information only." 


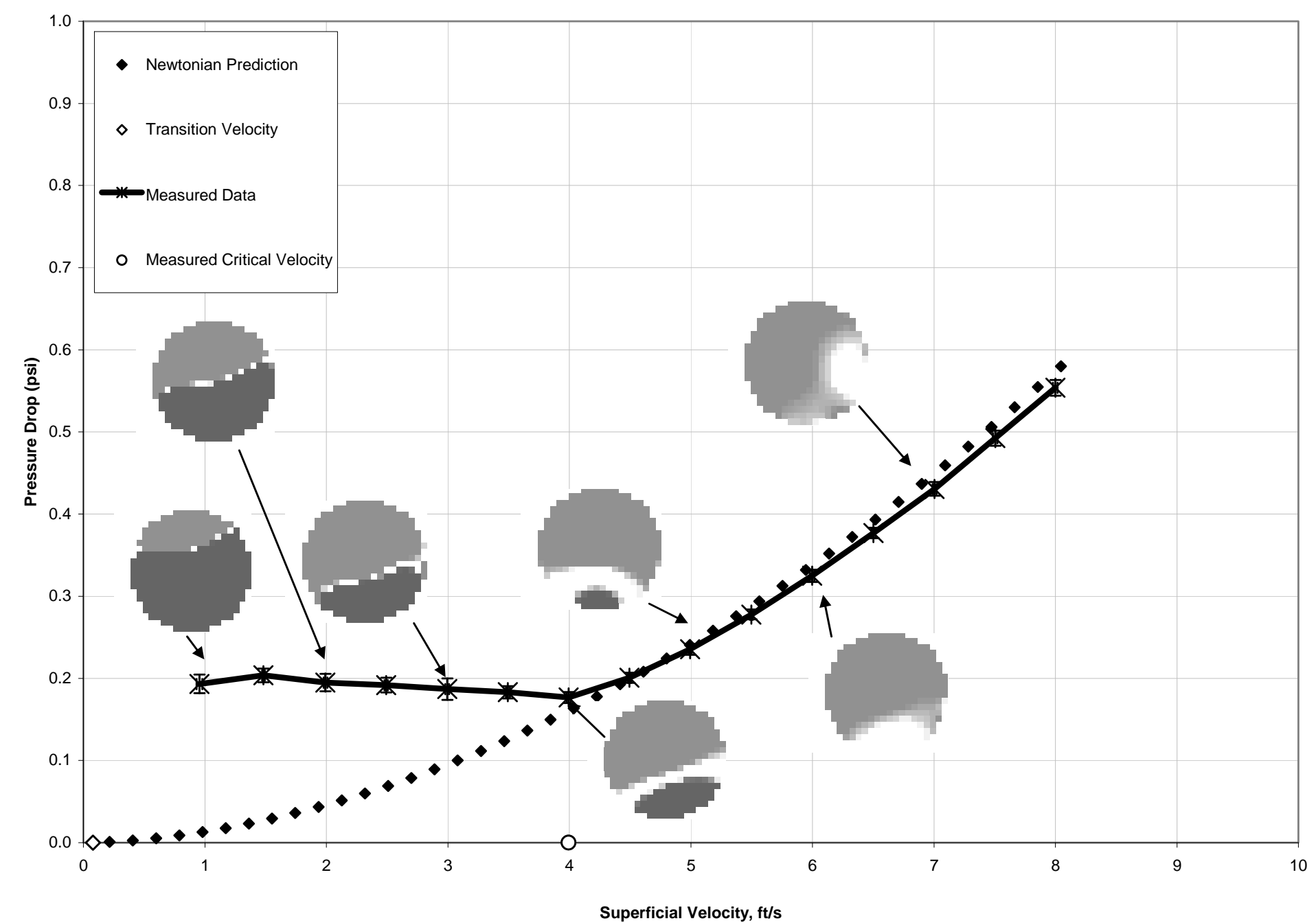

Figure 7.4. Pressure Drop and Electrical Resistivity Tomograms for LHL Simulant (100- $\mu \mathrm{m}$ glass beads in water) as a Function of Pipeline Velocity. Note that ERT tomograms are not calibrated to NIST standards and are considered "for information only." 


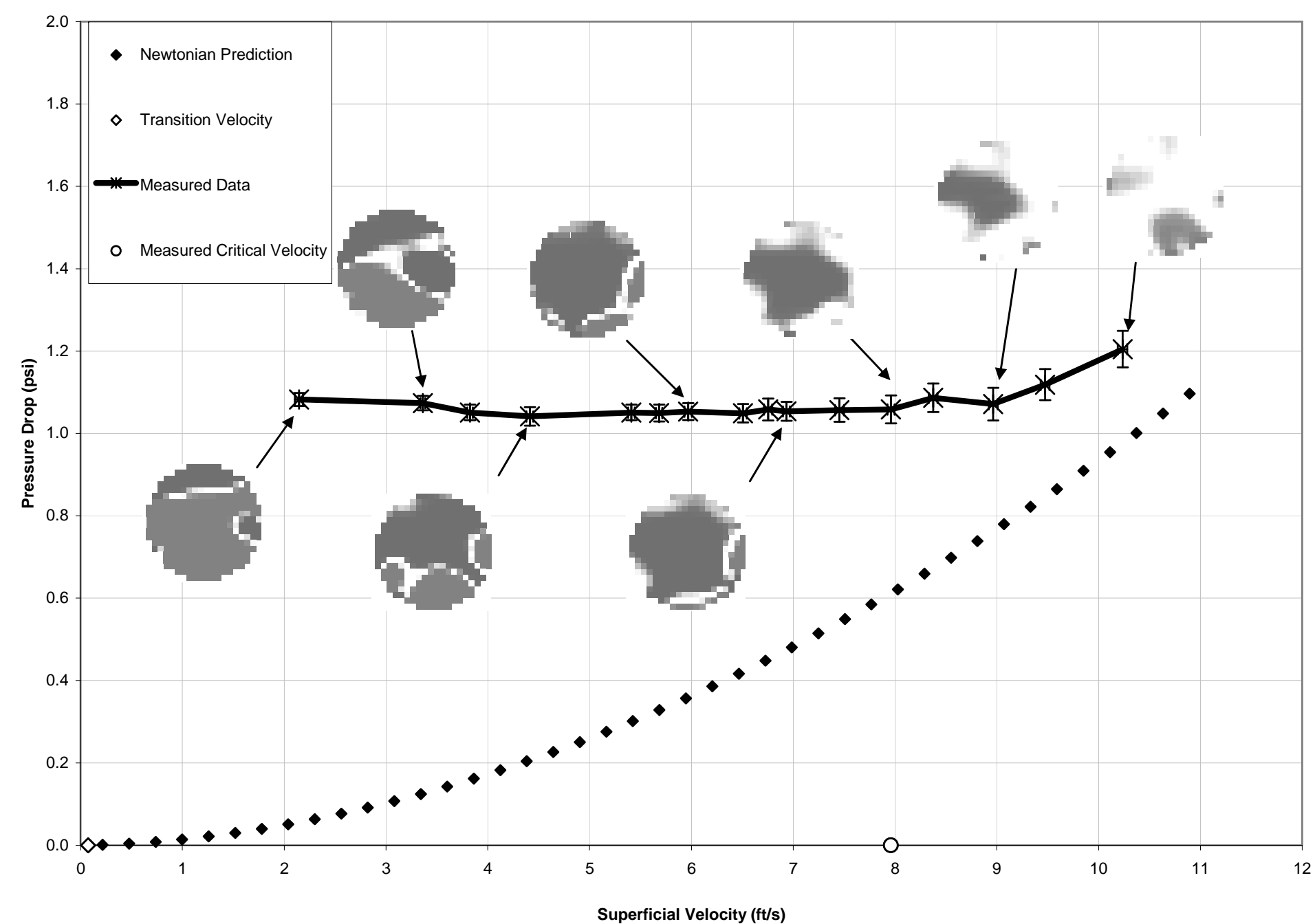

Figure 7.5. Pressure Drop and Electrical Resistivity Tomograms for HHL Simulant (100- $\mu \mathrm{m}$ stainless steel in water) as a Function of Pipeline Velocity. Note that ERT tomograms are not calibrated to NIST standards and are considered "for information only." 


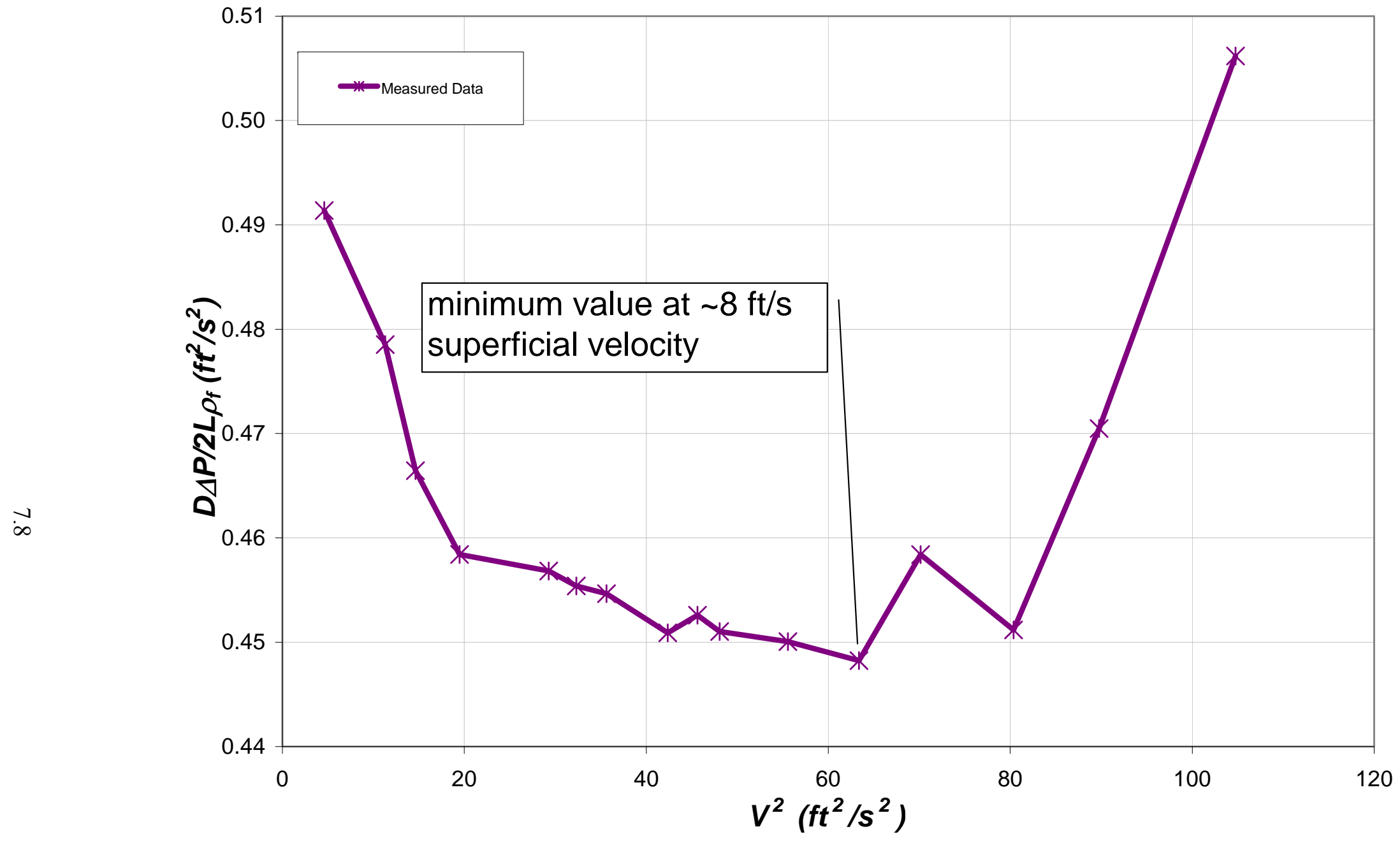

Figure 7.6. Plot that Accounts for Decreasing Fluid Density to Identify the Critical Velocity for the HHL Simulant (100- $\mu \mathrm{m}$ stainless steel in water) 


\subsection{Newtonian Discussion}

For each Newtonian system, the 24590-WTP-GPG-M-0058, Rev 0 design guide (Hall 2006) equations are applied to the simulant information in Table 4.4. These results are shown in Table 7.1 and graphically in Figure 7.7. The design-guide calculations with a $30 \%$ minimum margin are bounding for the experimental Newtonian data.

Table 7.1. 24590-WTP-GPG-M-0058, Rev 0 design guide (Hall 2006) Critical-Velocity Calculations for Newtonian Systems

\begin{tabular}{|c|c|c|c|c|c|c|}
\hline \multicolumn{2}{|l|}{ Test Configuration } & 1 & 2 & 3 & 4 & 5 \\
\hline \multicolumn{2}{|l|}{ Acronym } & LLL & HLL & MML & LHL & HHL \\
\hline \multicolumn{7}{|c|}{ (Step) Design-Guide Calculations } \\
\hline pipe diameter & $\mathrm{D}(\mathrm{m})$ & 0.078 & 0.078 & 0.078 & 0.078 & 0.078 \\
\hline (1) particle Diameter & $\mathrm{d}_{95}(\mu \mathrm{m})$ & 14.4 & 37.7 & 129.5 & 182.3 & 203.9 \\
\hline (2) average bulk density of dry solids & $\rho_{\mathrm{s}}\left(\mathrm{kg} / \mathrm{m}^{3}\right)$ & 2,500 & 7,950 & 3,770 & 2,500 & 7,950 \\
\hline (3) total solids volume fraction & $\mathrm{C}_{\mathrm{t}}$ & $9.8 \%$ & $9.3 \%$ & $8.7 \%$ & $7.4 \%$ & $3.0 \%$ \\
\hline (3) homogeneous solids volume fraction & $\eta_{\text {homo }}$ & $100.0 \%$ & $100.0 \%$ & $61.2 \%$ & $0.0 \%$ & $12.6 \%$ \\
\hline (3) heterogeneous solids volume fraction & $1-\eta_{\text {homo }}$ & $0.0 \%$ & $0.0 \%$ & $38.8 \%$ & $100.0 \%$ & $87.4 \%$ \\
\hline (3) solids volume fraction & $\mathrm{C}_{\mathrm{f}}$ & $9.8 \%$ & $9.3 \%$ & $5.3 \%$ & $0.0 \%$ & $0.4 \%$ \\
\hline (3) solids volume fraction & $\mathrm{C}_{\mathrm{r}}$ & $0.0 \%$ & $0.0 \%$ & $3.4 \%$ & $7.4 \%$ & $2.6 \%$ \\
\hline (4) carrier liquid density & $\rho_{\mathrm{f}}\left(\mathrm{kg} / \mathrm{m}^{3}\right)$ & 1,146 & 1,647 & 1,151 & 999 & 1,026 \\
\hline (5) carrier fluid viscosity & $\mu_{\mathrm{M}}(\mathrm{cP})$ & 10.2 & 9.3 & 4.5 & 1.5 & 1.6 \\
\hline (6) hindered Settling Factor & $\chi$ & 0.95 & 0.95 & 0.95 & 0.95 & 0.95 \\
\hline (7) critical velocity of fluid stream & $\mathrm{V}_{\mathrm{cr} 45}(\mathrm{ft} / \mathrm{sec})$ & 0.0 & 0.0 & 4.0 & 4.1 & 8.1 \\
\hline (8) limited critical velocity of fluid stream & $\mathrm{V}_{\mathrm{crT}}(\mathrm{ft} / \mathrm{sec})$ & 1.5 & 2.1 & 1.9 & 1.7 & 2.9 \\
\hline (9) apply design margin & $(\mathrm{ft} / \mathrm{sec})$ & 1.9 & 2.7 & 5.2 & 5.3 & 10.5 \\
\hline experimentally measured value & $(\mathrm{ft} / \mathrm{sec})$ & 1.2 & 2.5 & 3.0 & 4.0 & 8.0 \\
\hline
\end{tabular}

$\mathrm{n} / \mathrm{m}=$ not measured 
error bars indicate

$30 \%$ design margin

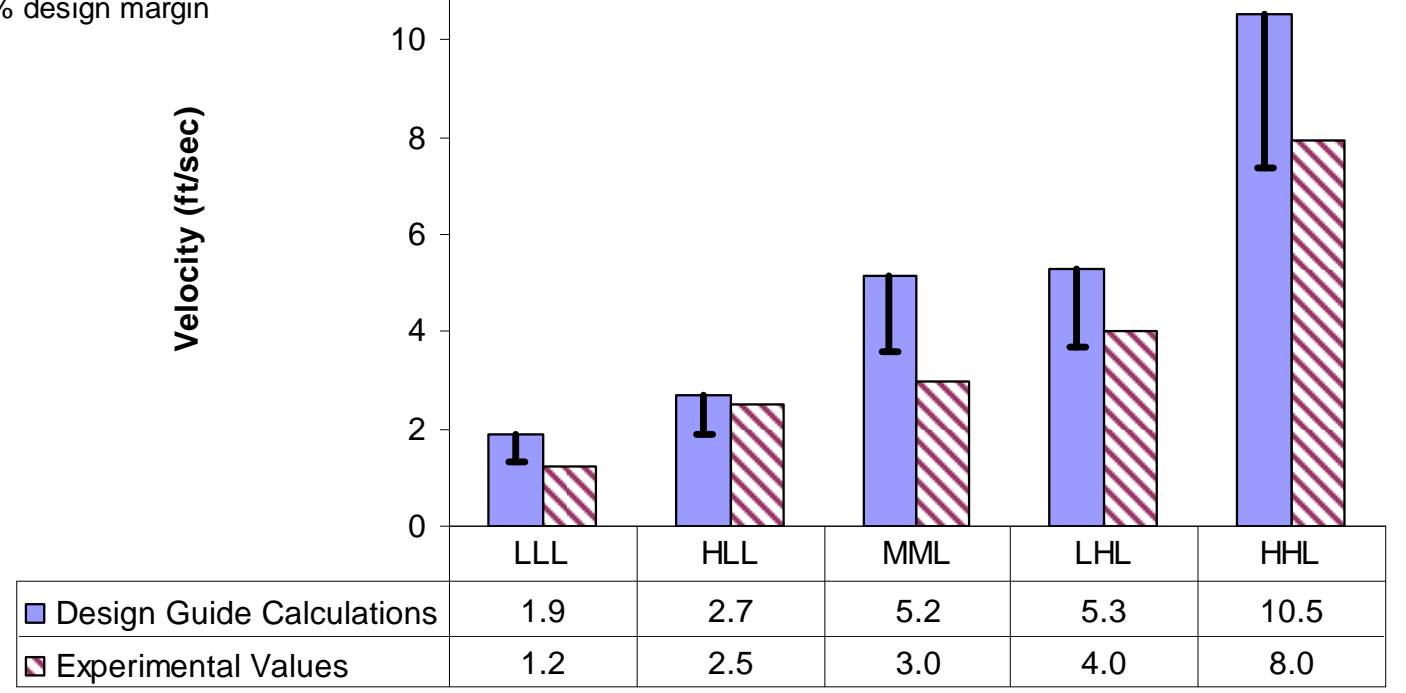

Figure 7.7. Experimentally Measured and Corresponding 24590-WTP-GPG-M-0058, Rev 0 Design Guide (Hall 2006) Calculated Values for Critical Velocity of Newtonian systems

One should note that a mathematical error exists in the equation to calculate the carrier-fluid density in step 4 of the 24590-WTP-GPG-M-0058, Rev 0 design guide (Hall 2006): the homogeneous portion solids volume fraction should be used in the denominator of Equation (8) rather than the heterogeneous portion solids-volume fraction. This substitution will appropriately close the mass balance on homogeneous particles. For the systems considered in this report, this correction only results in approximately a $5 \%$ increase in the calculated critical velocities. The source of this error is from an equation presented by Liddell and Burnett (2000).

From these data for Newtonian systems, the questions posed in Section 3.1 can be answered in the following manner:

1. Role of Density in Calculations - Since the waste simulants only consist of a single solid phase component, the calculated average density is the density of the coarse test particles. The questions raised in Section 3.0 on the topic of average density will be answered through the use of multi-component solid simulants in the non-Newtonian testing (see Section 8.0).

2. Homogeneous Fraction Concept-The slurries consisting of particles below $74 \mu \mathrm{m}$ that were considered to be "homogeneous" in the design guide are observed settling in the differential pressure drop and ERT data.

3. Use of RPP-9805 Viscosity Prediction-The predicted viscosities from the design guide approached $10 \mathrm{cP}$ while the measured values for these systems were approximately $1 \mathrm{cP}$. Since the predicted viscosity exceeds the measured value, the design guide critical-velocity values will be reduced and are potentially non-conservative. This illustrates one of the potential drawbacks to employing a viscosity "master" curve to evaluate carrier fluid viscosity. Specifically, when applied to systems beyond its region of chemical similarity, the RPP-9805 correlation (Jewett et al. 2002) may not provide a reasonable estimate of viscosity. This drawback is not limited only to the simulant systems 
tested herein but to any chemically dissimilar slurry, including HLW feeds, caustic and oxidative leached HLW solids, washed and leached HLW solids, HLW and LAW melter feed streams, ion exchange resin slurries, and solids-laden submerged-bed-scrubber solutions. This difficulty in accurately predicting viscosity of chemically dissimilar systems is to be expected, as the RPP-9805 (Jewett et al. 2002) viscosity correlation does not consider how suspending phase and particle chemistry impact bulk rheology (and viscosity). On the other hand, incorporation of even normal DLVO effects into a master rheology curve would be difficult and time consuming. Operationally, it may be more convenient to fit measured rheology data for HLW wastes processed in bench- or pilotscale test to the empirical formulas in Appendix A.3.

More importantly, the difficulties described above may not be limited to chemically dissimilar systems above. Even accurately predicting the viscosity of as-retrieved Hanford tank wastes with the RPP-9805 correlation may not be possible. As stated previously, Jewett et al. (2002) makes the following caveat about his correlation:

The viscosity correlations presented above have some weaknesses. One weakness is the relatively small number of tanks for which viscosities have been obtained for various defined solids loadings. Data for additional tanks would improve the quality of the final correlation. Furthermore, most viscosities used in this correlation were "apparent" and are equal to or greater than the true viscosity of the fluid.

Furthermore, Jewett et al. (2002) states “As information from future waste samples accumulates and as the simulant testing and theoretical studies continue, it may become possible to revise these values." The values to which Jewett et al. (2002) refers includes the best estimates of particle size, particle density, and slurry viscosity.

Poloski et al. (2007) compiled rheology data to evaluate the effectiveness of viscosity correlations in predicting waste tank rheological properties. Figure 7.8 shows the result obtained when the Poloski et al. (2007) rheology data set from 28 waste tanks is compared against the RPP-9805 correlation based on data from three waste tanks. The data common to both reports is shown by the solid diamond symbols. The vertical bands of data represent a single tank sample where the non-Newtonian apparent viscosity is calculated over a range of shear rates typical of pipeline transport systems (100 to $200 \mathrm{~s}^{-1}$ ). One should note that the common data, as presented in Poloski et al. (2007), are in a similar pattern to the RPP-9805 original data, but are shifted slightly but consistently to the left. This is due to differences between assumed particle densities to convert between mass fraction and volume fraction. The red circle symbols show the data added to the plot by Poloski et al. To minimize data clutter in the plot, apparent viscosities for "new" tanks were only evaluated at a single shear rate of $188 \mathrm{~s}^{-1}$. This shear rate represents the nominal shear rate for a fluid transported at $6 \mathrm{ft} / \mathrm{sec}$ in a 3 -inch schedule 40 pipe. The added data do not correlate with the RPP-9805 "master" curve and highlight the difficultly in developing a master viscosity curve based on solids concentration effects alone. Overall, caution must be applied when using the RPP-9805 to evaluate waste viscosity as it is not able to fully capture the range of viscosities observed in tanks for which viscosity data are available. 


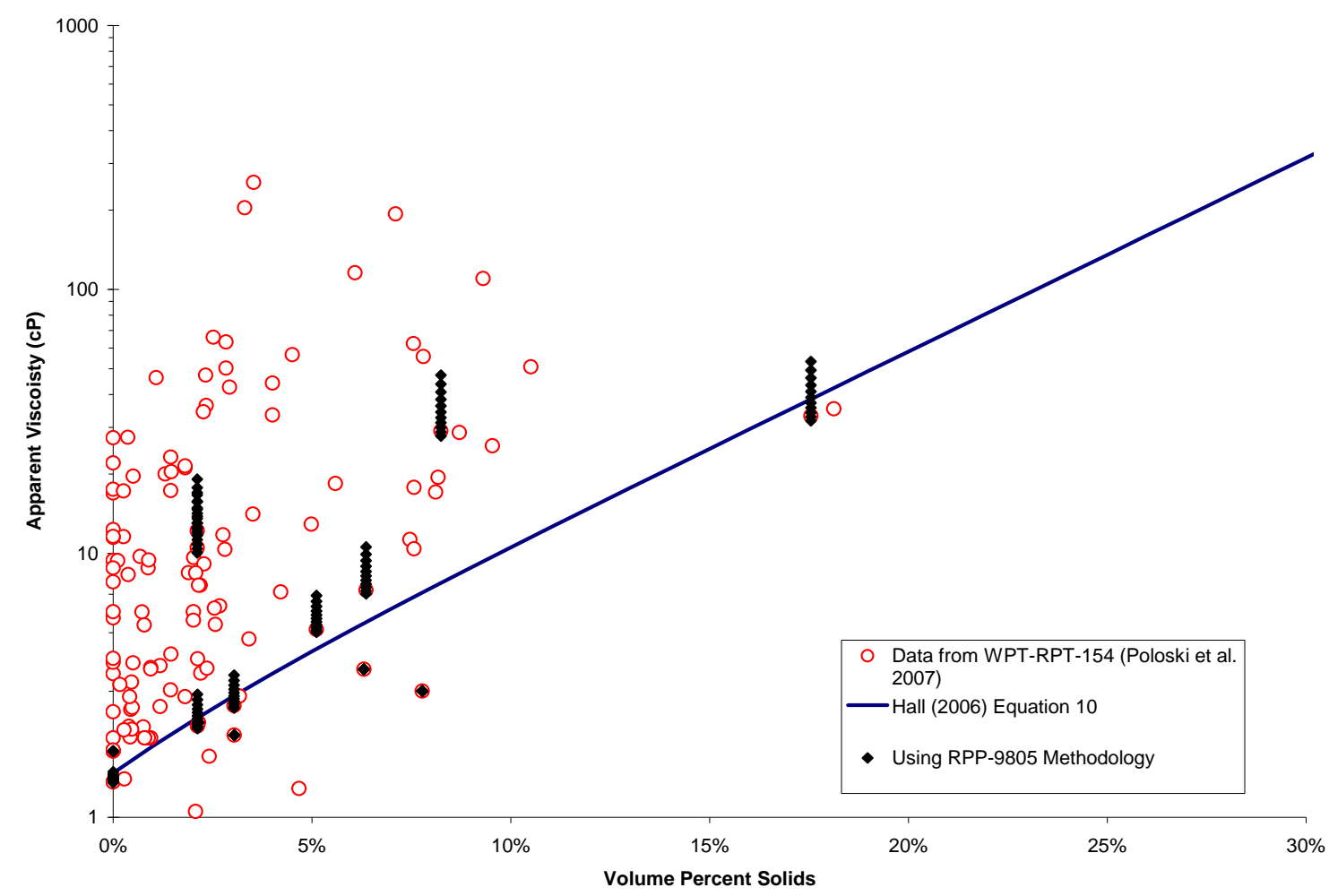

Figure 7.8. Experimentally Measured and Corresponding 24590-WTP-GPG-M-0058, Rev 0 Design Guide (Hall 2006) Calculated Values for Critical Velocity of Newtonian Systems 


\subsection{Non-Newtonian Results and Discussion}

The test simulants described in Section 4.0 were used in the test apparatus described in Section 5.0. Using the test procedure discussed in Section 6.0, critical-velocity measurements were obtained for each condition. These results have been segregated into Newtonian and non-Newtonian cases. In the previous chapter, the performance of the 24590-WTP-GPG-M-0058, Rev 0 design guide (Hall 2006) in predicting the critical deposition velocity of several Newtonian simulant slurries was evaluated. The results indicate adequate performance; however, use of the master viscosity curve introduces inaccuracies into the calculation that render a design flow velocity that is less accurate than expected. In this section, results for non-Newtonian slurry transport are presented and discussed.

\subsection{Non-Newtonian Results}

The pressure drop over a 224.75-inch section of straight, horizontal, schedule 40, 3-inch pipe was measured at multiple pipeline velocities for the non-Newtonian slurries with target yield-stress values of 3 Pa discussed in Table 4.5 (i.e., the "medium" rheology slurry). These data as well as corresponding electrical resistivity tomograms are shown in Figure 8.1 to Figure 8.5. Note that transitions from one shaded area to another indicate a change in electrical conductivity in the pipe cross section. Conductivity is proportional to solids concentration or the presence of bubbles. Consequently, solid (dark) shading in the bottom of the pipe indicates the formation of a solid bed. Conversely, shading on the top of the pipe likely indicates the presence of bubbles in the pipe.

Pressure drop calculations based upon rheological measurements are also shown on these figures. The calculations were based upon the techniques described in Appendix A and are in excellent agreement with the experimental results. Based on the rheological properties determined for each simulant, the laminar-to-turbulent transition velocity was calculated via the equations outlined in Appendix A. For several of the "medium" rheology slurries, this transition velocity is relatively close to the measured critical deposition velocity.

Data for the LLM simulant system (see Figure 8.1) show that the critical velocity occurs at approximately $1.5 \mathrm{ft} / \mathrm{sec}$. ERT tomograms for this system clearly show the formation of a sediment bed in the bottom half of the pipe in this velocity range. However, the pressure rise is so slight that a slidingbed regime might have occurred. For this reason, the data point is considered suspect, and critical velocity was not distinctly observed for this system.

Data for the HLM simulant system (see Figure 8.2) show that the critical velocity occurs at approximately $3.2 \mathrm{ft} / \mathrm{sec}$. ERT tomograms for this system clearly show the formation of a sediment bed in the bottom half of the pipe in this velocity range.

The MMM simulant system (see Figure 8.3 ) shows that the critical velocity occurs at approximately $2.7 \mathrm{ft} / \mathrm{sec}$. Again, these ERT tomograms show the progression of sediment-bed formation in the bottom half of the pipe in these velocity ranges. Magnesium sulfate salt $\left(\mathrm{MgSO}_{4}\right)$ is added to control rheology to the target yield-stress values. This salt addition increases conductivity from the initial ERT reference or baseline condition at the start of the run. This explains why the 8-ft/sec tomogram is showing increased conductivity (i.e. images have a darker background) that propagates throughout the run. Three runs on 
this system were performed with 2.7 and $3.0 \mathrm{ft} / \mathrm{sec}$ being the replicate and triplicate critical-velocity values. This demonstrates that the deposition velocity experiments discussed in this report are highly repeatable.

The LHM simulant system (see Figure 8.4 ) shows that the critical velocity occurs at approximately $3.5 \mathrm{ft} / \mathrm{sec}$. The HHM simulant system (see Figure 8.5 ) shows that the critical velocity occurs at approximately $4.5 \mathrm{ft} / \mathrm{sec}$. This is supported by the ERT tomograms that show the progression of sediment-bed formation in the bottom half of the pipe in these velocity ranges. 


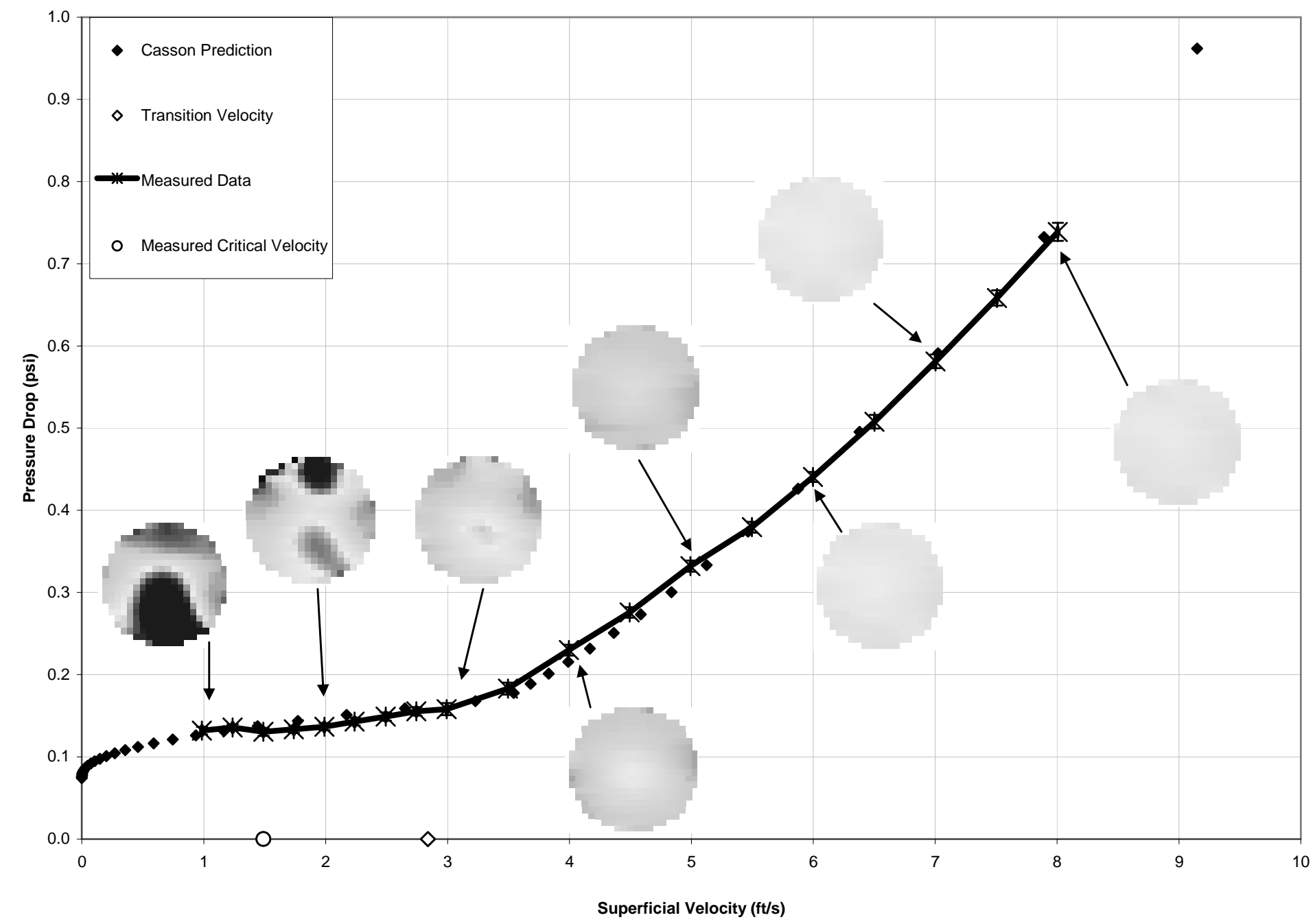

Figure 8.1. Pressure Drop and Electrical Resistivity Tomograms for LLM Simulant (10- $\mu \mathrm{m}$ glass beads in thin clay) as a Function of Pipeline Velocity. Note that ERT tomograms are not calibrated to NIST standards and are considered "for information only." 


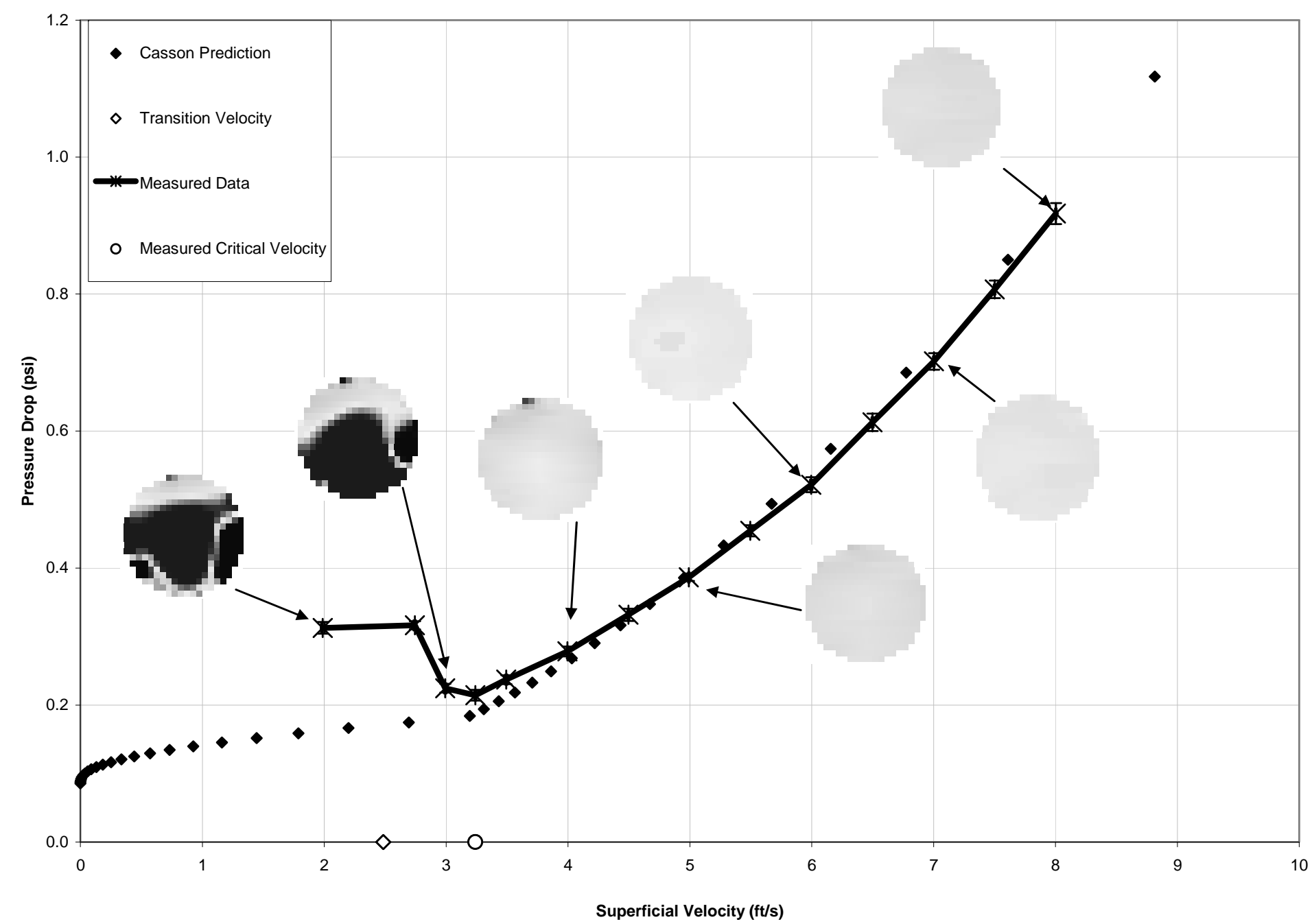

Figure 8.2. Pressure Drop and Electrical Resistivity Tomograms for HLM Simulant (10- $\mu$ m stainless steel in thin clay) as a Function of Pipeline Velocity. Note that ERT tomograms are not calibrated to NIST standards and are considered "for information only." 


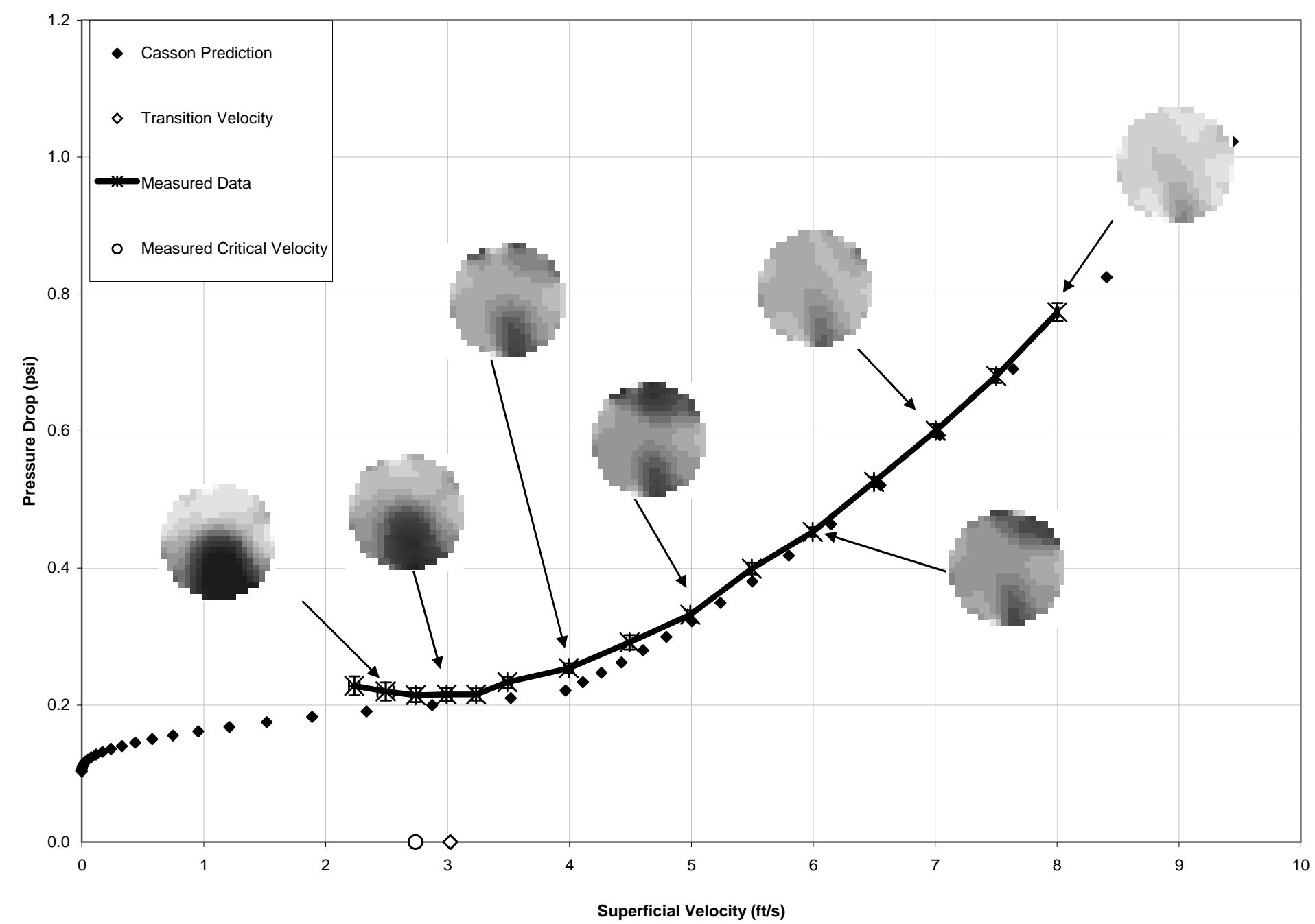

Figure 8.3. Pressure Drop and Electrical Resistivity Tomograms for MMM Simulant, Run \#1 (50- $\mu \mathrm{m}$ alumina in thin clay) as a Function of Pipeline Velocity. Note that ERT tomograms are not calibrated to NIST standards and are considered "for information only." 


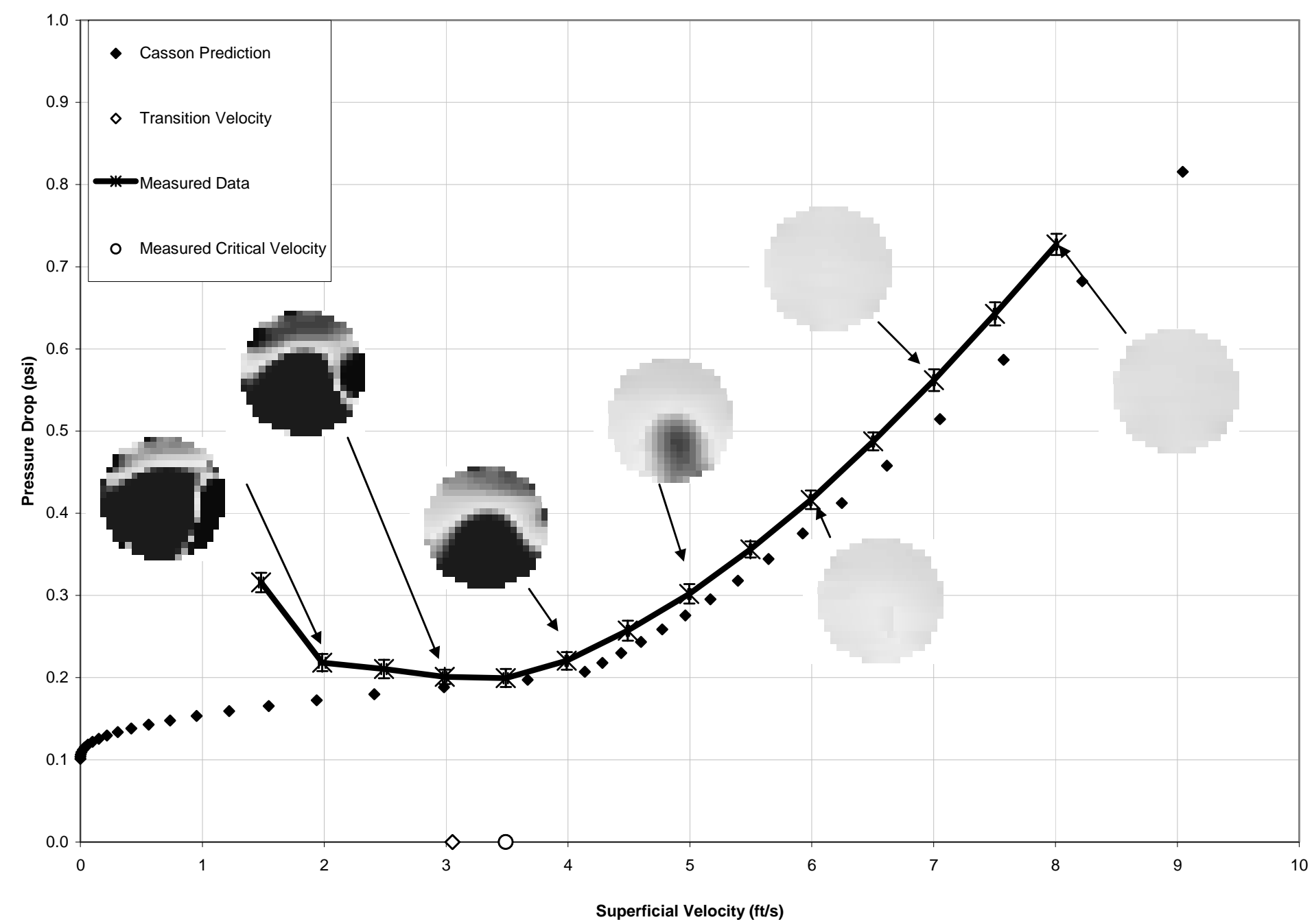

Figure 8.4. Pressure Drop and Electrical Resistivity Tomograms for LHM Simulant (100- $\mu \mathrm{m}$ glass beads in thin clay) as a Function of Pipeline Velocity. Note that ERT tomograms are not calibrated to NIST standards and are considered "for information only." 


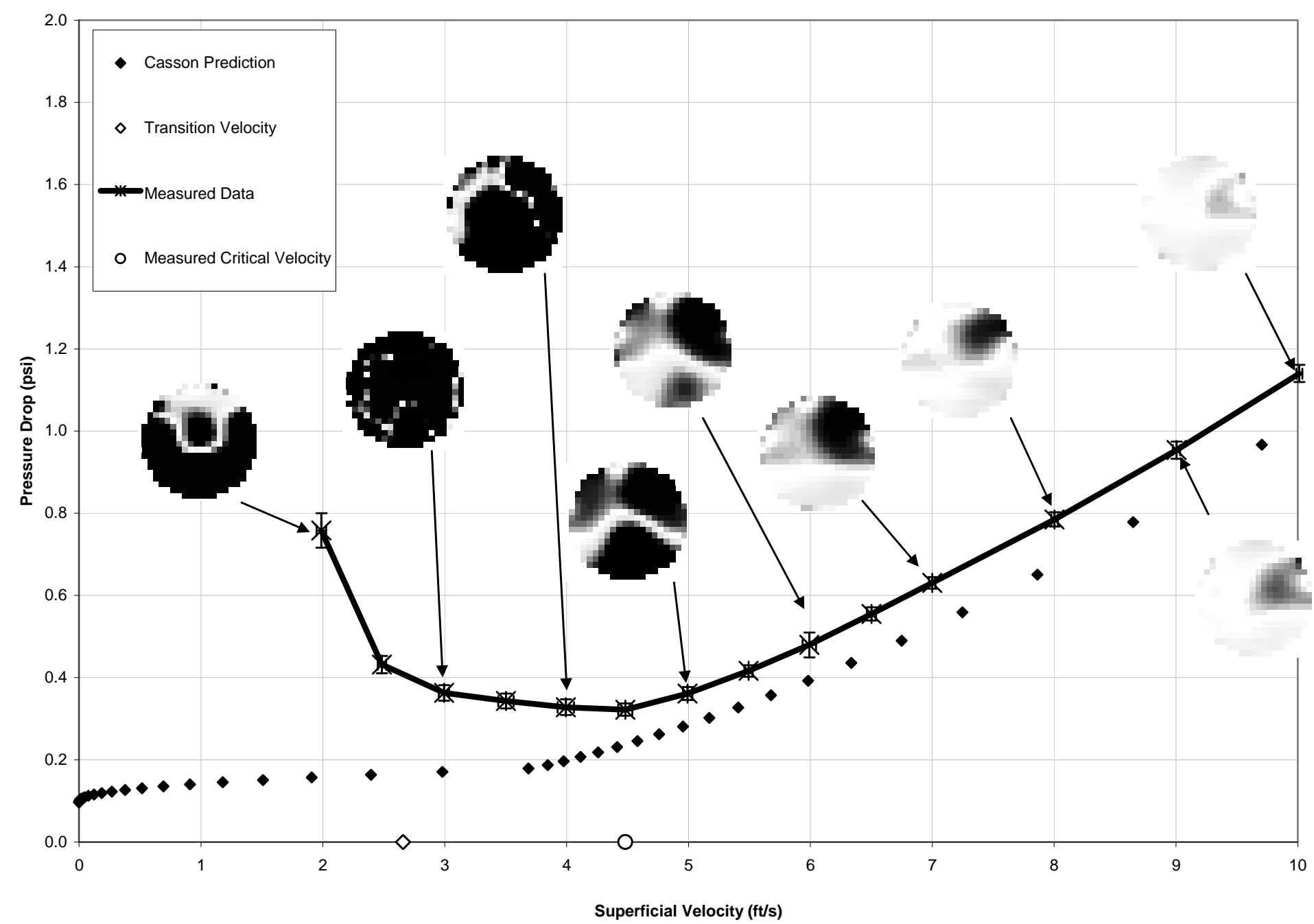

Figure 8.5. Pressure Drop and Electrical Resistivity Tomograms for HHM Simulant (100- $\mu \mathrm{m}$ stainless steel in thin clay) as a Function of Pipeline Velocity. Note that ERT tomograms are not calibrated to NIST standards and are considered "for information only." 
The pressure drop over a 224.75 -inch section of straight, horizontal, schedule 40,3 -inch pipe was measured at multiple pipeline velocities for the non-Newtonian slurries with target yield-stress values of $6 \mathrm{~Pa}$ discussed in Table 4.6 (i.e., the "High" rheology slurry). These data as well as corresponding electrical resistivity tomograms are shown in Figure 8.6 to Figure 8.10. Note that transitions from one shaded area to another indicate a change in electrical conductivity in the pipe cross section. Conductivity is proportional to solids concentration or the presence of bubbles. Consequently, solid (dark) shading in the bottom of the pipe indicates the formation of a solid bed. Conversely, shading on the top of the pipe likely indicates the presence of bubbles in the pipe.

Pressure-drop calculations based upon rheological measurements are also shown on these figures. The calculations were based upon the techniques described in Appendix A and are in excellent agreement with the experimental results. Based on the rheological properties determined for the each simulant, the laminar-to-turbulent transition velocity was calculated via the equations outlined in Appendix A. For several of the "High" rheology slurries, this transition velocity is relatively close to the measured critical deposition velocity.

Pressure-drop data for the LLH simulant system (see Figure 8.6) do not indicate that a critical velocity is present for this system. ERT tomograms for this system clearly show the formation of a sediment bed in the bottom half of the pipe in the velocity $2-$ to $1-\mathrm{ft} / \mathrm{sec}$ range. However, no corresponding rise in pressure is observed. For this reason, a critical-velocity datum was not distinctly observed for this system.

Data for the HLH and MMH simulant system (see Figure 8.7 and Figure 8.8, respectively) show that the critical velocity occurs at approximately $3.2 \mathrm{ft} / \mathrm{sec}$. The LHH simulant system (see Figure 8.9 ) shows that the critical velocity occurs at approximately $4.0 \mathrm{ft} / \mathrm{sec}$. Lastly, the HHH simulant system (see Figure 8.10) shows that the critical velocity occurs at approximately $5.0 \mathrm{ft} / \mathrm{sec}$. These data are supported by the ERT tomograms that show the progression of sediment-bed formation in the bottom half of the pipe in these velocity ranges. 


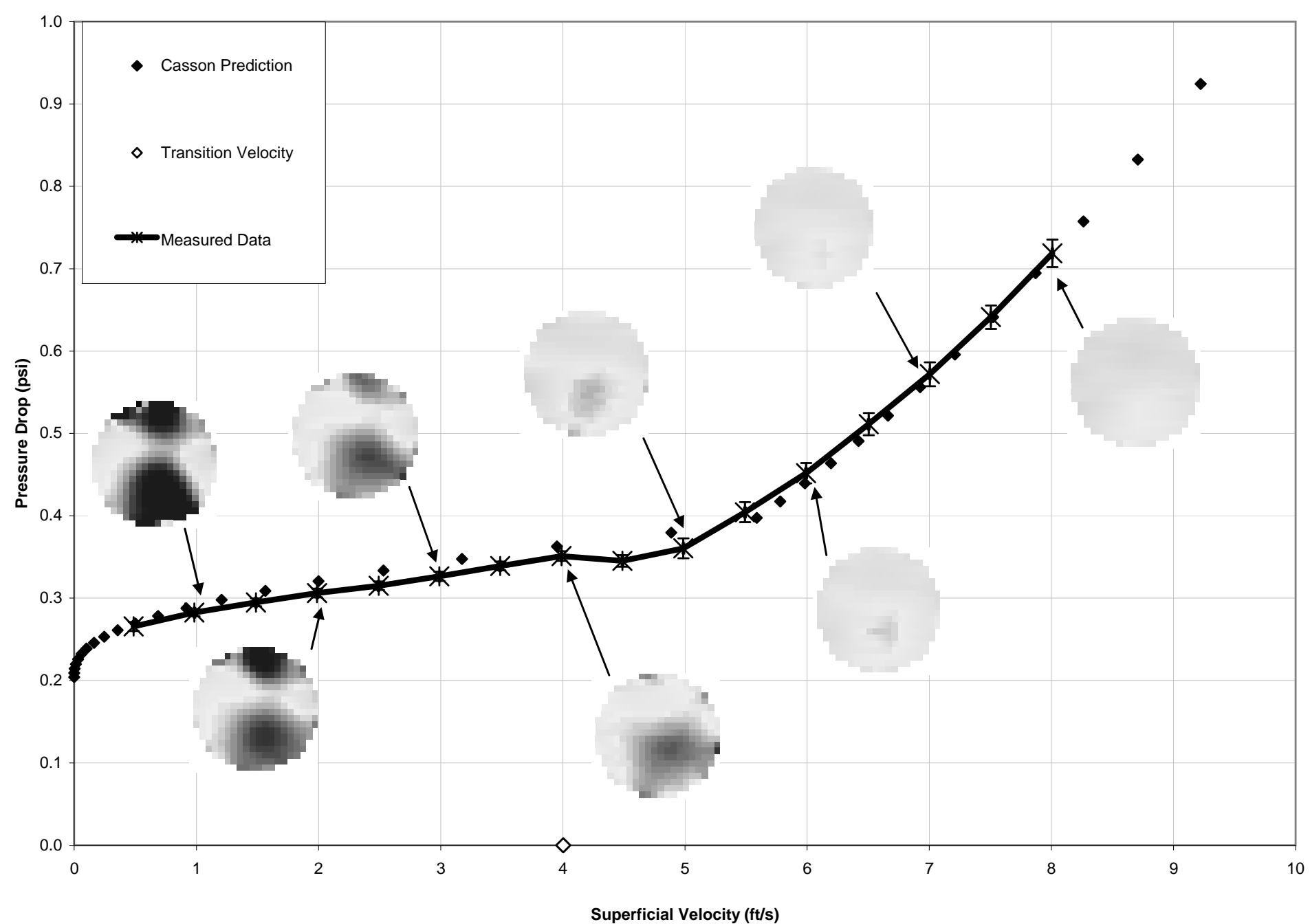

Figure 8.6. Pressure Drop and Electrical Resistivity Tomograms for LLH Simulant (10- $\mu$ m glass beads in thick clay) as a Function of Pipeline Velocity. Note that ERT tomograms are not calibrated to NIST standards and are considered "for information only." 


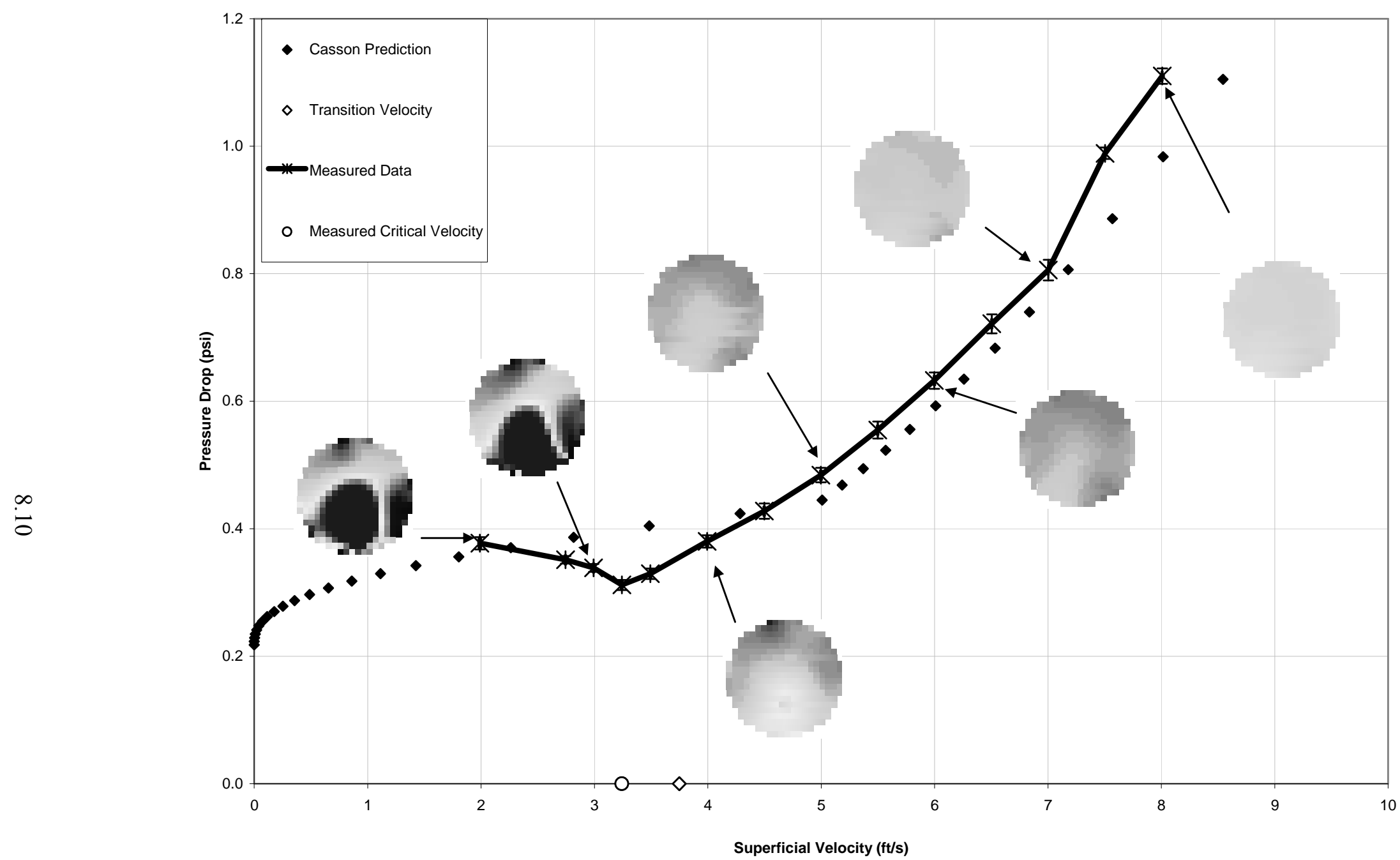

Figure 8.7. Pressure Drop and Electrical Resistivity Tomograms for HLH Simulant (10- $\mu$ m stainless steel in thick clay) as a Function of Pipeline Velocity. Note that ERT tomograms are not calibrated to NIST standards and are considered "for information only." 


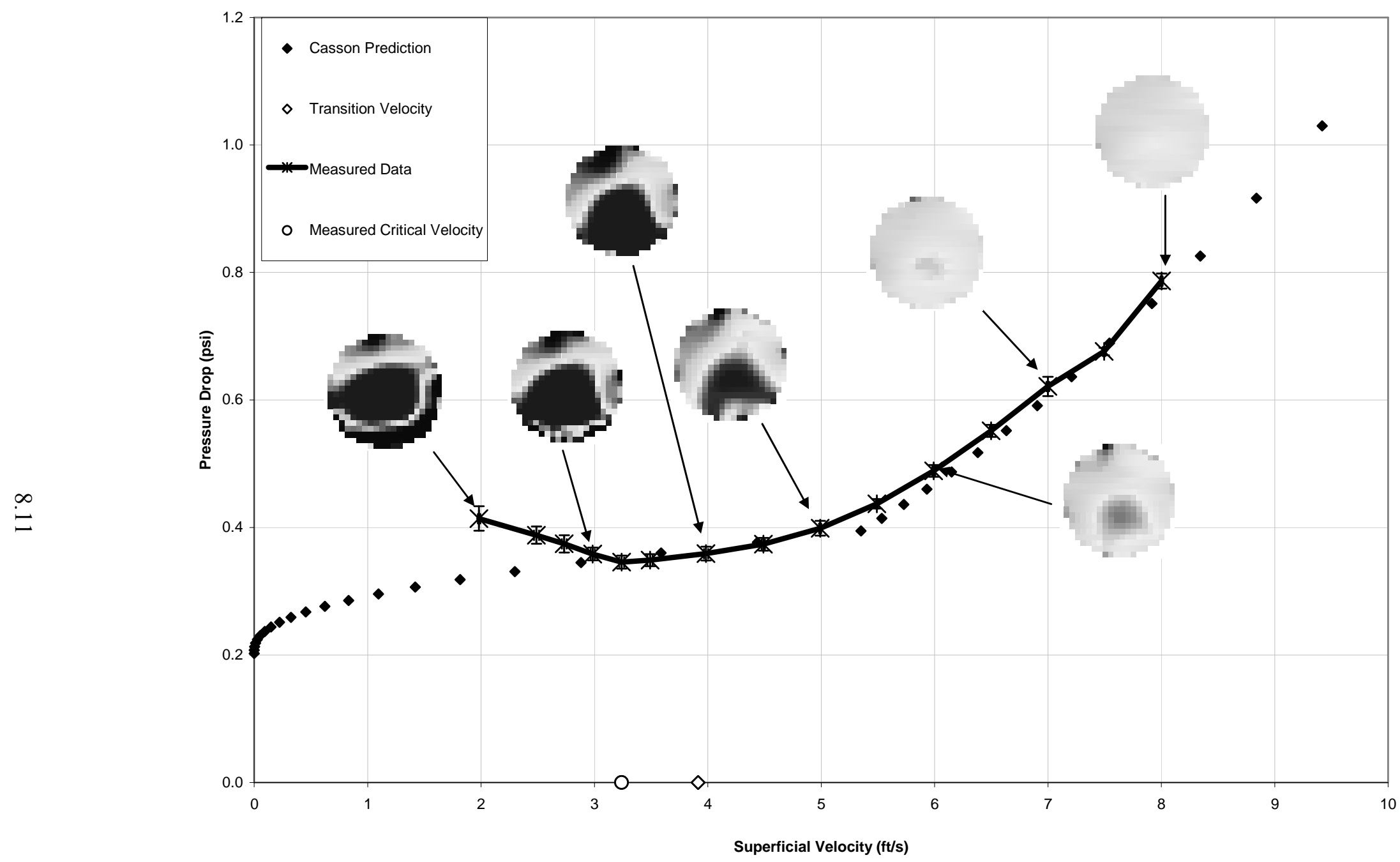

Figure 8.8. Pressure Drop and Electrical Resistivity Tomograms for MMH Simulant (50- $\mu$ m alumina in thick clay) as a Function of Pipeline Velocity. Note that ERT tomograms are not calibrated to NIST standards and are considered "for information only." 


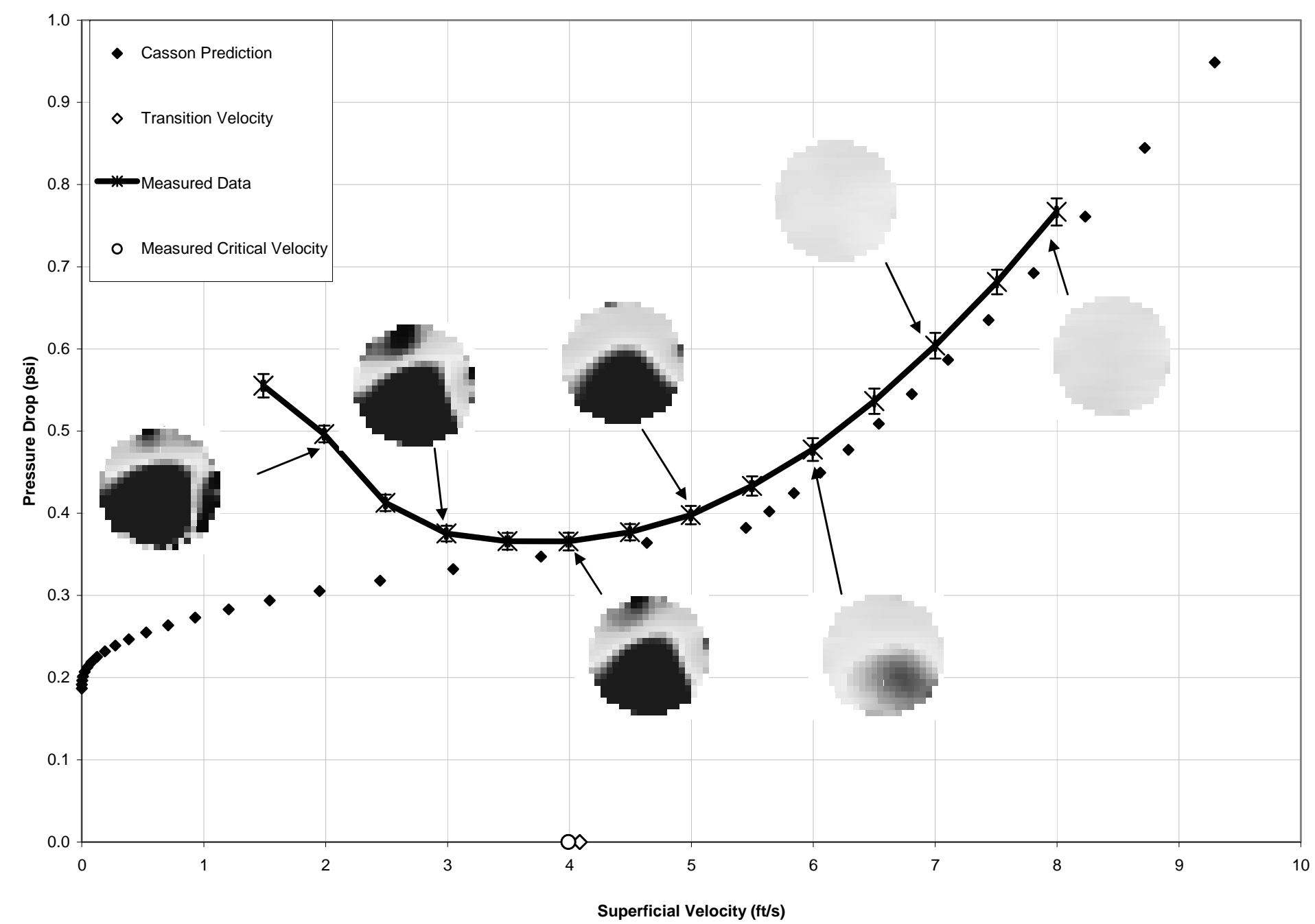

Figure 8.9. Pressure Drop and Electrical Resistivity Tomograms for LHH Simulant (100- $\mu \mathrm{m}$ glass beads in thick clay) as a Function of Pipeline Velocity. Note that ERT tomograms are not calibrated to NIST standards and are considered "for information only." 


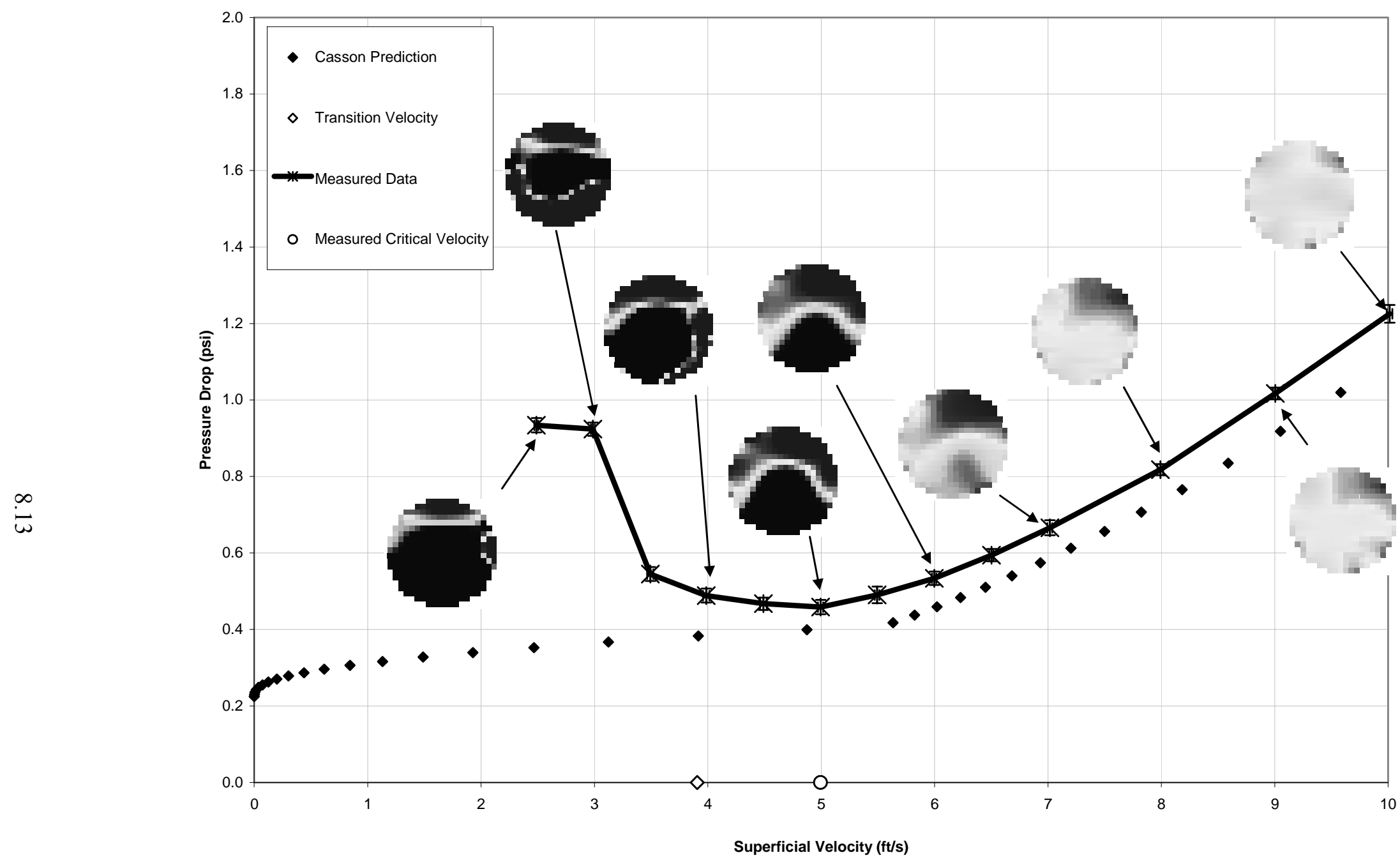

Figure 8.10. Pressure Drop and Electrical Resistivity Tomograms for HHH Simulant (100- $\mu \mathrm{m}$ stainless steel in thick clay) as a Function of Pipeline Velocity. Note that ERT tomograms are not calibrated to NIST standards and are considered "for information only." 


\subsection{Non-Newtonian Discussion}

24590-WTP-GPG-M-0058, Rev 0 design guide (Hall 2006) restricts the critical velocity calculations to Newtonian slurries. However, several major WTP process streams are known to possess highly nonNewtonian rheological properties. These include the HLW tank waste feed, HLW pretreated sludge, and HLW and LAW melter feeds. Because the design guide calculations are restricted to Newtonian systems, no WTP design guide exists for determining the critical velocity of non-Newtonian fluids within the WTP. Although the design guide may be adequate for Newtonian wastes, it cannot be assumed to be adequate for the full range of wastes expected to be processed at WTP.

For these reasons, evaluation of the design-guide adequacy cannot be complete without an evaluation of predictions of critical velocities in non-Newtonian systems. Unfortunately, the 24590-WTP-GPG-M0058, Rev 0 design guide (Hall 2006) does not place any limitations on solids concentration and chemistry to limit usage to the Newtonian cases. As discussed in previous chapters, the 24590-WTPGPG-M-0058, Rev 0 (Hall 2006) viscosity correlation is actually based on non-Newtonian rheology with solids concentrations up to 20 vol\%. For these reasons, the 24590-WTP-GPG-M-0058, Rev 0 design guide (Hall 2006) can be misapplied to non-Newtonian systems (although such an application may not be intentional).

For non-Newtonian fluids with a yield stress, Equation (3.3) is used to determine the particulate stability of the slurry while flowing (Darby 2000). Figure 8.11 presents the results when calculated with the $95 \%$ particle size and coarse-particle density. Results indicate that all of the non-Newtonian fluids are expected to be stable.

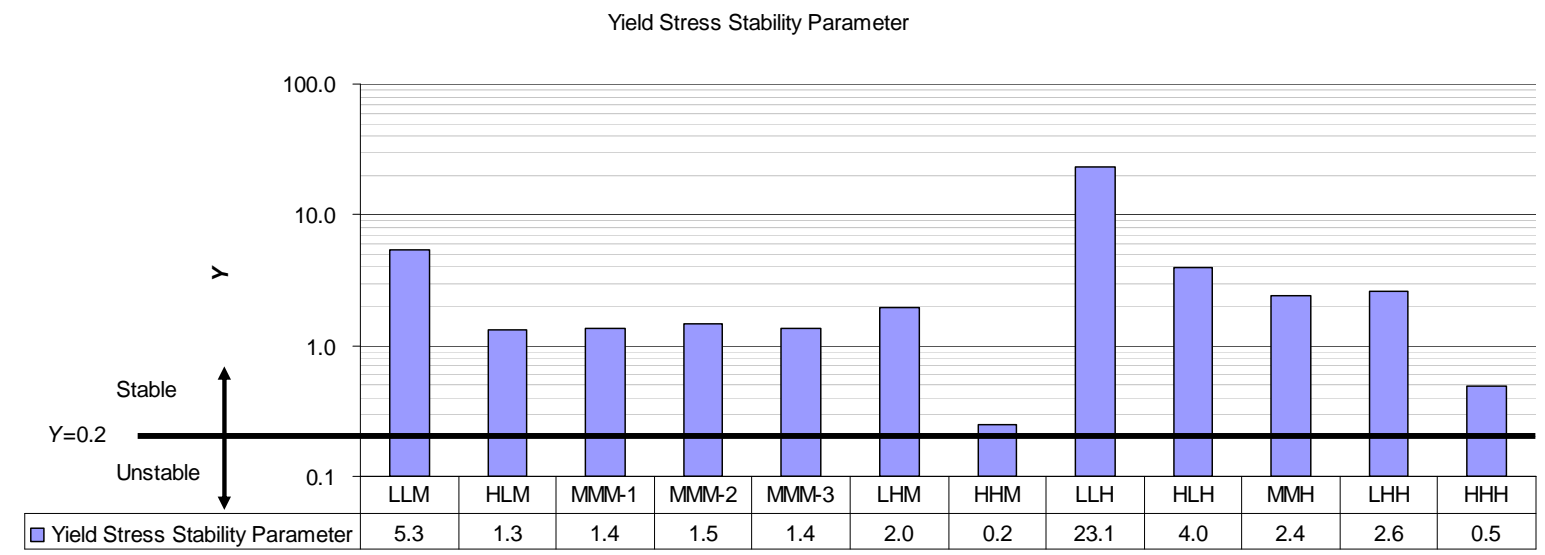

Figure 8.11. Yield-Stress Stability Calculations for non-Newtonian "Medium" and "High" Rheology Systems Using $d_{95}$ Particle Size and Solid Density

Contrary to the prediction of Equation (3.3), Figure 8.12 shows that deposition was experimentally observed for all simulant systems except the LLM and LLH simulants $(10-\mu \mathrm{m}$ glass beads in thin and thick clay). These data show that particles determined to be stable due to yield-stress forces actually settle at moderate flow velocities in the 3 - to $4-\mathrm{ft} / \mathrm{sec}$ range. In addition, the measured deposition velocity of "High" rheology (i.e., thick clay) simulant systems often exceeds the deposition velocity of "Medium" rheology (i.e., thin clay) simulant systems. This gives rise to a non-intuitive observation of increasing the 
rheological properties of a system, resulting in increases to the critical deposition velocity. The observation may be explained by a different physical deposition mechanism than the turbulent eddy transport mechanism that dominates Newtonian slurry systems.

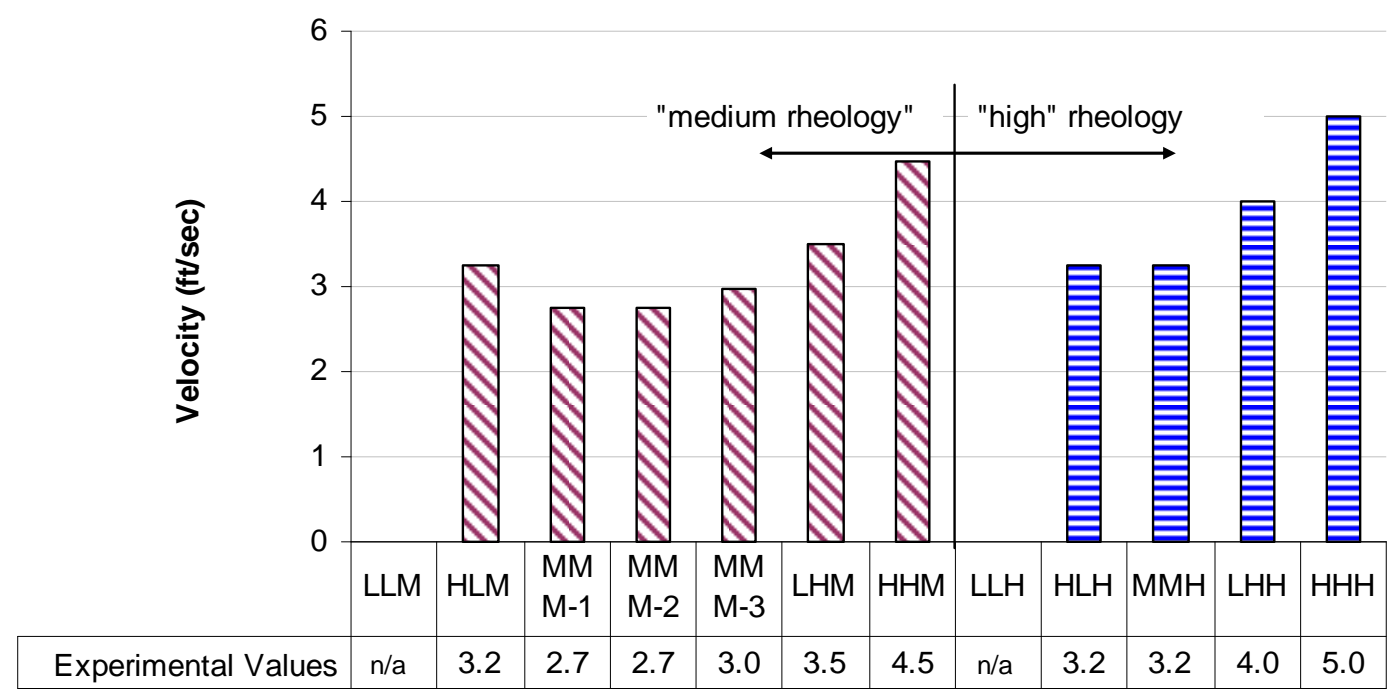

Figure 8.12. Experimentally Measured Values for Critical Velocity of non-Newtonian "Medium" and "High" Rheology Systems

From these data for non-Newtonian systems, the questions posed in Section 3.1 can be answered in the following manner:

1. Role of Density in Calculations - From observations made using the transparent pipe section, the coarse test particles rather than the micron-sized kaolin clay were depositing in the piping systems during these non-Newtonian rheology tests. The design guide specifies the use of the $d_{95}$ density; this term is ambiguous and needs clarification in the design guide. Nonetheless, this value is interpreted to mean the density of the $d_{95}$ particle. This density value is irrelevant for critical velocity calculations. Often this value is unknown, and Equation 1 of the design guide (Hall 2006) will be used for design purposes. This equation calculates an average or composite density of all solids in the slurry. If the average density methodology is applied to the non-Newtonian systems tested in this work, the density of the coarse particles used in critical-velocity calculations is reduced to unrealistically low values. As shown in Figure 8.13, the particle density is reduced by $40 \%$ for the stainless steel systems. 


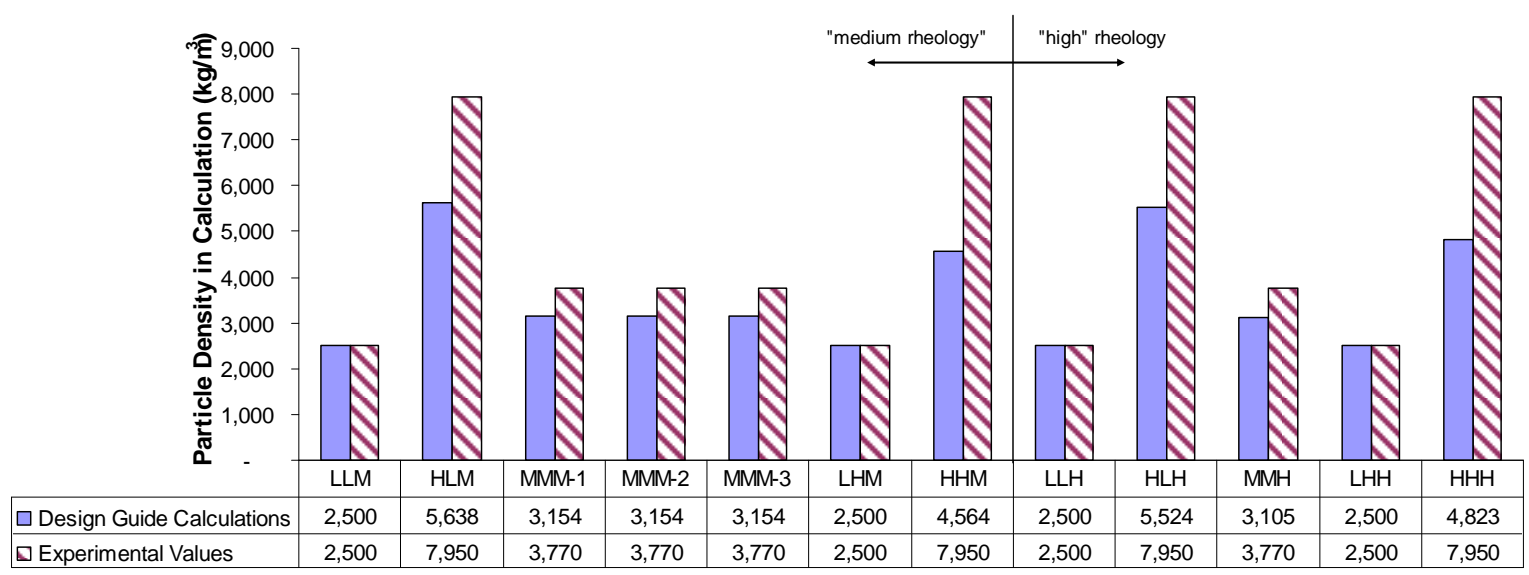

Figure 8.13. Experimental and Corresponding 24590-WTP-GPG-M-0058, Rev 0 Design Guide (Hall 2006) Calculated Values of Particle Density for non-Newtonian "Medium" and "High" Rheology Systems

The RPP-9805 report (Jewett et al. 2002) and the 24590-WTP-GPG-M-0058, Rev 0 design guide (Hall 2006) both use agglomerate densities as their calculation basis. Thomas (1979) studied the role of floc density in critical-velocity experiments, and his conclusions are stated below:

(d) when flocculation tendencies are present [18; the Thomas (1979) equation] will over-predict the deposit velocity because of the lower density of the flocs compared to the discrete particles. Thomas (1962) has previously considered such slurries and used floc size and density measured in static settling tests to develop a deposition criterion. However, it was shown that by using a floc size and density closer to the values of the discrete particle his results are consistent with the present theory. This suggests that the floc size obtained under quiescent conditions is much larger than that present in a turbulent pipe flow, and should not be used to characterize deposition for such flows.

This concept is supported by Jewett et al. (2002) through the following statement:

Although the concept of effective density is easy to describe, it is very difficult to measure in the Hanford Site waste slurries. Some of the difficulties involve the presence of dissolved solids in the interstitial solution and the fragility of the agglomerate structure. The most direct determination of density would involve measuring the mass and volume of the material. However, in the case of these agglomerate particles, measurements of both the mass and volume are confounded by the risk of destroying the structure of the particles when separating them from the surrounding liquid. An attempt to measure the effective density indirectly from settling-rate measurements (Bechtold et al. 2002) was not successful, probably because the particles were broken up by the stirring action used to suspend them.

Considering the fragility of the agglomerates in Hanford waste slurries and the fact that agglomerate structure will vary throughout waste processing, actual rather than effective particle densities should be used for transfer calculations.

Additionally, the composition of the process streams will vary from light elements in the feed streams to heavy elements in the HLW pretreated sludge. Low average bulk particle densities for the Hanford waste feed should not be used as a universal constant for the WTP. The composition of each batch 
and stream should be determined, and appropriate particle-size and density assumptions should be made throughout the WTP process flowsheet.

2. Homogeneous Fraction Concept-As discussed in the Newtonian results section, the slurries that consist of particles below $74 \mu \mathrm{m}$ that were considered to be "homogeneous" in the 24590-WTP-GPGM-0058, Rev 0 design guide (Hall 2006) can be observed settling from the pressure and ERT data. Non-Newtonian results show that settling of these particles occurs even when the yield stability parameter, $Y$, is satisfied (Darby 2000). The reason for this discrepancy is discussed by Shook et al. (2002):

Although Equation 4.3 [the yield stability parameter, Y] is valid in a stagnant fluid, it has been shown to be inappropriate to explain particle suspension when relative motion between the particle and fluid occurs when the fluid is sheared. The minimum particle diameter in these situations is much smaller than that given by Equation 4.3 and reliable methods for predicting $v_{\infty}$ [sedimentation velocities] are still a subject of current research.

Shearing of the fluid lowers the apparent viscosity of the slurry from infinity when stagnant, and it acts as a solid with yield stress to a finite value that typically decreases with increasing shear. For this reason, the use of the stability criterion for Bingham plastic flows should be reassessed. As a predictor of deposition stability during flow and transport of non-Newtonian slurries, the $Y$ stability criterion does not provide acceptable performance.

3. Use of RPP-9805 Viscosity Prediction-The predicted viscosities from the design guide ranged from $3 \mathrm{cP}$ to $93 \mathrm{cP}$. The effective viscosity of each fluid was calculated at deposition with Equation (8.1). The wall shear stress at deposition was calculated from the methods discussed in Appendix A.

$$
\begin{gathered}
\text { Casson Fluid } \rightarrow \quad \mu_{\text {eff }}=\frac{\mu_{C}}{\left(1-\xi^{\frac{1}{2}}\right)^{2}} \\
\text { Bingham Plastic } \rightarrow \quad \mu_{\text {eff }}=\frac{\mu_{B}}{(1-\xi)}
\end{gathered}
$$

where $\mu_{\text {eff }}$ is the fluid effective viscosity (Pa•s), $\xi$ is the dimensionless ratio of fluid yield stress to shear stress at the pipe wall, $\tau_{y} / \tau_{w}, \mu_{B}$ is the Bingham plastic infinite shear viscosity (Pa॰s), and $\mu_{C}$ is the Casson infinite shear viscosity $(\mathrm{Pa} \bullet \mathrm{s})$. For Bingham plastic fluids, the yield stress used to calculate $\xi$ is $\tau_{\mathrm{B}}$. For Casson fluids, the yield stress used to calculate $\xi$ is $\tau_{\mathrm{C}}$. Additional information on the mathematical models used to describe Bingham and Casson fluids can be found In Appendix A

The effective viscosity and the design-guide correlation values are not in agreement. This is shown graphically in Figure 8.14. In some cases, the design-guide correlation value under-predicts the viscosity by a factor of ten. Additionally, for several cases, the viscosity of the "thick" clay is predicted to the lower than the viscosity of the "thin" clay. Use of a lower viscosity than the actual effective viscosity may appear to be conservative. However, we observed that increasing the rheological properties of a system, resulted in increasing the measured critical deposition velocity. This observation may be explained by a different physical deposition mechanism than the turbulent 
eddy transport mechanism that dominates Newtonian slurry systems. In short, the turbulent eddy transport mechanism used as the bases in the 24590-WTP-GPG-M-0058, Rev 0 design guide (Hall 2006) may not be conservative for non-Newtonian systems.

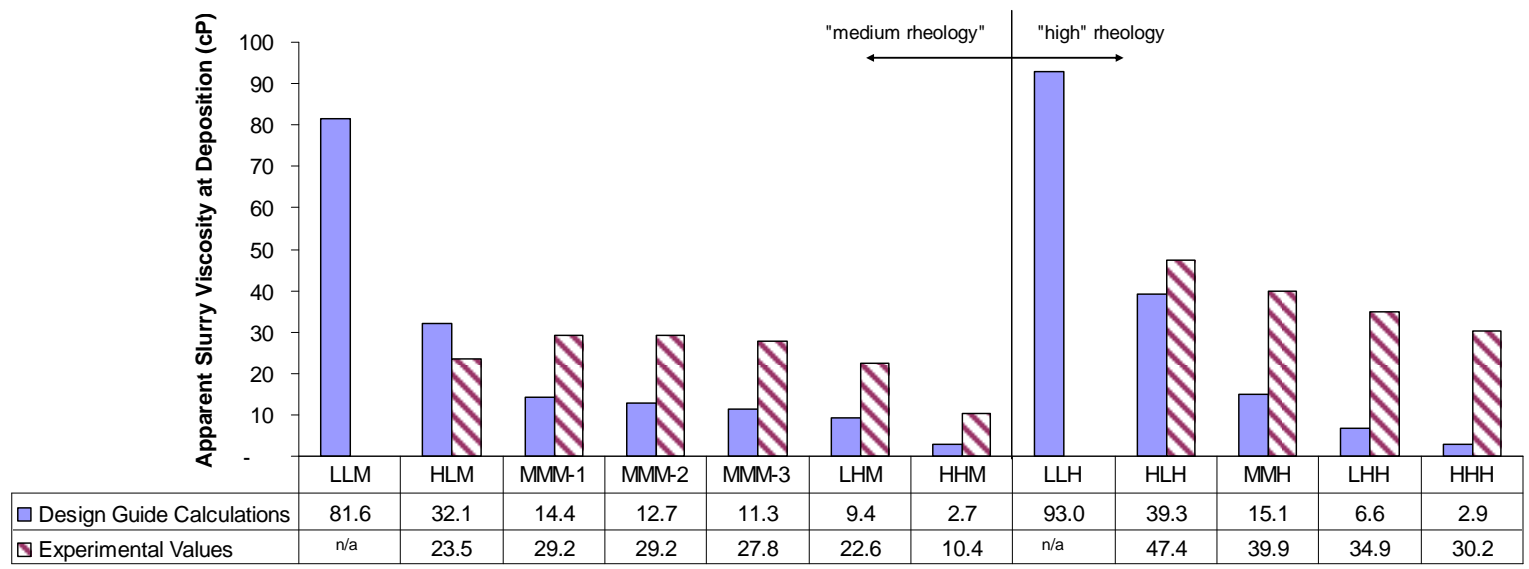

Figure 8.14. Effective and Corresponding 24590-WTP-GPG-M-0058, Rev 0 Design Guide (Hall 2006) Calculated Values of Fluid Viscosity for non-Newtonian "Medium" and "High" Rheology Systems 


\subsection{Recommendations for a non-Newtonian Design Guide}

Based on the limitations described in Section 8.0, the existing 24590-WTP-GPG-M-0058, Rev 0 design guide (Hall 2006) should be modified to incorporate an extended set of design criteria. Specifically, a design guide for critical velocity of both Newtonian and non-Newtonian fluids should consider at least three slurry transport boundary criteria: 1) the critical deposition boundary, 2) the transitional deposition boundary, and 3) the laminar deposition boundary. These boundaries define the transitions between several slurry-transport flow regimes. The transitions correspond to different physical deposition mechanisms. Four of the major regimes are shown schematically in Figure 9.1.

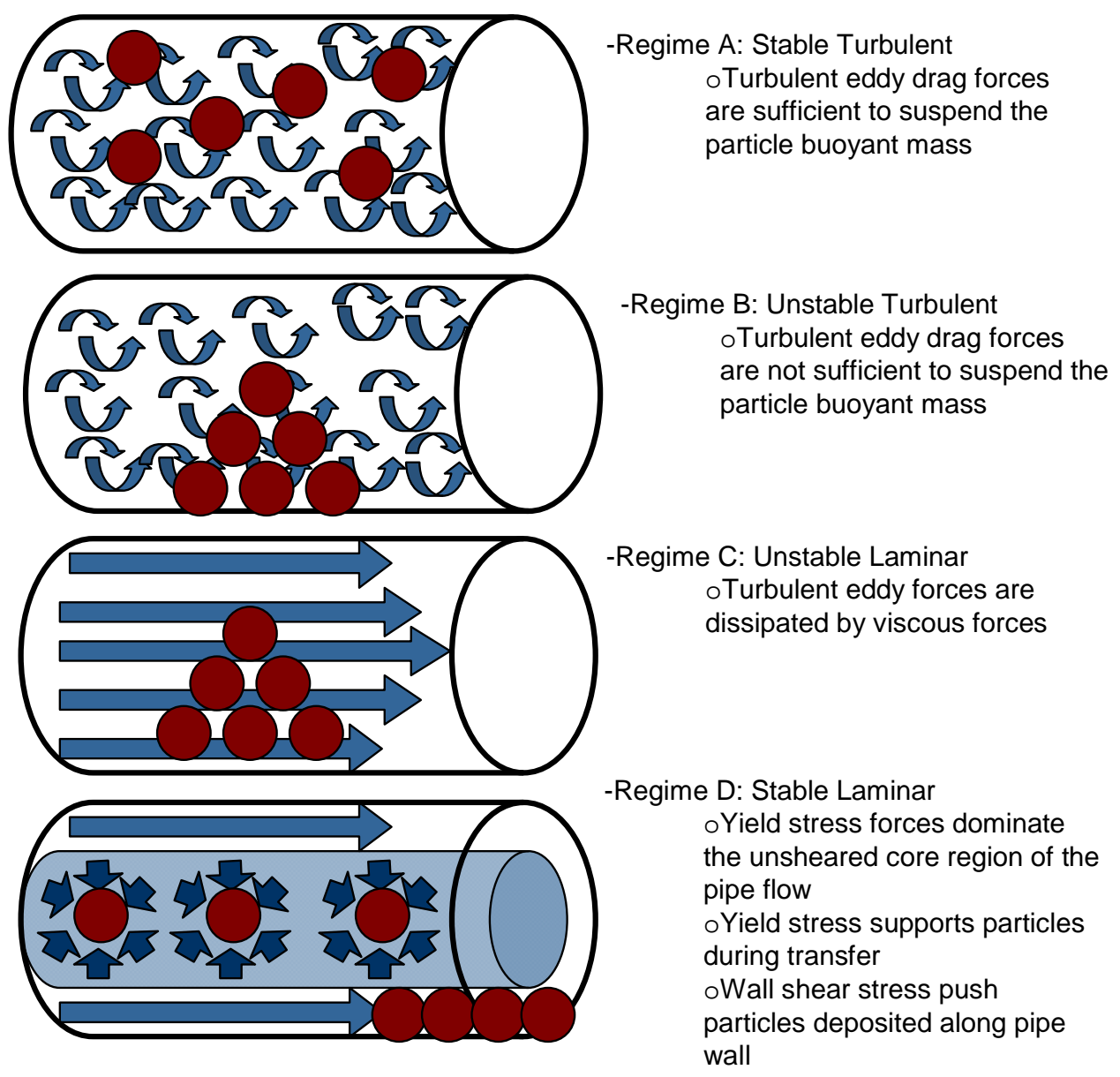

Figure 9.1. Graphical Interpretation of Four Major Slurry-Transport Flow Regimes

The three boundaries listed above are strong functions of slurry rheology. By plotting yield stress on the abscissa and flow velocity on the ordinate, a "stability map" showing these stability regimes can be obtained. An example stability map is shown in Figure 9.2. Point W on the abscissa on Figure 9.2 represents the Newtonian critical deposition velocity for turbulent flow. This point is predicted by the Oroskar and Turian (1980) and Shook et al. (2002) equations. As the non-Newtonian character of the slurry increases with yield stress, the increased apparent viscosity of the slurry results in more drag on the particles and reduces the flow velocity needed to suspend the particles in turbulent flow. The boundary between the stable turbulent and unstable turbulent flow regimes is referred to as the critical deposition boundary and is depicted by path WX on Figure 9.2. 
However, the flow velocity required to reach turbulent flow increases with slurry rheological properties because of viscous forces dampening the formation of turbulent eddies. At point $\mathrm{X}$ in Figure 9.2, the flow becomes dominated by viscous forces rather than turbulent eddies. Since the turbulent eddies necessary for particle transport are not present, the particles will settle when the flow rate crosses this boundary, called the transitional deposition boundary. Along path XY, the stable turbulent and unstable laminar flow regimes are defined.

As the yield stress continues to increase, the required pressure gradient for flow increases. At point $Y$ on Figure 9.2, the required pressure gradient is adequate to push the particles along the pipeline, along the pipeline wall. Often yield stress forces are large enough to suspend the particles in the stagnant core region of a non-Newtonian flow. Along path YZ, the rheological properties continue to increase, which lowers the flow velocity required to achieve the pressure gradient required to push the particles through the pipeline.

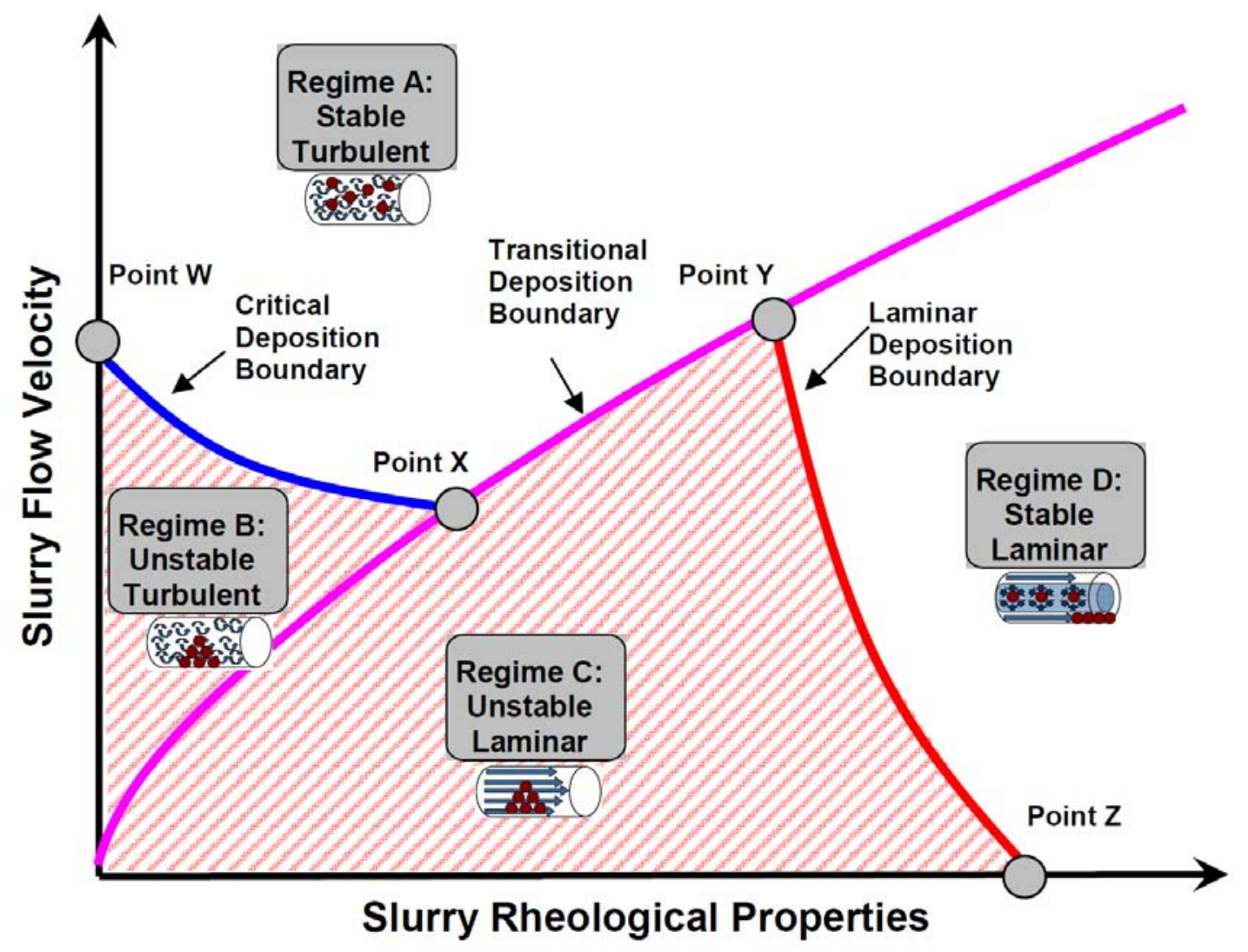

Figure 9.2. Graphical Illustration of a "Stability Map"

\subsection{Critical Deposition Boundary}

The boundary between stable turbulent and unstable turbulent flow can be approximated by calculating the non-dimensional parameters used in the Shook et al. (2002) correlation (see Appendix A). From the experimental Newtonian data in Section 7.0, the plot shown in Figure 9.3 can be obtained. If one assumes a transitional drag coefficient exponent of -0.193, then the Archimedes number exponent is set to 0.15 (see the background information in Appendix A.5). The term $(d / D)^{0.045}$ can be considered to 
be constant. The Shook et al. (2002) limit as the Archimedes Number approaches 80 results in a deposition Froude Number of 1.14. With this relation, the coefficient of the following equation is derived.

$$
F r=0.59 A r^{0.15}
$$

Although the drag exponent of -0.193 provided by Shook et al. (2002) applies to Archimedes numbers between 2760 and 46100, these correlations were for particles settling in a quiescent fluid. In the case of particles settling in a turbulent flow field, one may still expect a transition from the Newton's Law regime to the Stokes' Law regime but the range of Archimedes numbers required for this transition would likely change. Based on the data shown in Figure 9.3, the inference is that this transition occurs around an Archimedes number of 80.

This means that slurry viscosity has a greater effect on particles with a larger surface area-to-mass ratio. The increased viscous forces on these particles result in a decrease in predicted critical velocities from this traditional industry-derived equations that focus on particles larger than $100 \mu \mathrm{m}$ in size. If one applies such equations (i.e., the Oroskar and Turian [1980] correlation from the Hall [2006] design guide) to these particles, a conservative result should be expected. Since the Hanford slurry particles generally have Archimedes numbers less than 200, a design guide based upon the original form of the Oroskar and Turian (1980) correlation with conservative input variables would result in conservative predictions.

As shown in Figure 9.3, the correlation produces a strong correlation coefficient with the Newtonian experimental data with Archimedes Numbers less than 80. Literature data from Parzonka et al. (1981) and Thomas (1979) are also plotted on Figure 9.3 and show similar trends.

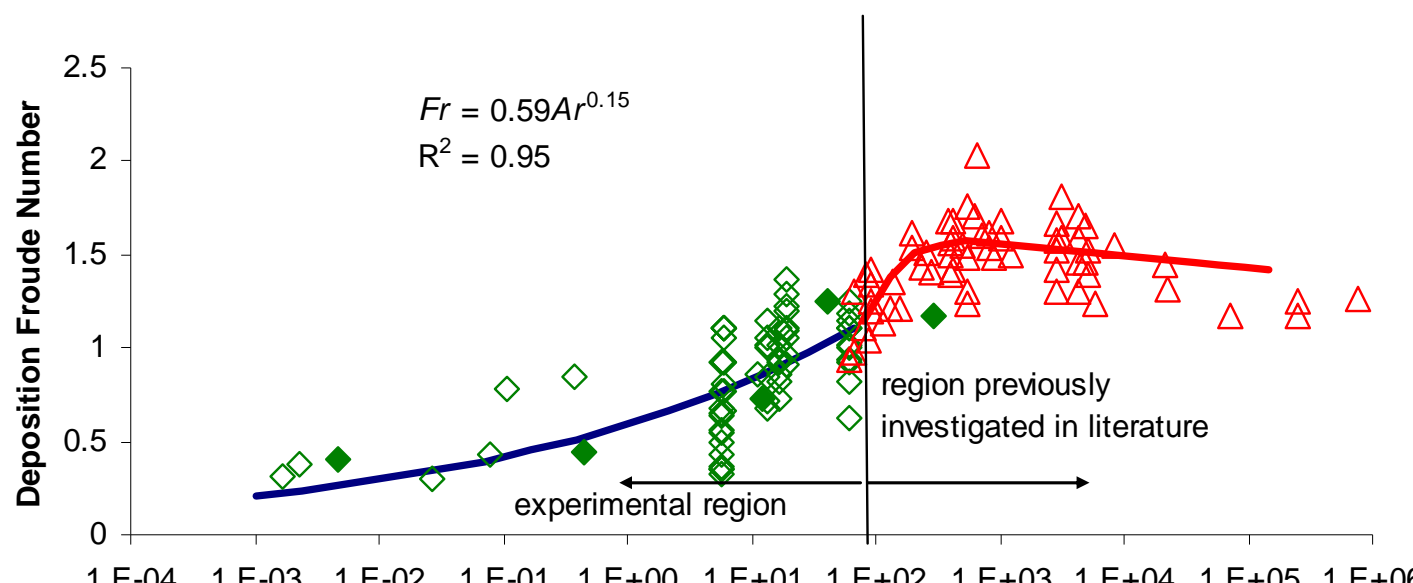

Archimedes Number

\begin{tabular}{ll|}
\hline Gillies et al. (2000) Correlation & $\diamond$ Newtonian Measurements \\
Ar $<80$ correlation & $\diamond$ Parzonka et al. (1981) and Thomas (1979) \\
$\Delta \quad$ Gillies et al. (2000) Data &
\end{tabular}

Figure 9.3. Dimensionless Deposition Froude Number Correlation for Archimedes Number Less than 80 
The data scatter in Figure 9.3 illustrates that for similar systems, a fairly wide range of results may occur. Turian et al. (1987) state that $14 \%$ of the critical-velocity data considered in their article have greater than a 50\% deviation from the predicted values of the theoretical version of the Oroskar and Turian (1980) correlation. Approximately $49 \%$ of the critical-velocity data have a deviation greater than $20 \%$ of the predicted value. The maximum deviation from the theoretical version of the Oroskar and Turian (1980) is reported as 164\%. The empirical version of the Oroskar and Turian (1980) equation that is used in the 24590-WTP-GPG-M-0058, Rev 0 design guide (Hall 2006) may have a better fit to this experimental data set. However, Turian et al. (1987) present statistical data on several alternative empirical forms of the Oroskar and Turian (1980) equation and report that $8 \%$ of the data have a deviation above $50 \%$, and $38 \%$ of the data have a deviation above $20 \%$. These empirical equations have a maximum deviation on the order of $120 \%$. In this context, the 24590-WTP-GPG-M-0058, Rev 0 design guide (Hall 2006) recommendation of a $30 \%$ minimum margin on predictions would account for the majority of the data scatter in the equations. However, to account for engineering margin, an additional increase in the design margin is recommended for application to the WTP. This will help move the design sufficiently above $V_{3}$ and towards $V_{4}$ (see Figure 6.1).

\subsubsection{Extension to Non-Newtonian Systems}

A critical deposition boundary for both Newtonian and non-Newtonian systems is obtained by defining the Archimedes Number used in the Shook et al. (2002) correlations (see Appendix A) supplemented with the $A r<80$ correlation discussed in Section 9.1. The effective viscosity discussed in Section 8.2 is also employed. For Casson and Bingham plastic fluids, the Archimedes Number is defined according to Equation (9.2):

$$
\begin{gathered}
\text { Casson Fluid } \rightarrow \quad A r=\frac{4}{3} \frac{g d^{3}(S-1) \rho_{f}^{2}}{\mu_{C}^{2}}\left(1-\xi^{\frac{1}{2}}\right)^{4}=A r_{\infty}\left(1-\xi^{\frac{1}{2}}\right)^{4} \\
\text { Bingham Plastic } \rightarrow \quad A r=\frac{4}{3} \frac{g d^{3}(S-1) \rho_{f}^{2}}{\mu_{B}^{2}}(1-\xi)^{2}=A r_{\infty}(1-\xi)^{2}
\end{gathered}
$$

This new definition of the Archimedes Number can be substituted into the Shook et al. (2002) correlations to obtain a new correlation for Casson and Bingham plastic fluids as shown by Equations (9.3) and (9.4), respectively. These equations define the critical deposition boundary that separates the stable turbulent flow regime from the unstable turbulent flow regime. The ratio of yield stress to wall shear stress is calculated from the methods discussed in Appendix A. The addition of this term accounts for the shear thinning behavior of the non-Newtonian slurries. For Newtonian slurries, this ratio is zero, and the original Shook et al. (2002) correlations appear. 


$$
\begin{gathered}
\text { Casson Fluid } \\
540<A r \rightarrow F r=1.78 A r_{\infty}{ }^{-0.019}\left(1-\xi^{\frac{1}{2}}\right)^{-0.076} \\
160<A r<540 \rightarrow F r=1.19 A r_{\infty}{ }^{0.045}\left(1-\xi^{\frac{1}{2}}\right)^{0.18} \\
80<A r<160 \rightarrow F r=0.197 A r_{\infty}{ }^{0.4}\left(1-\xi^{\frac{1}{2}}\right)^{1.6} \\
A r<80 \rightarrow F r=0.59 A r_{\infty}{ }^{0.15}\left(1-\xi^{\frac{1}{2}}\right)^{0.6} \\
\text { Bingham } \operatorname{Plastic}^{-0.019}(1-\xi)^{-0.038} \\
540<A r \rightarrow F r=1.78 A r_{\infty}{ }^{0.045}(1-\xi)^{0.09} \\
80<A r<540 \rightarrow F r=1.19 A r_{\infty}{ }^{0.04} \operatorname{Ar}<160 \rightarrow F r=0.197 A r_{\infty}{ }^{0.4}(1-\xi)^{0.8} \\
A r<80 \rightarrow F r=0.59 A r_{\infty}^{0.15}(1-\xi)^{0.3}
\end{gathered}
$$

A non-dimensional plot of the Newtonian and non-Newtonian results discussed in Sections 7.0 and 8.0 is shown as Figure 9.4. With the exception of a single point, HHM, or 100-micron stainless steel in thin clay, all of the non-Newtonian data are above to the left of the turbulent stability correlation (which is applicable to both Newtonian and non-Newtonian slurries). The shift to the left is in response to the increased viscosity of the non-Newtonian carrier slurry, which causes a reduction in the effective Archimedes number of the non-Newtonian slurry. Hence, for non-Newtonian slurries, the turbulent deposition velocity is expected to decrease because of the rise in slurry rheology. However, the actual measured deposition Froude Numbers exceeded the turbulent stability correlation. This indicates that slurry transport operated in a different flow regime for these test cases and is the focus of the discussion in Section 9.2. 


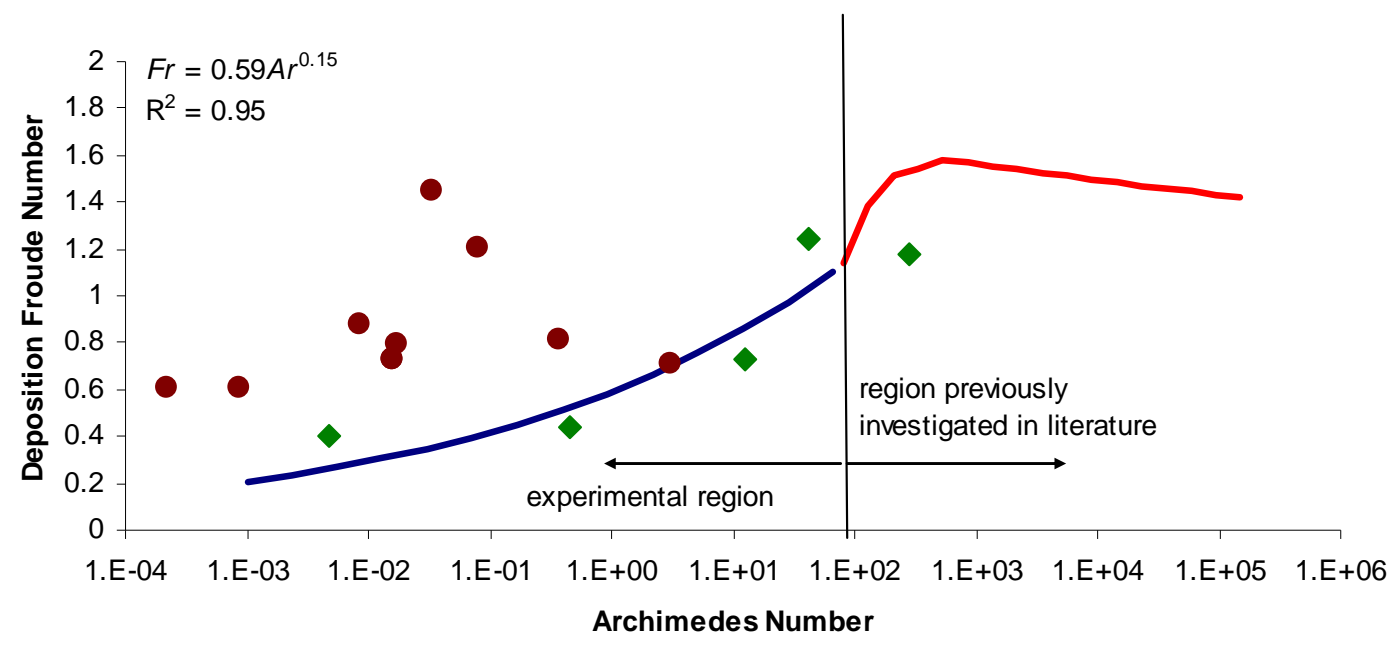

$\begin{array}{lll}\longrightarrow \text { Gillies et al. (2000) Correlation } & \diamond \text { Newtonian Measurements } \\ \operatorname{Ar}<80 \text { correlation } & \bullet \text { non-Newtonian Measurments }\end{array}$

Figure 9.4. Archimedes Number and Deposition Froude Number for both Newtonian and nonNewtonian Data

\subsection{Transitional Deposition Boundary}

If the non-Newtonian Froude-number data (e.g. the data that exist above the correlation shown in Figure 9.4) are plotted against the transition Froude Number, then Figure 9.5 is obtained. The transition Froude Number is defined with the transition velocity from laminar-to-turbulent flow. This plot shows that deposition occurs as the transition velocity from turbulent-to-laminar flow is reached. These data support the statement of Shook et al. (2002) discussed in Section 3.0 that "There have been many experimental investigations of non-Newtonian slurries in laminar and turbulent flow, and deposition is often observed as the laminar flow condition is approached."

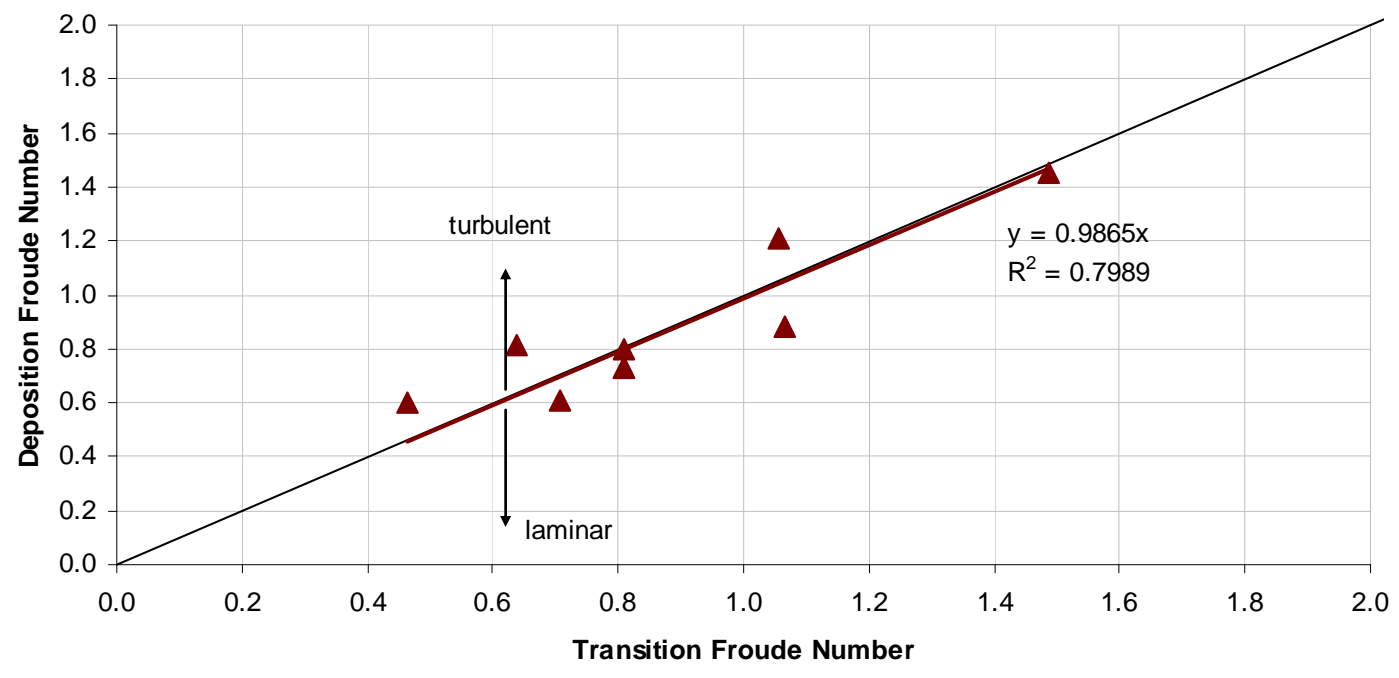

Figure 9.5. Transition and Deposition Froude Number for non-Newtonian Data 
The transition Froude Number can be defined in terms of the Archimedes Number and the transition Reynolds Number according to Equation (9.5).

$$
F r_{t}=\frac{V_{t}}{\sqrt{g D(S-1)}}=\sqrt{\frac{4}{3}}\left(\frac{d}{D}\right)^{\frac{3}{2}} A r_{\infty}^{-\frac{1}{2}} \operatorname{Re}_{t}
$$

The equations below define the transitional deposition boundary that separates the stable turbulent flow regime from the unstable laminar flow regime for both Casson and Bingham Plastic fluids.

$$
\begin{gathered}
\text { Casson Fluid } \rightarrow \quad \operatorname{Re}_{t}=\frac{D V_{t} \rho_{f}}{\mu_{C}}=1050\left[1+\left(1+\frac{C a}{370}\right)^{0.4}\right] \\
\text { Bingham Plastic } \rightarrow \quad \operatorname{Re}_{t}=\frac{D V_{t} \rho_{f}}{\mu_{B}}=1050\left(1+\sqrt{1+\frac{H e}{4500}}\right)
\end{gathered}
$$

The development of Equation (9.6), or a similar version, is supported in several other open literature sources. In a study that examined deposition velocities in non-Newtonian fluids, Shah and Lord (1991) made the following conclusion:

In the present study, most of the critical depositional velocities correspond to the laminar or nearlaminar flow conditions based on the critical value of 2,100 for the Dodge-Metzner (1959) generalized Reynolds number. In this definition of Reynolds number, power-law apparent viscosity is substituted for viscosity in the usual expression for pipe flow.... Generalized Reynolds number values correspond to laminar or transitional flow conditions except for water and low concentrations of HPG [hydroxypropyl guar].

On the subject of deposition occurring in laminar non-Newtonian flows, Gillies et al. (2007) state the following:

The body of evidence suggesting that coarse particle settling can occur during the laminar pipeline transport of mixtures of this type [non-Newtonian slurries with a mixture of fine and coarse particles] is overwhelming. Cooke (2002) and Thomas et al. (2004) provide excellent reviews of such cases where coarse particle settling was observed in operating pipelines and laboratory pipeline loops. In many of these cases, static settling tests show that the mixture is stable - that is, no coarse particle settling occurs. However, when the same mixture is transported by pipeline, coarse particle settling is observed. Recently, Wilson et al. (2003) and Wilson and Horsley (2004) presented a concise and compelling analysis of the fall velocity of particles suspended in nonNewtonian fluids. Their analysis shows unequivocally that the same particle will have a greater fall velocity in a sheared medium than in an unsheared one. There should no longer be any debate about (i) the fact that coarse particle settling can occur in laminar flows and (ii) the absolute irrelevance of static settling tests in determining the tendency of coarse particles to settle during laminar pipeline transport of non-Newtonian mixtures. 
Our results show that the definition of "coarse particles" should include particle density. The only particles not observed to settle were the $10-\mu \mathrm{m}$ glass bead systems in thin and thick clay mixtures. In all other simulant slurries, including those with particle distributions less than $74 \mu \mathrm{m}$, deposition was observed.

Interestingly, line plugging was observed in melter feed transport tests in support of the Hanford Waste Vitrification Plant (HWVP; Gaskill et al. 1996). Glass frit in the size range of 80 to 200 mesh (i.e., 75 to $180 \mu \mathrm{m}$ ) were added to a neutralized current acid waste (NCAW) simulant to form the melter feed. The resulting melter feed was characterized as a Bingham plastic with a yield stress of $25 \mathrm{~Pa}$. A 10-mm glass particle should be suspended in this fluid according to the yield stability parameter, $Y$, discussed by Darby (2000). Consequently, this slurry would be considered homogeneous and non-settling. However, the following observation was made by Gaskill et al. (1996):

As time progressed during the melter feed run, the flow rate would gradually decrease. There are two reasons for this decrease in flow rate. As the melter feed transferred to the melter, the level in the test vessel, HB-15 decreases. With the decreasing level in HB-15, more of the pressure drop across the pump is used to lift the melter feed out of the tank and, therefore, the pump discharge pressure decreases. The flow in the melter feed line decreases as a result of the decrease in pressure in the recycle loop. The second reason for the decreasing flow in the melter feed line is the buildup of material in the melter feed line, thus effectively reducing the flow area and flow rate. Visual evidence of this phenomenon was observed at the end of the nozzle where material was building up and would occasionally fall off or be knocked off. Build-up of solids was also observed in the 3way valve during a visual inspection after the run when the valve was disassembled. It is a safe assumption that build-up occurred at other parts of the system, particularly where there is a changing cross-section, as in the 3-way valve or cross-flow strainer.

The piping system described above was a $3 / 8$-in. pipe with an inner diameter of 0.423 inches. Flow was generally in the 0.20 to 0.35 gallons per minute range. Reynolds numbers under such conditions are extremely low and indicate that the flow was laminar. Again, this substantiates the finding of this report that settling can occur with highly non-Newtonian slurries in laminar flow even when they appear to be non-settling when stagnant.

\subsection{Laminar Deposition Boundary}

Gillies et al. (2007) recently published a paper on the topic of particle transport in laminar, nonNewtonian slurries. The previously discussed "rule of thumb" of the $\sim 1.5-$ to $2-\mathrm{kPa} / \mathrm{m}$ pressure gradient required to transport solids in laminar flow was challenged. A new criterion was established that relates the ratio of the average surficial particle shear stress, $\tau_{\mathrm{p}}$, to the wall shear stress, $\tau_{\mathrm{w}}$. The average surficial particle shear stress (Wilson et al. 2003, 2004) and the ratio are defined by the equations below.

$$
\begin{gathered}
\tau_{p}=\frac{\left(\rho_{s}-\rho_{f}\right) g d}{6} \\
\alpha=\frac{\tau_{w}}{\tau_{p}} \rightarrow \xi=\frac{\tau_{y}}{\alpha \tau_{p}}
\end{gathered}
$$


Gillies et al. (2007) conclude that "a slurry's proclivity to experience laminar flow settling is greatly reduced when $\tau_{\mathrm{w}} / \tau_{\mathrm{p}}>60$ and nearly eliminated when $\tau_{\mathrm{w}} / \tau_{\mathrm{p}}>100 . "$

The laminar flow velocities for these ratios can be obtained analytically for Casson and Bingham plastic fluids as shown by the equations below. These equations define the laminar deposition boundary that separates the unstable laminar flow regime from the stable laminar flow regime.

$$
\begin{gathered}
\text { Casson Fluid } \rightarrow \quad V=\left(\frac{D}{8}\right)\left(\frac{\alpha \tau_{p}}{\mu_{\infty}}\right)\left[1-\frac{16}{7} \sqrt{\frac{\tau_{C}}{\alpha \tau_{p}}}+\frac{4}{3} \frac{\tau_{C}}{\alpha \tau_{p}}-\frac{1}{21}\left(\frac{\tau_{C}}{\alpha \tau_{p}}\right)^{4}\right] \\
\text { Bingham Plastic } \rightarrow \quad V=\left(\frac{D}{8}\right)\left(\frac{\alpha \tau_{p}}{K}\right)\left[1-\frac{4}{3} \frac{\tau_{B}}{\alpha \tau_{p}}+\frac{1}{3}\left(\frac{\tau_{B}}{\alpha \tau_{p}}\right)^{4}\right]
\end{gathered}
$$

\subsection{Stability Maps}

"Stability maps" illustrate the slurry-transport flow regimes discussed in Figure 9.1 that dominate in various modes of operation. For example, Figure 9.2 shows that operating above the critical deposition boundary will result in a stable turbulent regime while operating below this line results in an unstable turbulent flow regime. The area below the transitional deposition boundary results in the unstable laminar regime. Operating to the right of the laminar deposition boundary produces a stable laminar flow regime. Please note that the required $\alpha$ or ratio of average surficial particle shear stress to the wall shear stress for stable laminar flow is not known. Although Gillies et al. (2007) state that $\tau_{\mathrm{w}} / \tau_{\mathrm{p}}>100$ may be adequate for stable laminar flow. For this reason, a range of laminar stability boundaries is presented.

To illustrate these concepts, stability maps were created for each simulant system tested in this work. Using the equations described in Sections 9.1, 9.2, and 9.3 for the critical, transitional, and laminar deposition boundaries, a map of critical velocities as a function of fluid yield stress can be created. The first three points on this map use the physical property and rheological property data in Table 4.4, Table 4.5, and Table 4.6 for the low, medium, and high rheology cases, respectively. Note that the $d_{50}$ particle size value and Bingham plastic rheological properties were used in this analysis. For values exceeding the high rheology value, approximated values of Bingham consistency, yield stress, and slurry density were used. These approximated values were based upon previous rheological property measurements on more highly concentrated kaolin clay/water slurry systems.

Also note that the "limiting critical velocity" or Equation (15) from the 24590-WTP-GPG-M-0058, Rev 0 design guide (Hall 2006) were also calculated and presented for comparison purposes. In cases where $100 \%$ of the particles are smaller than $74 \mu \mathrm{m}$ or particles are considered to be homogeneous due to yield stress forces suspending the particles (Darby 2000), the homogeneous fraction of the slurry can be set to $100 \%$. In such cases, the predicted critical velocity based on Equation (14) from the 24590-WTPGPG-M-0058, Rev 0 design guide (Hall 2006) is reduced to zero, and the "limiting critical velocity" becomes the bounding equation. The measured data and stability maps in Figure 9.6 through Figure 9.10 show that the limiting critical-velocity predictions often fall below the measured experimental values. The limiting critical-velocity predictions also often fall in unstable flow regions on the stability map. For this reason, the limiting critical velocity (Equation 15) from the design guide (Hall 2006) may not be conservative. Furthermore, the value used as the homogeneous fraction governs the critical velocity 
predicted with Equation (14) from the design guide. The criteria for defining the homogeneous fraction in non-Newtonian flows should either be removed or explicitly defined for such systems.

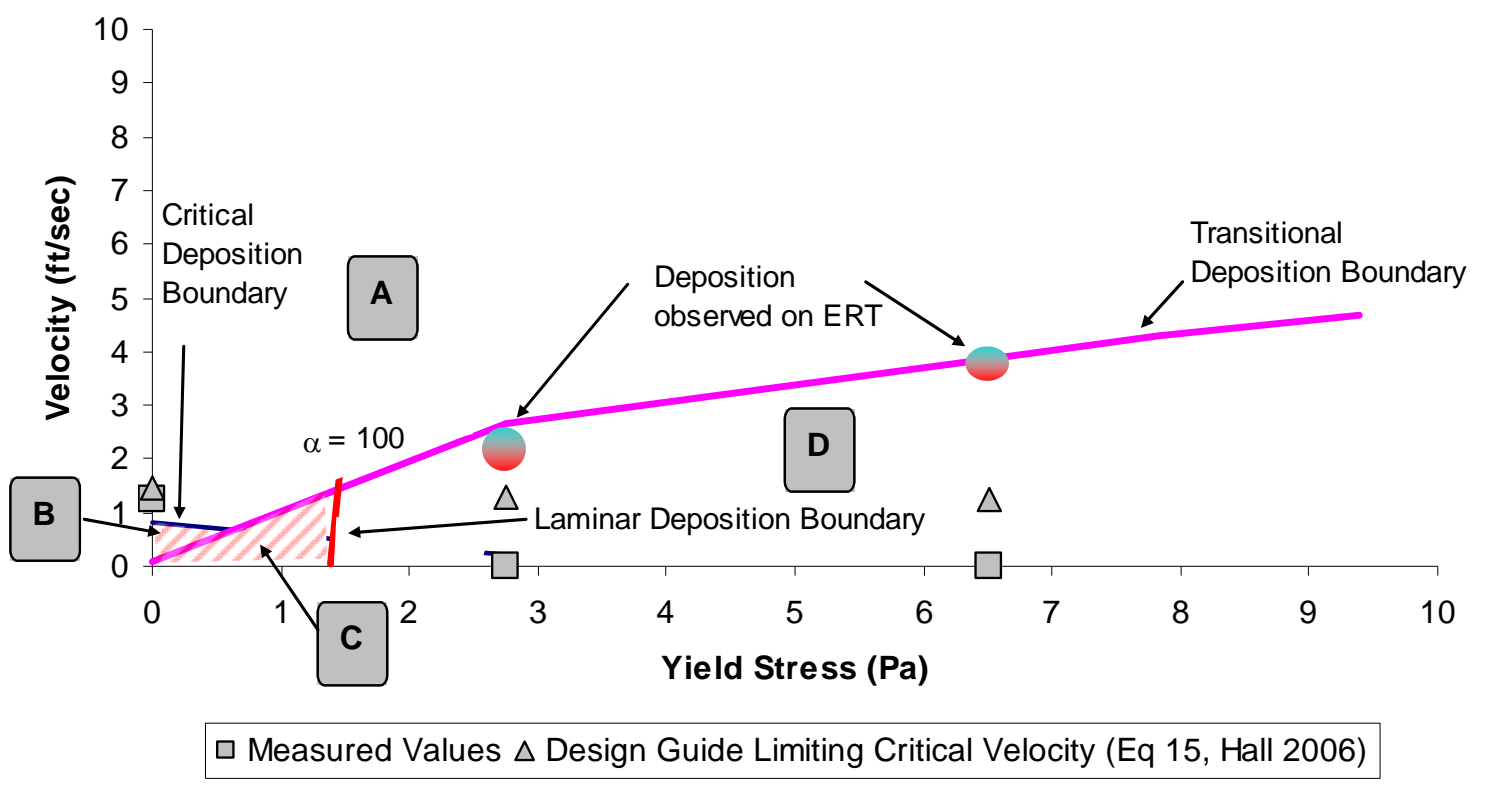

Figure 9.6. Stability Map for LLX Simulant (10- $\mu \mathrm{m}$ glass beads in water, thin clay and thick clay) as a Function of Pipeline Velocity and Yield Stress

Figure 9.6 shows the stability map for the LLX or $10-\mu \mathrm{m}$ glass-bead systems. This map shows that a small unstable transition region is expected to exist between 0.5- and 1.25-Pa yield stress. Experimental results appear to confirm the stability map because at zero yield stress, the system deposits particles near the turbulent stability boundary. Experimental results at LLM and LLH conditions show deposition on the ERT occurring at approximately 2 and $4 \mathrm{ft} / \mathrm{sec}$, respectively. This roughly corresponds to the transition stability boundary. However, a measurable critical velocity is not detected by the pressure transducer data, which is consistent with the behavior of the stable laminar flow regime.

The stability map for the HLX or 10- $\mu$ m stainless steel systems is shown in Figure 9.7. This map shows that the unstable transition region grows when compared to the LLX systems. This is due to the increased surficial particle shear stress for the HLX system. Again, the experimental results appear to confirm the stability map because at zero yield stress, the system deposits particles near the turbulent stability boundary. Experimental results at the HLM and HLH conditions show deposition roughly occurring at the transition stability boundary.

The stability map for the MMX or 50- $\mu \mathrm{m}$ alumina systems is shown in Figure 9.8. Again, this map shows that the unstable transition region grows when compared to the HLX and LLX systems. This is due to the increased surficial particle shear stress for the MMX system. Again, the experimental results appear to confirm the stability map because at zero yield stress, the system deposits particles near the turbulent stability boundary. Experimental results at the MMM and MMH conditions show deposition roughly occurring at the transition stability boundary. 


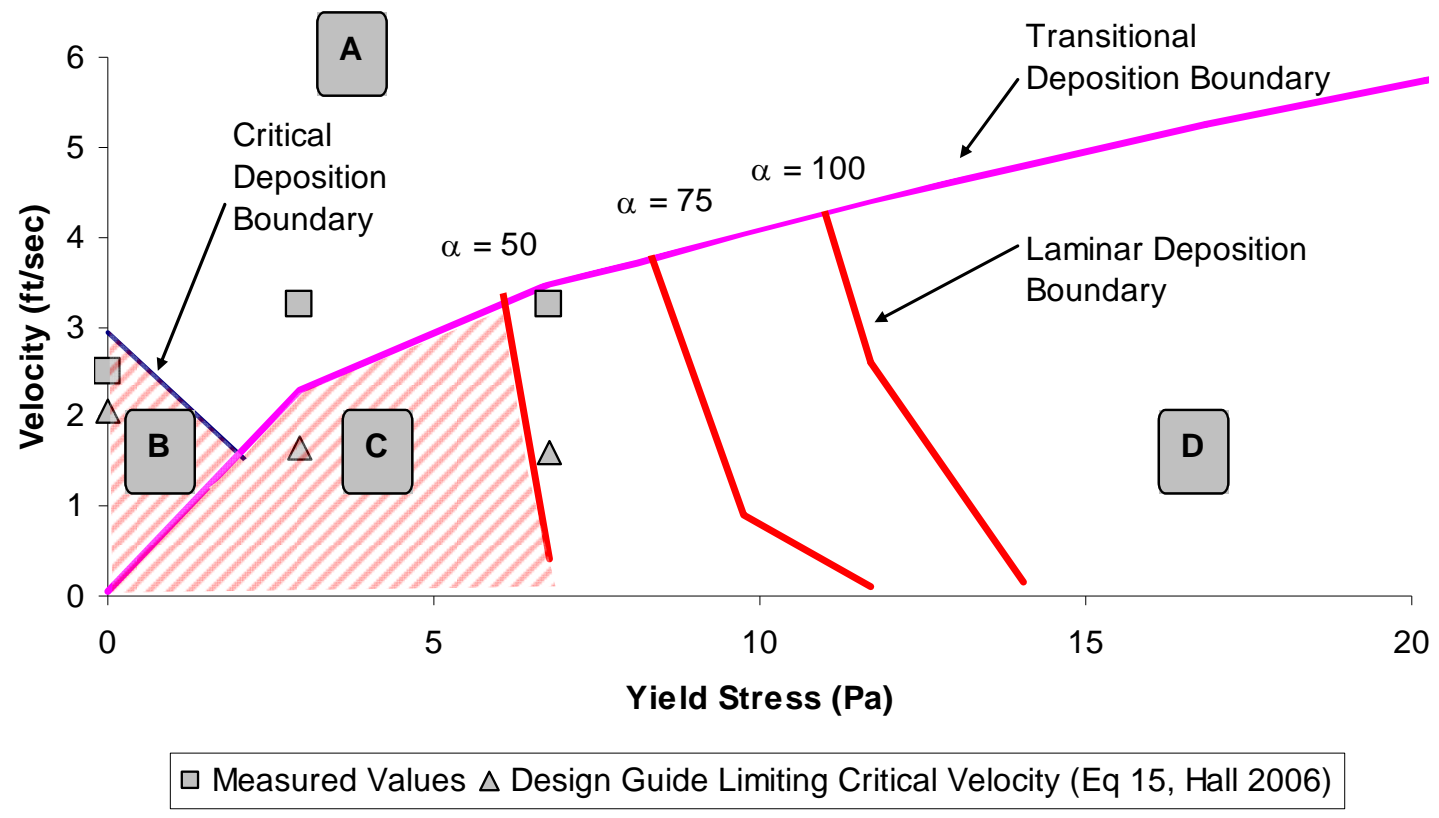

Figure 9.7. Stability Map for HLX Simulant (10 $\mu \mathrm{m}$ stainless steel in water, thin clay and thick clay) as a Function of Pipeline Velocity and Yield Stress

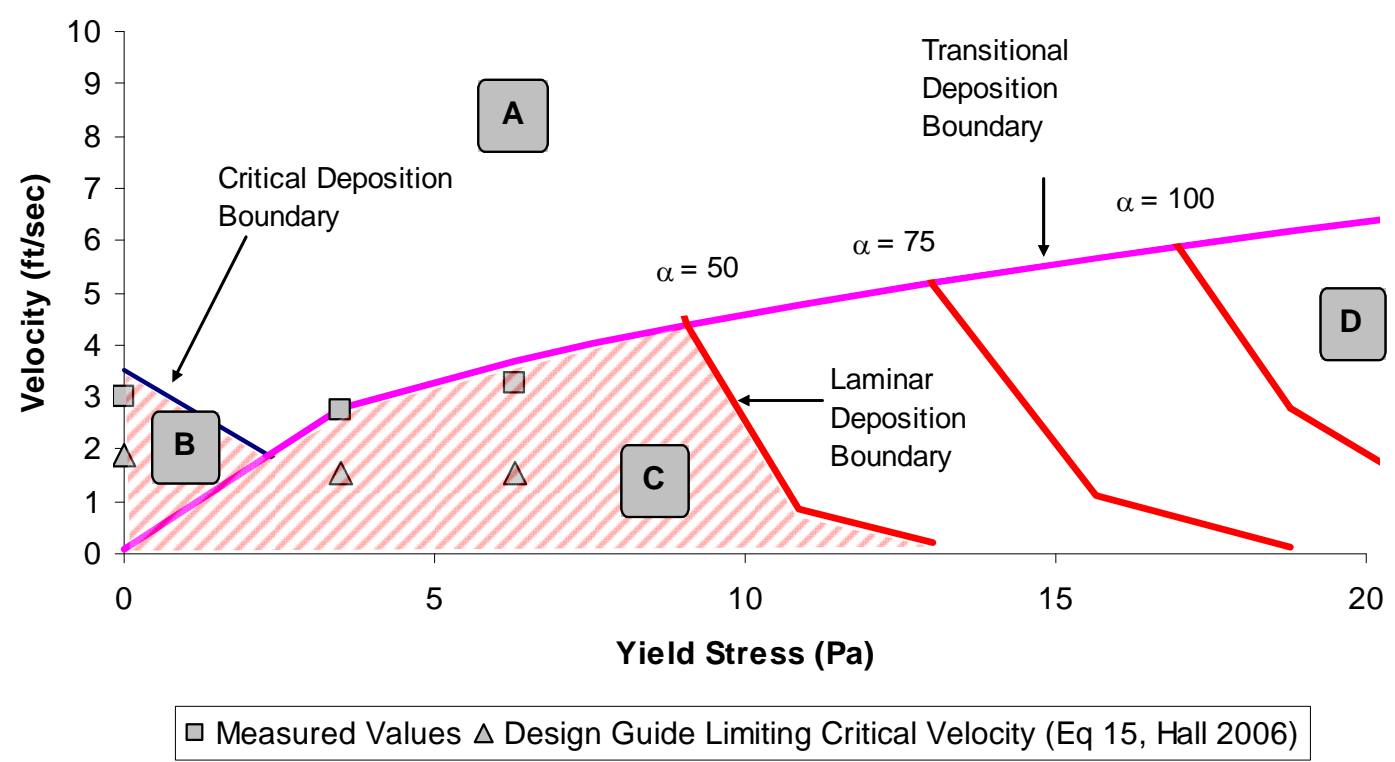

Figure 9.8. Stability Map for MMX Simulant (50- $\mu \mathrm{m}$ alumina in water, thin clay and thick clay) as a Function of Pipeline Velocity and Yield Stress

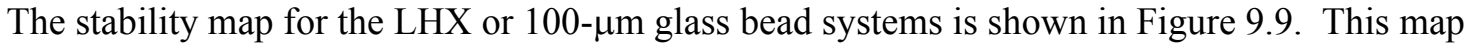
shows that the unstable transition region is smaller when compared to the MMX system. This is due to the decreased surficial particle shear stress for the LHX system. Again, the experimental results appear to confirm the stability map because at zero yield stress, the system deposits particles near the turbulent stability boundary. Experimental results at the LHM and LHH conditions show deposition roughly occurring at the transition stability boundary. 


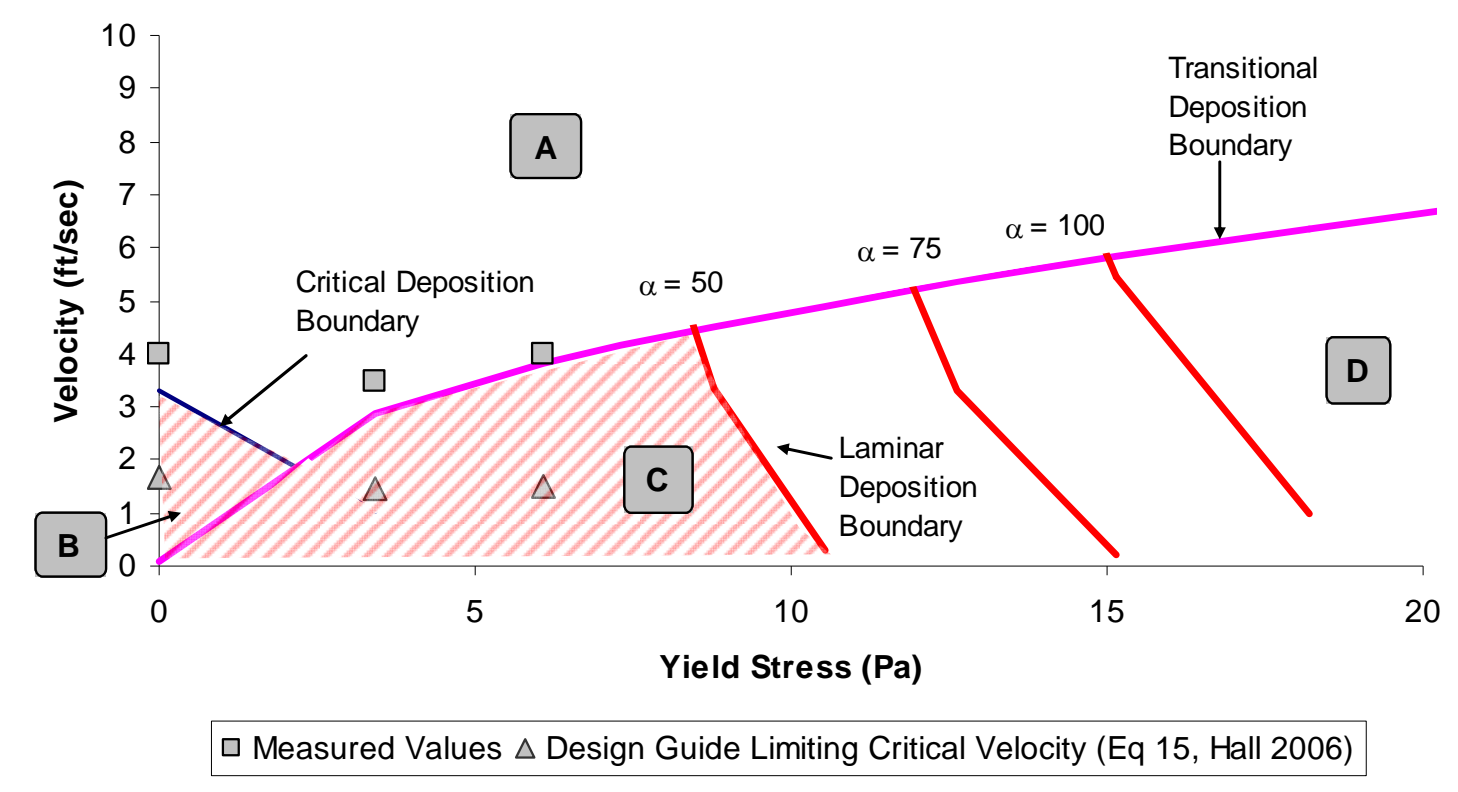

Figure 9.9. Stability Map for LHX Simulant (100- $\mu$ m glass beads in water, thin clay and thick clay) as a Function of Pipeline Velocity and Yield Stress

The stability map for the HHX or $100-\mu \mathrm{m}$ stainless steel systems is shown in Figure 9.10. This map shows the largest unstable transition region when compared to the other systems. This is due to the maximum surficial particle shear stress for the HHX system. Again, the experimental results appear to confirm the stability map because at zero yield stress, the system deposits particles near the turbulent stability boundary. Experimental results from the HHH condition show deposition roughly occurring at the transition stability boundary.

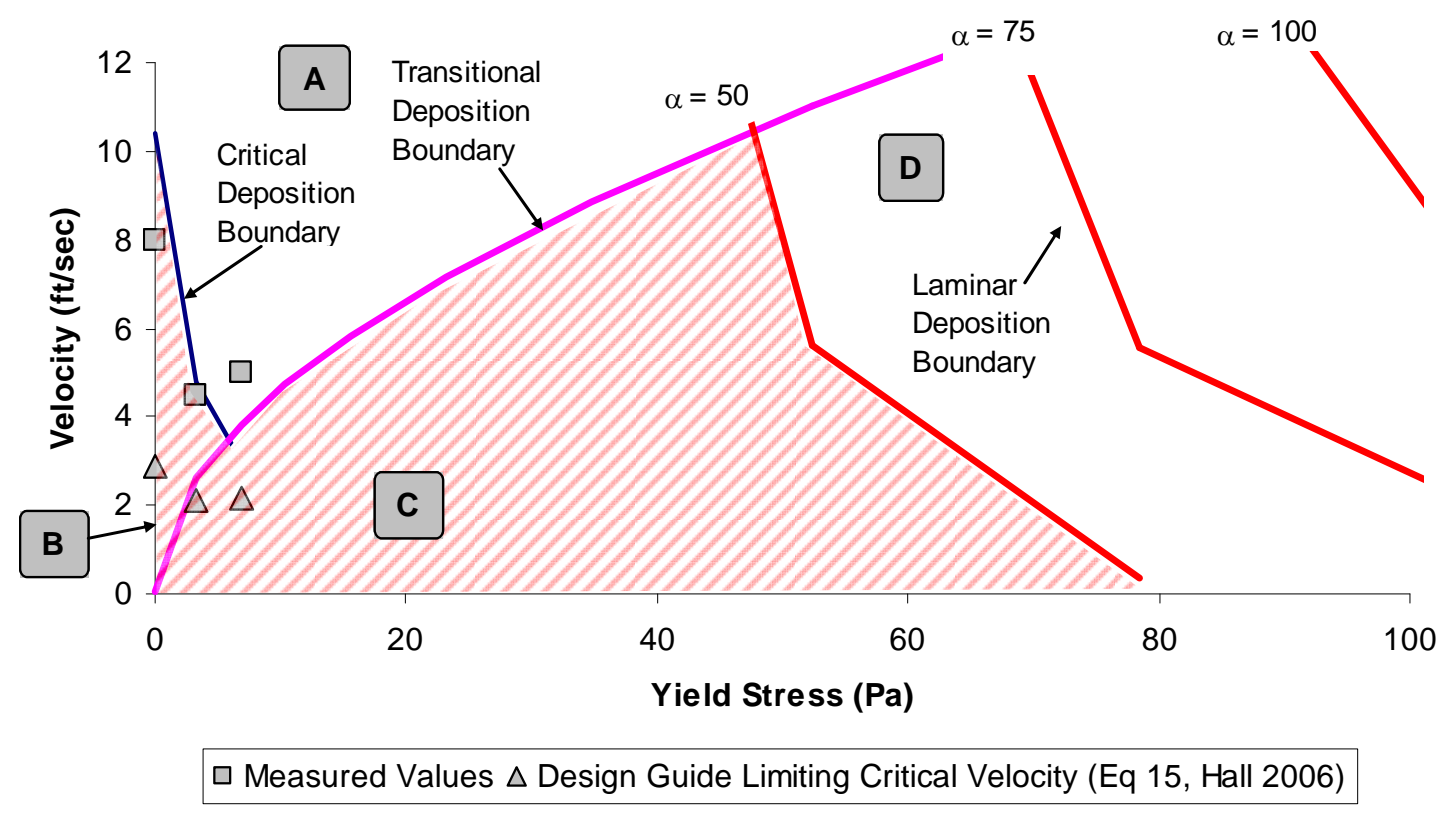

Figure 9.10. Stability Map for HHX Simulant (100- $\mu \mathrm{m}$ stainless steel in water, thin clay and thick clay) as a Function of Pipeline Velocity and Yield Stress 


\subsection{Impact to WTP Process Streams}

With the equations described in Sections 9.1, 9.2, and 9.3 for the turbulent, transition, and laminar stability boundaries, a map of critical velocities as a function of fluid yield stress can be developed for various WTP processing scenarios. For example, $\mathrm{PuO}_{2}$ and $\mathrm{U}_{3} \mathrm{O}_{8}$ particles have been observed in SEM images of various tank slurries (Wells et al. 2007). While the size of these particles is very low, the density of these materials is quite high. If these particles reach the HLW melter feed stream, they will be transported in a non-Newtonian slurry consisting of glass former chemicals (GFCs). The rheological properties of several HLW meter feed streams has been documented by Poloski et al. (2006). A stability map can be created using an empirical rheology model for AZ-101 HLW melter feed and the particle characteristics of the 10- $\mu \mathrm{muO}_{2}$ particle from tank SY-102 shown in the inset of Figure 9.11. The stability map shown in Figure 9.11 indicates that the transport of such particles is predicted to be stable with water flowing at 4 to $6 \mathrm{ft} / \mathrm{sec}$. However, as the rheological properties increase and the yield stress reaches 7 to $15 \mathrm{~Pa}$, turbulence is dampened, and the particles settle at the laminar-to-turbulent flow transition. At 6-ft/sec, transport may still be stable as the pressure gradient in the pipe may be adequate to force the particles along the bottom of the pipe. However, the required ratio of average surficial particle shear stress to the wall shear stress for such operations is unknown.

Similar calculations for LAW melter feed scenarios were performed for olivine and iron oxide particles in fluid rheologically similar to the AZ-102 LAW melter feed (Poloski et al. 2003). Similar to the HLW melter feed scenarios, the transition stability boundary is encountered for fluids with yield stresses of 5 to $10 \mathrm{~Pa}$ when operating in the 4 to $6 \mathrm{ft} / \mathrm{sec}$ range. These stability maps are shown in Figure 9.12 and Figure 9.13.

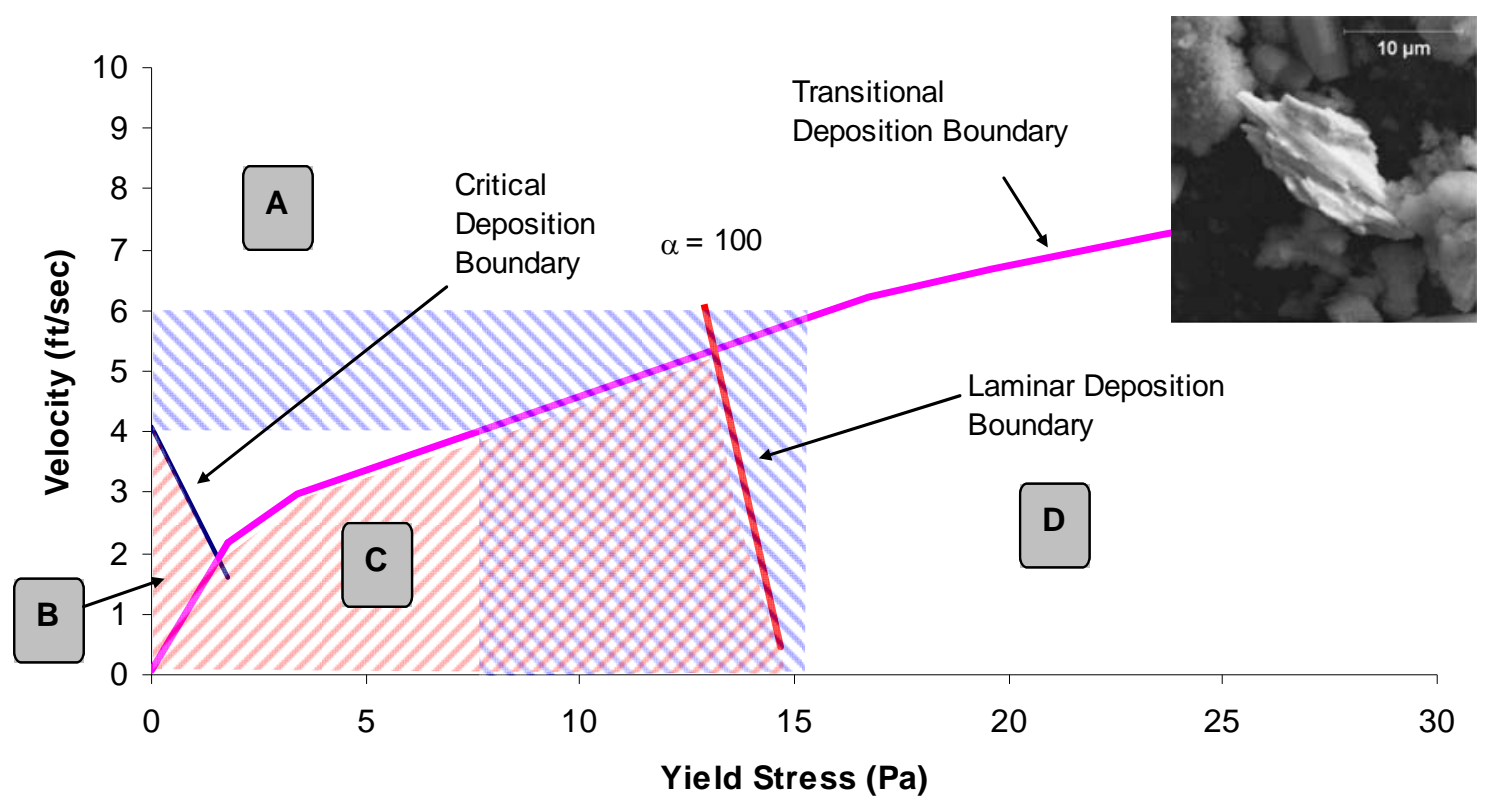

Figure 9.11. Stability Map for $10-\mu \mathrm{muO}_{2}$ Similar to the Inset Image in a Fluid with Rheological Parameters Resembling AZ-101 HLW Melter Feed. $\mathrm{PuO}_{2}$ image taken from SY-102 sample (Wells et al. 2007). 


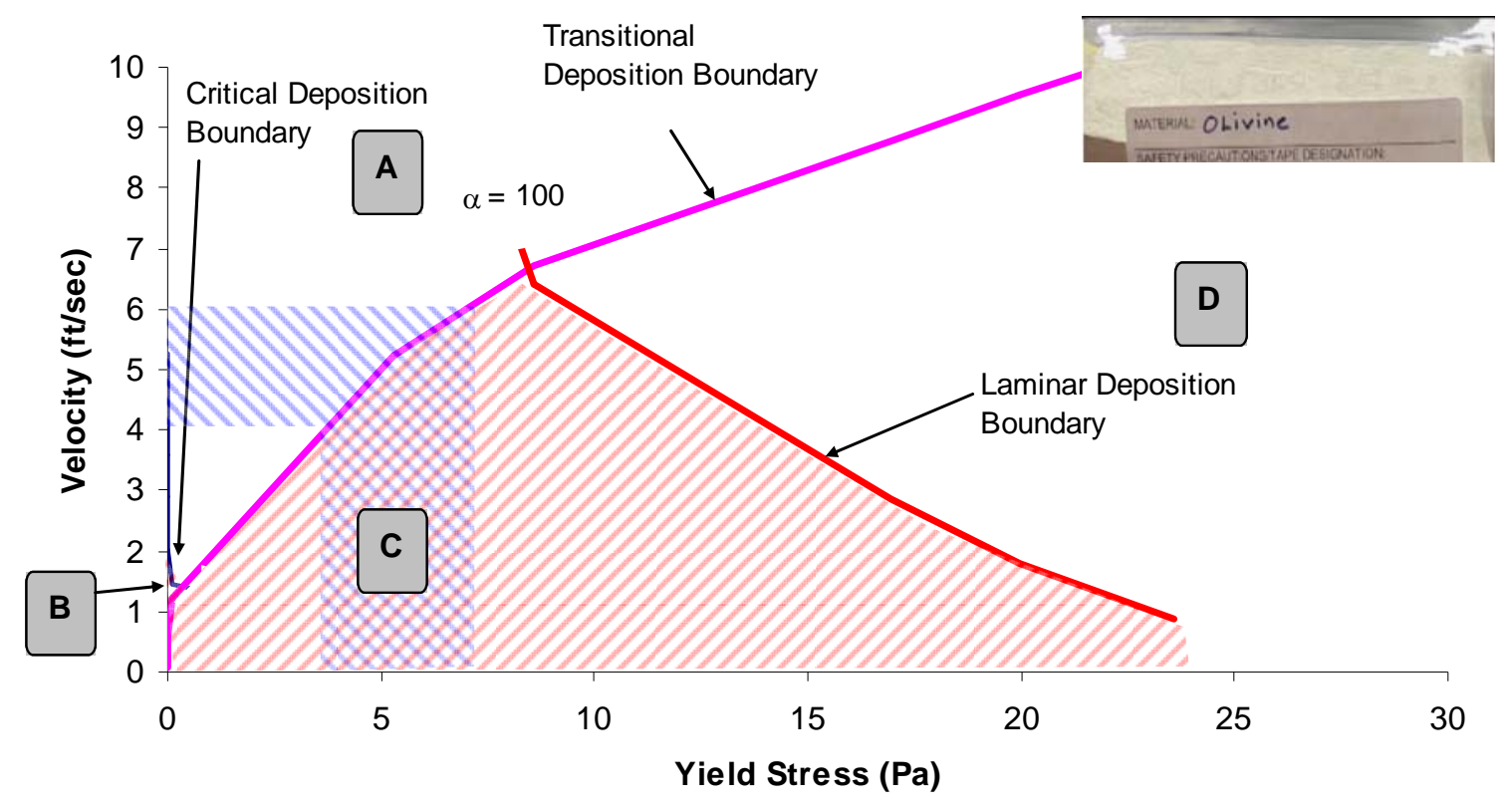

Figure 9.12. Stability Map for $150 \mu \mathrm{m}$ Olivine Similar to the Inset Image of LAW GFC Powder in a Fluid with Rheological Parameters Resembling AZ-102 LAW Melter Feed

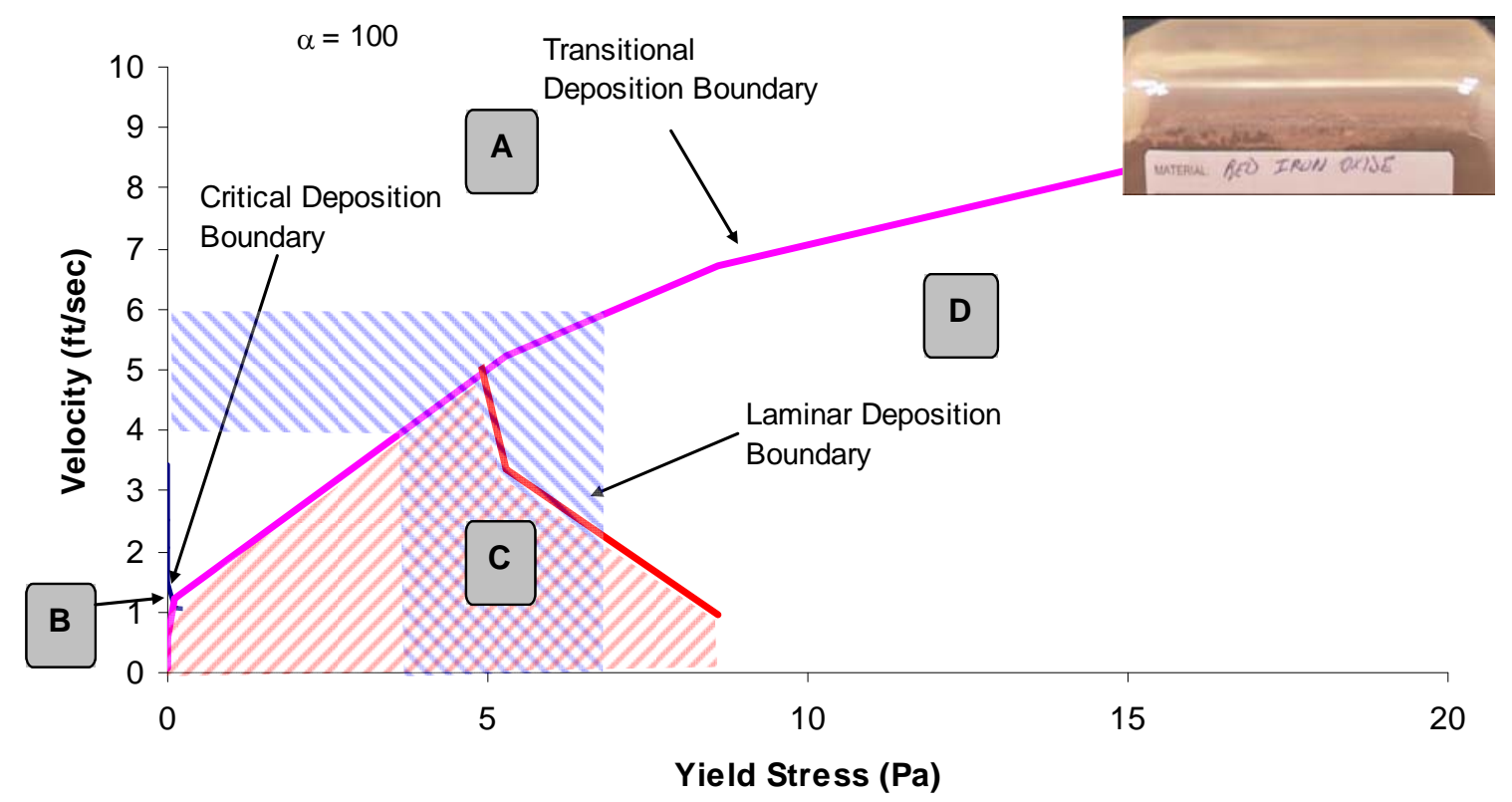

Figure 9.13. Stability Map for $25 \mu \mathrm{m} \mathrm{Fe}_{2} \mathrm{O}_{3}$ Similar to the Inset Image of LAW GFC Powder in a Fluid with Rheological Parameters Resembling AZ-102 LAW Melter Feed 


\subsection{Flush-System Testing}

The 24590-WTP-GPG-M-0058, Rev 0 design guide (Hall 2006) specifies upper limits on the flushing velocity at $12 \mathrm{ft} / \mathrm{sec}$ and $10 \mathrm{ft} / \mathrm{sec}$ for the process streams without and with GFCs, respectively. This upper limit is in place to limit pipe erosion. The flush volume upper limit is stated at 3 line volumes. The lower flush volume limit is 1.5 and 1.7 line volumes for Newtonian and non-Newtonian process lines, respectively. The experimental flow loop was determined to have approximately 60 gallons of line volume. This section discusses how the flush was performed with respect to the 24590-WTP-GPG-M0058, Rev 0 design guide (Hall 2006) constraints.

\subsection{Flush-System Results}

Following the procedure discussed in Section 6.0, a high pressure flush was conducted after each velocity ramp down. The initial charge pressure for line flushes was set between 100 and $110 \mathrm{psig}$. The flush system was opened, and the data-acquisition system recorded pressures, flow rates, and slurry density.

The level in the flush tank was recorded and converted to a volume from a calibration curve. The ratio of the cumulative flush tank volume discharge to the 60 -gallon loop volume is used as the flush-to-line volume ratio.

The reported pressure is the pressure across the entire flow loop measured via differential pressure transducer port 3. The reported velocities are from the loop entrance Coriolis flow meter. If the settled solids in the system form a stationary plug of material, water should flow through that plug slowly, providing a near zero velocity reading on the system flow meters while the flush tank level drops.

The reported density is taken from the outlet Coriolis flow meter. When the measured density is high, the flush is still removing particles from the system. As the density approaches the value of the flush water, the effectiveness of the flush is nearing completion. Verification that the line is indeed clean involved observing a residual amount of solids present in the transparent section of piping.

A target flush volume of 1.5 to 2 line volumes was selected, and multiple flushes were performed. This results in a total flush-to-line volume ratio typically between 5 and 6 . After this point, the system was purged with compressed air. This is reflected by the abrupt drop in instrumentation signal at the end of several of the plots. The data from these flushes is shown for all 15 simulant systems in Figure 10.1 through Figure 10.15. 


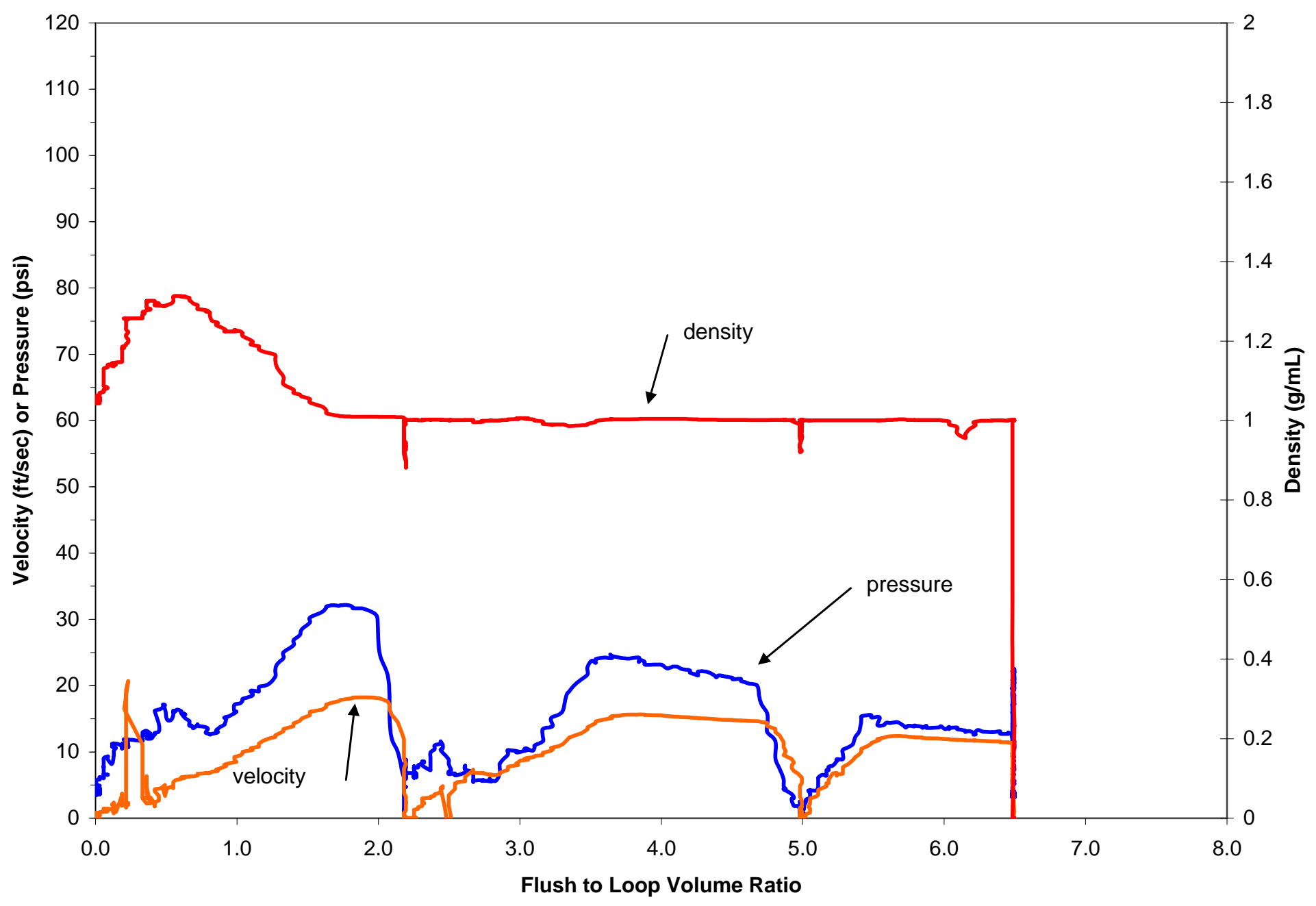

Figure 10.1. Flush Data for LLL Simulant (10- $\mu \mathrm{m}$ glass beads in water) as a Flush-to-Pipe Loop Volume Ratio Showing Three Complete Flushes 


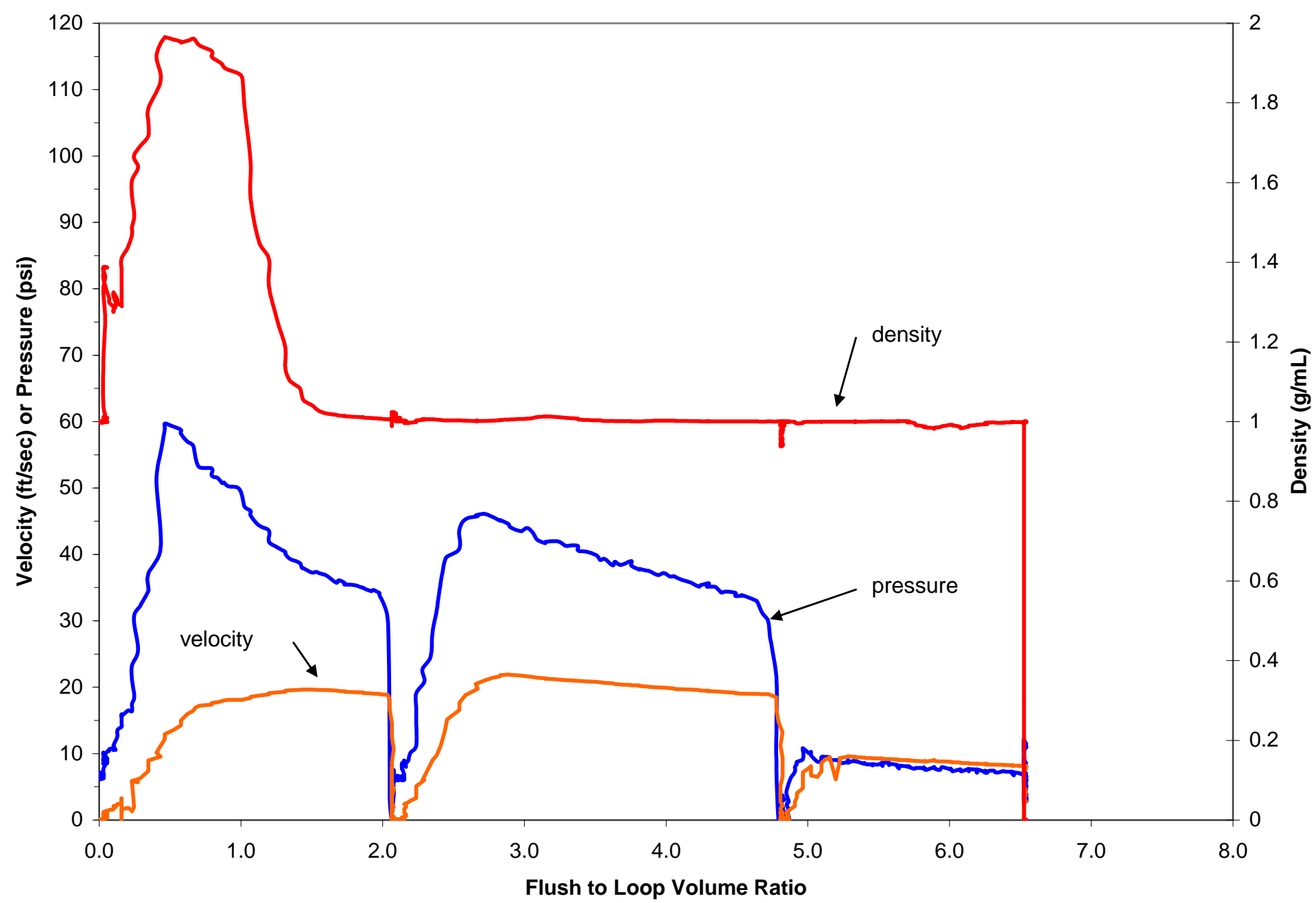

Figure 10.2. Flush Data for HLL Simulant (10- $\mu \mathrm{m}$ stainless steel in water) as a Flush-to-Pipe Loop Volume Ratio Showing Three Complete Flushes 


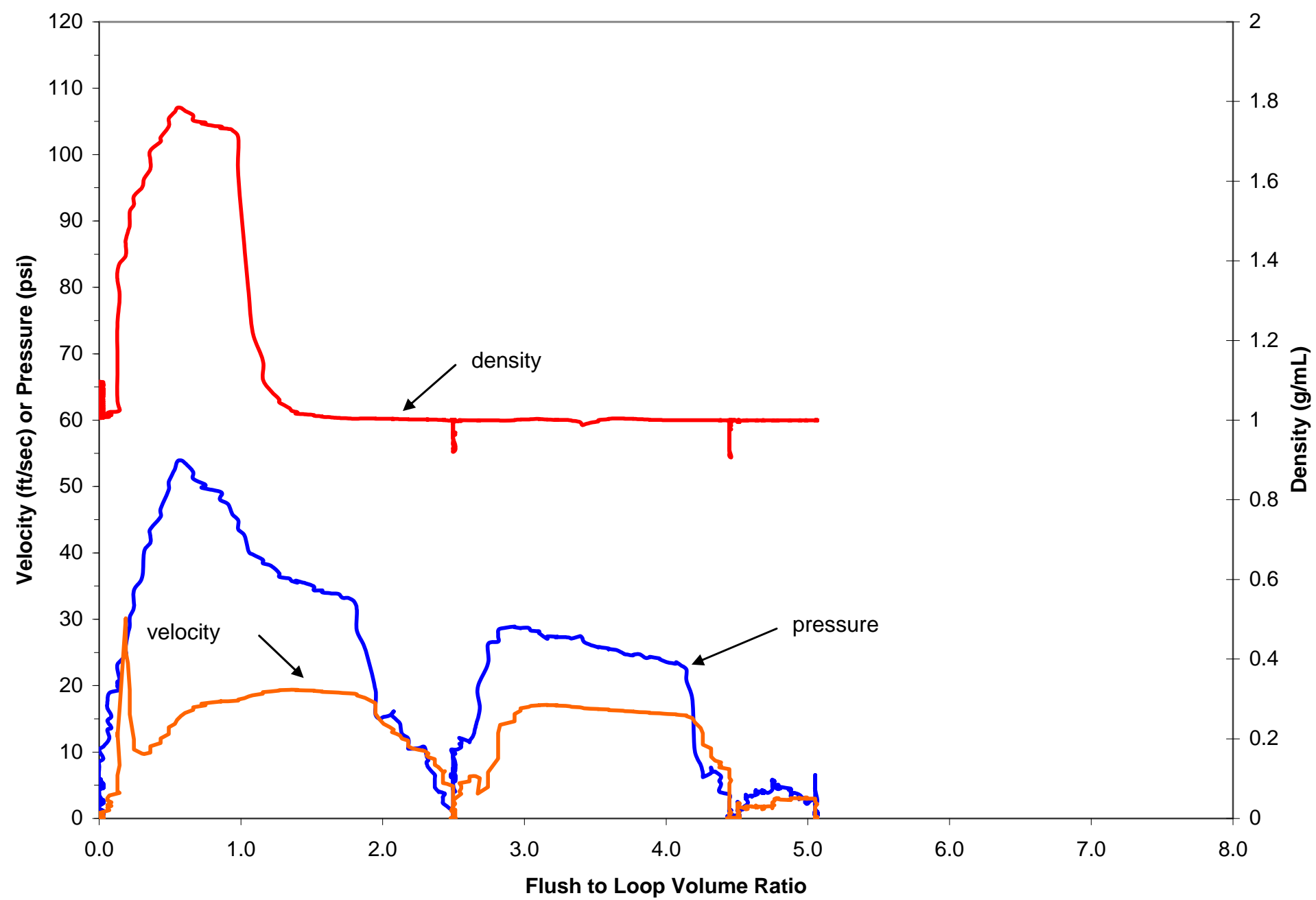

Figure 10.3. Flush Data for MML Simulant (50- $\mu \mathrm{m}$ alumina in water) as a Flush-to-Pipe Loop Volume Ratio Showing Two Complete Flushes 


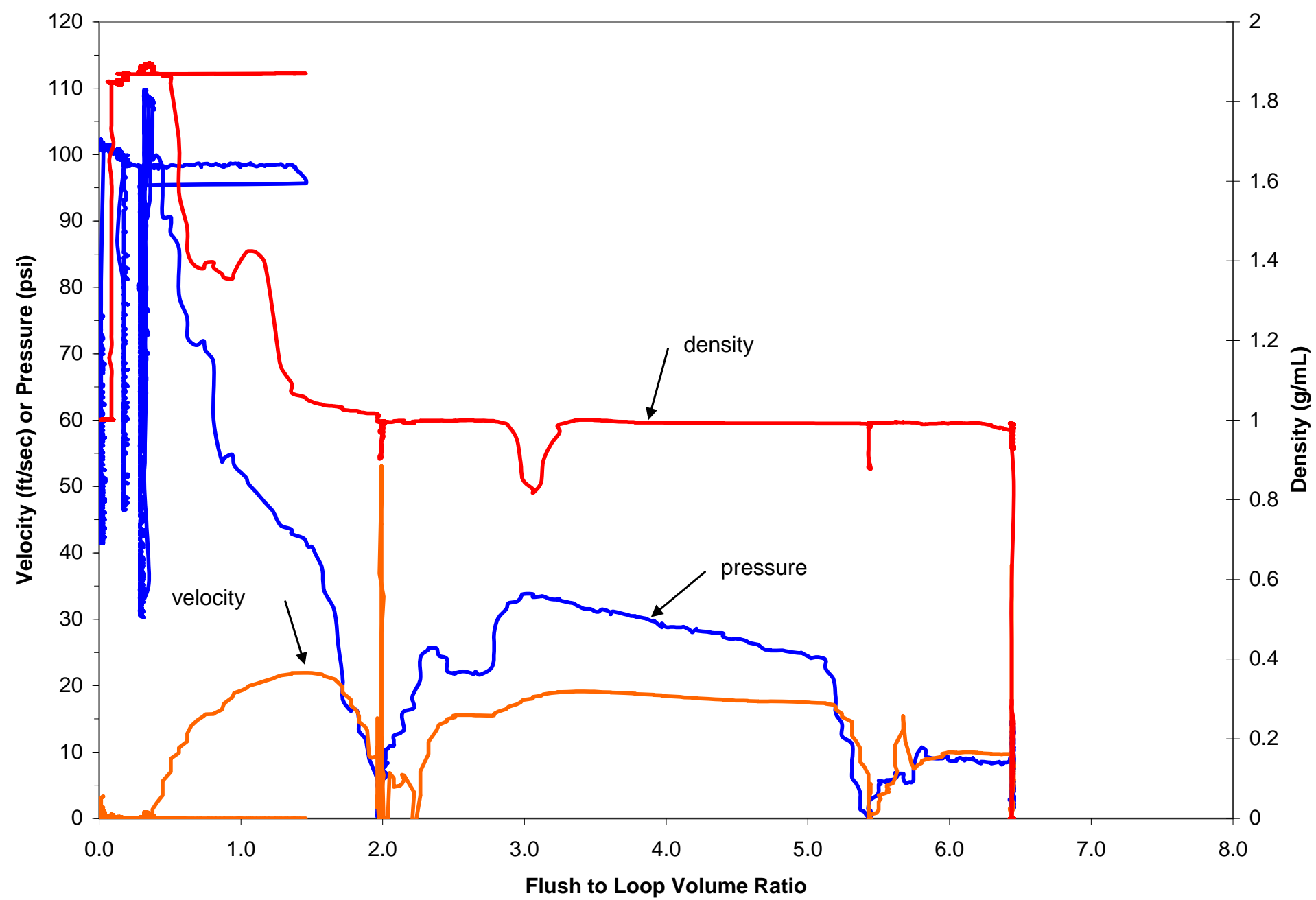

Figure 10.4. Flush Data for LHL Simulant (100- $\mu \mathrm{m}$ glass beads in water) as a Flush-to-Pipe Loop Volume Ratio Showing Three Complete Flushes 


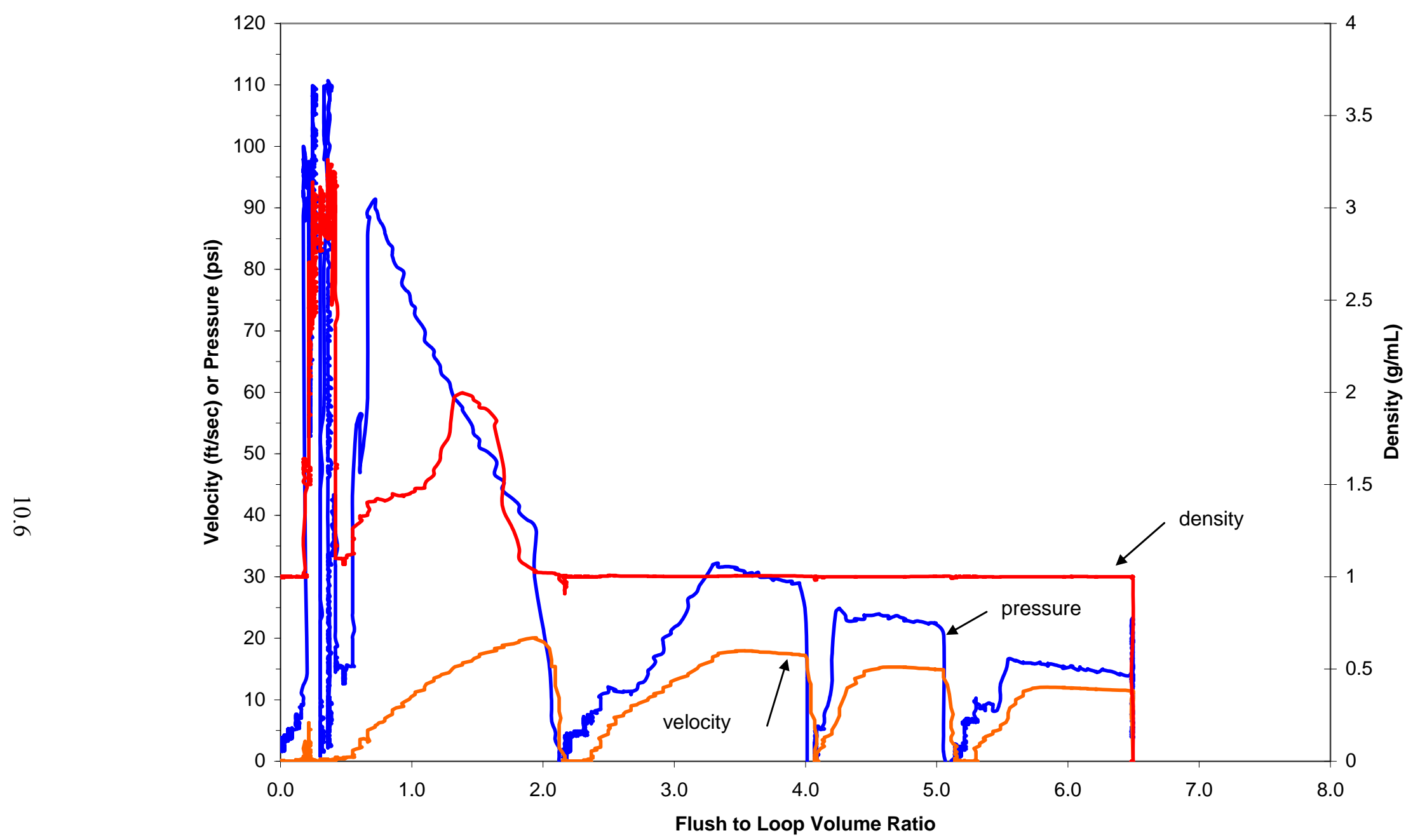

Figure 10.5. Flush Data for HHL Simulant (100- $\mu \mathrm{m}$ stainless steel in water) as a Flush-to-Pipe Loop Volume Ratio Showing Four Complete Flushes 


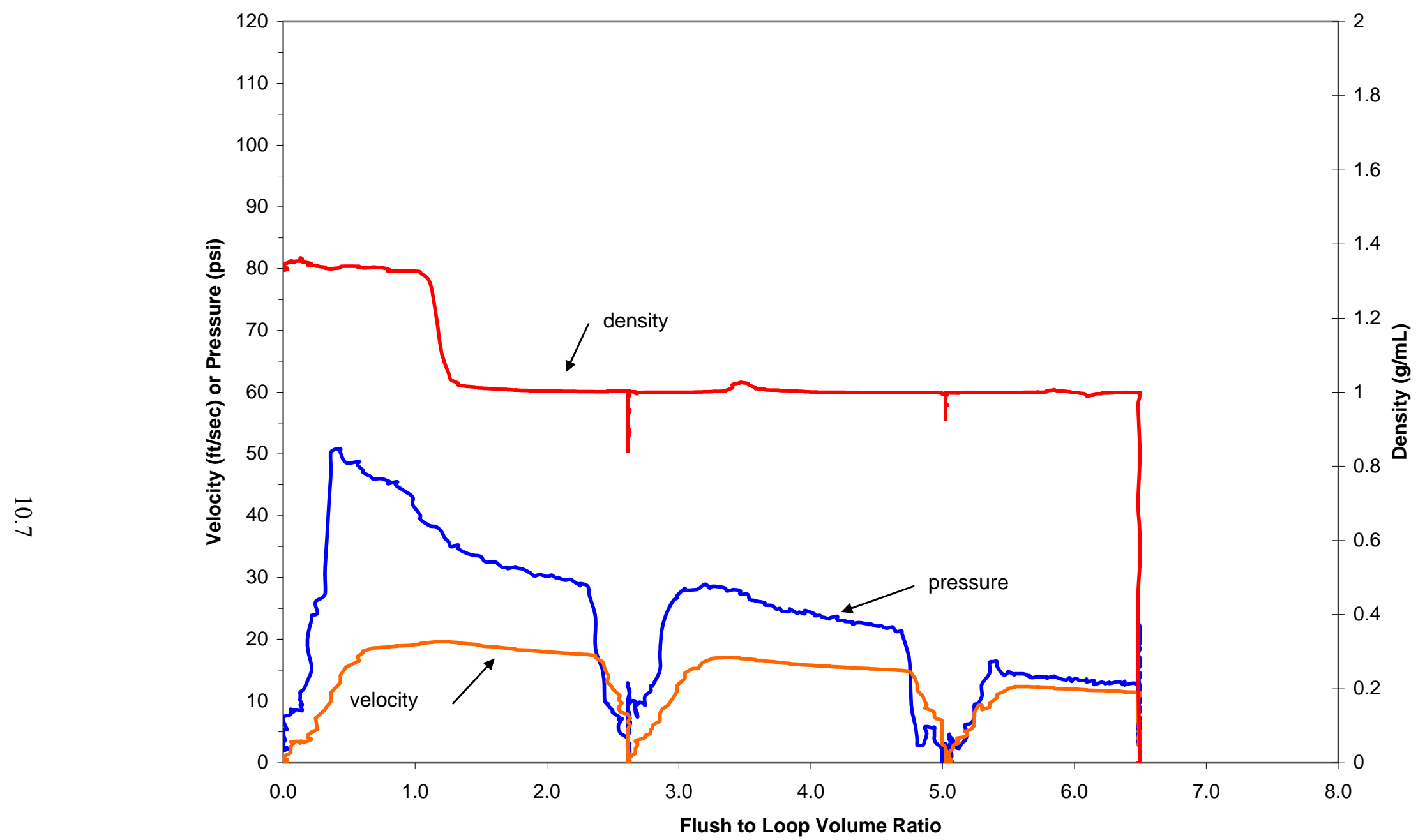

Figure 10.6. Flush Data for LLM Simulant (10- $\mu \mathrm{m}$ glass beads in thin clay) as a Flush-to-Pipe Loop Volume Ratio Showing Three Complete Flushes 


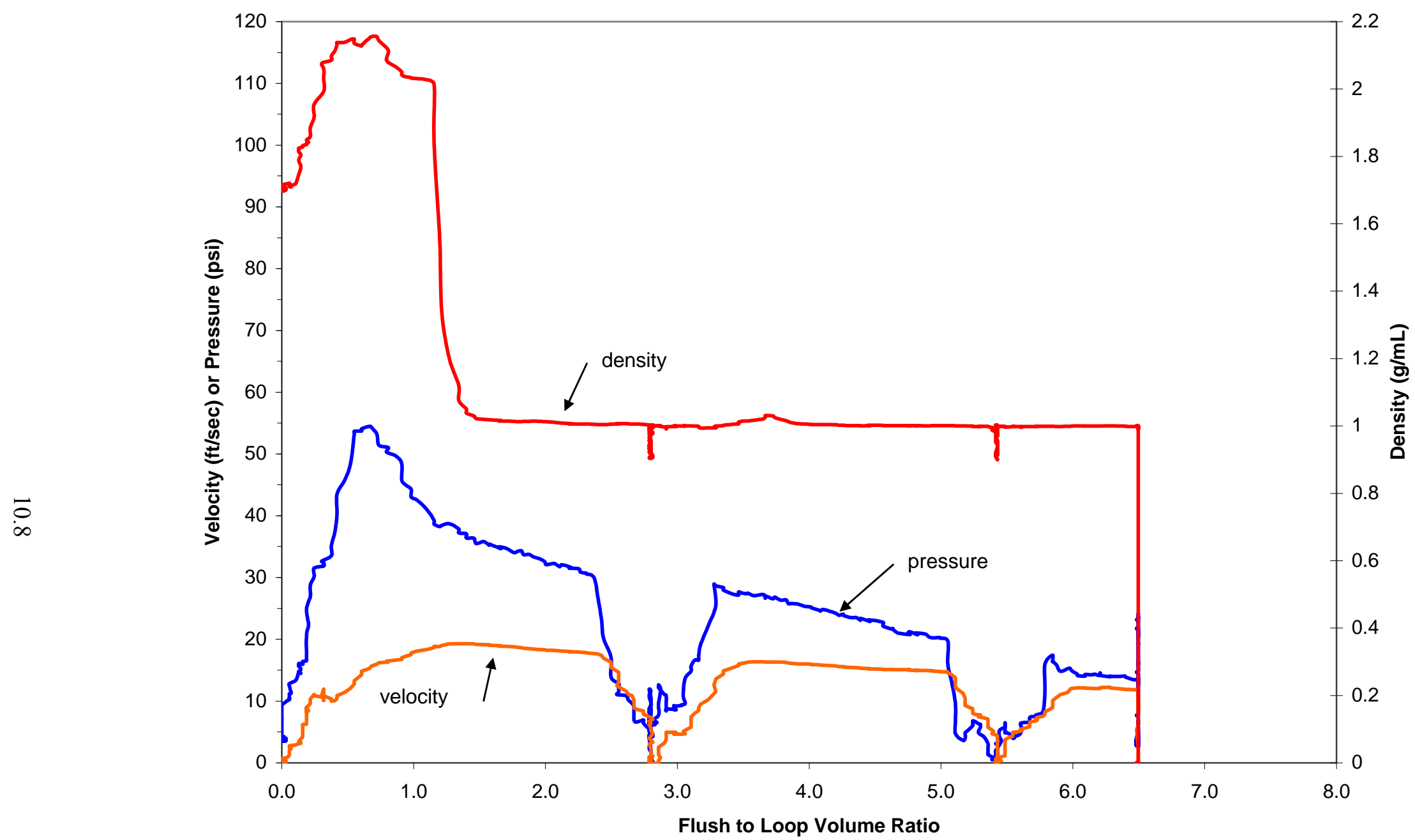

Figure 10.7. Flush Data for HLM Simulant (10- $\mu \mathrm{m}$ stainless steel in thin clay) as a Flush-to-Pipe Loop Volume Ratio Showing Three Complete Flushes 


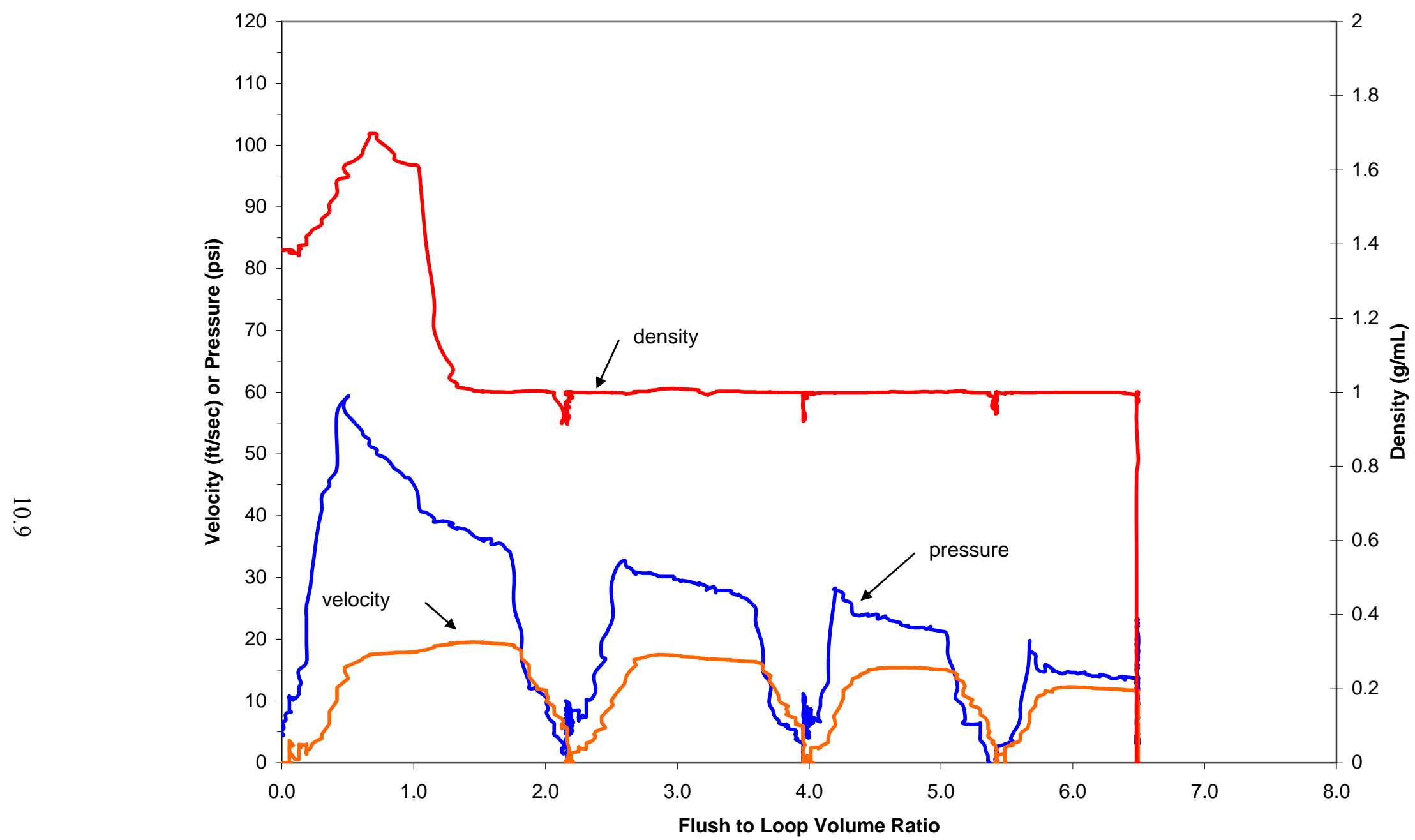

Figure 10.8. Flush Data for MMM Simulant (50- $\mu \mathrm{m}$ alumina in thin clay) as a Flush-to-Pipe Loop Volume Ratio Showing Four Complete Flushes 


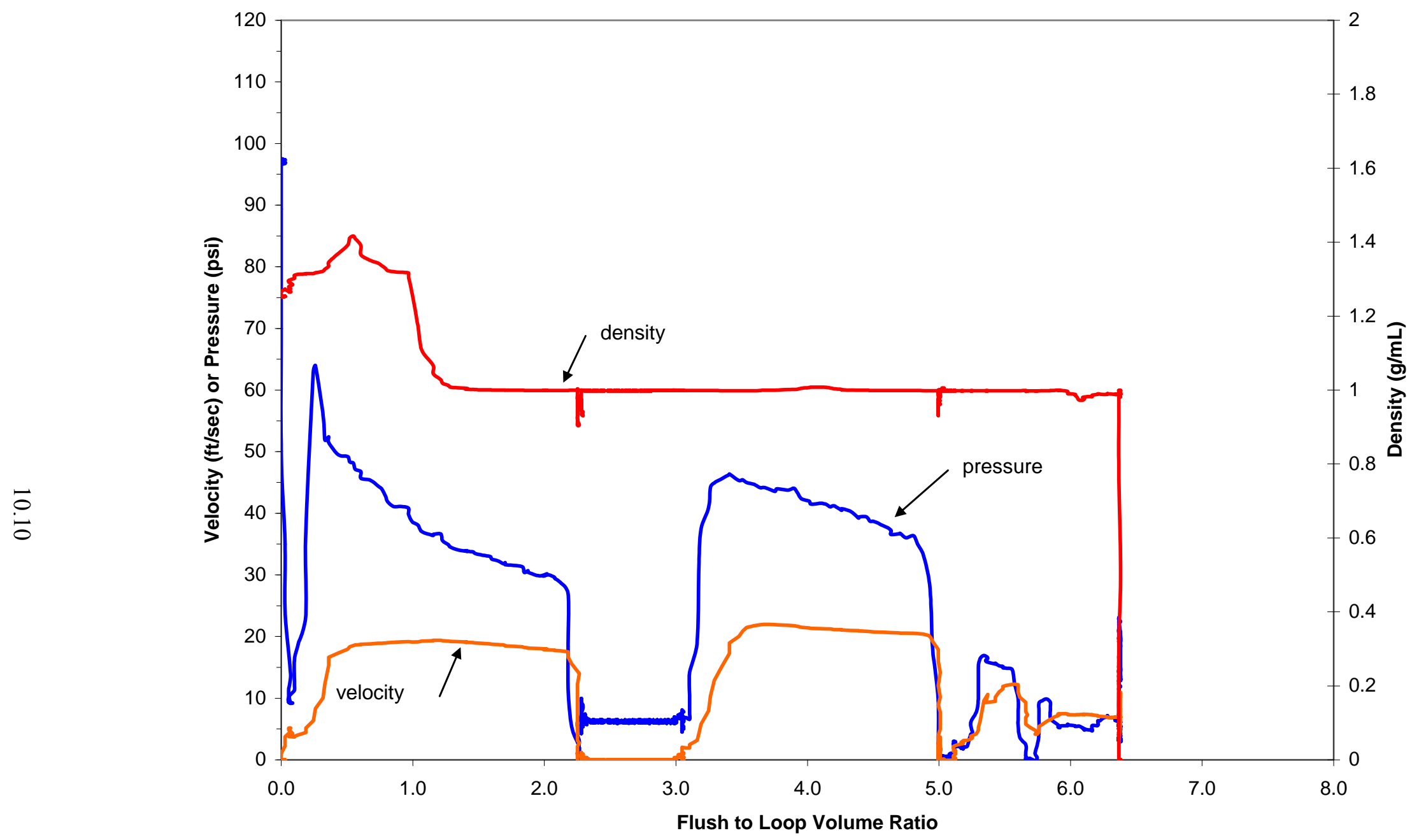

Figure 10.9. Flush Data for LHM Simulant (100- $\mu \mathrm{m}$ glass beads in thin clay) as a Flush-to-Pipe Loop Volume Ratio Showing Three Complete Flushes 


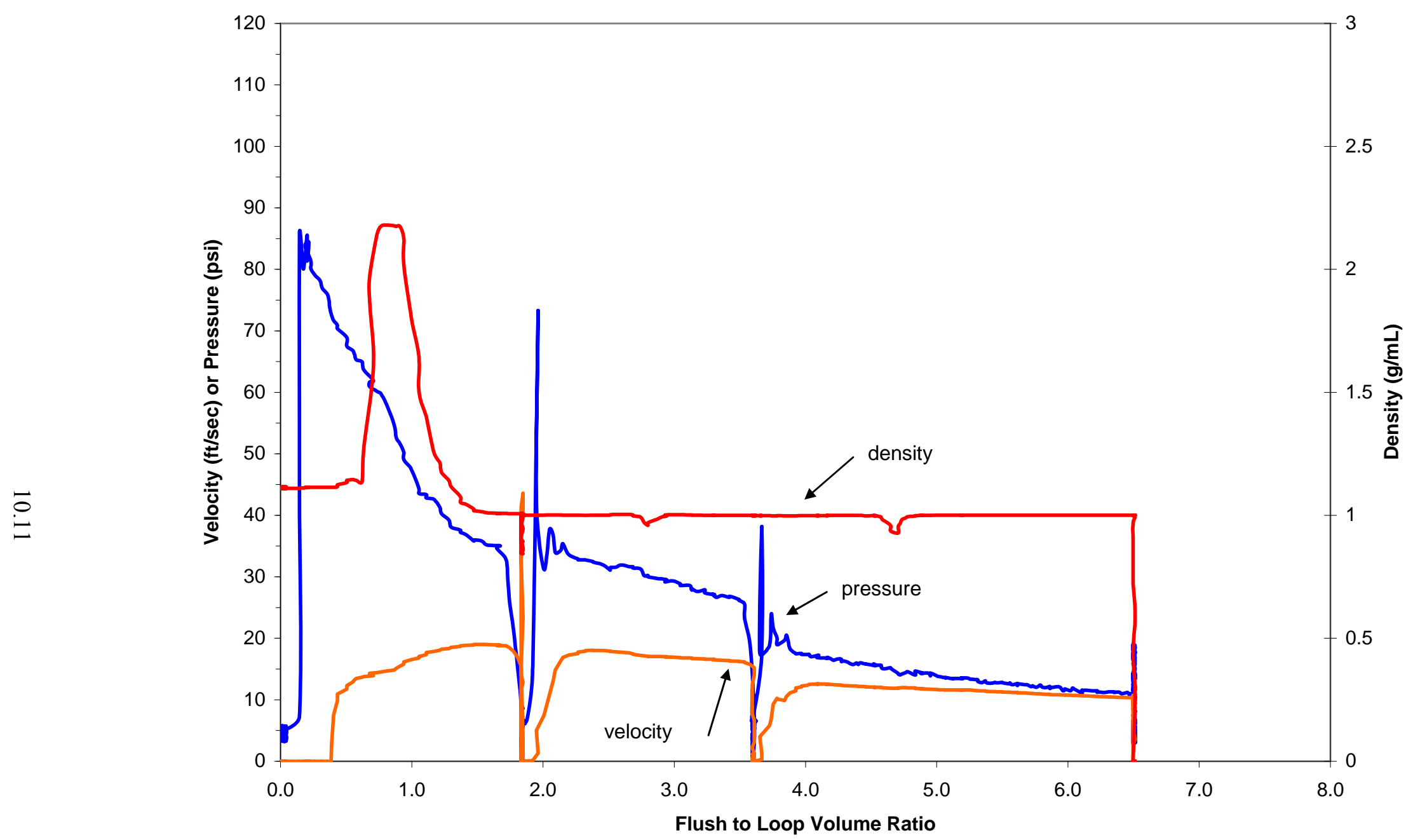

Figure 10.10. Flush Data for HHM Simulant (100- $\mu \mathrm{m}$ stainless steel in thin clay) as a Flush-to-Pipe Loop Volume Ratio Showing Three Complete Flushes 


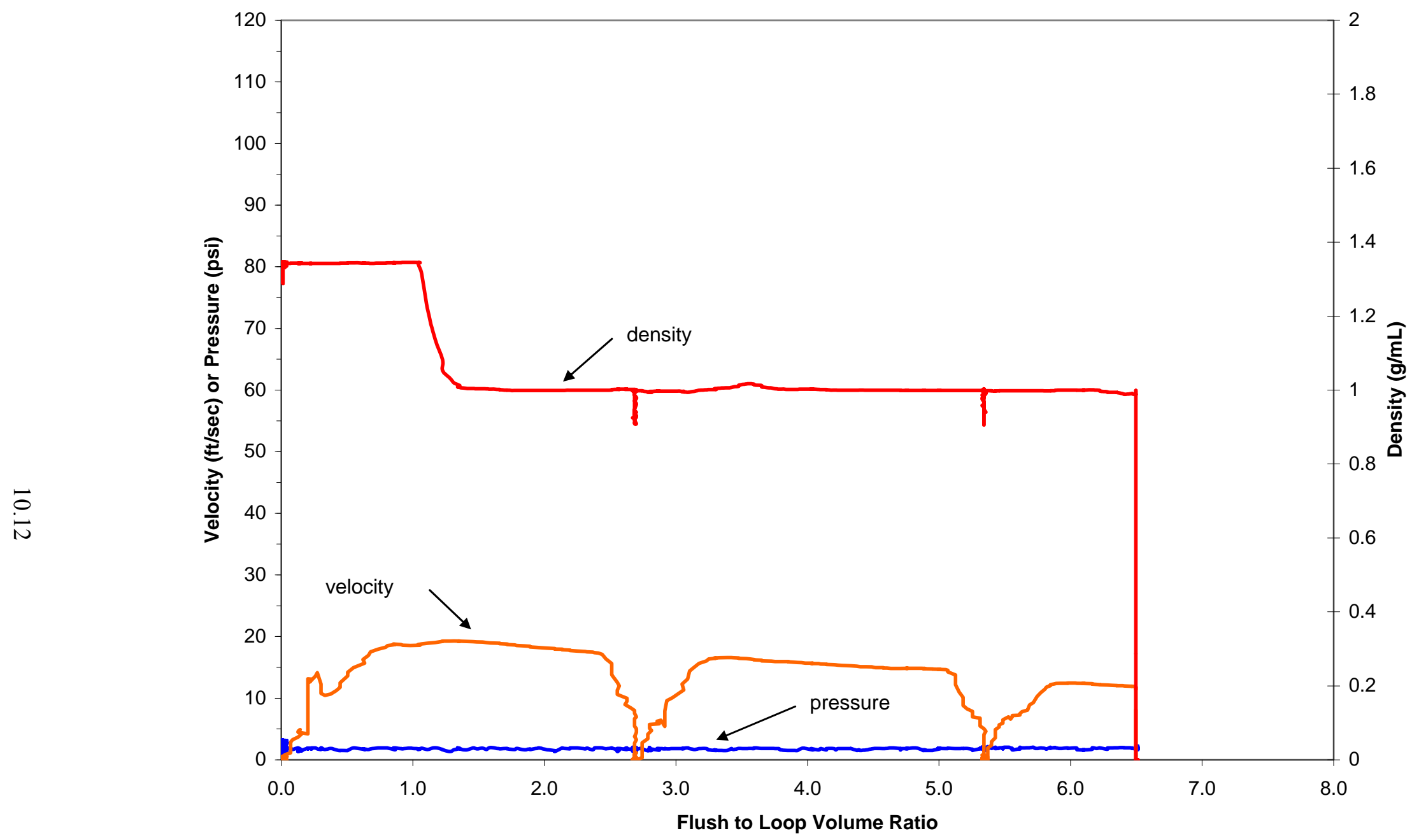

Figure 10.11. Flush Data for LLH Simulant (10- $\mu \mathrm{m}$ glass beads in thick clay) as a Flush-to-Pipe Loop Volume Ratio Showing Three Complete Flushes 


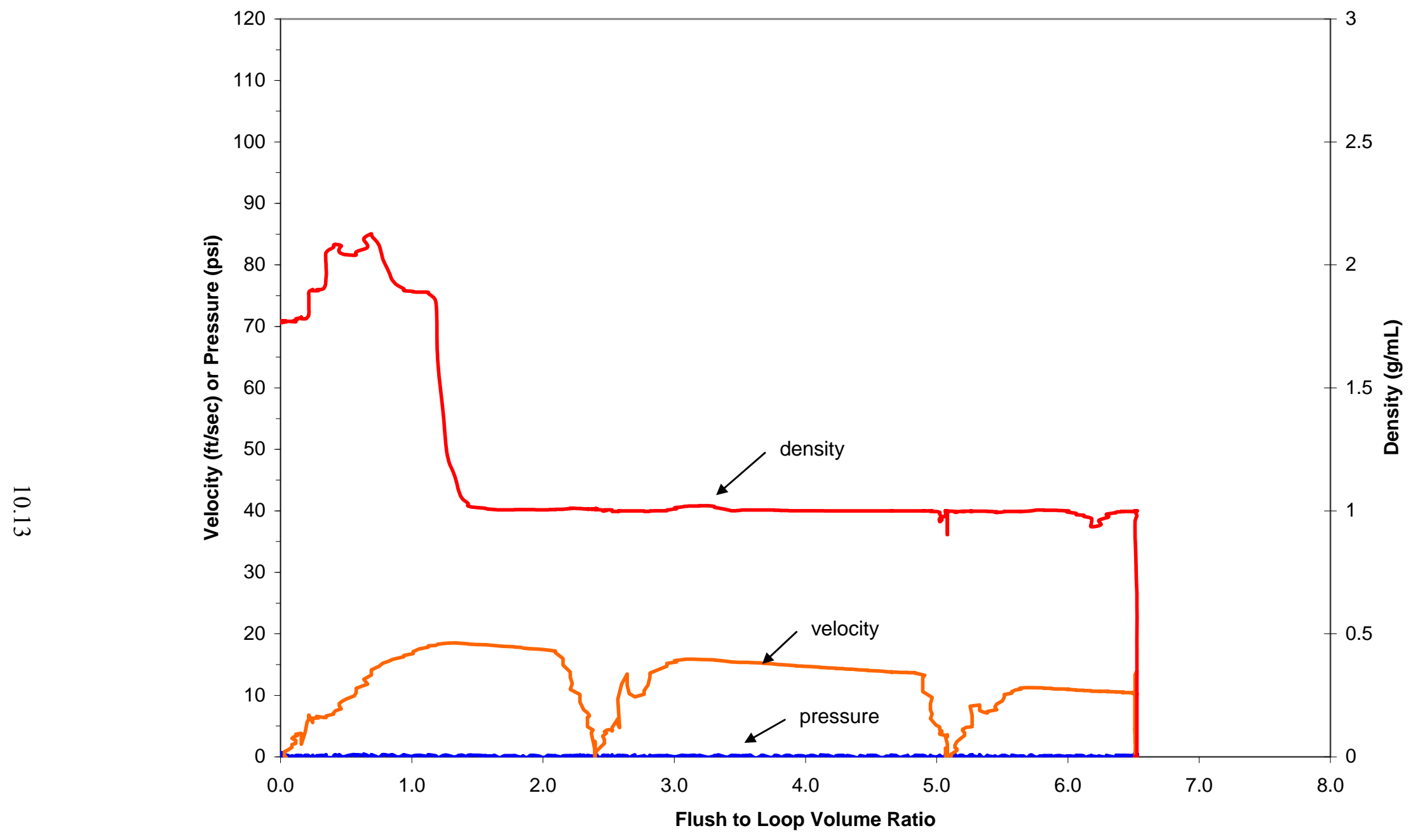

Figure 10.12. Flush Data for HLH Simulant (10- $\mu \mathrm{m}$ stainless steel in thick clay) as a Flush-to-Pipe Loop Volume Ratio Showing Three Complete Flushes. Note that the pressure port valves were closed during flush. 


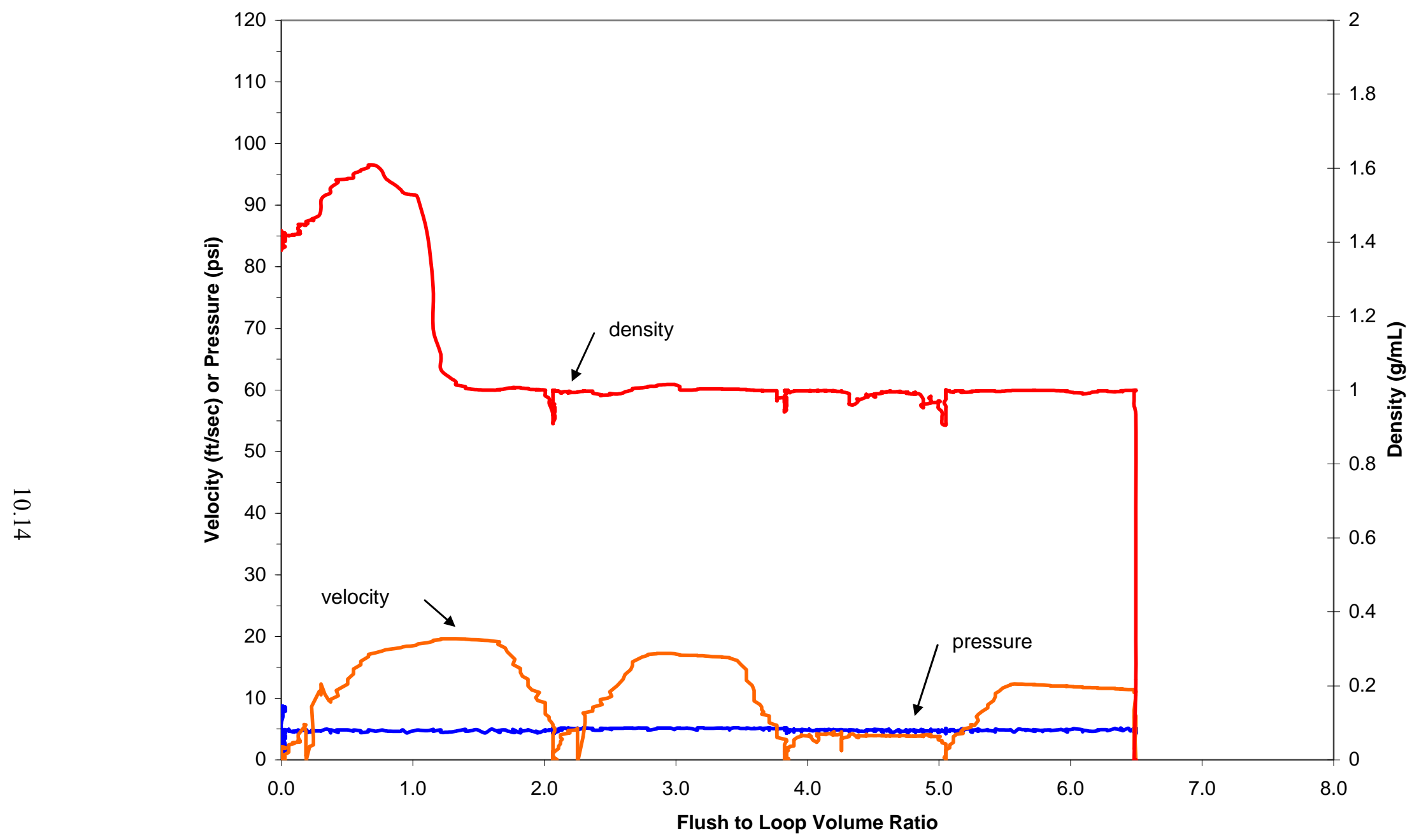

Figure 10.13. Flush Data for MMH Simulant (50- $\mu \mathrm{m}$ alumina in thick clay) as a Flush-to-Pipe Loop Volume Ratio Showing Three Complete Flushes. Note that the pressure port valves were closed during flush. 


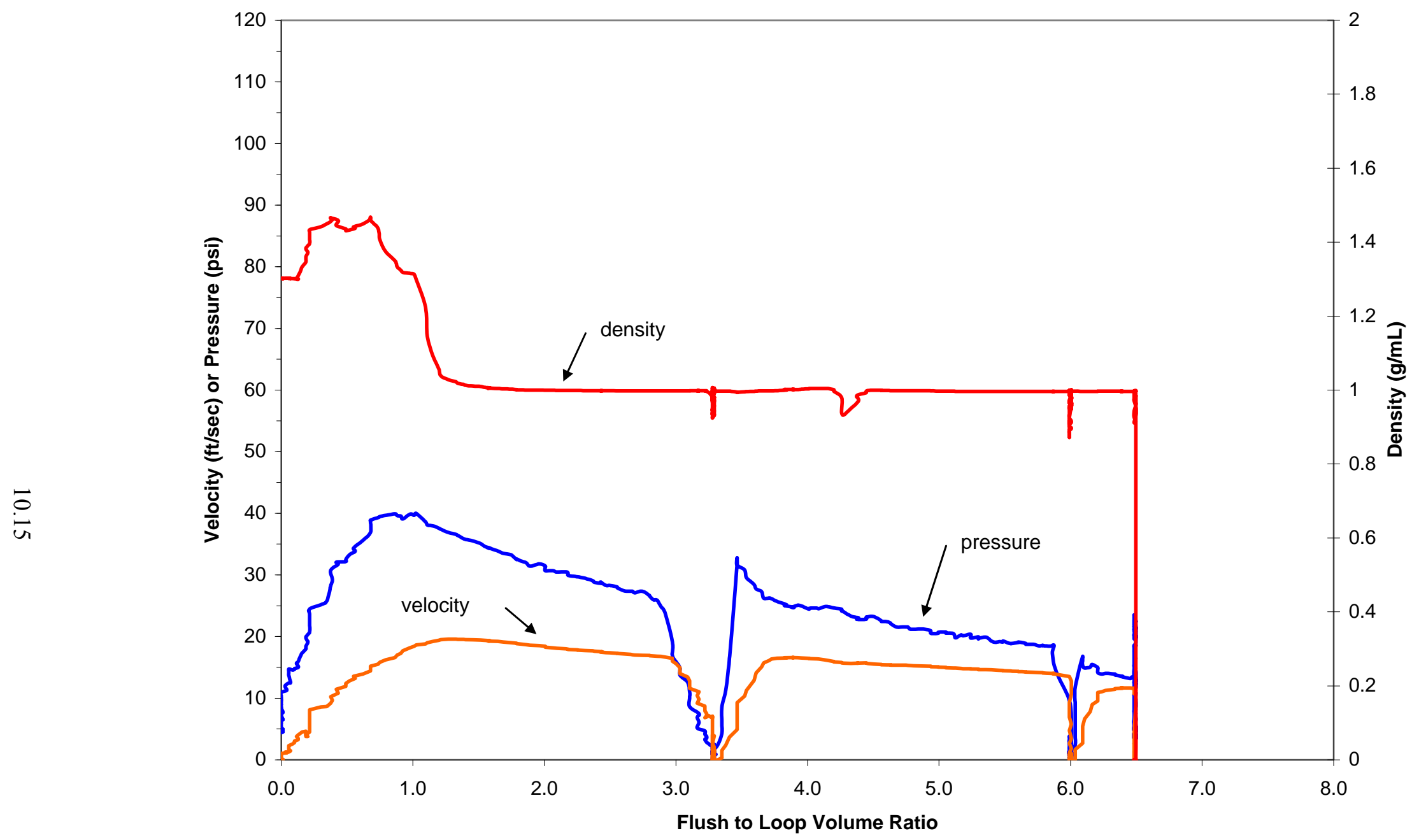

Figure 10.14. Flush Data for LHH Simulant (100- $\mu$ m glass beads in thick clay) as a Flush-to-Pipe Loop Volume Ratio Showing Three Complete Flushes 


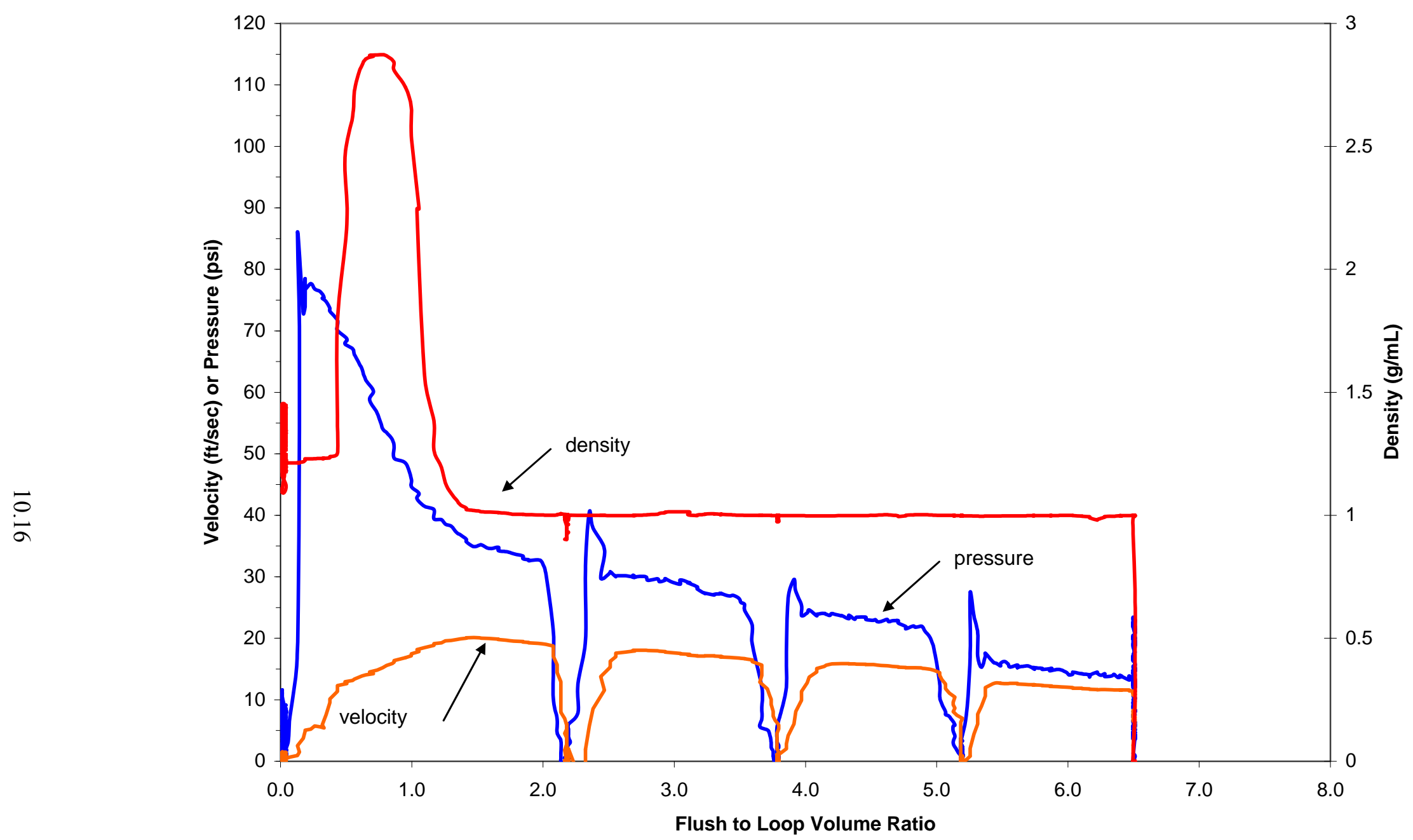

Figure 10.15. Flush Data for HHH Simulant (100 $\mu \mathrm{m}$-stainless steel in thick clay) as a Flush-to-Pipe Loop Volume Ratio Showing Four Complete Flushes 


\subsection{Flush-System Discussion}

The flush system was determined to be effective at removing sediment beds from the piping with the following caveats:

- The pneumatic flush system must be opened slowly to erode the sediment bed from the top down. If the pneumatic flush system is opened quickly, the sediment bed is simply pushed to the nearest corner, and a granular plug develops and completely fills the line cross-sectional area. This was observed in the LHL flush test shown in Figure 10.4. The granular plug was slowly forced through the system by cycling pressure on and off to the line. In the transparent section, the velocity of this granular plug was on the order of inches per second. It took several minutes to remove this granular bed. This behavior was also observed in the HHL and LHM tests where the measured pressure approached 100 psig as shown in Figure 10.5 and Figure 10.9, respectively. It was discovered that cycling the valves open and closed during these situations appeared to accelerate recovery operations. Operation in this mode resulted in water hammer as the flow stopped and started. The water hammer was the source of the data scatter on these plots. Interestingly, the most difficulties occurred with the low-rheology tests. The medium and high rheology likely offers a higher apparent viscosity on the particles in the sediment bed. Consequently, the increase in shear stress due to the higher rheological properties likely promotes plug removal.

- A minimum flush-to-line volume ratio of 1.5 to 1.7 appears to be slightly low. Even though the density of the flush solution exiting the flow loop approaches the flush water density in this volume ration range, a small amount of particles was still observed to be present in the transparent section (approximately covering the bottom $5 \%$ of the pipe section). A minimum flush-to-line volume ratio of 2 would likely produce only a trace amount of particles in the piping.

- The design-guide (Hall 2006) requirements on flush volume and flush velocity were difficult to achieve manually. Achieving these requirements likely would involve a closed-loop feedback system consisting of a flow meter, a level indicator, and an automatic control valve. 



\subsection{Conclusions}

The following conclusions can be made with respect to the test results discussed in this report:

- Small, dense particles settle as superficial velocity decreases toward transition velocity.

- The WTP design-guide protocol of using a 200-mesh (74- $\mu \mathrm{m})$ cut-off for homogeneous carrier fluid in calculations has been shown not to be conservative. Particles below $74 \mu \mathrm{m}$ can settle and cause pipeline plugging under WTP process conditions.

- The WTP design-guide protocol of using a Bingham yield-stress cut-off for homogeneous carrier fluid in calculations has been shown not to be conservative. Particles normally suspended by yield stress in a non-moving system can settle and cause pipeline plugging under pipeline flow conditions.

- Density affects critical velocity far more than particle size.

- The WTP design-guide protocol of using $d_{95}$ particle size in calculations is conservative.

- The design guide specifies the use of the $d_{95}$ density; this term is ambiguous and needs clarification in the design guide. Nonetheless, this value is interpreted to mean the density of the $d_{95}$ particle. This density value is irrelevant for critical velocity calculations. Often this value is unknown, and Equation 1 of the 24590-WTP-GPG-M-0058, Rev 0 design guide (Hall 2006) will be used for design purposes. This equation calculates an average or composite density of all solids in the slurry. The WTP design-guide protocol of using average solids density in the calculations is not conservative. Discrete particles with densities much greater than the average solids density can settle and cause pipeline plugging under WTP process conditions.

- Slurry rheological properties can change dramatically without changing undissolved solids loading.

- The WTP design-guide protocol of using the RPP-9805 correlation (Jewett et al. 2002) that relates solids loading to calculate apparent viscosity of the Hanford Tank Farm feed has been shown to be inaccurate.

- Many physiochemical variables affect slurry rheology, including $\mathrm{pH}$, dissolved salt content, particle size, solid-phase morphology, and system surface chemistry.

- The slurry properties and rheology of each process stream need to be considered rather than primarily the as-received Hanford waste feed stream.

- A little rheology helps in transport; too much hurts.

- As rheological properties increase,

$\circ$ the velocity needed to transport the particles in turbulent flow decreases

0 the transition velocity for turbulent-to-laminar flow also increases, eddies are dampened and particles begin to settle at higher flow rates

- the shear stress on deposited particles increases, allowing them to be transported effectively in laminar flow.

- Four major slurry-transport flow regimes were identified:

- Stable Turbulent Regime 
- Turbulent eddy drag forces are sufficient to suspend the particle buoyant mass.

- Unstable Turbulent Regime

- Turbulent eddy drag forces are not sufficient to suspend the particle buoyant mass.

- Unstable Laminar Regime

○ Turbulent eddy forces are dissipated by viscous forces.

- Pipelines must operate at velocities well above the laminar-to-turbulent transition velocities for the slurries being transported. Near the transition region, the turbulent eddies needed for slurry transport are dampened. This can allow particles to settle and cause pipeline plugging under WTP process conditions.

- Stable Laminar Regime

○ Yield-stress forces dominate the unsheared core region of the pipe flow.

- Yield stress supports particles during transfer.

- Wall shear stress pushes particles deposited along pipe wall.

- The boundary for this flow regime has not been fully defined experimentally.

- The flush system modeled after the WTP design (as of August 2007) appears adequate with a couple of caveats.

- The pneumatic flush system must be opened slowly to erode the sediment bed from the top down.

- If the pneumatic flush system is opened quickly, the sediment bed is simply pushed to the nearest corner, and a granular plug develops and completely fills the line cross-sectional area.

- A minimum flush to a line volume ratio of two would likely produce only a trace amount of particles in the piping. A flush-to-line volume ratio of three would likely produce a line nearly free of solids.

- The design guide on flush volume and flush velocity were difficult to achieve manually. Depending on the process line geometry, flows in the range of 500 to 1,000 gallons per minute can be achieved with this system. Since the piping volumes may be on the order of 50 to 100 gallons, manually closing a valve to hit this target volume may be challenging. Compounding this problem, valves need to be closed slowly to avoid water hammer.

- It is recommended that the flush system be supplemented by a closed-loop feedback system consisting of a flow meter, a level indicator, and automatic control valve for flushing operations. 


\subsection{References}

10 CFR 830. “Nuclear Safety Management.” Code of Federal Regulations, U.S. Department of Energy.

Abulnaga BE. 2002. Slurry Systems Handbook. McGraw-Hill. Online version available at: http://www.knovel.com/knovel2/Toc.jsp?BookID=607\&VerticalID=0. Accessed 05/19/08.

Bae KS, H Lee CG Park, and CS Lee. 1991. "Empirical Correlation for the Minimum Transport Velocity of Multidisperse Slurries in Horizontal Pipes.” Korean Journal of Chemical Engineering 8(2):120-124.

Bechtel National, Inc. (BNI). 2007. Evaluation of Waste Slurry Critical Velocity and Line Flush Capabilities in WTP Process Piping. 24590-WTP-TSP-RT-07-005, Rev. 0, BNI, Richland, Washington.

Bechtold DB, L Jensen, JB Duncan, WS Callaway III, GA Cooke, DL Herting, JC Person, JR Jewett, and JM Tingey. 2002. Particle Property Analyses of High-Level Waste Tank Sludges. HNF-8862 Rev. 0, Fluor Hanford, Inc., Richland, Washington.

N.P. Brown, N.I. Heywood (Editors). 1991. Slurry Handling: Design of solid-liquid systems. Springer.

Chang C, DG Nguyen, and HP Ronningsen. 1999. "Isothermal Start-Up of Pipeline Transporting Waxy Crude Oil.” Journal of Non-Newtonian Fluid Mechanics 87(2-3):127-154.

Churchill SW. 1977. "Friction Factor Spans All Fluid-Flow Regimes.” Chemical Engineering 84(24):91-92.

Cooke R. 2002. "Laminar flow settling: the potential for unexpected problems." BHRG 15th International Conference on Slurry Handling and Pipeline Transport, Hydrotransport 15; Banff, 121-133.

Darby R, R Mun, and DV Boger. 1992. "Prediction of Friction Loss in Slurry Pipes." Chemical Engineering. 99(9):116-119.

Darby, R. 2000. "Pressure Drop for Non-Newtonian Slurries: A Wider Path," Chemical Engineering, May 2000, pp. 64-67.

Davies JT. 1972. Turbulence Phenomena. Academic Press, New York.

Davies JT. 1987. "Calculation of Critical Velocities to Maintain Solids in Suspension in Horizontal Pipes.” Chemical Engineering Science 42(7):1667-1670.

Desouky SE, and MN Al-Awad. 1998. "A New Laminar-to-Turbulent Transition Criterion for YieldPseudoplastic Fluids.” Journal of Petroleum Science and Engineering 19(3-4):171-176.

DOE Order 414.1C. 2005. “Quality Assurance.” U.S. Department of Energy, Washington, D.C.

Dodge DW and AB Metzner. 1959. "Turbulent Flow of Non-Newtonian Systems." AIChE Journal 5:189-204. 
Ferraris C, F de Larrard, and N Martys. 2001. "Fresh Concrete Rheology-Recent Developments.” In: Materials Science of Concrete VI, S Mindess and J Skalny, Editors, American Ceramic Society, Westerville, Ohio.

Gaskill JR, Larson DE, Abrigo GP, Daume JT, Graves RE. 1996. Hanford Waste Vitrification Plant fullscale feed preparation testing with water and process simulant slurries, PNNL-11011, Pacific Northwest National Laboratory, Richland, Washington.

Gillies RG, and CA Shook. 1991. "A Deposition Velocity Correlation for Water Slurries.” Canadian Journal of Chemical Engineering 69(5):1225-1228.

Gillies RG, J Schaan, RJ Sumner, MJ McKibben, and CA Shook. 2000. "Deposition Velocities for Newtonian Slurries in Turbulent Flow.” Canadian Journal of Chemical Engineering 78(4):704-708.

Gillies RG, R Sun, RS Sanders, and J Schaan. 2007. "Lowered Expectations: The Impact of Yield Stress on Sand Transport in Laminar, Non-Newtonian Flows." Journal of the South African Institute of Mining and Metallurgy 107(6):351-357.

Göğüş M, and M Kökpınar. 1993. "Determination of Critical Flow Velocity in Slurry Transporting Pipeline Systems." In: Procedures of the $12^{\text {th }}$ International Conference on Slurry Handling and Pipeline Transport, Hydrotransport 12". pp.743-757.

Göğüş M, and MA Kökpınar. 1999. "An Empirical Expression for the Determination of Critical Flow Velocity in Slurry Transporting Pipeline Systems." In: Proceedings of the $14^{\text {th }}$ International Conference Slurry Handling and Pipeline Transport: Hydrotransport 14. Bury St. Edmunds: Professional Engineering Publishers.

Govier GW, and K Aziz. 1972. The Flow of Complex Mixtures in Pipes. Van Nostrand Reinhold Company, New York.

Guo J, C Tiu, PH Uhlherr, and TN Fang. 2003. "Yielding Behaviour of Organically Treated Anatase $\mathrm{TiO}_{2}$ Suspension.” Korea-Australia Rheology Journal 15(1):9-17.

Hall MN. 2006. Minimum Flow Velocity for Slurry Lines. WTP Project Doc. No. 24590-WTP-GPGM-0058, Rev 0, Bechtel National, Inc., Richland, Washington.

Hanks RW. 1978. "Low Reynolds Number Turbulent Pipeline Flow of Pseudohomogeneous Slurries." Proceedings of the Hydrotransport. 5. BHRA Fluid Engineering, Cranfield, England.

Hanks RW. 1981. "Laminar-Turbulent Transition in Pipeflow of Casson Model Fluids." Journal of Energy Resources Technology-Transactions of the ASME 103:(4)318-321.

Hanks RW, and BH Dadia. 1971. "Theoretical Analysis of the Turbulent Flow of Non-Newtonian Slurries in Pipes." AIChE Journal 17(3):554-557.

Jewett JR, DA Reynolds, SD Estey, L Jensen, NW Kirch, and Y Onishi. 2002. Values of Particle Size, Particle Density, and Slurry Viscosity to Use in Waste Feed Delivery Transfer System Analysis. RPP9805, Rev. 1, Numatech Hanford Corporation, CH2M HILL Hanford Group, Inc., Richland, Washington. 
Kim HT, KS Han, CK Park, and CS Lee. 1986. "Minimum Velocity for Transport of a Sand-Water Slurry through a Pipeline.” International Chemical Engineering 26(4):731-737.

Larson RG. 1998. The Structure and Rheology of Complex Fluids. Oxford University Press, New York.

Laxton PB, and JC Berg. 2006. "Relating clay yield stress to colloidal parameters." Journal of Colloid Interface Science 296(2):749-755.

Liddell KC, and DF Burnett. 2000. Critical Transport Velocity: A Review of Correlations and Models. RPP-7185, Rev. 0, CH2M HILL Hanford Group, Richland, Washington.

Munson BR, DF Young, and TH Okiishi. 1990. Fundamentals of Fluid Mechanics. John Wiley \& Sons, New York.

Oroskar AR, and RM Turian. 1980. "The Critical Velocity in Pipeline Flow of Slurries.” AIChE Journal 26(4):550-558.

Parzonka W, JM Kenchington, and ME Charles. 1981. "Hydrotransport of Solids in Horizontal Pipes: Effects of Solids Concentration and Particle Size on the Deposit Velocity." Canadian Journal of Chemical Engineering 59(3):291-296.

Poloski AP, PA Meyer, LK Jagoda, and PR Hrma. 2003. Technical Basis for LAW Vitrification Stream Physical and Rheological Property Bounding Conditions. PNWD-3398, WTP-RPT-098, Rev. 0, Battelle-Pacific Northwest Division, Richland, Washington.

Poloski AP, ST Arm, OP Bredt, TB Calloway, Y Onishi, RA Peterson, GL Smith, and HD Smith. 2006. Final Report: Technical Basis for HLW Vitrification Stream Physical and Rheological Property Bounding Conditions. PNWD-3675, WTP-RPT-112, Rev. 0, Battelle-Pacific Northwest Division, Richland, Washington.

Poloski AP, BE Wells, JM Tingey, LA Mahoney, MN Hall, SL Thomson, GL Smith, ME Johnson, JE Meacham, MA Knight, MJ Thien, JJ Davis, and Y Onishi. 2007. Estimate of Hanford Waste Rheology and Settling Behavior. PNNL-16857, WTP-RPT-154, Rev. 0, Pacific Northwest National Laboratory, Richland, Washington.

Shah SN, and DL Lord. 1991. "Critical Velocity Correlations for Slurry Transport with Non-Newtonian Fluids.” AIChE Journal 37:6, 863-870.

Shook CA, RG Gillies, and RS Sanders. 2002. Pipeline Hydrotransport with Applications in the OilSand Industry. SRC Publication No. 11508-1E02, Saskatchewan Research Council, Saskatoon, Canada.

Smith GL, and K Prindiville. 2002. Guidelines for Performing Chemical, Physical, and Rheological Properties Measurements. 24590-WTP-GPG-RTD-001 Rev 0, Bechtel National, Inc., Richland, WA.

Steffe JF. 1996. Rheological Methods in Food Process Engineering, $2^{\text {nd }}$ Edition. Freeman Press.

Thomas DG. 1962. "Transport Characteristics of Suspension: Part II, Minimum Transport Velocity for Flocculated Suspension in Horizontal Pipes." AIChE Journal 7:423-430. 
Thomas DG. 1965. "Transport Characteristics of Suspension: VIII. A Note on the Viscosity of Newtonian Suspensions of Uniform Spherical Particles." Journal of Colloid and Interface Science 20(3):267-277.

Thomas AD. 1979. "Predicting the Deposit Velocity for Horizontal Turbulent Pipe Flow of Slurries." International Journal of Multiphase Flow 5(2):113-129.

Thomas AD, Pullum L, and Wilson KC. 2004. "Stabilised laminar slurry flow: review, trends and prognosis." Proc. 16th Int. Conf. Hydraulic Transport of Solids: Hydrotransport 16; Santiago, Chile, BHRG, 701-716.

Toda M, J Yonehara, T Kimura, and S Maeda. 1979. "Transition Velocities in Horizontal Solid-Liquid Two-Phase Flow.” International Chemical Engineering 19(1):145-152.

Turian RM, FL Hsu, and TW Ma. 1987. "Estimation of the Critical Velocity in Pipeline Flow of Slurries.” Powder Technology 51(1):35-47.

Wasp EJ, JP Kenny, and RL Gandhi. 1977. Solid-Liquid Flow Slurry Pipeline Transportation. Tram Tech Publications, Clausthal, Germany.

Wells BE, MA Knight, EC Buck, RC Daniel, SK Cooley, LA Mahoney, PA Meyer, AP Poloski, JM Tingey, WS Callaway III, GA Cooke, ME Johnson, MG Thien, DJ Washenfelder, JJ Davis, MN Hall, G Smith, SL Thomson, and Y Onishi. 2007. Estimate of Hanford Waste Insoluble Solid Particle Size and Density Distribution. PNWD-3824, WTP-RPT-153, Rev. 0, Battelle-Pacific Northwest Division, Richland, Washington.

Wilson KC, Horsley RR, Kealy T, Reizes JA, and Horsley M. 2003 "Direct prediction of fall velocities in non-Newtonian materials." Int. J. Miner. Process. 71:17-30.

Wilson KC and Horsley RR. 2004. "Calculating fall velocities in non-Newtonian (and Newtonian) fluids: a new view." Proc. 16th Int. Conf. Hydraulic Transport of Solids: Hydrotransport 16, Santiago, Chile, BHR Group, Cranfield, UK, pp. 37-46.

Zhou Z, PJ Scales, and DV Boger. 2001. "Chemical and Physical Control of the Rheology of Concentrated Metal Oxide Suspensions.” Chemical Engineering Science 56(9):2901-2920. 


\section{Appendix A}

\section{Background Information and Correlations for Slurry Transport Calculations}





\section{Appendix A}

\section{Background Information and Correlations for Slurry Transport Calculations}

\section{A.1 Rheology Primer}

Rheology is the study of the flow and deformation of materials. When a force (i.e., stress) is placed on an object, the object deforms or strains. Many relationships have been found relating stress to strain for various fluids. The flow behavior of a fluid can generally be explained by considering a fluid placed between two plates of thickness $\mathrm{x}$ (Figure A.1). The lower plate is held stationary while a force, F, is applied to the upper plate of area, A, that results in the plating moving at velocity, $\mathrm{v}$. If the plate moves a length, $\Delta L$, the strain, $\gamma$, on the fluid can be defined by Equation (A.1).

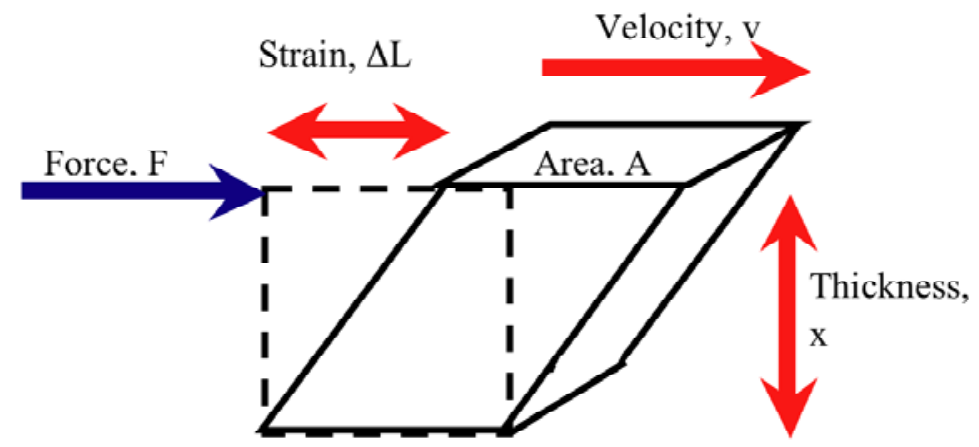

Figure A.1. Diagram of Fluid Flow Between Stationary and Moving Plates

$$
\gamma=\frac{\Delta L}{\mathrm{x}}
$$

The rate of change of strain (also called shear rate), $\dot{\gamma}$, can be defined by Equation (A.2). Because the shear rate is defined as the ratio of a velocity to a length, the units of the variable are the inverse of time, typically s ${ }^{-1}$.

$$
\dot{\gamma}=\frac{d \gamma}{d t}=\frac{d}{d t}\left(\frac{\Delta L}{\mathrm{x}}\right)=\frac{\mathrm{v}}{\mathrm{x}}
$$

Typical shear rates of food-processing applications can be seen in Table A.1. Depending on the application, shear rates in the range of $10^{-6}$ to $10^{7} \mathrm{~s}^{-1}$ are possible. Human perception of a fluid is typically based on a shear rate of approximately $60 \mathrm{~s}^{-1}$. 
The shear stress applied to the fluid can be found by Equation (A.3). Because the shear stress is defined as the ratio of a force to an area, the units of the variable are pressures, typically expressed in $\mathrm{Pa}$ $\left(\mathrm{N} / \mathrm{m}^{2}\right)$.

$$
\tau=\frac{\mathrm{F}}{\mathrm{A}}
$$

Table A.1. Typical Shear Rates in Food-Processing Applications (source: Steffe 1996)

\begin{tabular}{lll}
\hline \multicolumn{1}{c}{ Situation } & Shear Rate Range (1/s) & \multicolumn{1}{c}{ Typical Applications } \\
\hline $\begin{array}{l}\text { Sedimentation of particles in a } \\
\text { suspending liquid }\end{array}$ & $10^{-6}$ to $10^{-3}$ & Medicines, paints, spices in salad dressing \\
$\begin{array}{l}\text { Leveling due to surface tension } \\
\text { Draining under gravity }\end{array}$ & $10^{-2}$ to $10^{-1}$ & Frosting, Paints, printing inks \\
Extrusion & $10^{-1}$ to $10^{1}$ & Vats, small food containers \\
Calendering & $10^{0}$ to $10^{3}$ & $\begin{array}{l}\text { Snack and pet foods, toothpaste, cereals, pasta, } \\
\text { polymers }\end{array}$ \\
Pouring from a bottle & $10^{1}$ to $10^{2}$ & Dough sheeting \\
Chewing and swallowing & $10^{1}$ to $10^{2}$ & Foods, cosmetics, toiletries \\
Dip coating & $10^{1}$ to $10^{2}$ & Foods \\
Mixing and stirring & $10^{1}$ to $10^{2}$ & Paints, confectionery \\
Pipe flow & $10^{1}$ to $10^{3}$ & Food processing \\
Rubbing & $10^{0}$ to $10^{3}$ & Food processing, blood flow \\
Brushing & $10^{2}$ to $10^{4}$ & Topical application of creams and lotions \\
Spraying & $10^{3}$ to $10^{4}$ & Brush painting, lipstick, nail polish \\
High-speed coating & $10^{3}$ to $10^{5}$ & Spray drying, spray painting, fuel atomization \\
Lubrication & $10^{4}$ to $10^{6}$ & Paper \\
\hline & $10^{3}$ to $10^{7}$ & Bearings, gasoline engines \\
\hline
\end{tabular}

The apparent viscosity of the fluid is defined as the ratio of the shear stress to shear rate (see Equation A.4). Often the shear stress and viscosity vary as a function of shear rate. Since the viscosity is defined as the ratio of shear stress to shear rate, the units of the variable are Pa.s. Typically, viscosity is reported in units of centipoise (cP; where $1 \mathrm{cP}=1 \mathrm{mPa} \cdot \mathrm{s})$.

$$
\eta(\dot{\gamma})=\frac{\tau(\dot{\gamma})}{\dot{\gamma}}
$$

For Newtonian fluids, the apparent viscosity is independent of shear rate (Equation A.5). Examples of the viscosity of common Newtonian materials can be seen in Table A.2.

$$
\tau=\eta \dot{\gamma}
$$

where $\tau$ is the shear stress, $\eta$ is the Newtonian viscosity, and $\dot{\gamma}$ is the shear rate. 
Fluids that do not behave as Newtonian fluids are referred to as non-Newtonian fluids. Rheograms or plots of shear stress versus shear rate are typically used to characterize non-Newtonian fluids. Examples of typical rheograms can be seen in Figure A.2.

Table A.2. Viscosities of Several Common Newtonian Fluids (source: Steffe, 1996)

\begin{tabular}{cc}
\hline Material & Viscosity at $20^{\circ} \mathrm{C}(\mathrm{cP})$ \\
\hline Acetone & 0.32 \\
Water & 1.0 \\
Ethanol & 1.2 \\
Mercury & 1.6 \\
Ethylene Glycol & 20 \\
Corn Oil & 71 \\
Glycerin & 1,500 \\
\hline
\end{tabular}

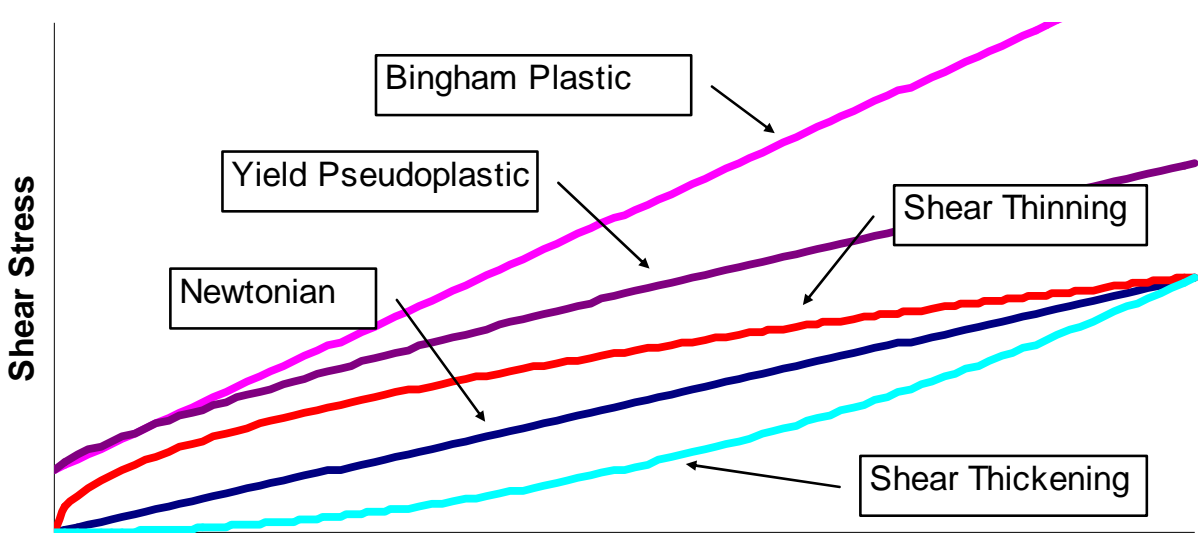

Shear Rate

Figure A.2. Rheograms of Various Fluid Types

Shear-thinning and shear-thickening fluids can be modeled by the Ostwald equation (Equation A.6). If $n<1$, then the material is referred to as pseudoplastic (shear thinning). If $n>1$, then that material is referred to as dilatant (shear thickening). These fluids exhibit decreasing or increasing apparent viscosities as the shear rate increases, depending on whether the fluid is shear thinning or shear thickening, respectively. Since shear-thickening flow behavior is rare, shear-thickening behavior is often an indication of possible secondary flow patterns or other measurement errors.

$$
\tau=m \dot{\gamma}^{n}
$$

where $m$ is the power law consistency coefficient, $n$ is the power law exponent, and $\dot{\gamma}$ is the shear rate.

A rheogram for a Bingham plastic does not pass through the origin. When a rheogram possesses a non-zero y-intercept, the fluid is said to possess a yield stress. A yield stress is a shear-stress threshold that defines the boundary between solid-like behavior and fluid-like behavior. The fluid will not begin to 
flow until the yield-stress threshold is exceeded. For Bingham plastic materials, once enough force has been applied to exceed the yield stress, the material approaches Newtonian behavior at high shear rates (Equation A.7).

$$
\tau=\tau_{B}+\mu_{B} \dot{\gamma}
$$

where $\tau_{B}$ is the Bingham yield stress, $\mu_{B}$ is the plastic viscosity, and $\dot{\gamma}$ is the shear rate.

Fluids that exhibit a non-linear rheogram with a yield stress can be modeled by the three-parameter Herschel-Bulkley equation (Equation A.8). Again, shear-thickening behavior is uncommon, and typically the Hershel-Bulkley power-law exponent is less than unity.

$$
\tau=\tau_{H}+k \dot{\gamma}^{b}
$$

where

$$
\begin{aligned}
\tau_{H} & =\text { yield stress } \\
\mathrm{k} & =\text { Herschel-Bulkley consistency coefficient } \\
\mathrm{b} & =\text { Hershel-Bulkley power law exponent } \\
\dot{\gamma} & =\text { shear rate. }
\end{aligned}
$$

If one desires a rheology model that is non linear but has a yield stress and infinite shear viscosity, the Casson fluid model can be used. This rheology model is shown as Equation (A.9).

$$
\tau^{\frac{1}{2}}=\tau_{C}^{\frac{1}{2}}+\left(\mu_{C} \dot{\gamma}\right)^{\frac{1}{2}}
$$

where $\tau_{C}$ is the yield-stress fit to the Casson fluid model $(\mathrm{Pa})$, and $\mu_{C}$ is the Casson infinite shear viscosity $(\mathrm{Pa} \bullet \mathrm{s})$.

An example of these rheological properties can be considered through a pipeline flow scenario through a 3-inch-ID smooth pipe transporting 90 gallons per minute of fluid. This equates to an average pipeline velocity of $4.1 \mathrm{ft} / \mathrm{sec}$. The fluid is a Bingham plastic with a Bingham yield stress, $\tau_{B}$, of $30 \mathrm{~Pa}$, a Bingham consistency or plastic viscosity, $\mu_{B}$, of $30 \mathrm{cP}$, and a slurry density of $1.2 \mathrm{~kg} / \mathrm{L}$. In this case, the fluid flow will be in the laminar regime with the velocity and apparent viscosity profiles shown in Figure A.3. The flow profile reflects a "plug flow" regime where the center core of the fluid moves at constant velocity. This is because the shear stress in this region does not exceed the yield stress of the fluid and acts as a solid material with an infinite apparent viscosity. At a radius of approximately 1.1 inches, the shear stress in the pipe exceeds the yield stress of the fluid, and the fluid transitions from behaving as a solid to behaving as a "shear thinning" liquid. The apparent viscosity in the sheared region near the pipe wall (1.1- to 1.5-inch radius) drops from an infinite value to approximately $100 \mathrm{cP}$ at the pipe wall. The pressure drop for flow under these conditions is calculated at $9 \mathrm{psig} / 100 \mathrm{ft}$ of straight horizontal pipe. 
The case of a Newtonian fluid with the same pressure drop is then considered. At $90 \mathrm{gpm}$, a Newtonian viscosity of $300 \mathrm{cP}$ is required for a 9-psi/100-ft pressure drop. The flow profiles for this system are shown in Figure A.4. The flow profile shows a parabolic velocity profile that is characteristic of Newtonian, laminar pipe flows. The apparent viscosity in this case is constant at $300 \mathrm{cP}$ throughout the pipe radius.

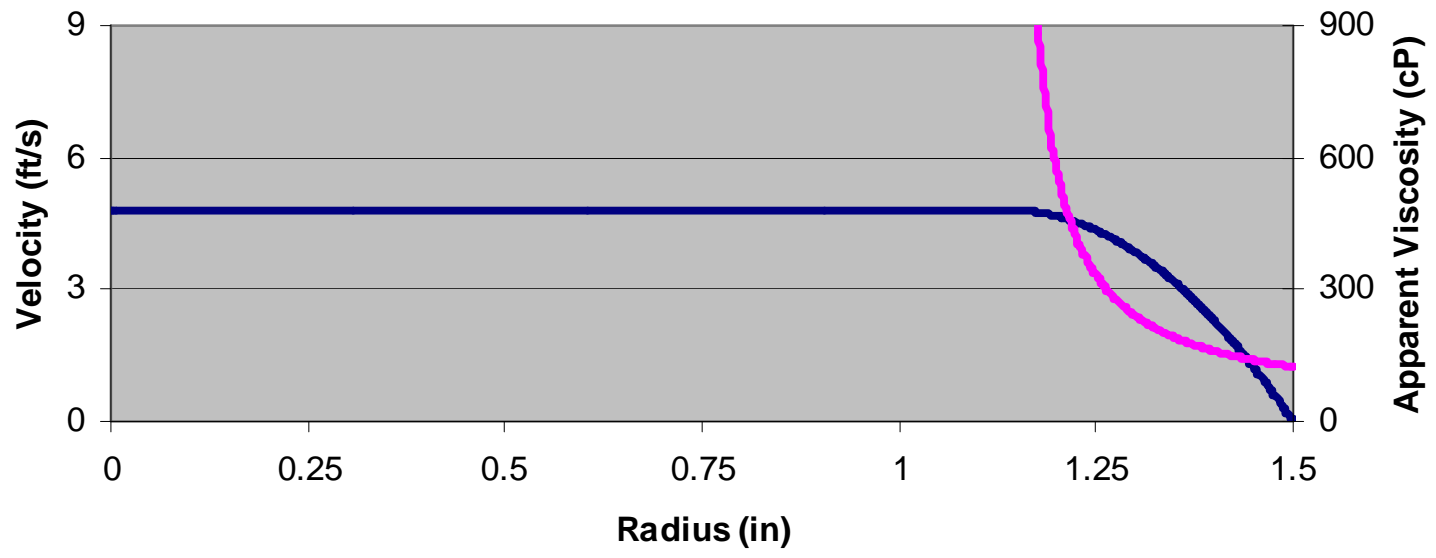

—Velocity —Apparent Viscosity

Figure A.3. Example Flow Profiles for a Bingham Plastic Fluid (30 cP consistency, 30 Pa yield stress) in a 3-inch-ID Smooth Pipe at 90 gpm

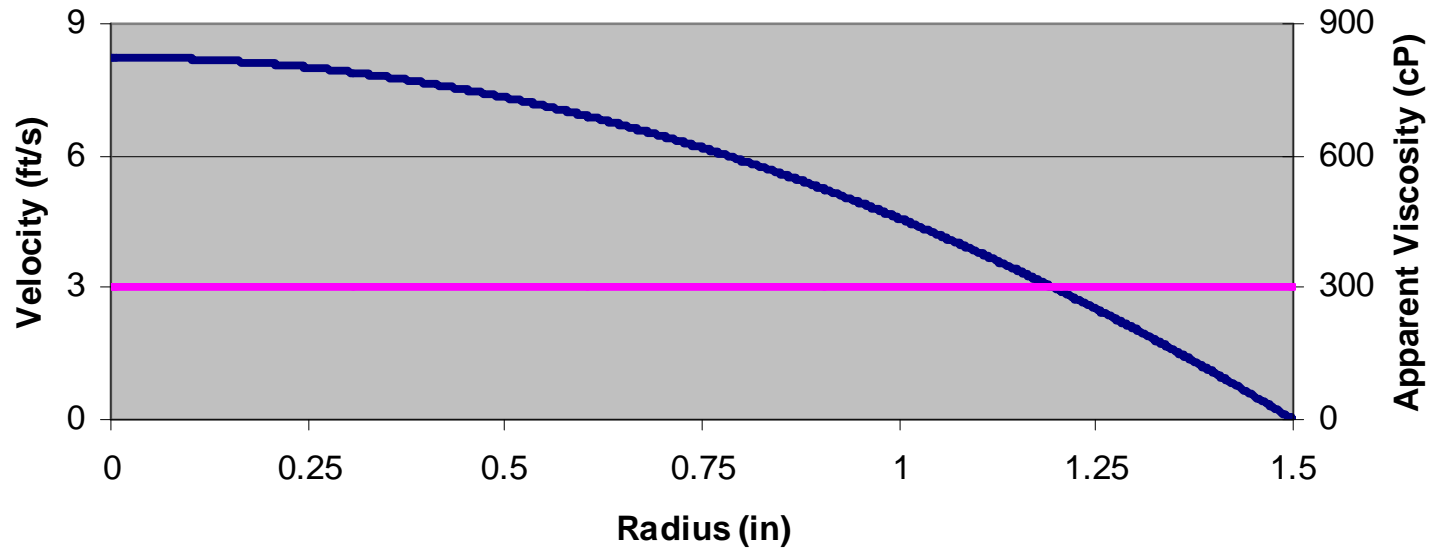

\section{Velocity -Apparent Viscosity}

Figure A.4. Example Flow Profiles for a Newtonian Fluid (30 cP viscosity) in a 3-inch-ID Smooth Pipe at $90 \mathrm{gpm}$ 


\section{A.2 Chemical and Physical Basis for Rheology}

From 2003 to 2006, a DOE Environmental Management Science Project investigated the impacts of colloidal and particle properties on the yield stress and rheology of fluids. The joint University of Washington and PNNL effort produced several papers, including one entitled "Relating clay yield stress to colloidal properties" (Laxton and Berg 2006).

In this paper, Laxton and Berg (2006) explain that Larson (1998, p. 351) has derived a mechanistic relationship for the yield stress of a complex fluid consisting of interacting spherical particles. The approach taken is to model the interactions between the particles as interaction potentials, $\Phi$. At the minima of the interaction potential, the net interparticle force, $F$, is zero. When the system is at equilibrium, the particles will be in a configuration corresponding to one of these minimum interaction potential states. Each equilibrium state has a separation distance between the particles, $D_{0}$, where the interparticle forces are at zero and the interaction potential is at a minimum.

If a shear field is placed on the sample at equilibrium, the separation distance will change, and the forces between the particles will become non-zero. When the shear is removed, these particles will tend back to the equilibrium conditions where the net interparticle forces are zero.

If a greater shear is placed upon the sample, the separation distance will increase, and the net interaction forces between the particles will go through a maximum as the distance increases. At this point, the sample is said to "yield" and begin to flow. Note that this property arises from a purely "cohesive" interaction. For granular systems, these "yield" interactions can be frictional in origin. This discussion is limited to colloidal systems where frictional interactions are negligible.

The bulk yield stress is then proportional to the interparticle forces at that separation distance times the number of particle/particle contacts per unit area being sheared. Laxton and Berg (2006) explain that the number of particle contacts scales with $\phi^{2} / r^{2}$, where $\phi$ is the volume fraction of solids in the sample, and $r$ is the particle radius.

$$
\tau_{y} \propto \frac{\phi^{2}}{r^{2}} F\left(D_{y}\right)
$$

The net interactive force between the particles is equal to the derivative of the interaction potential. Laxton and Berg (2006) state that this is roughly equal to $F\left(D_{y}\right) \approx-\frac{\Phi\left(D_{0}\right)}{D_{0}}$.

$$
\tau_{y} \propto-\frac{\phi^{2}}{r^{2}} \frac{\Phi\left(D_{0}\right)}{D_{0}}
$$

According to Derjaquin, Landau, Verwey, and Overbeek (DLVO) theory, the interaction potential is the sum of van der Waals and electrostatic potentials.

$$
\Phi=\Phi_{v d W}+\Phi_{e}
$$


In the case of monodisperse spherical particles with small relative separation distances, the van der Waals potential can be estimated as shown below:

$$
\Phi_{v d W} \approx \frac{r A_{H}}{12 D}
$$

where $A_{H}$ is the Hamaker constant.

Assuming constant surface charge density on the surface of the particles, the electrostatic potential can be estimated as follows:

$$
\Phi_{e} \approx 2 \pi \varepsilon \varepsilon_{0} r \psi_{s}^{2} \ln \left[\frac{1}{1-\exp (-\kappa D)}\right]
$$

where $\varepsilon=$ dielectric constant of the medium

$\varepsilon_{0}=$ permittivity of a vacuum

$\psi_{s} \quad=$ surface charge potential

$\kappa=$ inverse Debye length.

Combining these equations and replacing the surface potential with the zeta potential, $\zeta$, produces the following relation:

$$
\tau_{\mathrm{y}} \propto \frac{\phi^{2}}{\mathrm{r}}\left\{\frac{\mathrm{A}_{\mathrm{H}}}{12 \mathrm{D}_{0}^{2}}-\frac{2 \pi \varepsilon \varepsilon_{0}}{\mathrm{D}_{0}} \zeta^{2} \ln \left[\frac{1}{1-\exp \left(-\kappa \mathrm{D}_{0}\right)}\right]\right\}
$$

The inverse Debye length can be approximated as

$$
\kappa=\sqrt{\frac{2 N_{a} e^{2} I}{\varepsilon \varepsilon_{0} k T}}
$$

where $N_{a}=$ Avogadro's Number

$e=$ elementary charge

$I=$ ionic strength of the electrolyte

$k=$ Boltzmann's constant

$T=$ temperature. 
As explained by Laxton and Berg (2006), an estimate of the Hamaker constant can be obtained from Lifshitz theory for two identical solid phases (1) across a medium (2) using the following equation:

$$
A_{H}=\frac{3}{4} k T\left(\frac{\varepsilon_{1}-\varepsilon_{2}}{\varepsilon_{1}+\varepsilon_{2}}\right)^{2}+\frac{3 h v_{e}}{16 \sqrt{2}} \frac{\left(n_{1}^{2}-n_{2}^{2}\right)^{2}}{\left(n_{1}^{2}+n_{2}^{2}\right)^{3 / 2}}
$$

where $h$ is the Planck constant, $n$ is the refractive index, and $v_{e}$ is the frequency of electron cloud oscillations (typically 3 to $5 \times 10^{15} \mathrm{~s}^{-1}$ ).

The particle separation at minimum interaction potential typically has a value of 0.1 to $0.3 \mathrm{~nm}$.

Zhou et al. (2001) normalized and simplified the above equation and obtained the following relation.

$$
\frac{\tau_{y}}{\tau_{y \max }}=1-\frac{24 \pi \varepsilon \varepsilon_{0} \zeta^{2} D_{0}^{2}}{A_{H}\left(1+e^{\kappa D_{0}}\right)}
$$

where $A_{H}=$ Hamaker constant of the slurry

$D_{0}=$ minimum separation distance between the particles in a flocculated state

$\varepsilon_{r}=$ dielectric constant

$\varepsilon_{0} \quad=$ permittivity of a vacuum

$\kappa=$ inverse Debye length.

Guo et al. (2003) make the following statement about this equation:

A feature of the above equation is to correlate the normalized shear yield stress with surface chemistry independently of the structural properties of particles. Thus, all normalized data of yield stress for the suspensions with a variety of solids concentrations should collapse onto a single master curve in a plot against the square of zeta potential or $\mathrm{pH}$.

The equations presented above represent ideal situations where the DLVO theory can be directly applied. These types of situations are rare in practice, but the effect of several physical and chemical variables can be predicted. A table summarizing the expected trends is shown below. Note that chemical properties in the form of $\mathrm{pH}$ and dissolved salt content have a dramatic effect on the yield stress of a suspension.

If one takes a stable suspension and begins to alter the $\mathrm{pH}$ or dissolved salt content, the suspension will begin as a stable suspension with a low yield stress. As the zeta potential approaches the iso-electric point (IEP), the suspension will begin to flocculate or form a solid structure that increases yield stress. Further shifts in $\mathrm{pH}$ or salt content will stabilize the suspension as the suspension progresses away from the IEP. However, even further changes in $\mathrm{pH}$ or salt content result in the inverse Debye length being the dominant factor rather than the zeta potential, and the suspension will again flocculate and increase the yield stress. The dynamics of this system are discussed in Table A.2. 
Table A.3. Physicochemical Factors that Affect Slurry Rheology

\begin{tabular}{|c|c|c|c|}
\hline $\begin{array}{l}\text { Physicochemical } \\
\text { Factors }\end{array}$ & $\begin{array}{c}\text { Colloidal Variables } \\
\text { Affected }\end{array}$ & Functionality & Result \\
\hline $\begin{array}{l}\mathrm{pH} \text { and Dissolved } \\
\text { Salt Content }\end{array}$ & Zeta potential & $\begin{array}{l}\text { Moderate } \mathrm{pH} \text { values tend to } \\
\text { push Zeta potential to zero at } \\
\text { IEP. }\end{array}$ & $\begin{array}{c}\text { Measured yield stress } \\
\text { approaches maximum at IEP. }\end{array}$ \\
\hline $\begin{array}{l}\text { Dissolved Salt } \\
\text { Content }\end{array}$ & Inverse Debye length & $\begin{array}{l}\text { Increases with increasing ionic } \\
\text { strength. }\end{array}$ & $\begin{array}{c}\text { Measured yield stress } \\
\text { approaches maximum under } \\
\text { high ionic strength conditions. }\end{array}$ \\
\hline $\begin{array}{l}\text { Undissolved Solids } \\
\text { Content }\end{array}$ & $\begin{array}{l}\text { Solid-particle volume } \\
\text { fraction }\end{array}$ & $\begin{array}{l}\text { Volume fraction increases } \\
\text { with undissolved solids } \\
\text { content. }\end{array}$ & $\begin{array}{l}\text { Maximum yield stress } \\
\text { increases with increasing } \\
\text { undissolved solids content. }\end{array}$ \\
\hline Particle Size & Particle radius & Increases with particle size. & $\begin{array}{l}\text { Maximum yield stress } \\
\text { decreases with increasing } \\
\text { particle size. }\end{array}$ \\
\hline $\begin{array}{l}\text { Solid and liquid } \\
\text { chemical morphology } \\
\text { and speciation }\end{array}$ & $\begin{array}{l}\text { Hamaker constant and } \\
\text { equilibrium particle } \\
\text { separation distance }\end{array}$ & $\begin{array}{l}\text { Varies with chemical particle } \\
\text { morphology and system } \\
\text { chemical composition. }\end{array}$ & $\begin{array}{l}\text { Varies with chemical particle } \\
\text { morphology and system } \\
\text { chemical composition. }\end{array}$ \\
\hline
\end{tabular}

\section{A.3 Rheology of Real Systems}

Ferraris et al. (2001, pp. 215-241) provide an equation that describes how the Bingham plastic consistency parameter varies with solids concentration:

$$
\mu_{B}=m e^{n \phi}
$$

where $\mu_{B}$ is the Bingham consistency in $\mathrm{cP}, \phi$ is the solids volume fraction, and $m$ and $n$ are the fitting parameters.

Zhou et al. (2001) provide an equation that describes how the Bingham yield stress varies with solids concentration (Equation A.20). This equation is of similar form to the shear-strength equation.

$$
\tau_{B}=p \phi^{q}
$$

where $\tau_{B}$ is the Bingham yield stress, $\phi$ is the solids volume fraction, and $p$ and $q$ are the fitting parameters.

The parameters $m, n, p$, and $q$ are a function of solids and liquid composition, $\mathrm{pH}$, temperature, the state/configuration of particle aggregation, and particle size. These equations are typically valid only when the chemistry of the slurry is held constant while varying the solids loading. This can be achieved by diluting the slurry with decanted or separated interstitial liquid that is at equilibrium with the slurry solids. The yield-stress equation based on DLVO theory predicts a dependence of an inverse relationship between particle size and yield stress. However, many experiments have demonstrated that yield stress is 
proportional to the inverse square of particle size. On this topic, Zhou et al. (2001) state "Although the perplexing question of why the additional inverse size dependence observed is open for discussion, it is not likely to come from the force dependence (which is well predicted by traditional DLVO equations)."

The fluid rheology of waste slurries intended for treatment at WTP is dependent on many complex parameters that may change dramatically during physical and chemical processing. For instance, as they exist in the tanks, the initial waste tank solids are suspended/slurried in a saturated salt solution. Retrieval and transport of these slurries to waste pre-treatment operations is facilitated via water dilution. The dilution will lower the $\mathrm{pH}$, the dissolved salt content, and the solids volume fraction due to dissolution of salt crystals, particle size, and solid/liquid chemistry. Extreme batch-to-batch variability due to the different processes that generated the waste in a particular waste tank being retrieved also adds to the complexity.

The retrieved high-level waste (HLW) slurry feed will then be processed through a leaching operation where the solid aluminum and chromium phases will be removed with the remaining solids concentrated to a target of $20-\mathrm{wt} \%$ undissolved solids. The HLW slurry interstitial liquid will be replaced with "inhibited water," which is a 0.01-M sodium hydroxide solution, to form a pretreated HLW sludge stream. Again, changes to $\mathrm{pH}$, dissolved salt content, solids volume fraction, particle size, and solid/liquid chemistry will occur. The permeate from this leaching operation, also called pretreated lowactivity waste (LAW), will change because dissolved aluminum and chromium species will be added as well as water needed for unit operation.

Lastly, glass-former chemicals (GFCs) will be added to the pretreated HLW sludge and LAW streams. The GFC composition will vary from batch to batch and contains a mixture of soluble and insoluble species. Again, changes to $\mathrm{pH}$, dissolved salt content, solids volume fraction, particle size, and solid/liquid chemistry will occur.

\section{A.4 Gillies and Shook Model}

The Gillies and Shook (1991) model has been updated in an article authored by Gillies et al. (2000). In this article, Gillies et al. (2000) proposes that the principal independent variable should be the Archimedes Number. The Archimedes Number can be defined as the ratio of gravitational forces on the particle corrected for buoyancy to viscous forces on the particle. Mathematically, this can be expressed as,

$$
A r=\frac{4}{3} \frac{g d^{3}(S-1) \rho_{f}^{2}}{\mu_{f}^{2}}
$$

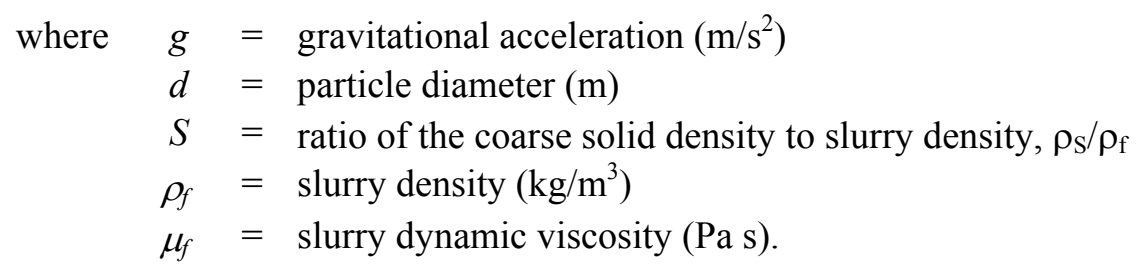


The Froude Number can be defined as the ratio of inertial forces in the fluid flow over the gravitational forces the particle must overcome to stay suspended in the flow. Mathematically, this can be expressed as,

$$
F r=\frac{V_{c}}{\sqrt{g D(S-1)}}
$$

where $V_{c}$ is the deposition velocity $(\mathrm{m} / \mathrm{s})$, and $D$ is the pipe diameter $(\mathrm{m})$.

Shook et al. (2002) provide a relation between the Archimedes Number and the Froude Number.

$$
\begin{aligned}
& 540<A r \quad F r=1.78 A r^{-0.019} \\
& 160<A r<540 \mathrm{Fr}=1.19 A r^{0.045} \\
& 80<A r<160 F r=0.197 A r^{0.4}
\end{aligned}
$$

This relationship allows the critical deposition velocity to be calculated over a range of particle and carrier fluid properties in non-dimensional form. This equation has the benefit of not containing a slurry concentration term given by similar critical-velocity equations. This simplification allows for increased engineering flexibility at the slight expense in correlation accuracy.

Gillies and Shook (1991) also presented a carrier fluid concept where a criterion for a homogeneous carrier "vehicle" is established. This criterion was set at material that passes through a 200 mesh sieve (i.e. $74 \mu \mathrm{m}$ opening). Results from this report show that particles smaller than $74 \mu \mathrm{m}$ can behave as heterogeneous solids and deposit in pipelines. Consequently, the homogeneous fraction criterion is much more complex than previously anticipated. For simplicity, bulk fluid property data were used for density and viscosity inputs in this model rather than calculated carrier fluid values.

\section{A.5 Turbulence Model}

The purpose of this section is to derive the Shook et al. (2002) correlation shown in Equation A.23 from first principles. The first principle approach will allow for alternative forms of the Shook et al. (2002) correlations to be derived based on different physical mechanisms. These alternative correlations may help explain experimental observations associated with sedimentation in non-Newtonian systems. The initial force balance in this experimental approach is based upon the work of Davies (1987). The sedimentation force on a particle due to buoyant gravitational forces is given as:

$$
\text { sedimentation force }=\left(\rho_{S}-\rho_{L}\right) g\left(\frac{\pi d^{3}}{6}\right)
$$


The current approach discussed below differs from Davies (1987) in that the hindered settling concentration terms are neglected, which is consistent with the Shook et al. (2002) correlations. The drag force on a particle generated by eddy-velocity fluctuations, $v^{\prime}$, is given as:

$$
\text { eddy fluctuation force }=\text { eddy pressure } \times \text { area }=\frac{1}{2} C_{D} \rho_{L}\left(v^{\prime}\right)^{2}\left(\frac{\pi d^{2}}{4}\right)
$$

Davies (1987) considers the drag coefficient, $C_{D}$, to be a constant, which is consistent with the Newton's Law regime. In the current approach, the drag coefficient is considered to be a variable of particle size and density; this is consistent with the transitional and Stokes' Law regimes. At the criticalvelocity condition, the sedimentation force will be equal to the eddy fluctuation force.

$$
v^{\prime}=\sqrt{\left(\frac{4}{3}\right) \frac{g d(S-1)}{C_{D}}}
$$

As explained by Davies (1987), the size of the eddies, $l_{E}$, is assumed to be approximately equal to the diameter of the particles being suspended. This is because smaller eddies will not be capable of lifting the particles, while larger eddies cannot closely approach the bottom of the pipe where the solids are sedimented.

$$
l_{E} \approx d
$$

The eddy fluctuation velocity is given in terms of turbulent energy dissipation, $\varepsilon$, from Davies (1972).

$$
v^{\prime} \propto\left(\varepsilon l_{E}\right)^{1 / 3} \rightarrow(\varepsilon d)^{1 / 3}
$$

Davies (1972) also provides an equation relating turbulent energy dissipation to the friction factor and pipeline velocity.

$$
\varepsilon=2 \frac{V^{3}}{D} f
$$

By substituting Equations A.26 and A.29 into Equation A.28, the proportional equation shown below is formed.

$$
V f^{1 / 3} \propto \sqrt{\frac{g D(S-1)}{C_{D}}}\left(\frac{d}{D}\right)^{1 / 6}
$$

Shook et al. (2002) present the drag coefficient as the power law function of the Archimedes number.

$$
C_{D}=a A r^{b}
$$


Both $a$ and $b$ depend on the Archimedes number. Shook et al. (2002) provide constant values for $a$ and $b$ over four regimes that cover the viscous-force dominated or Stokes' Law region to the inertial-force dominated or Newton's Law region. The resulting drag coefficient relationships for these regimes are:

$$
\begin{array}{cc}
A r \leq 24 \text { (Stokes' Law) } & C_{D}=576 A r^{-1} \\
24<A r \leq 2760 & C_{D}=80.9 A r^{-0.475} \\
2760<A r \leq 46100 & C_{D}=8.61 A r^{-0.193} \\
46100<A r \text { (Newton's Law) } & C_{D}=1.09
\end{array}
$$

The equality shown below can be realized by making the drag coefficient substitution in Equation A.30 and grouping all constants together with a term, $s$.

$$
F r=s\left(\frac{d}{D}\right)^{1 / 6} A r^{-b / 2} f^{-1 / 3}
$$

The Blausis formula is used to determine the friction factor for Newtonian fully turbulent flow in smooth pipes (Munson et al. 1990).

$$
f=0.079 \mathrm{Re}^{-1 / 4}
$$

The pipe Reynolds Number is a combination of three dimensionless groups.

$$
\operatorname{Re}=\sqrt{\frac{3}{4}}\left(\frac{d}{D}\right)^{-3 / 2} \sqrt{A r} F r
$$

The following relation can be found by making these substitutions and grouping all constants into a single term, $s_{t u r b}$.

$$
F r=s_{\text {turb }}\left(\frac{d}{D}\right)^{1 / 22} A r^{1 / 22^{-6 b / 11}}
$$

Using the values for the Archimedes Number exponent, $b$, four different relationships can be obtained, depending on the functionality of the Archimedes Number to the drag coefficient.

$$
\begin{array}{lll}
b=-1 \rightarrow & F r & \approx s_{\text {turb }} A r^{0.59} \\
b=-0.475 \rightarrow & F r & \approx s_{\text {turb }} A r^{0.30} \\
b=-0.193 \rightarrow & F r & \approx s_{\text {turb }} A r^{0.15} \\
b=0 \rightarrow & F r & \approx s_{\text {turb }} A r^{0.045}
\end{array}
$$

The ratio of the particle to pipe diameter is taken to the 0.045 exponent, which results in the value being nearly constant. In the Newton's law case with $b$ being zero, the Archimedes Number is taken to the 0.045 power. Interestingly, this relation is similar to the Shook et al. (2002) critical-velocity correlation for $160<A r<540$ (see Equation A.23). For the case of Archimedes number less than 80, the 
surface area of the particles begins to increase and viscous forces generated by drag on the surface of the particles begins to dominate inertial and gravitational forces. This may result in the Shook et al. (2002) correlations taking a form similar to Equation A.26 when $b \neq 0$ when the Archimedes number is less than 80 .

\section{A.6 Bingham Plastic Transition-Velocity Calculations}

A precondition for applying critical-velocity correlations, such as the Oroskar and Turian (1980) and Gillies et al. (2000) correlations, is that the slurry flow is turbulent. The first step in calculating the laminar-to-turbulent transition velocity for Bingham plastic fluids is to determine the Hedstrom Number as shown below (Hanks and Dadia 1971; Desouky and Al-Awad 1998; Chang et al. 1999):

$$
H e=\frac{D^{2} \rho_{f} \tau_{B}}{\mu_{B}^{2}}
$$

where $H e=$ Hedstrom Number (dimensionless)

$D=$ pipe diameter $(\mathrm{m})$

$\rho_{f}=$ fluid or slurry density $\left(\mathrm{kg} / \mathrm{m}^{3}\right)$

$\tau_{B}=$ yield-stress fit to Bingham plastic model $(\mathrm{Pa})$

$\mu_{B}=$ Bingham-plastic infinite-shear viscosity $(\mathrm{Pa} \bullet \mathrm{s})$.

The next step is to solve the following implicit equation for the ratio between the yield stress and wall shear stress, $\xi$ (Hanks and Dadia 1971; Desouky and Al-Awad 1998; Chang et al. 1999):

$$
\frac{\xi}{(1-\xi)^{3}}=\frac{H e}{16,800}
$$

The transition Reynolds Number, $R e_{t}$, can then be calculated. This value represents the transition from laminar-to-turbulent flow. The equation for this calculation is shown below (Hanks and Dadia 1971; Desouky and Al-Awad 1998; Chang et al. 1999):

$$
\operatorname{Re}_{t}=H e \frac{\left[1-\frac{4}{3} \xi+\frac{1}{3} \xi^{4}\right]}{8 \cdot \xi}
$$


Alternatively, Wasp et al. (1977) proposed an equation that can be solved directly (see Equation (A.41)). The coefficient of this equation was modified from 1500 to 1050 in order to produce a laminar-to-turbulent transition Reynolds number of 2100 under Newtonian conditions (i.e. when the Hedstrom number is zero). This modification results in a closer fit to the data generated by Equations (A.39) and (A.40).

$$
\operatorname{Re}_{t}=1050\left(1+\sqrt{1+\frac{H e}{4500}}\right)
$$

The velocity of the fluid in the pipe, $V$, can then be calculated from the definition of the Reynolds Number as shown below:

$$
\operatorname{Re}=\frac{D V \rho_{f}}{\mu_{B}} \rightarrow V_{t}=\frac{\mu_{B}}{D \rho_{f}} \operatorname{Re}_{t}
$$

\section{A.7 Bingham Plastic Pressure-Drop Calculations}

Calculating the pressure drop required to maintain a given flow rate with a Bingham plastic fluid is usually performed with a dimensionless parameter called the Fanning friction factor, $f$ (Hanks and Dadia 1971; Desouky and Al-Awad 1998; Chang et al. 1999).

$$
f=\frac{D \Delta P}{2 L \rho_{f} V^{2}}
$$

where

$$
\begin{aligned}
f & =\text { Fanning friction factor (dimensionless) } \\
D & =\text { pipe diameter }(\mathrm{m}) \\
\Delta P & =\text { Pressure drop }(\mathrm{Pa}) \\
L & =\text { Length of pipe }(\mathrm{m}) \\
\rho_{f} & =\text { fluid or slurry density }\left(\mathrm{kg} / \mathrm{m}^{3}\right) \\
V & =\text { fluid bulk velocity }(\mathrm{m} / \mathrm{s}) .
\end{aligned}
$$

The following correlations for laminar $\left(R e<R e_{t}\right)$ and turbulent $\left(R e>R e_{t}\right)$ flow regimes exist between the Fanning friction factor, Hedstrom Number, and Reynolds Number (Abulnaga 2002; Hanks 1978; Chang et al. 1999):

$$
\begin{aligned}
& f_{\text {lam }}=\frac{16}{\operatorname{Re}}\left[1+\frac{H e}{6 \operatorname{Re}}-\frac{H e^{4}}{3 f_{\text {lam }}^{3} \operatorname{Re}^{7}}\right] \operatorname{Re}<\operatorname{Re}_{t} \\
& f_{\text {turb }}= \begin{cases}10^{c} \operatorname{Re}^{-0.193} & \operatorname{Re}>\operatorname{Re}_{t} \\
c=-1.47\left[1+0.146 \exp \left(-2.9 \times 10^{-5} H e\right)\right] & \end{cases}
\end{aligned}
$$


At Reynolds Numbers near the transition region, Darby et al. (1992) suggests the following p-norm relation for combining the laminar and turbulent contributions:

$$
f_{\text {tot }}=\left(f_{\text {lam }}^{p}+f_{\text {turb }}^{p}\right)^{\frac{1}{p}}
$$

Where the p-norm exponent follows the relation:

$$
p=1.7+\frac{40000}{\operatorname{Re}}
$$

Accounting for the roughness of the pipe, $\varepsilon_{r r}$, requires a Newtonian friction factor to be determined. Churchill (1977) proposed a friction factor correlation for both laminar and turbulent flow regimes. This equation is shown below:

$$
f_{\text {Church }}=2 \cdot\left[\left(\frac{8}{\mathrm{Re}}\right)^{12}+\frac{1}{(A+B)^{1.5}}\right]^{1 / 12}
$$

The parameters A and B are determined from the Reynolds Number and pipe roughness ratio from the equations shown below:

$$
\begin{gathered}
A=\left\{-2.457 \cdot \ln \left[\left(\frac{7}{\mathrm{Re}}\right)^{0.9}+\frac{0.27 \varepsilon_{r r}}{D}\right]\right\}^{16} \\
B=\left(\frac{37530}{\mathrm{Re}}\right)^{16}
\end{gathered}
$$

The Churchill (1977) equation is used to predict the pressure drop over a length of pipe for Newtonian, rough wall, laminar, and turbulent flows. Govier and Aziz (1972) suggest that a simple scaling relationship can be used to account for pipe roughness with non-Newtonian flows; see Equation (A.51):

$$
\frac{f_{\text {rough }}}{f_{\text {smooth }}}=\frac{f_{\text {Church,rough }}}{f_{\text {Church,smooth }}}
$$




\section{A.8 Casson Fluid Transition-Velocity Calculations}

The first step in calculating the laminar-to-turbulent transition velocity for Casson fluids is to determine the Casson Number as shown below (Hanks 1981):

$$
C a=\frac{D^{2} \rho_{f} \tau_{C}}{\mu_{C}{ }^{2}}
$$

where $\mathrm{Ca}$ is the Casson Number (dimensionless), $\tau_{C}$ is the yield-stress fit to the Casson fluid model $(\mathrm{Pa})$, and $\mu_{C}$ is the Casson infinite shear viscosity $(\mathrm{Pa} \bullet \mathrm{s})$.

A similar equation to the Wasp (1977) equation for Bingham plastic transitional flow is proposed below:

$$
\operatorname{Re}_{t}=1050\left[1+\left(1+\frac{\mathrm{Ca}}{370}\right)^{0.4}\right]
$$

A plot of the Hanks (1981) laminar-to-turbulent transition data as a function of Casson number is shown in Figure A.5. The curve representing Equation A.28 is also plotted and shows a satisfactory fit to the Hanks (1981) theoretical data.

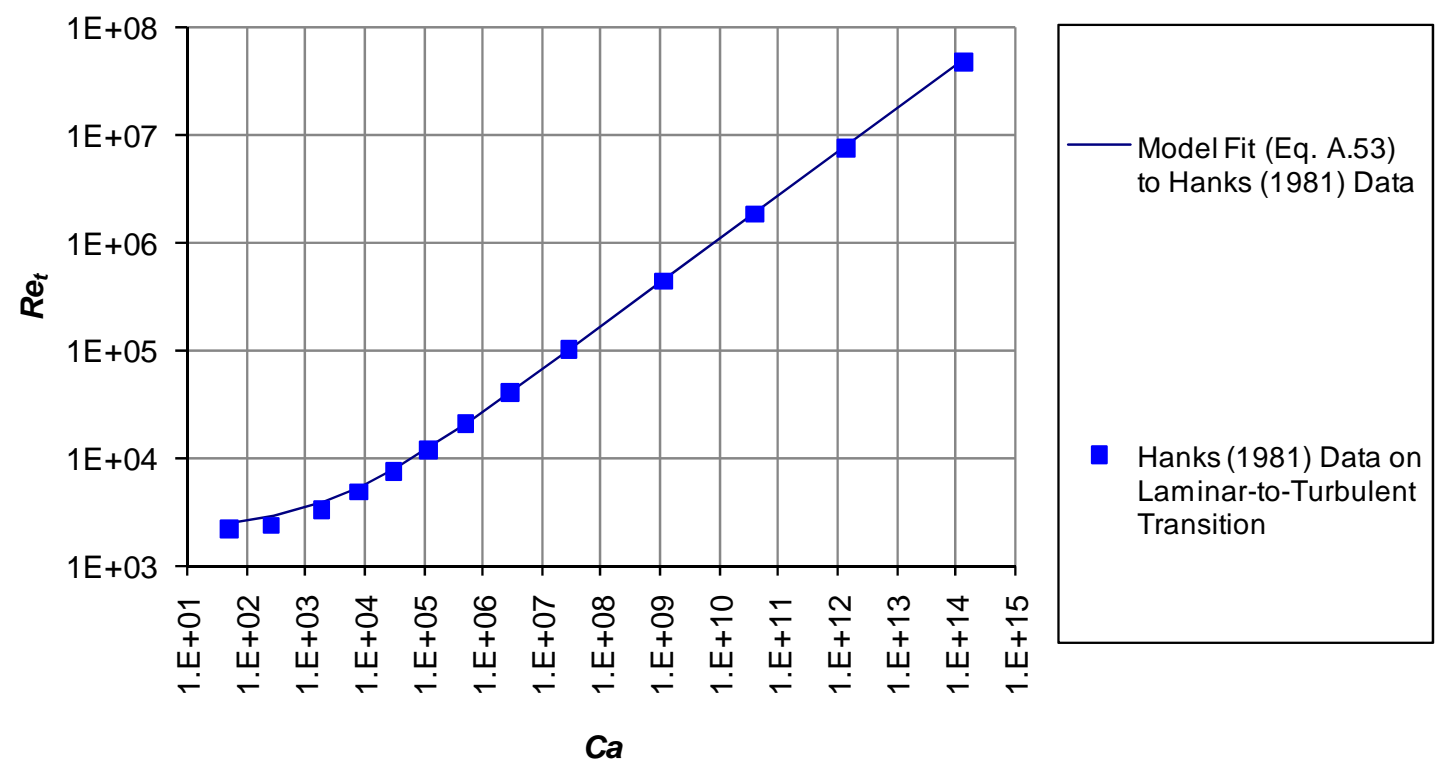

Figure A.5. Laminar-to-Turbulent Transition Reynolds Number as a Function of Casson Number 
The velocity of the fluid in the pipe can then be calculated from the definition of the Reynolds Number as shown below:

$$
\operatorname{Re}=\frac{D V \rho_{f}}{\mu_{B}} \rightarrow V_{t}=\frac{\mu_{C}}{D \rho_{f}} \operatorname{Re}_{t}
$$

\section{A.9 Casson Fluid Pressure-Drop Calculations}

Calculating the pressure drop required to maintain a given flow rate with a Casson fluid involves solving the Wilson-Thomas turbulent flow equation. This equation presented by Shook et al. (2002) is shown by Equation (A.55).

$$
V=V_{N}+2.5 u^{*} \ln \left(\frac{1-\xi}{1+\frac{2}{3} \xi^{\frac{1}{2}}+\frac{1}{3} \xi}\right)+u^{*}\left[\xi(2.5+1.25 \xi)+11.6\left(\frac{2}{3} \xi^{\frac{1}{2}}+\frac{1}{3} \xi\right)\right]
$$

where $V_{N}$ is the equivalent Newtonian fluid velocity $(\mathrm{m} / \mathrm{s}), u^{*}$ is the $\sqrt{\frac{\tau_{w}}{\rho_{f}}}$, the friction velocity $(\mathrm{m} / \mathrm{s})$, and $\xi$ is the ratio of the fluid yield stress to wall shear stress, $\tau_{\mathrm{C}} / \tau_{\mathrm{w}}$.

The velocity $V_{N}$ is evaluated using a friction factor determined at the Reynolds Number,

$$
\operatorname{Re}=\frac{D V_{N} \rho_{f}}{\mu_{C}}\left(1-\xi^{\frac{1}{2}}\right)^{2}
$$

Shook et al. (2002) also present the equation for laminar Casson fluid flow as shown below.

$$
\frac{8 V}{D}=\left(\frac{\tau_{w}}{\mu_{C}}\right)\left[1-\frac{16}{7} \sqrt{\xi}+\frac{4}{3} \xi-\frac{1}{21} \xi^{4}\right]
$$


Appendix B

\section{Lasentec Results}





\section{Appendix B}

\section{Lasentec Results}

\section{B.1 Lasentec Results for LLL (top) LLM (middle) LLH (bottom) Systems; For Information Only}

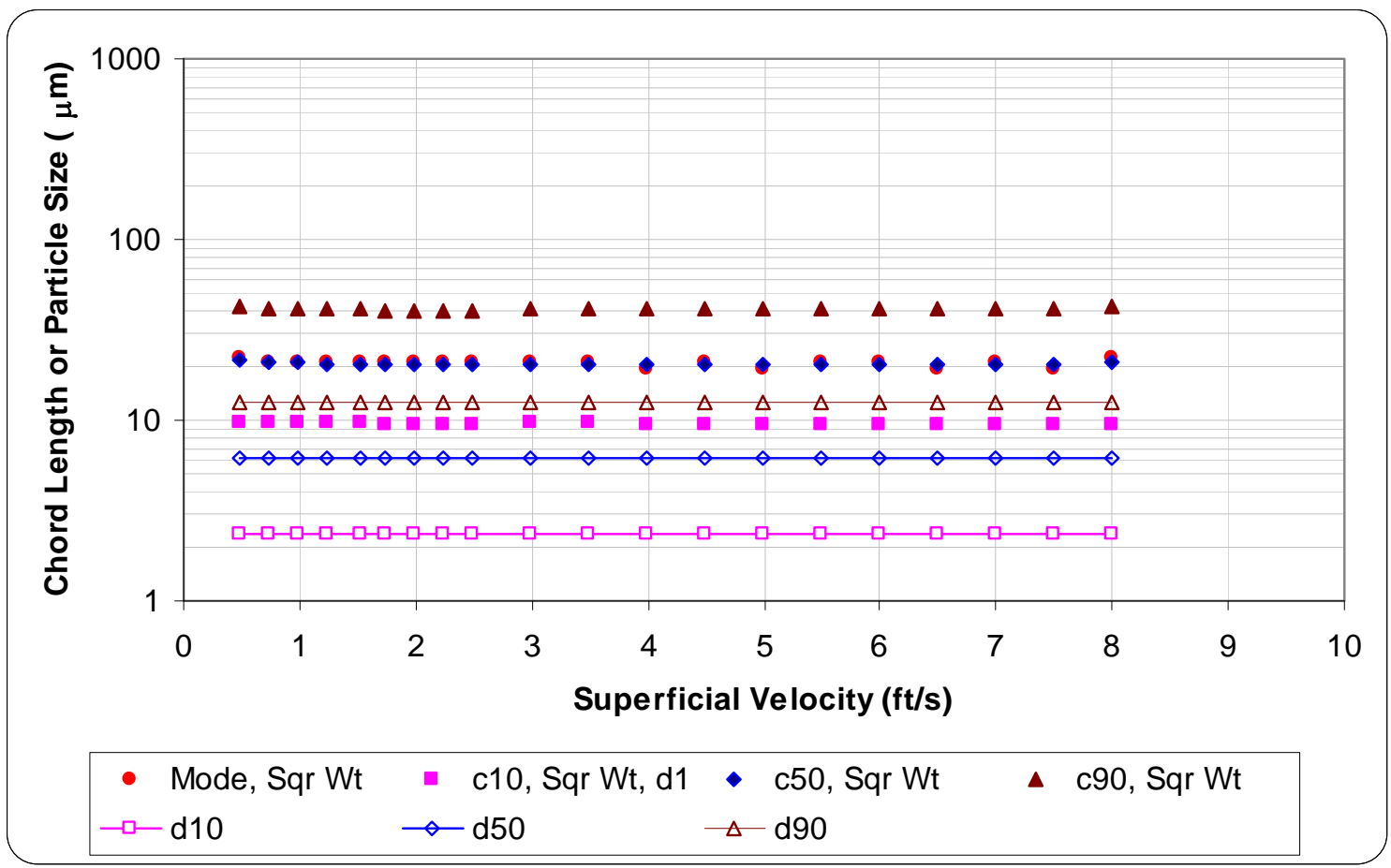



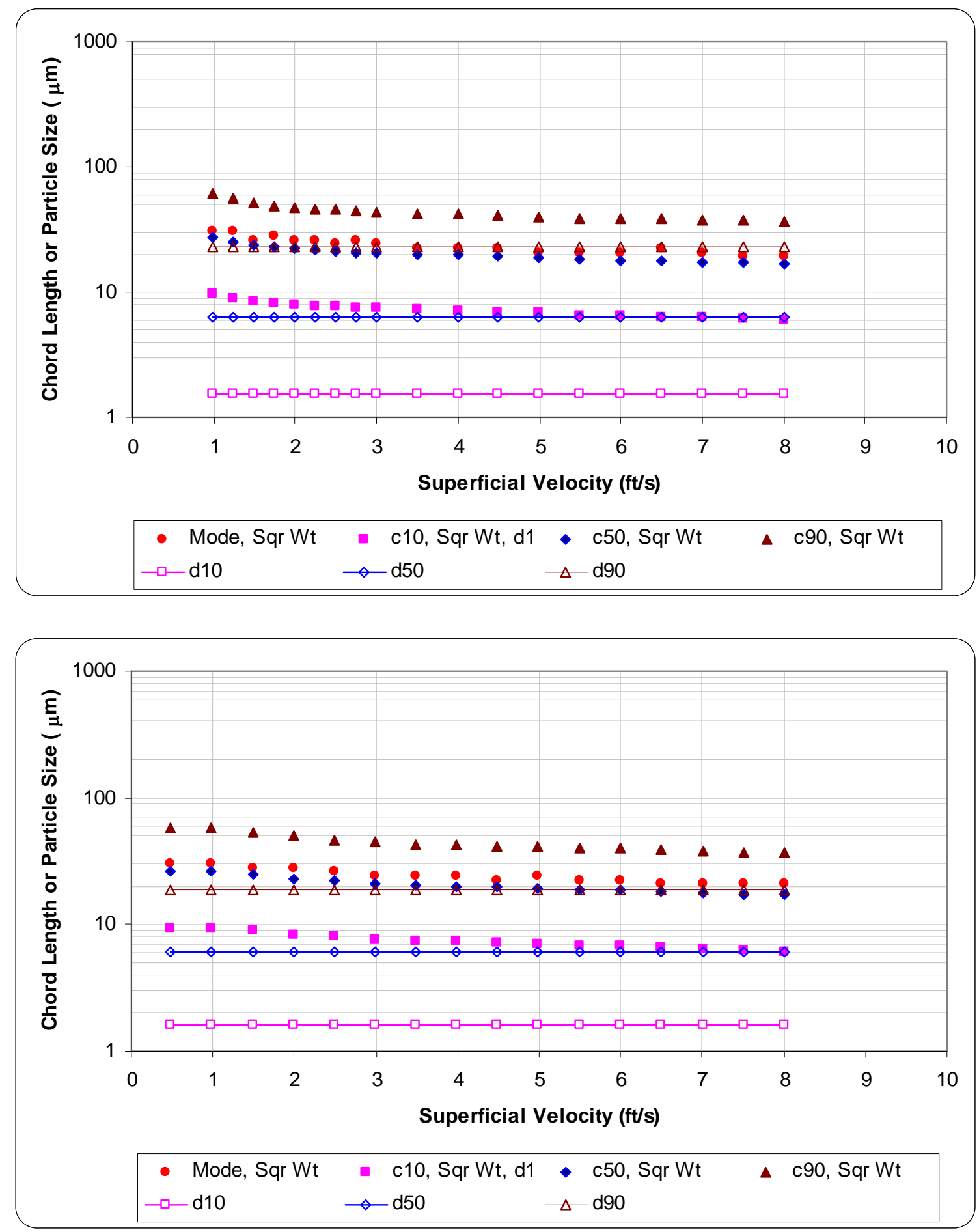


\section{B.2 Lasentec Results for HLL (top) HLM (middle) HLH (bottom) Systems; For Information Only}
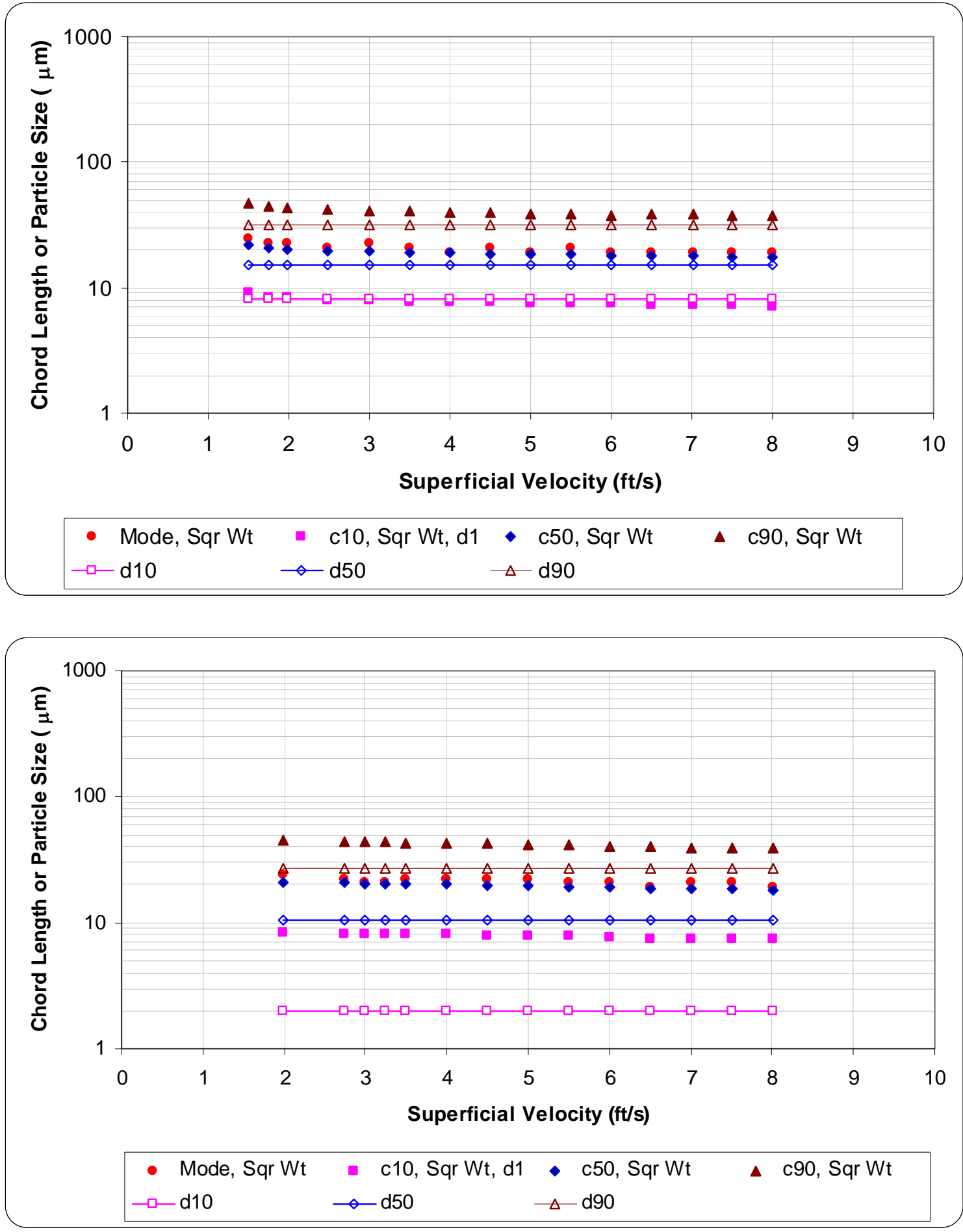


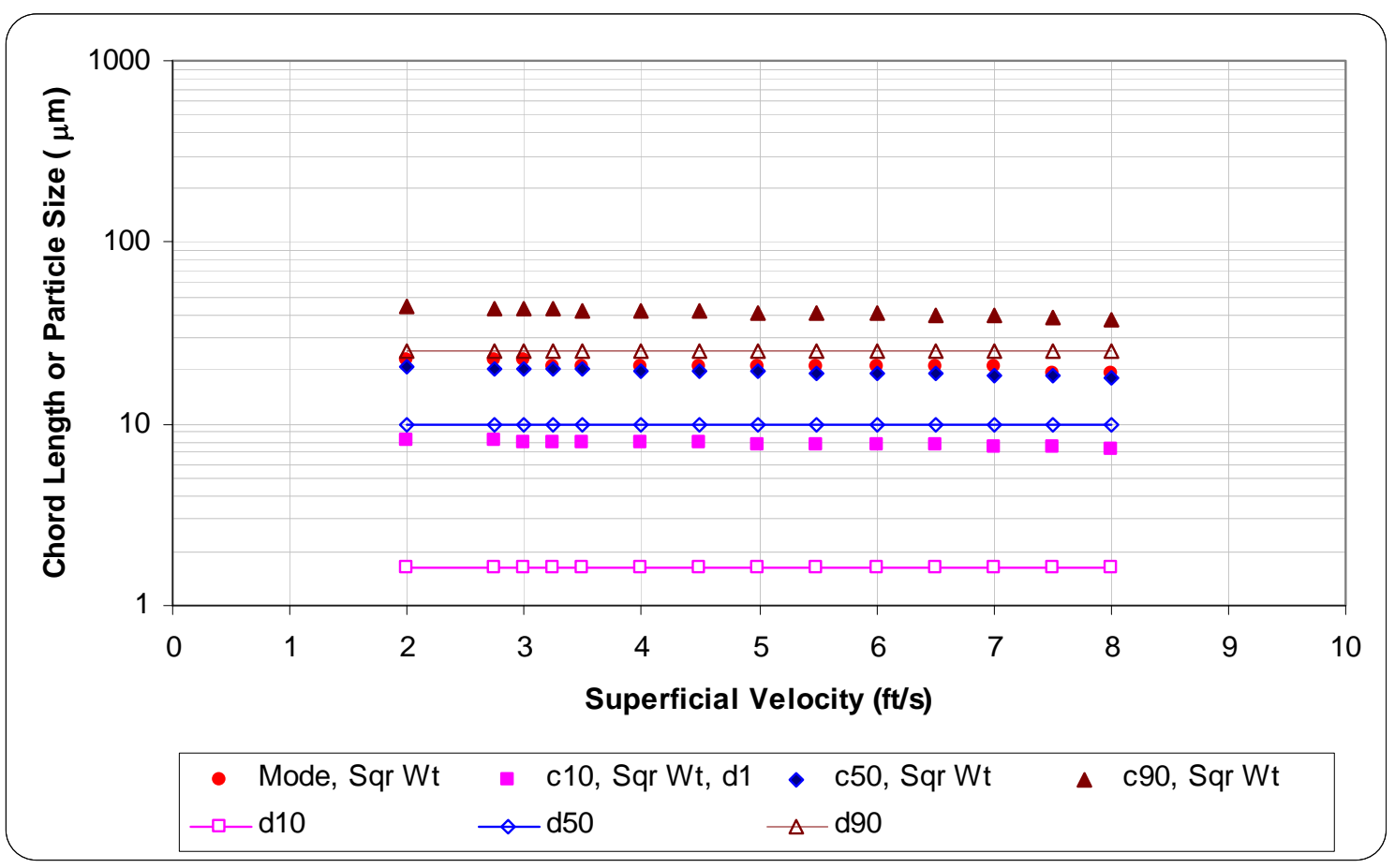

\section{B.3 Lasentec Results for MML (top) MMM (middle) MMH (bottom) Systems; For Information Only}

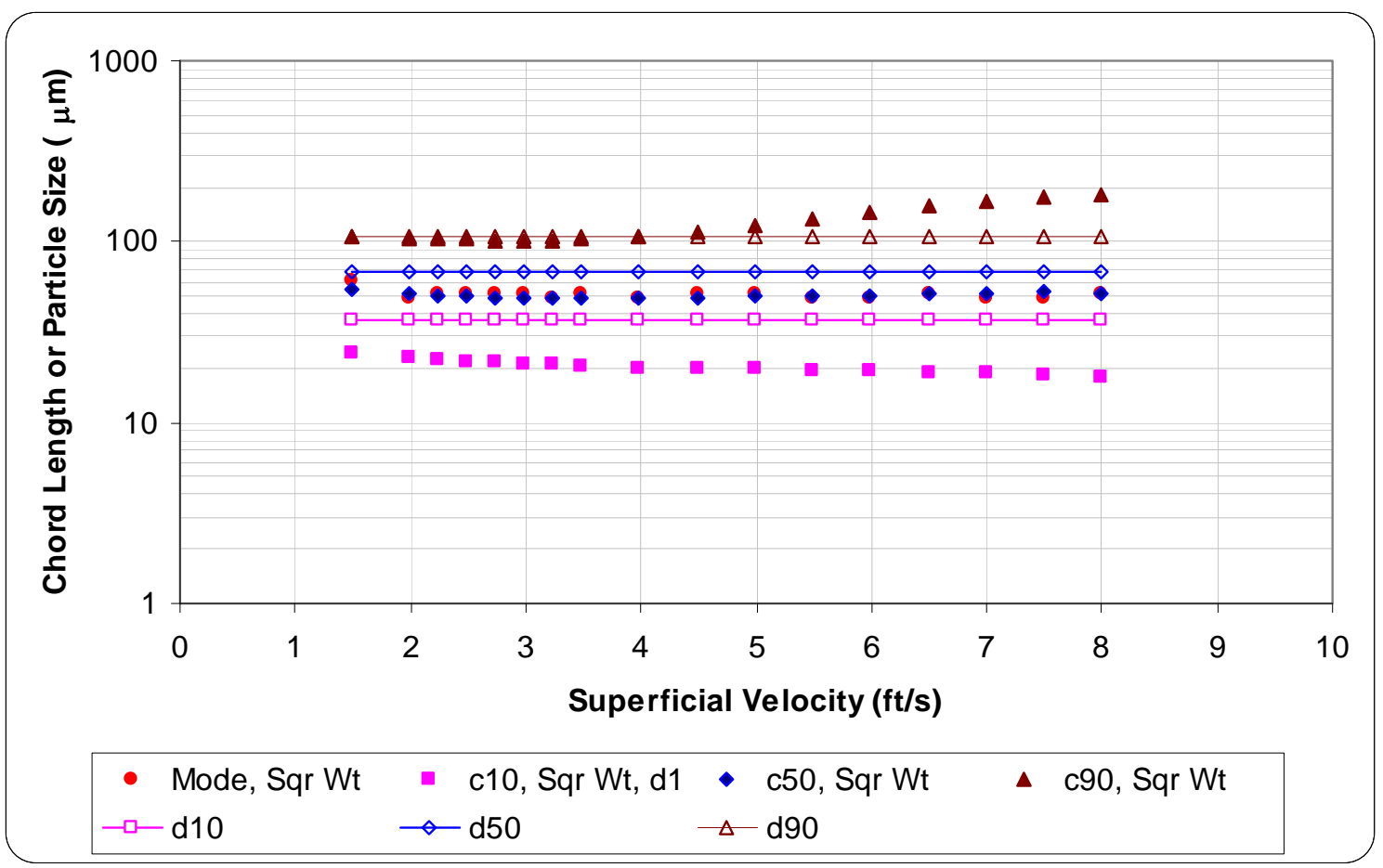



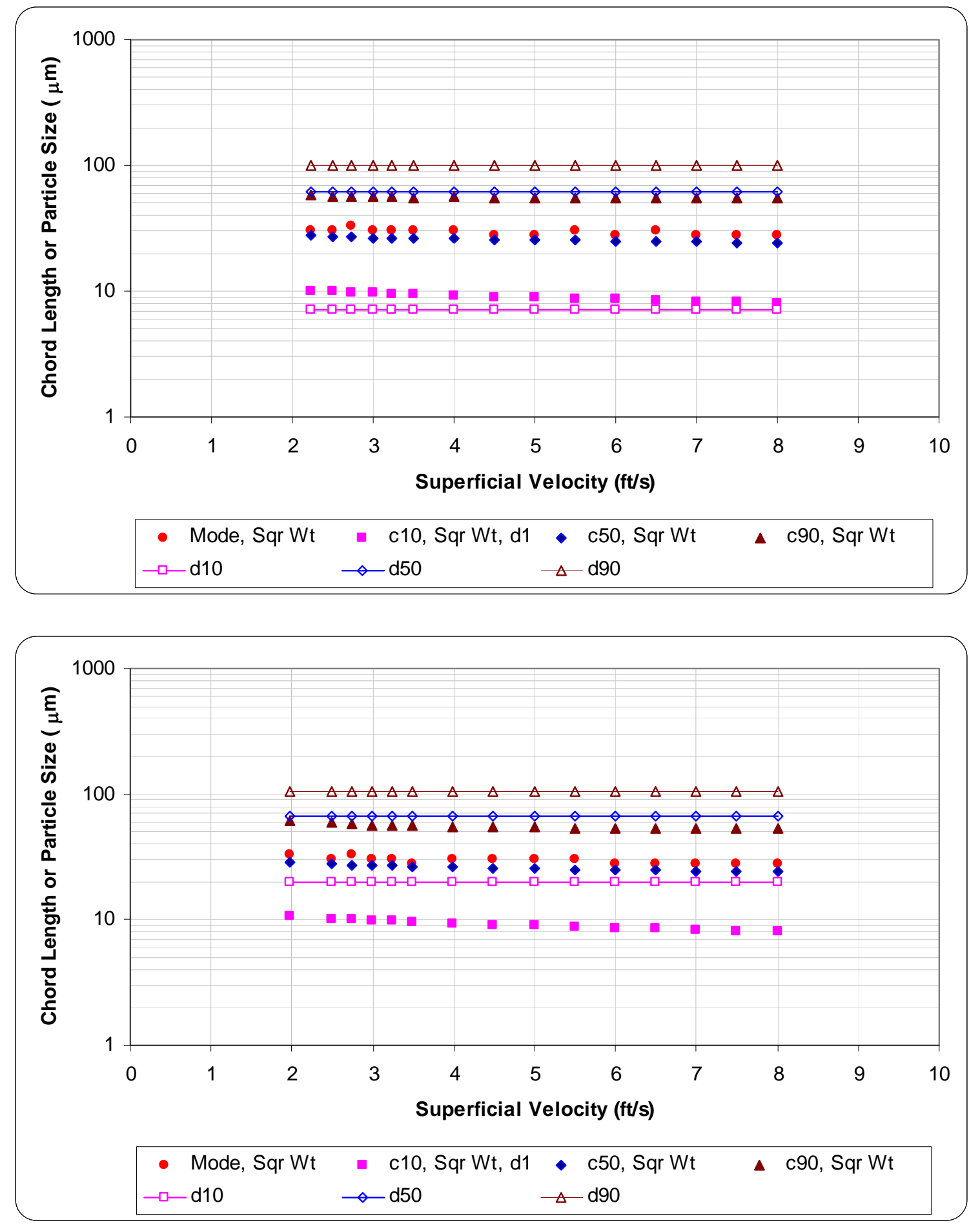


\section{B.4 Lasentec Results for LHL (top) LHM (middle) LHH (bottom) Systems; For Information Only}
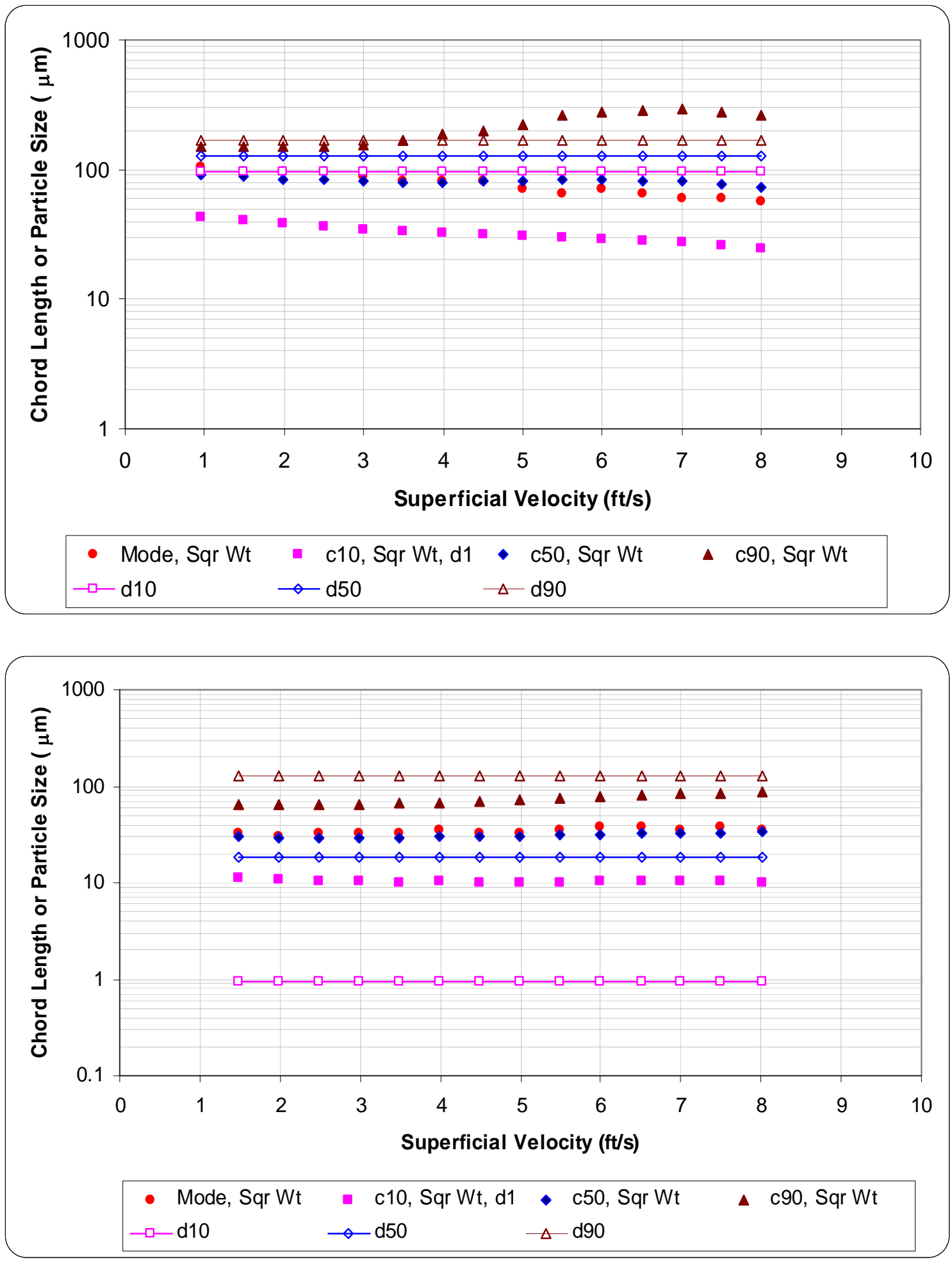


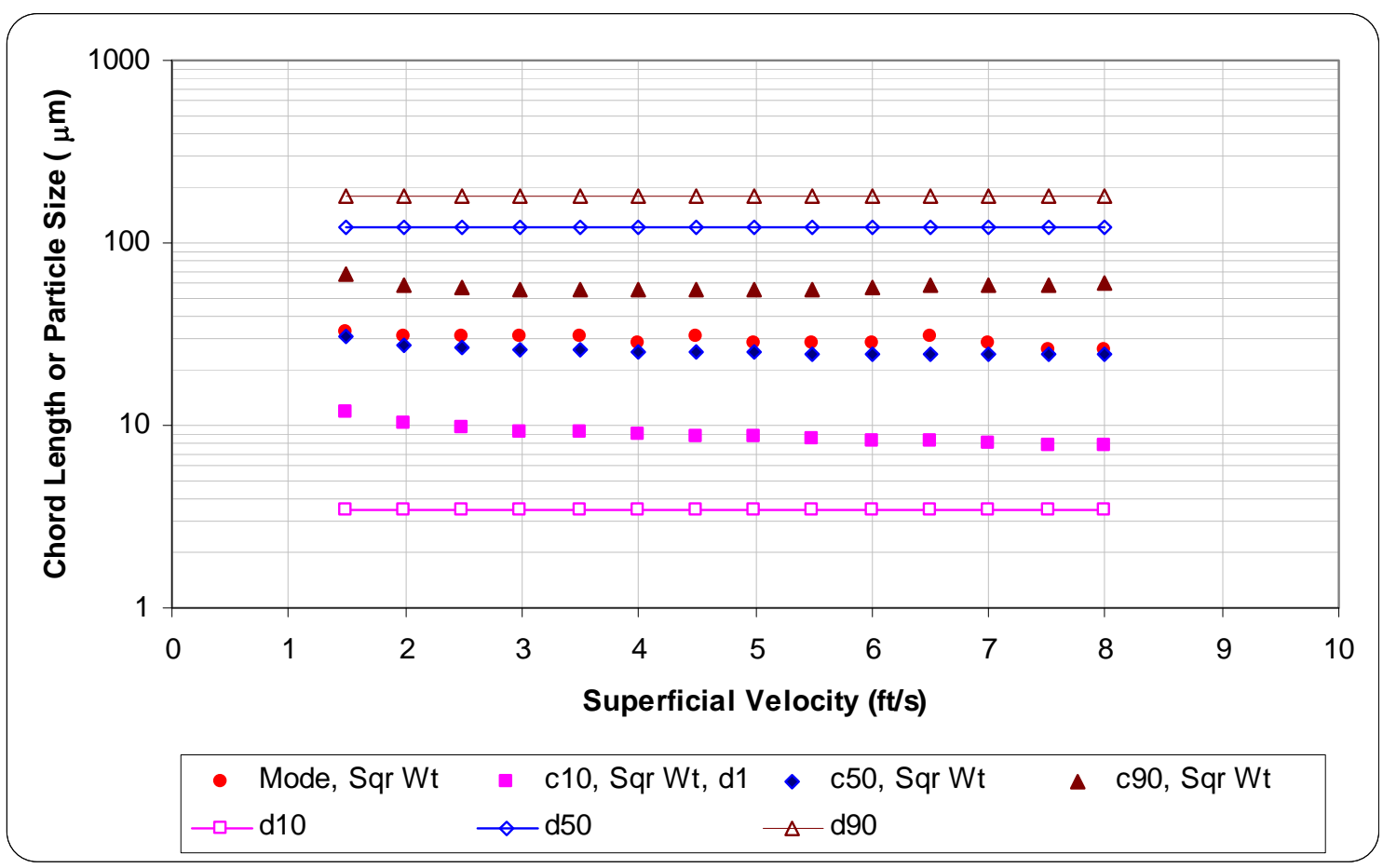

\section{B.5 Lasentec Results for HHL (top) HHM (middle) HHH (bottom) Systems; For Information Only}

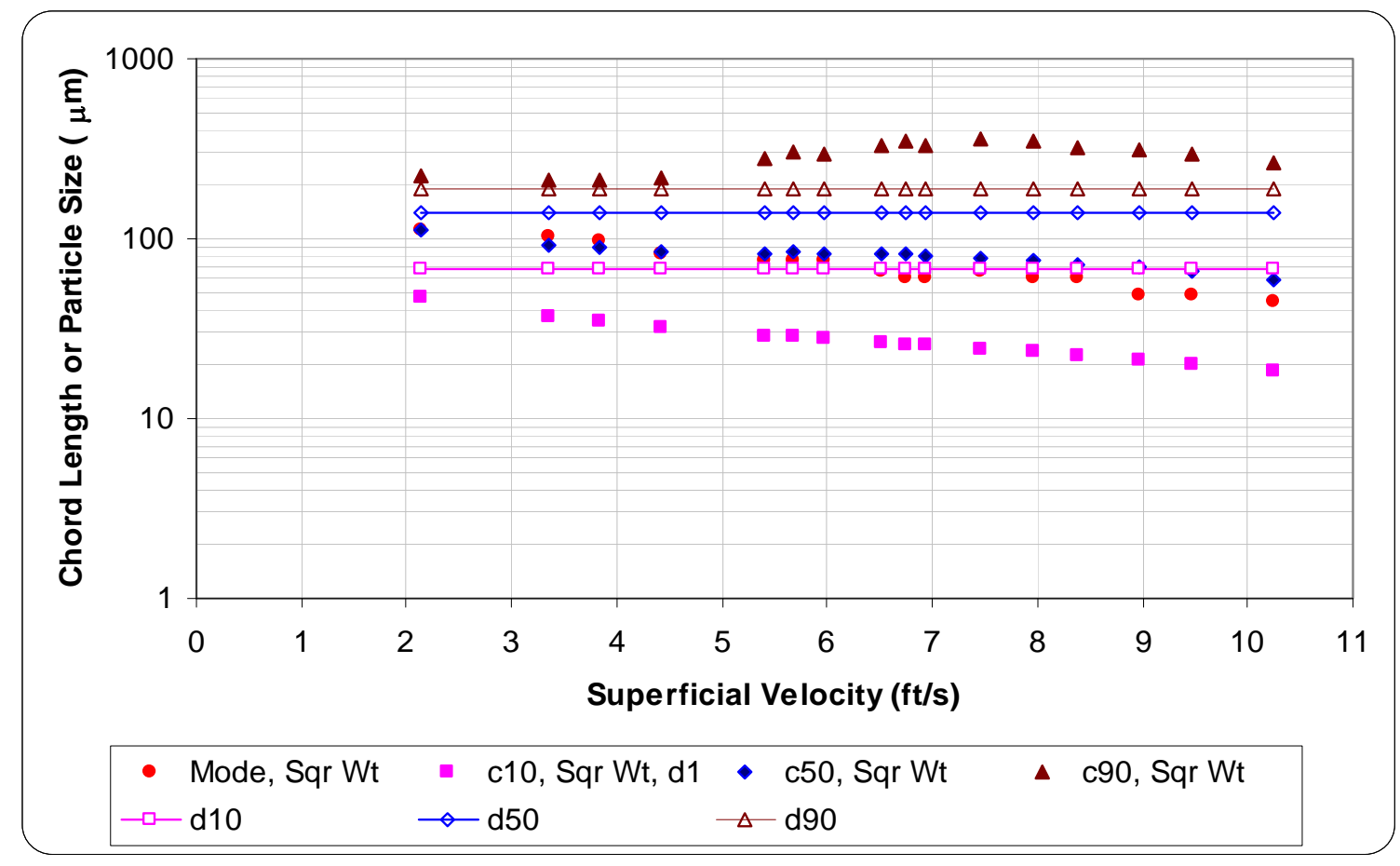



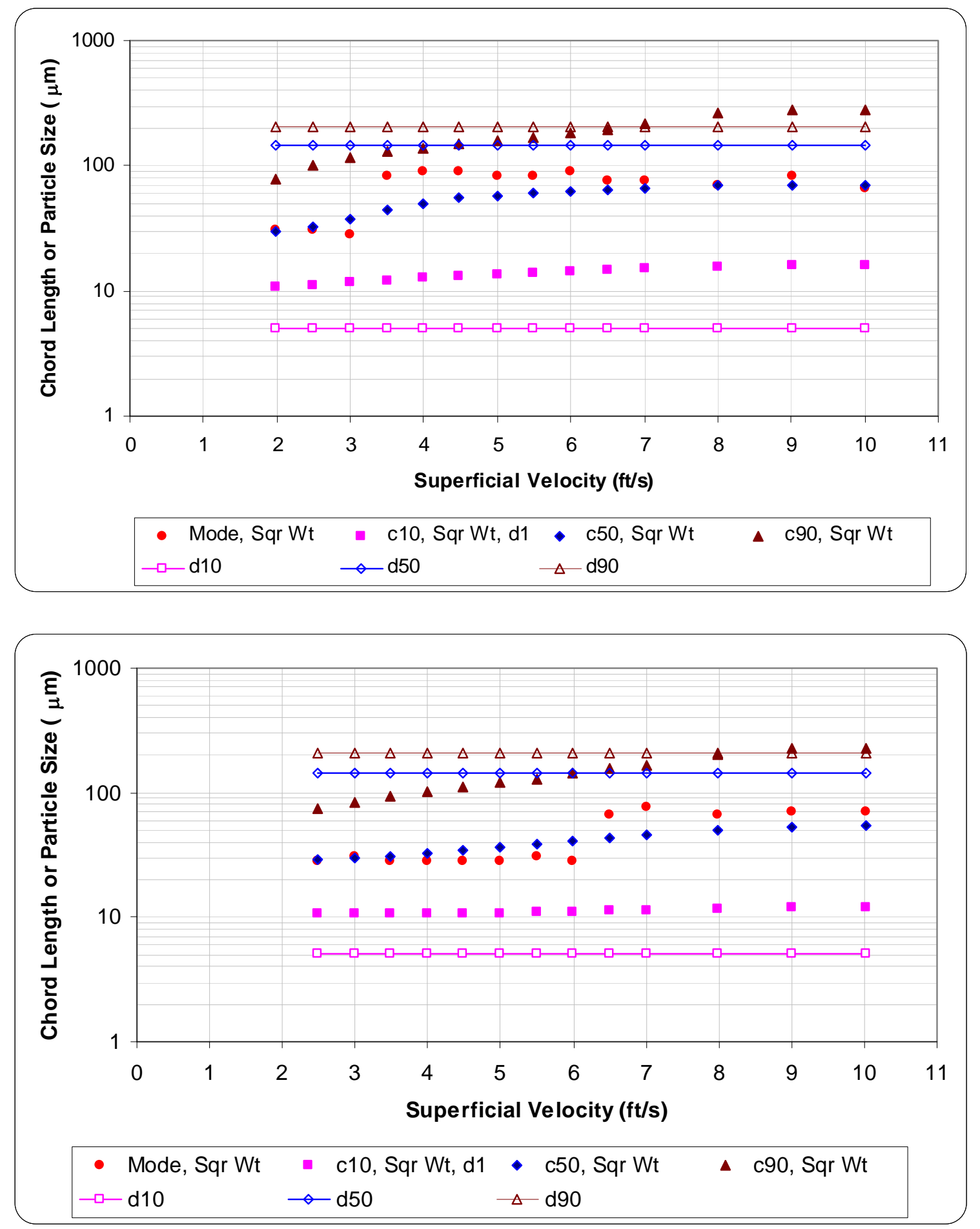
Appendix C

\section{Pressure Drop Versus Velocity for Water}





\section{Appendix C}

\section{Pressure Drop Versus Velocity for Water}

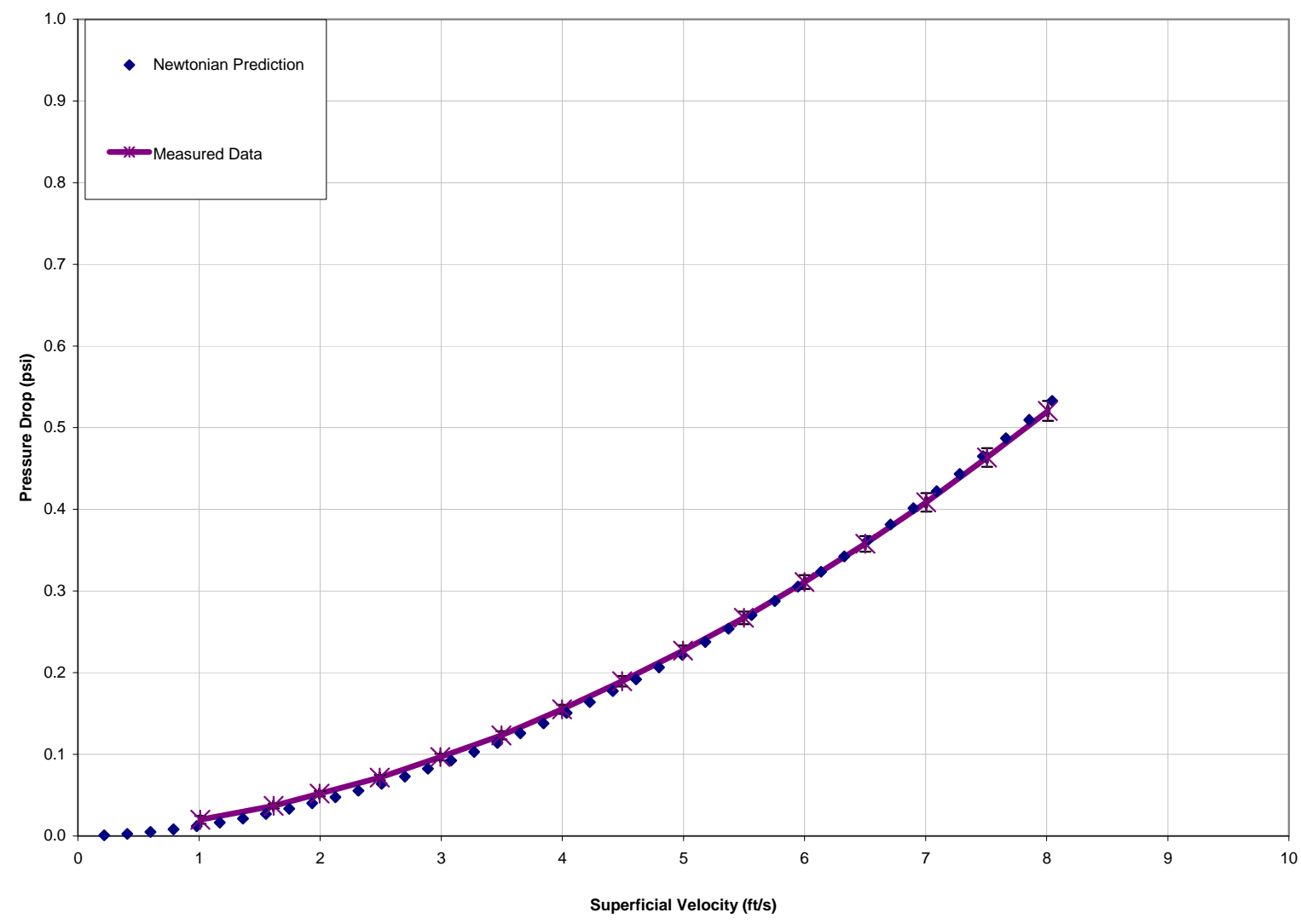



PNNL-17639

WTP-RPT-175 Rev. 0

\section{Distribution}

No. of

Copies

2 Bechtel National, Inc.

Darin Ramsey (2)

H4-02

10 Pacific Northwest National Laboratory

A. P. Poloski

H. E. Adkins

A. M. Casella

RE Hohimer

D. E. Kurath

M. J. Minette

J. M. Tingey

J. J. Toth

S. T. Yokuda

Project Files

Information Release (pdf)
P7-25

K7-15

P7-25

K7-50

K3-52

P7-25

P7-25

P7-22

K7-15

K3-52
No. of

Copies

Distr.1 